SAND82-7052

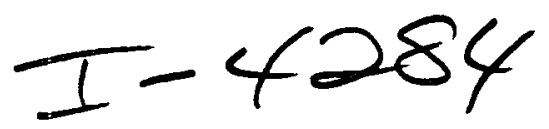

Unlimited Release

UC-66c

\title{
is \\ Geothermal-Well Completions: \\ A Survey and Technical Evaluation \\ of Existing Equipment and Needs
}

James E. Nicholson

Foster-Miller, Inc.

350 Second Avenue, Waltham, MA 02254

Robert E. Snyder

Completion Technology Corp.

4200 Westheimer Road, Houston, TX 77027

Prepared by Sandia National Laboratories Albuquerque, New Mexico 87185 and Livermore, California 94550 for the United States Department of Energy under Contract DE-AC04-76DP00789

Printed July 1982 


\section{DISCLAIMER}

This report was prepared as an account of work sponsored by an agency of the United States Government. Neither the United States Government nor any agency Thereof, nor any of their employees, makes any warranty, express or implied, or assumes any legal liability or responsibility for the accuracy, completeness, or usefulness of any information, apparatus, product, or process disclosed, or represents that its use would not infringe privately owned rights. Reference herein to any specific commercial product, process, or service by trade name, trademark, manufacturer, or otherwise does not necessarily constitute or imply its endorsement, recommendation, or favoring by the United States Government or any agency thereof. The views and opinions of authors expressed herein do not necessarily state or reflect those of the United States Government or any agency thereof. 


\section{DISCLAIMER}

Portions of this document may be illegible in electronic image products. Images are produced from the best available original document. 
Issued by Sandia National Laboratories, operated for the United States Department of Energy by Sandia Corporation.

NOTICE: This report was prepared as an account of work sponsored by an agency of the United States Government. Neither the United States Government nor any agency thereof, nor any of their employees, nor any of their contractors, subcontractors, or their employees, makes any warranty, express or implied, or assumes any legal liability or responsibility for the accuracy, completeness, or usefulness of any information, apparatus, product, or process disclosed, or represents that its use would not infringe privately owned rights. Reference herein to any specific commercial product, process, or service by trade name, trademark, manufacturer, or otherwise, does not necessarily constitute or imply its endorsement, recommendation, or favoring by the United States Government, any agency thereof or any of their contractors or subcontractors. The views and opinions expressed herein do not necessarily state or reflect those of the United States Government, any agency thereof or any of their contractors or subcontractors.

Printed in the United States of America Available from

National Technical Information Service

U.S. Department of Commerce

5285 Port Royal Road

Springfield, VA 22161

NTIS price codes

Printed copy: A02

Microfiche copy: A01 
SAND- $-82-7052$

DE82 018543

SAND 8 2- 7052

GEOTHERMAL-WELL COMPLETIONS :

A SURVEY AND TECHNICAL

EVALUATION OF EXISTING

EQUIPMENT AND NEEDS

James E. Nicholson

Foster-Miller, Inc.

350 Second Avenue

waltham, MA 02254

Robert E. Snyder

Completion Technology Corp.

4200 Westheimer Road

Houston, TX 77027

\section{ABSTRACT}

The geothermal environment and associated well completion problems are reviewed. Existing well completion equipment is surveyed and limitations are identified. A technical evaluation of selected completion equipment is presented. The technical evaluation concentrates on well cementing equipment and identifies potential failure mechanisms which limit the effectiveness of these tools. Equipment employed in sand control, perforating, and corrosion control are identified as potential subjects for future technical evaluation. 
This report has been divided into two major sections: a Survey of Existing Equipment and Needs (Section 2), and a Technical Evaluation of Casing Centralizers, Cementing Plugs, slotted Liner Washing and Casing Corrosion Due to Oxygen in Water Mist Air Drilling (Section 3). This work has been the combined effort of Foster-Miller Associates, Inc. (FMA), Waltham, MA, and Completion Technology Company (CTC), Houston, TX. FMA was primarily responsible for the technical evaluation of completion equipment, while CTC provided the survey of existing equipment and needs.

Contributions to this report were supplied by a number of individuals. At CTC, the major input was provided by Robert Snyder, with support from George suman and Edward T. Wood. Principal contributors at FMA were James Nicholson and David Dunfee with contributions from Khushroo Captain and Brian Doherty. Technical assistance was provided by Edwin Lee, Ron Lundin, and Patrick Ferolito.

This work was performed under Sandia Contract 49-8367. C. Carson of the Geothermal Technology Development Division was the Technical Contract Monitor. 
Section

Page

EXECUTIVE SUMMARY

ES-1

1 .

INTRODUCTION

$1-1$

1.1 Geothermal Environments

$1-1$

1.1.1 Dry Steam Wells

$1-2$

1.1.2 Hot Water Wells

$1-8$

2 .

SURVEY OF EXISTING EQUIPMENT AND NEEDS

$2-1$

2.1 Cementing Equipment Limitations

$2-1$

2.1.1 Introduction

$2-1$

2.1.2 Potential Failure Modes

$2-3$

2.1.3 Wiper Plugs

$2-4$

2.1.4 Spring Steel (Centralizer) Performance

$2-6$

2.1.5 Stage Collars

$2-10$

2. 2 Sand Control Problems

$2-14$

2.2.1 Implied Problems

$2-14$

2.2.2 Discussion

$2-14$

2.2.3 Identifying Problem zones

$2-16$

2.2.4 Limitations of Chemical Methods

$2-17$

2.2.5 Proposed Gravel Packing Concept

$2-18$

2.2.6 Completion Fluid/Gravel Pack Material

$2-21$

2.2.7 Recommendations

$2-22$

2.3 Effective Inhibitor Placement

$2-23$

2.3.1 Implied Problem

$2-23$

2.3.2 Discussion

$2-24$

2.3.3 Recommendations

$2-31$ 
$\underline{\text { Section }}$

$\underline{\text { Page }}$

2.4 Slotted Liner Limitations - Zonal Isolation

2.4.1 Implied Problem No. 1: Lack of Effective Zonal Isolation

2.4.2 Discussion

2.4.3 Recommendations

2.5 Slotted Liner Limitations - Liner Washing

2.5.1 Implied Problem No. 2: Limitations of Liner Washing Methods

2.5.2 Discussion

2.5.3 Mud Removal Mechanisms

2.5.4 Washing Concepts

2.5.5 Recommendations

2.6 Geothermal Well Perforator Limitations

$2-51$

2.6.1 Implied Problem

$2-5 I$

2.6.2 Discussion

$2-51$

2.6.3 Explosives Used in Modern Well Perforators

$2-53$

2.6.4 Components of Well Perforators

2.6.5 Well Perforating Systems

2.6.6 Recommendations for Evaluation of Wel1

Perforators at Elevated Temperatures

3.

$$
\text { TECHNICAL EVALUATIONS OF CASING CENTRALIZERS, }
$$
CEMENTING PLUGS AND SLOTTER LINER WASHING

3.1 Centralizer Bow Frictional Heating and Wear 
Section

$\underline{\text { Page }}$

3.1 .1 Introduction

3.1.2 Casing Centralizers

3-3

3.1.3 Geothermal Well Completions 3-8

3.1.4 Casing Centralizers Run in Geothermal Wells 3-15

3.1.5 Thermal Analysis of the Centralizer Bow 3-19

3.1.6 Wear Analysis of the Centralizer Bow 3-25

3.1.7 Conclusions 3-40

3.1.8 Recommendations 3-41

3.2 Geothermal Well Cementing Plug Failure Due to Frictional Heating and wear

3.2.1 Introduction

3.2.2 Cement Wiper Plugs

3.2.3 Cement Plug Pressure Loading on Casing Side Walls

3.2.4 Thermal Analysis of Wiper Ring

3.2.5 Cementing Plug Wear

3.2.6 Cement Plug Effectiveness 3-61

3.2.7 Conclusions 3-62

3.2.8 Recommendations 3-62

3.3 Technical Evaluation of Slotted Liner Washing 3-62

3.3.1 Introduction 3-62

3.3.2 Description of Slotted Liners 3-64

3.3.3 Fluid Dynamics of Flow Between a slotted Liner and an Open Hole 
TABLE OF CONTENTS (Continued)

Section

Page

3.3.4 Experimental Evaluation of Slotted Liner Washing Methods

$3-72$

3.3.5 Conclusions

$3-81$

3.3.6 Recommendations

4.

REFERENCES

$4-1$

4.1 References for Subsection 2.1

$4-1$

4.2 References for Subsection 2.2

$4-1$

4.3

References and Industry Contacts for Subsection 2.3

$4-2$

4.4 References and Industry Contacts for Subsection 2.4

$4-3$

4.5 References for Subsection 2.6

$4-4$

4.6 References for Subsection 3.1

$4-4$

4.7 References for Subsection 3.2

$4-6$

4.8

References for Subsection 3.3

$4-6$ 
Figure

$\underline{\text { Page }}$

1-1 BOP stack for air arilling

$1-3$

1-2 Casing wear by arill pipe

$1-4$

1-3 Modern Geysers' casing program

$1-5$

1-4 Casing problems that can be more severe in geothermal wells due to high temperatures and large diameter strings commonly used

$1-6$

1-5 Weld-type casing head with top flange and side ports and dual master valves

$1-7$

1-6 Model of a high-temperature hot-water geothermal system

$1-9$

$1-7$

Typical downhole problems in hot water wells

$1-10$

$1-8$

Perforation tunnel through pipe and cement

$1-11$

$1-$

Schematic of cemented and perforated casing

$1-12$

1-10 Typical Imperial Valley completions for two well depths

$1-14$

1-Il Hot water producing well with expansion spool on wellhead

$1-12$

Stress behavior in solidly cemented casings

Considerations for cementing wiper plug functional analysis

2-2 Schematic representation of centralizer bow spring moving through casing to open hole

2-3 Cross-section of centralizer bow on casing inside diameter

2-4 Schematic of stage tool operation

2-5 Schematic illustration of two types of stage tools - rotating sleeve and vertical action sleeve 
Increase in casing radius with pressure and temperature for several types and sizes of casing

2-7 Completion which gravel packs only the sandproducing zones

2-8 Detail for selective gravel and cement placement behind liner in geothermal well

Inhibitor injection in flowing well

Pumped production well

2-11 Scheme for inhibitor placement in a pumped well

2-12 Casing running sequence with external tubing string

2-13 Concept of zonal isolation between sand intervals in an Imperial Valley production of injection well

2-14 Concept of circulating cement slurry through a thick shale interval between slotted liner sections

2-15 Example Saraband log section from East Mesa well showing thick mud cake over porous sand intervals

2-16 Schematic of wash fluid removing mud behind slotted Iiner

2-17 Wash tool packers can be spaced in a variety of configurations (source Cavins catalog 8081)

2-18 Wash tool operations including bypass bore, treating port, and top circulating port (source Cavins catalog 8081)

2-19 Example commercial wash tools 
Figure

Page

2-20 Perforation wash tool and concept of open cavity being formed behind perforated casing (source $B \& W$, Inc., Composite Catalog 1980-81)

2-21 Courtesy of NL MCCullough

2-22 Temperature response to a thermocouple attached to a 2-1/2-in. HMX perforating gun during temperature test

2-23 Detonating stages of a typical shaped charge (courtesy of Dresser Atlas)

$2-60$

2-24 Hollow steel carriers and expendable guns (courtesy of Welex)

2-25 Schematic diagram of gun perforator testing setup - Berea sandstone core target (courtesy of American Petroleum Institute)

3-1 Casing buckling of unsupported pipe with heating (after snyder)

3-2 Example of spring-bow centralizer contained by stop collar so that device is pulled into hole

3-3 Tool-joint and drillstring contact forces in dog-legs versus axial tension - 4.5 in. drillpipe

$B \& W$ basic centralizer selection guide

3-5 Casing plan for representative East Mesa Well (Imperial Valley) model

Modern Geysers casing program 


\section{LIST OF ILLUSTRATIONS (Continued)}

Figure

Page

3-8 How decentralization affects velocity on the narrow side of the annulus in relation to overall rate of flow for one set of fluid and hole conditions (from Suman)

3-9 Forces acting to displace, and resist displacement of a bypassed vertical mud column during primary cementing (from Suman)

3-10 Centralizer bow thermal model

3-11 Centralizer bow: transient temperature

history at bow center

3-12 Centralizer bow spring model - no wear

3-13 Centralizer bow spring model - worn through

$3-34$

3-14 Centralizer bow spring model - 50 percent worn

3-15 Centralizer bow deflection

3-16 Typical primary cementing job

3-17 Type of curve that can be designed for individual conditions to determine additional cement, to assure coverage of the narrow side of eccentric annulus

3-18 Top and bottom wiper plugs

3-19 7-in. top cementing plug recommended for 17 to $38 \mathrm{lb} / \mathrm{ft}$ casing

3-20 Casing internal diameter and cement plug external diameter versus casing size and weight

3-21 Cement plug interference fit with casing internal diameter for various casing sizes and manufacturer recommended cementing plugs 
Figure

Page

3-22 Schematic representation of cementing plug interference fit into casing

3-23 Plastic tube-strain-gaged and pressurized to measure internal pressure versus strain

3-24 Internal pressure versus strain 7-in. cementing plug

3-25 Temperature profiles within static and producing high-temperature wells in the

Imperial Valley

3-26 Master curve for coefficient of friction of nitrile rubber

3-27 Abradability $-\gamma-\mathrm{mm}^{3} / \mathrm{kg} \cdot \mathrm{m}$

3-28 Master curves obtained experimentally for

the abrasion of three unfilled rubbers

3-29 Typical slot pattern in 7-in. liner - 26-1b NYU and K55 casing

3-30 Flow pattern from slot jet impinging on open hole wall

3-31 Jet flow

3-32 Stagnation in plane flow

3-33 Velocity distribution in the laminar boundary layer on a flat wall in the vicinity of the stagnation point

3-34 Experimental test apparatus

3-35 Hole pattern in 4.5-in. pipe for slotted liner washing tests

3-36 Jet dynamic pressure distribution 


\section{LIST OF ILLUSTRATIONS (Continued)}

Eigure

$\underline{\text { Page }}$

3-38 Testing of soft plaster-mud samples at slot velocity of $1.7 \mathrm{ft} / \mathrm{sec}, 5-\mathrm{min}$ sample exposure

3-39 Testing of medium-hard plaster-mud samples at slot velocity of $1.7 \mathrm{ft} / \mathrm{sec}, 5-\mathrm{min}$ sample exposure

3-40 Testing of firm plaster-mud samples at slot velocity of $1.7 \mathrm{ft} / \mathrm{sec}, 15-\mathrm{min}$ sample exposure 


\section{IIST OF TABLES}

Table

Page

1-1 Water analysis of a Salton Sea geothermal well

2-1 Variations in temperature ratings by explosive type

$2-55$

3-1 Digest of cementing equipment and mechanical aids

3-2 Temperature rise at midpoint of centralizer bow

3-3 Wear constant $k$ of various sliding 3-26 combinations

3-4 Abrasive wear constants $k$

3-5 Wear constant from drill pipe tripping wear on casing

3-6 Wear constant from wireline wear on casing

3-7 Summary of wear constant data for drill pipe and wireline wear on casing

3-8 Probable centralizer bow wear 800-1b side force, 5000-ft travel

3-9 Common materials for cementing plugs

3-10 Interference between top cementing plug and 7 -in. casing

3-11 Transient time history of rubber slab 3-55

3-12 Nominal slotted liner conditions 3-65

3-13 Velocity distribution at $x=0.1$ in. $\left(\mathrm{U}_{\text {jet }}=1 \mathrm{ft} / \mathrm{sec}\right)$

3-14 Average shear stress between slotted liner and wall ( 7 and 8.75 in., respectively) 


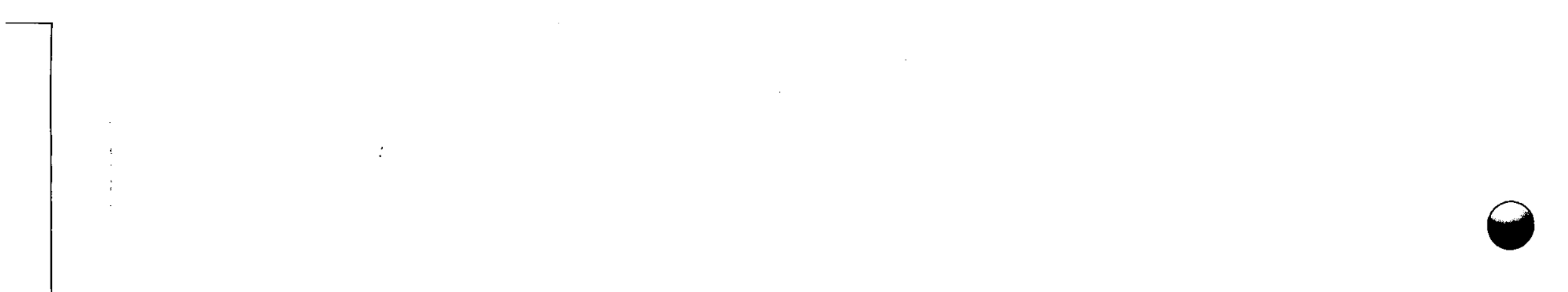


Many problems exist with geothermal completions, primarily because the environmental temperature exceeds the operational limits of existing well drilling equipment, and the quantity of geothermal wells to be drilled (70 in 1980) does not justify new product development for these hostile environments. Well completion companies are forced to work with existing equipment due to the lack of equipment and techniques specifically designed for the geothermal environment. In addition, inquiries by these investigations into potential equipment problems have not yielded great amounts of failure information, primarily because the equipment design details are proprietary, and in part because the geothermal marketplace is small in contrast to the vastly larger oil well drilling market (approximately 70,000 wells drilled annually).

This report has been divided into two major parts. The geothermal environment and associated completion problems have been introduced in section 1. Section 2 is a survey of existing equipment and needs and section 3 is a technical evaluation of selected completion devices which have been identified in section 2 as elements within a major problem area.

Beginning with section 2, the following major areas were identified as being potential areas of equipment or methodology improvement:
a. Cementing equipment
b. Sand control
c. Corrosion prevention
d. Slotted liner limitations
e. Well perforator limitations.

The major focus has been on cementing equipment due to the fact that cementing problems, including lost circulation, account for one of the largest sources of problems encountered in geothermal completions. Limitations of cement and the cementing equipment at elevated temperatures may lead to incomplete cementing, which has been identified as a major factor in abandonment of several Baca wells.

Sand control problems, especially in the Imperial Valley, California, are a potential source of well equipment erosion and damage to pumping and surface treatment equipment. Sand control at elevated temperatures is difficult and involves complex combinations of wire screens and gravel packing with gravel of a precise size range. Problems and potential solutions are discussed. 
Corrosion during aerated water drilling at Baca has been measured to be $24 \mathrm{lb} / \mathrm{ft} 2 /$ year. By increasing the $\mathrm{pH}$ of the drilling fluids to 10.5 to 11.0 , corrosion rates have been reduced to $2 \mathrm{lb} / \mathrm{ft} 2 /$ year. Effective placement of corrosion inhibitor is a necessary step in controlling corrosion. The problem and potential solutions are discussed.

slotted liners are used in the Geysers and Roosevelt Hot springs, where the formations allow completions with an open hole. A continuous slotted liner assumes that the open hole is capable of producing uniformly over the entire length. Temperature surveys have shown that the producing zones are intermittent and it may be advisable to seal off certain nonproducing zones. This leads to the problem of how to seal off the slotted liner selectively. This problem is described and potential solutions are suggested.

When open holes are drilled with the drilling mud and the slotted liners are installed, it is necessary to wash the drilling mud from the open hole and liner. At present, in order to wash the hole, water is circulated downhole, out through 3 to 5 ft of slotted liner, and back up the center of the slotted liner. The technique is described as well as the problems attendant on the method.

In geothermal areas where formations cannot be completed in an open hole, the hole is cased and cemented. Geothermal fluid is collected by perforating the casing and cement with shaped charges. The major difficulty with this technique is that the explosives are affected by elevated pressures and temperatures. The highest temperature explosive, HNS, will withstand 5000F for several hours, but the explosive is expensive and does not perform as well as HMX or RDX. The explosives and their limitations are discussed as well as the need for improvements.

Section 3 is a technical assessment of three problems identified in Section 2. They are:

a. Centralizer bow frictional heating and wear

b. Cementing plug heating and wear

c. Slotted liner washing.

The centralizer bow is the primary means of keeping a casing central within a hole while the casing is cemented in place. Normally, geothermal wells are not straight, but deviate at rates on the order of $2 \mathrm{deg} / 100 \mathrm{ft}$. The weight of the casing and the deviated holes cause large sideloads to be exerted on the centralizers, thus making the centralizing job difficult. While the centralizer is pushed downhole, the contact area between the bow 
and the sidewall is heated by friction and worn due to abrasive wear. In many cases the frictional heating is sufficient to reduce the bow strength by annealing the metal. In addition, abrasive wear of the bow may reduce the cross-section of the centralizer such that the casing will shift substantially from center. A 25-percent shift in the centralized casing is sufficient to cause no cement flow on the narrow side at a pumping rate of $5 \mathrm{bbl} / \mathrm{min}$. The problem is described in detail and recommendations are made to reduce frictional heating and improve wear.

Cementing plugs are normally used to isolate the cement which is used to cement geothermal casing in place. The cementing plugs are molded from elastomers such as nitrile, which has a maximum recommended temperature of $300^{\circ} \mathrm{F}$. The ears of the cement plug are oversized so that they bend back while being pushed downhole. The frictional heating and thermal conductivity of the rubber is such that the material temperature rapidly rises to a temperature where it is quickly abraded away. In less than 1000 ft of wall contact with casing at elevated temperature, the cement plug will be reduced to a separator rather than a wall mud scraper, as suggested by the design. As a separator, the cementing plug will do little to prevent casing mud from mixing with the cement. The problem is described and discussed in detail.

Slotted liners are manufactured by saw cutting slots at several positions along a casing. The slots are typically spaced 6 in. apart at a density of 16 slots per ft. Special tools have been developed to wash the exterior of the slotted liner as well as the mud-cake-covered open hole into which the slotted liner is inserted. Water is injected between opposite facing cups located within the slotted liner out to the open hole and back into the liner above the cups. Typical pumping rates are 5 to $10 \mathrm{bbl} / \mathrm{min}$ to clean 3 to $5 \mathrm{ft}$ of slotted liner and open hole at a time.

For the pumping rates and cleaning geometries used, the typical fluid velocity out of the slot is 1.7 to $5.1 \mathrm{ft} / \mathrm{sec}$. The jet of fluid spreads minimally such that the cleaning action is localized to the area immediately adjacent to the slot.

Shear outside of this area due to the jet flow is considered to be inadequate to give effective cleaning. Experimental evidence of flow pressures and cleaning supports the analysis. The problem and methods are described. 
e 


\section{INTRODUCTION}

As geothermal energy utilization has advanced over the past few years, it has become apparent, as in any expanding technology, that further development work is required to improve the "tools of the trade." One of several areas in which advancement in the state of the art is required is well completion technology. As in drilling, completion equipment and procedures have grown from the technology base which exists in the oil patch. The basic problem is similar: that is, holes generally must be drilled from the surface into producing zones, and means must be provided to allow the produced fluid to flow to the surface. Hole diameters are generally somewhat larger, for a given depth, but use existing conventional bit, casing, and liner sizes.

The major difference lies in the much higher temperatures, the higher flow rate and the more corrosive and erosive environmental conditions found in a geothermal well. As a generalization, downhole equipment in an average (that is, 6000 to $8000 \mathrm{ft}$ ) geothermal well is subjected to environmental conditions which are found in very deep $(20,000$ to $24,000 \mathrm{ft})$ oil wells. Thus, the accelerated equipment failure rate experienced in very deep oil wells is found in significantly shallower geothermal wells.

Further, commercial oil equipment producers are extremely busy meeting the equipment needs for the 70,000 oil wells drilled each year in the United States, and are generally not yet attracted to the specific needs of the 70 geothermal wells drilled in 1980 in the United States. The few United States geothermal operators are therefore forced to improvise, or make do with existing oil and gas completion equipment. If the life, dependability, and acquisition and operating cost of geothermal completion equipment is improved, the commercial growth of geothermal energy use may be accelerated.

\subsection{Geothermal Environments}

Geothermal operations include superheated water and steam fields in the western United States. These developments are exemplified by unusual but not rare, dry steam-producing wells in The Geysers, California, and by hot brine-producing wells in California's Imperial Valley. Conditions broadly classified as "geothermal" are found in experimental wells in the "hot, dry rocks" program in New Mexico and in very high pressure, very deep hot water/methane environments in Texas and Louisiana. The latter two developments are out of the scope of this contract, although both will indirectly benefit from any resulting hot well technology innovations. 
Well completion equipment, including all casing and liner strings, wellheads, wing valves, gauges, controls, permanent downhole hangers, bridge plugs, packers, and cement, will be exposed to both high-temperature and corrosive and/or abrasive aqueous fluids. Typical conditions upon which studies were based are described in the following discussions.

\subsubsection{Dry Steam Wells}

In The Geysers, typical reservoir temperatures are $500^{\circ} \mathrm{F}$ in $500 \mathrm{lb} /$ in. 2 dry steam at 6000 - to $8000-\mathrm{ft}$ depths with cooling to about $370^{\circ} \mathrm{F}$ at $125 \mathrm{lb} / \mathrm{in} .2$ at the wellhead. This steam is dry, dusty and quite abrasive to surface equipment such as ells and tees. It is corrosive when it condenses and forms small wet volumes of $\mathrm{CO}_{2}$ and $\mathrm{H}_{2} \mathrm{~S}$ gases. Typical flow volumes are 150,000 to less than $400,000 \mathrm{lb} / \mathrm{hr}$ per well.

The Geysers' sieam fields are located in hard, fractured volcanic rocks that are difficult to drill from surface to total depth (TD). The area is mountainous and wells are drilled directionally from central locations, or pads, to desired bottom hole locations. This type of drilling is found in other mountainous geothermal areas in and around extinct volcano calderas, etc., for example, in the Baca field near Albuquerque, NM. So, developments desired for The Geysers would be widely applicable.

Permanent well equipment is greatly affected by rough drilling. Surface casing, wellheads and master valves suffer a great deal of abuse due to vibration, abrasive flow, and drill pipe wear. Figure 1-l shows how these three items are utilized even during drilling, to support a BOP stack that can weigh over 20 tons.

The Geysers' wells are partially mud drilled, through problem-flowing serpentine sections and shallow water flows. Systems are converted to air to drill into the fractured zone containing dry steam. As hydrostatic pressures of drilling muds would be thousands of pounds per square inch, such steam zones would be instantly plugged and quenched by mud or water.

Air drilling takes its toll on cemented casing as the hole environment prevents use of rubber drill pipe protectors. If hole deviation exceeds $1-1 / 2$ to $3 \mathrm{deg} / 100 \mathrm{ft}$ which is possible, the "doglegs" accentuate drill pipe casing wear as in Figure 1-2. oxygen entrained in the hot, steam-cut return stream creates highly corrosive/erosive conditions that rapidly deteriorate drill pipe. Exposed casing and liners would be similarly damaged. 


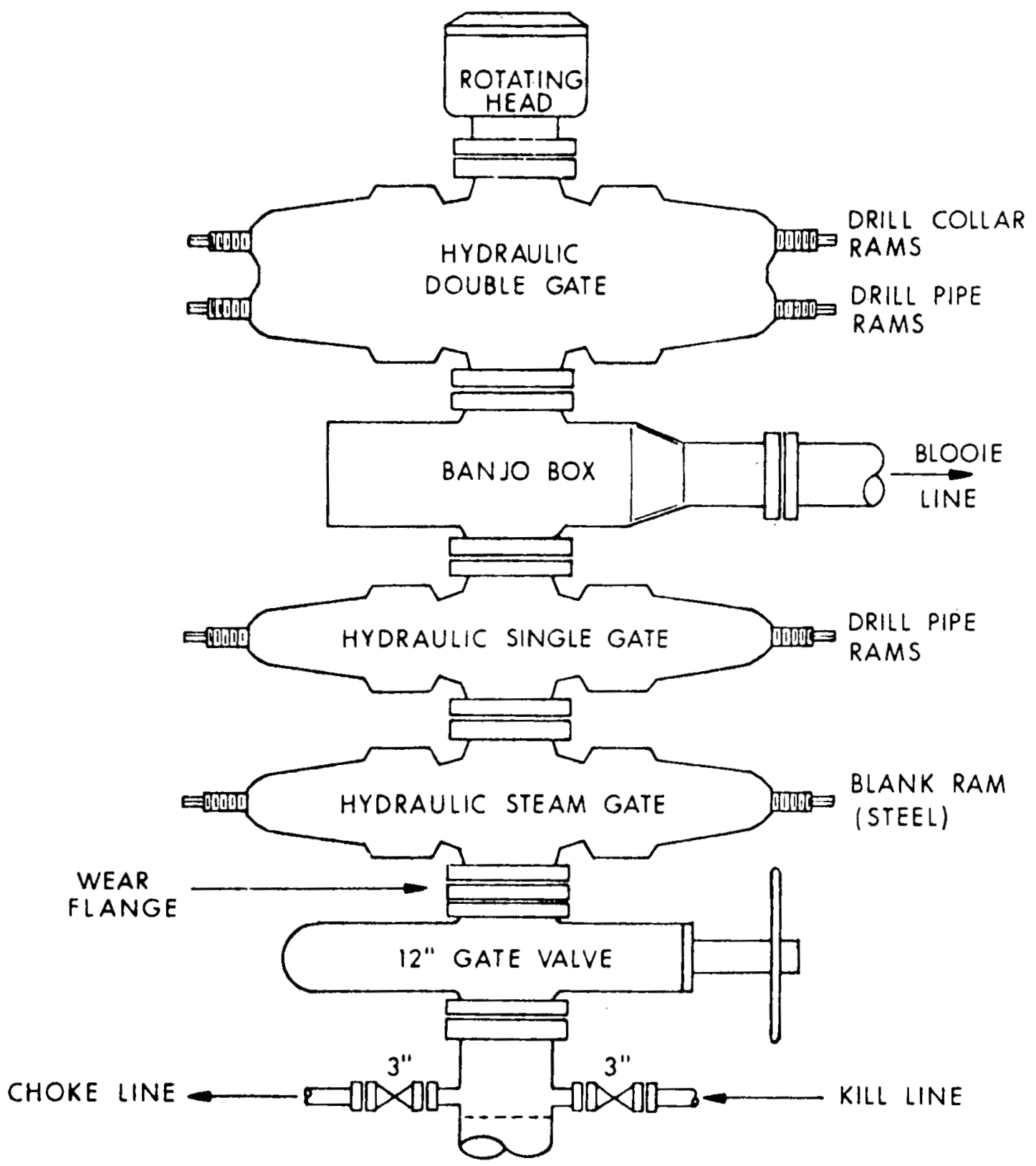

13-3/8" CASING HEAD

FIGURE 1-l. - BOP stack for air drilling. Banjo box diverts air and steam to muffler and pit. 


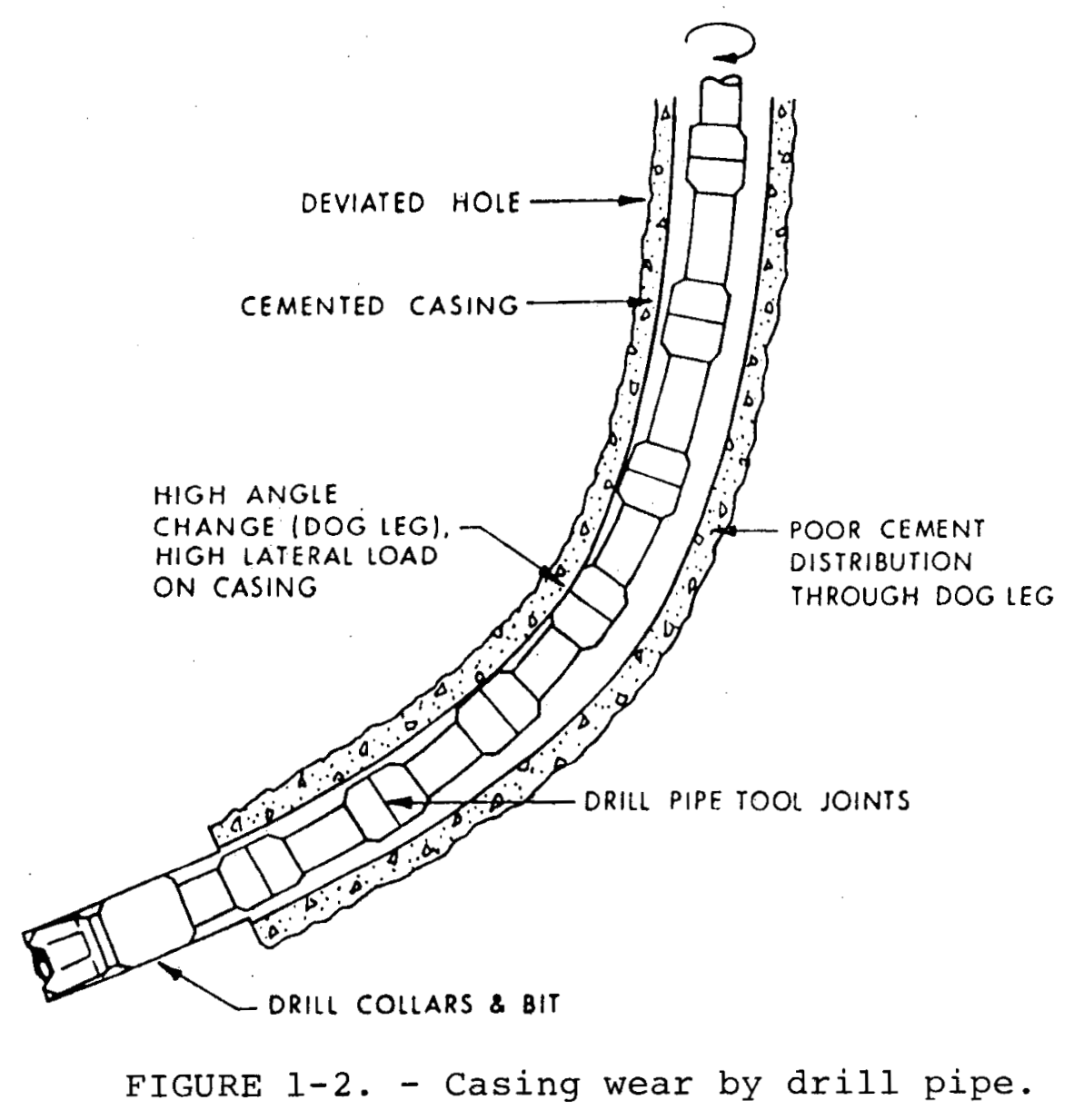

Operators consider surface pipe to be practically sacrificial due to wear, and highly subject to collapse from subsequent theremal stresses or formation movement, so they routinely install tieback strings as the final string, after the liner has been installed (see Figure 1-3).

However, the liner is also subject to drill pipe wear and can be severely weakened. Bit sizes are limited below surface pipe so that the clearance between the new hole and liner is necessarily restricted. Before the cement slurries are circulated, the mud which was used to drill the well is thickened by the geothermal heat. Thus cement circulation is made difficult by the heat thickened mud which is reluctantly displaced. Any pressure buildup may fracture weak zones and cause lost circulation.

Note in Figure 1-3 that casings are cemented solidly. This is an attempt to restrain thermal elongation and shortening by temperature cycling. However, the design is only as good as the cement job. Any voids or channeling allow contact with external water and allow elongation which can create columnar 


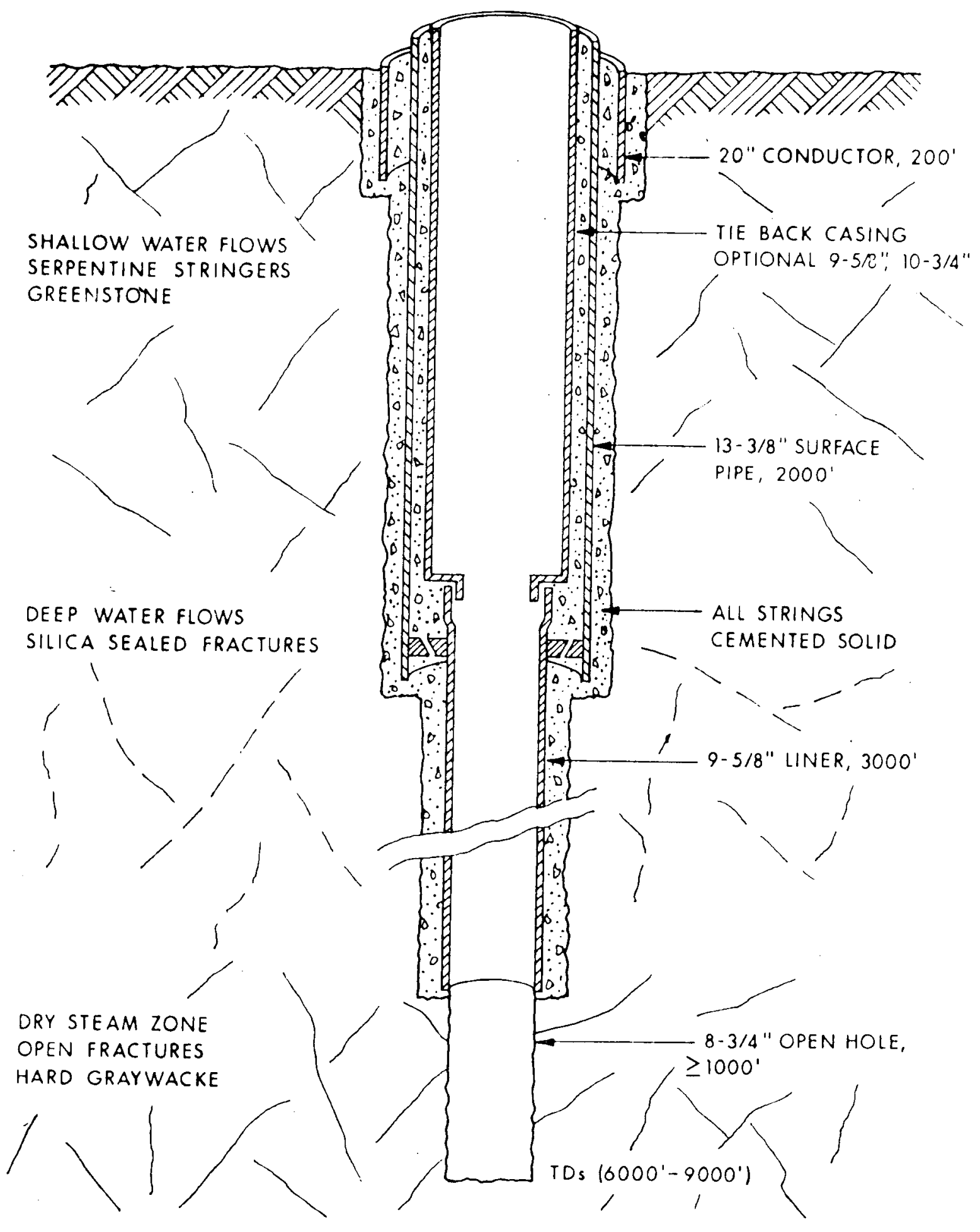

FIGURE 1-3. - Modern Geysers' casing program. Tie-back casing is not used in all producing wells. 
buckling if the temperature rise exceeds a critical amount. ing costs are very high in The Geysers due to a variety of reasons including the requirement to prevent or repair all lost circulation problems before attempting to cement.

Any water entrapped within cement in overlapping sections of casing can cause serious problems as it heats due to subsequent hot fluid flow. The concept is illustrated in Figure 1-4, which also shows other casing wear and failure modes.

After the open hole is air drilled, steam wells are difficult to control. Note in Figure 1-3 that the casing is wide open directly to the formation prior to capping the well. If the well were to flash, the casing would be exposed directly to a rapid transient temperature rise which could cause thermal buckling if there are any unanticipated cementing faults. A typical wellhead used to control such a well is shown in Figure 1-5. Here the well has been "killed" with clean, cold water, the surface casing has been cut off, and the wellhead is welded directly onto the tieback string. The bottom "master valve" is the key control or shut-off device on the well. It is rarely operated, and never against open flow, to prevent seat cutting.

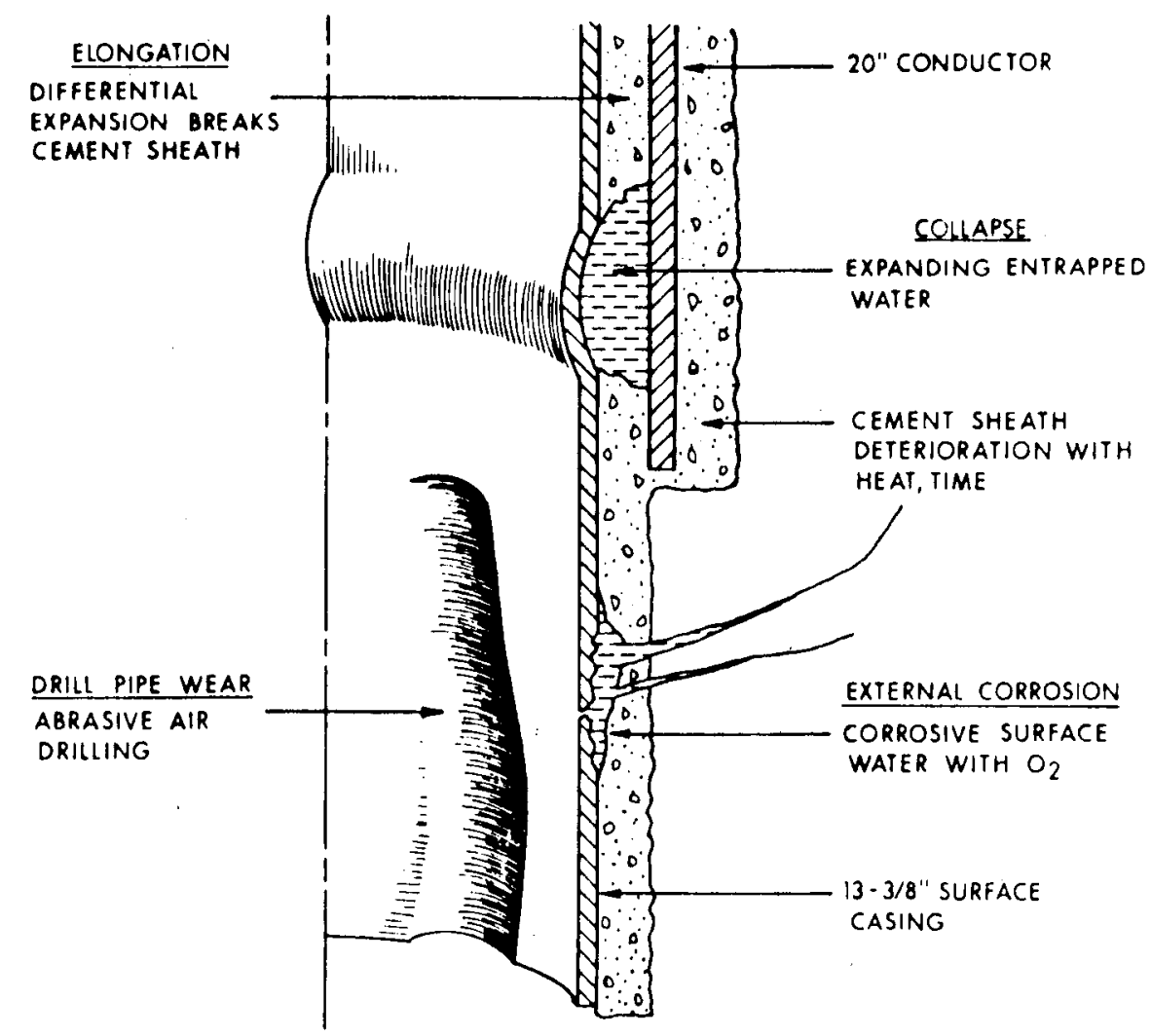

FIGURE 1-4. - Casing problems that can be more severe in geothermal wells due to high temperatures and large diameter strings commonly used. 


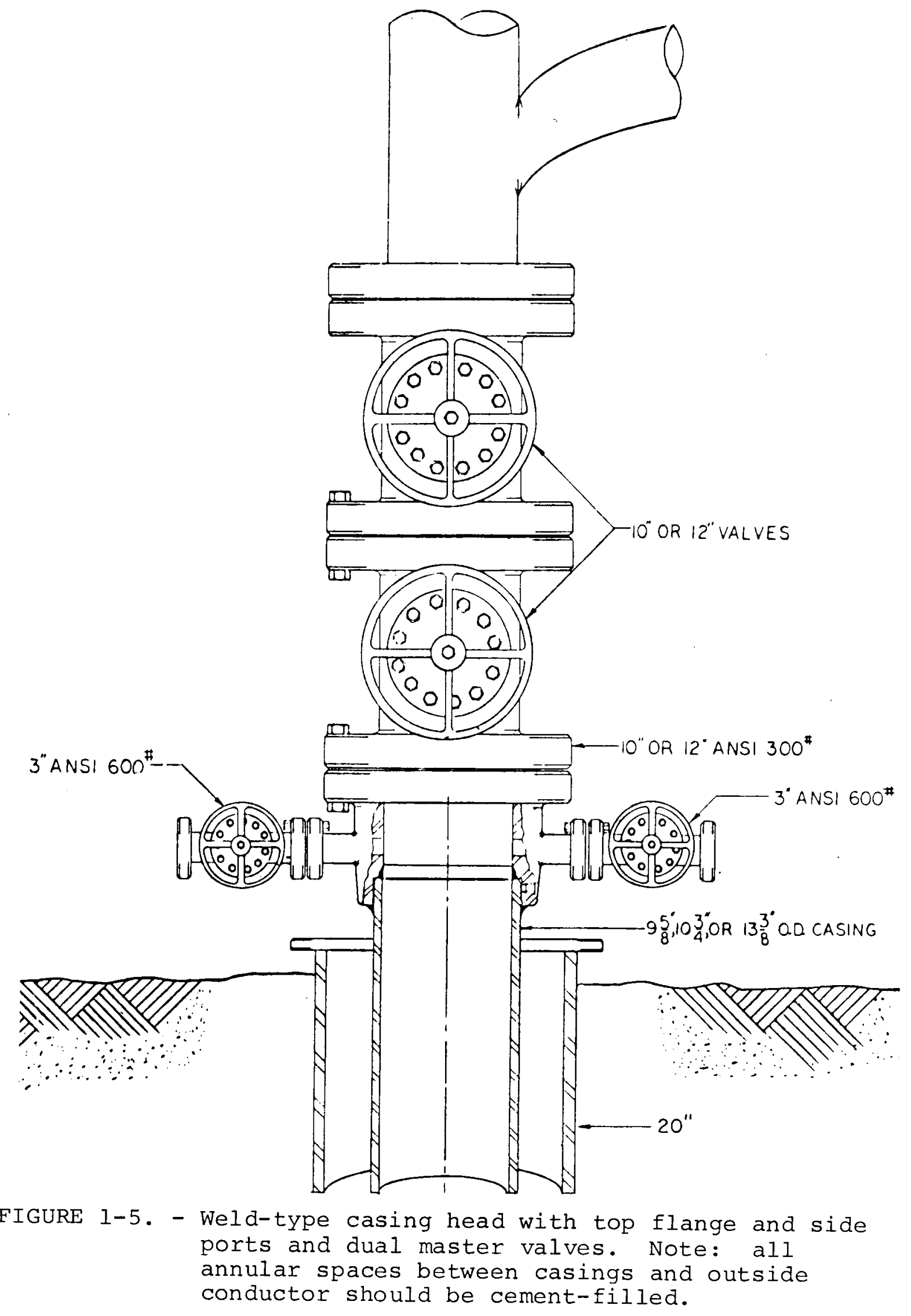


Open hole completions require no perforating. Hot, dry holes are difficult to log for temperature or fracture evaluation. Packers cannot be set to isolate hole sections for cleanout. Wells are kept hot by bleeding small volumes of steam so cyclic thermal stressing is minimized. Also, constant temperatures minimize differential movement of casing strings and practically eliminate need for expansion-type wellheads, which will be discussed further.

\subsubsection{Hot Water Wells}

Wells in which the reservoir fluid is hot or superheated water represent a great variety of downhole conditions and resource applications. For example, individual wells with reservoir fluids that are not commercially attractive for power generation are being completed by various organizations for use as supplemental building heat or for drying fish, vegetables, etc. Temperatures in such wells would range from $150^{\circ}$ to less than $300^{\circ} \mathrm{F}$.

Because $250^{\circ}$ to $300^{\circ} \mathrm{F}$ is the upper range of reliable operation of routinely available oilfield products and services, few problems are expected in these wells due to equipment or material limitations. Product applications would include logging and perforating; setting and releasing packers, bridge plugs and treating tools; valve packing and other elastomer seals. However, due to trace amounts of corrosive gases, high mineral content and elevated temperatures, corrosion and scaling will be continual operating problems.

Typical problem wells are those drilled into superheated brines ( $300^{\circ}$ to $\left.6500 \mathrm{~F}\right)$ which geologically are located in moderately deep ( 4000 to $8000 \mathrm{ft}$ ) reservoirs created by entrapment of aquifers below a "caprock" and above a deeply buried magma body. The caprock, actually a thickness of overlying formation in which fractures or natural permeability have been filled by eons of mineral precipitation, seals the target aquifer from hydrostatic pressure (see Figure 1-6). Thus, typical target reservoirs are "underpressured" and will not flow or support a full column of cold water.

Ramifications of this phenomenon are that wells must be drilled carefully to avoid overbalancing with mud and losing fluids. Also, since water wells are completely mud-drilled, productivity can be severely impaired by mud invasion. Also, the formation lacks "pressure" energy to clean itself, as would a high-pressure oil or gas well.

To make wells produce, the densjty of the wellbore column is first reduced by injection of air or nitrogen. Then hot water inflow warms the column and a definite density difference is generated. The hotter the column becomes, the more it flows 

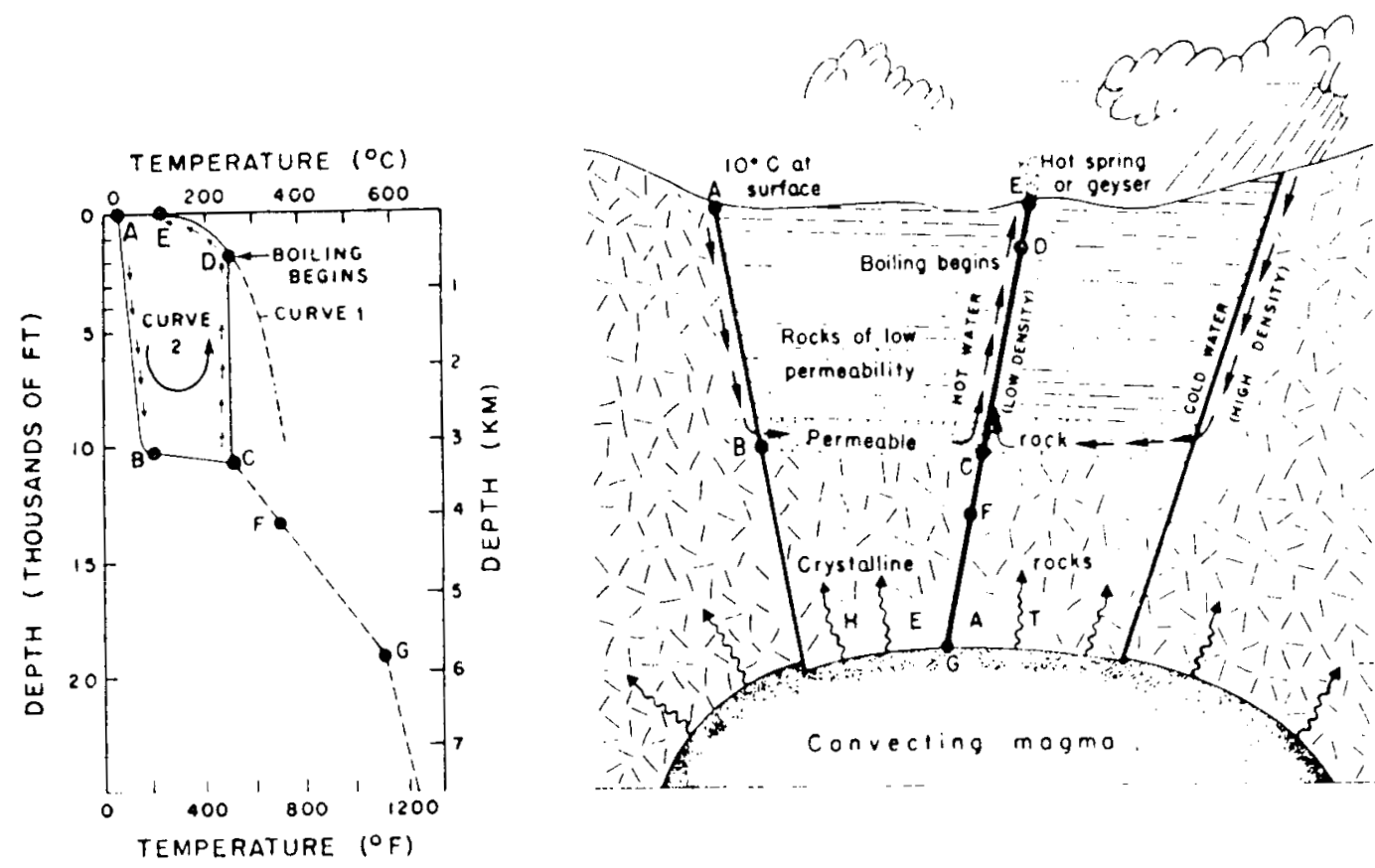

FIGURE 1-6. - Model of a high-temperature hot-water geothermal system. Curve 1 is the reference curve for the boiling point of pure water. Curve 2 shows the temperature profile along a typical circulation route from recharge at point $A$ to discharge at point $\mathrm{E}$.

until pressure somewhere near the surface reaches the saturation point and flashing occurs as shown in Figure 1-7. If not controlled, the entire column may eventually flash.

An important concept is that the high mineral content, superheated brines are highly. susceptible to mineral precipitation under changing pressure conditions. Supersaturated solutions may actually precipitate hard calcium carbonate scales within the formation, several inches away from the wellbore if pressure drop occurs over an internal "skin effect" caused by mud, clay or particle plugging. Scale may also form on the slots of slotted liners or, in cemented pipe, within the very critical "perforation tunnel" through the casing wall and cement sheath (see Figure 1-8).

In conventional wells, acids may be used to remove such plugging materials. However, geothermal conditions make acidizing expensive and potentially damaging. Acid inhibitors for protecting tubulars are temperature-limited and boreholes may have to be cooled by circulation to $250^{\circ} \mathrm{F}$. Further, the acids can remove natural grain bonding minerals from directly around the borehole and contribute to sand influx or wall sloughing. 


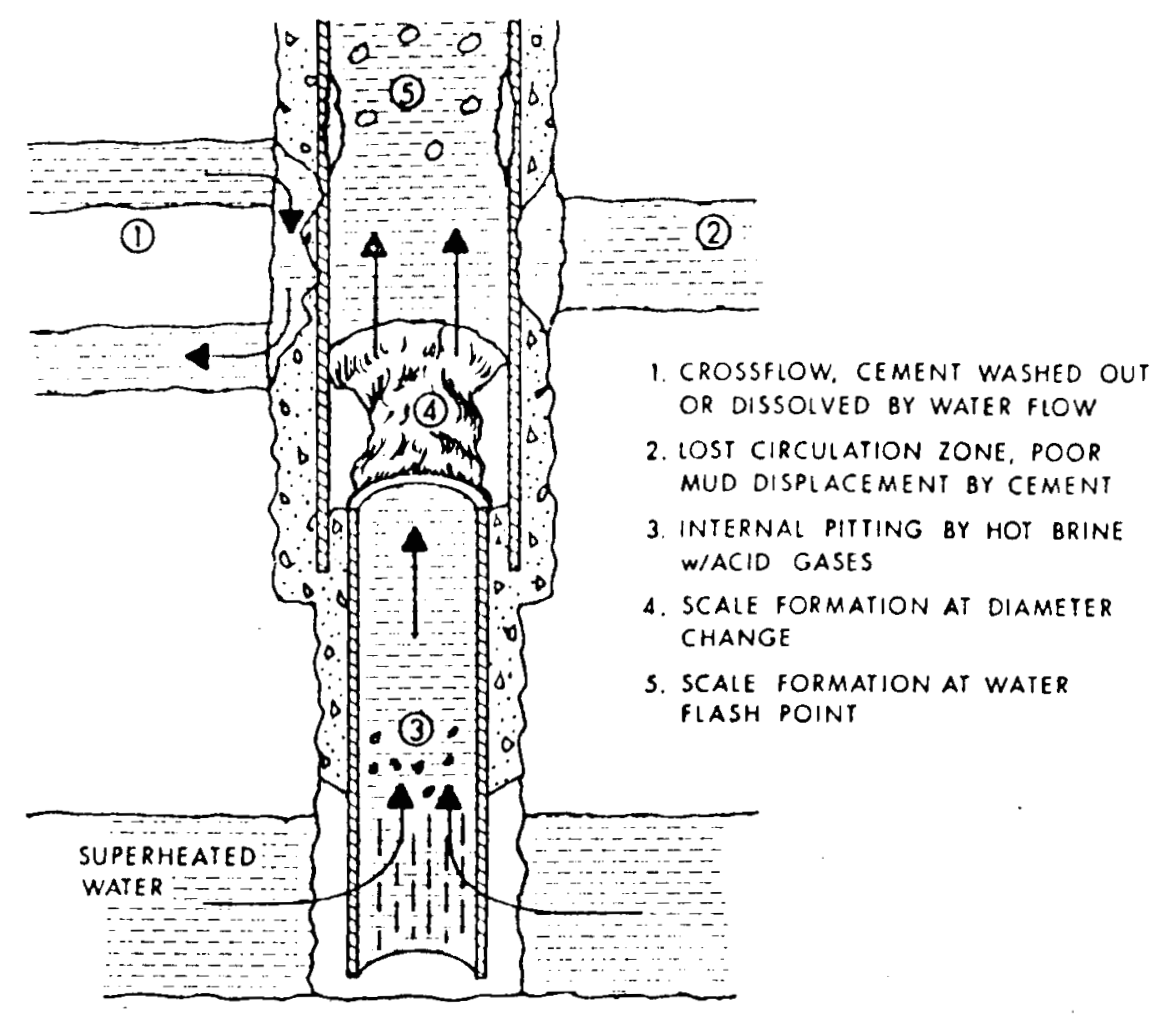

FIGURE 1-7. - Typical downhole problems in hot water wells.

Hot water aquifers are characterized by very thick sand bodies and interspersed shales. Up to 2,000 ft of producing interval may be opened to flow to allow rates of 20,000 to 70,000 $\mathrm{bbl} / \mathrm{day}$.

In thinner, cooler zones, operators may choose to cement casing or liners across the entire productive zone, then selectively perforate desired sands. Such methods are possible if open hole evaluation logs are run in holes that are cooled by mud circulation. Widely applicable tools can operate in such environments to perhaps 350OF. Chevron USA, for example, will attempt to complete the Heber (Imperial Valley) area in this manner. Benefits of cementing and perforating are that the zones can be selectively tested, treated, or stimulated by setting packers between perforated intervals (see Figure 1-9).

For hotter reservoirs, that is, $350^{\circ}$ to $650^{\circ} \mathrm{F}$, and for long intervals, the cost of jet-type perforations at four holes per foot becomes excessive. Perforating alone may cost $\$ 250,000$. 


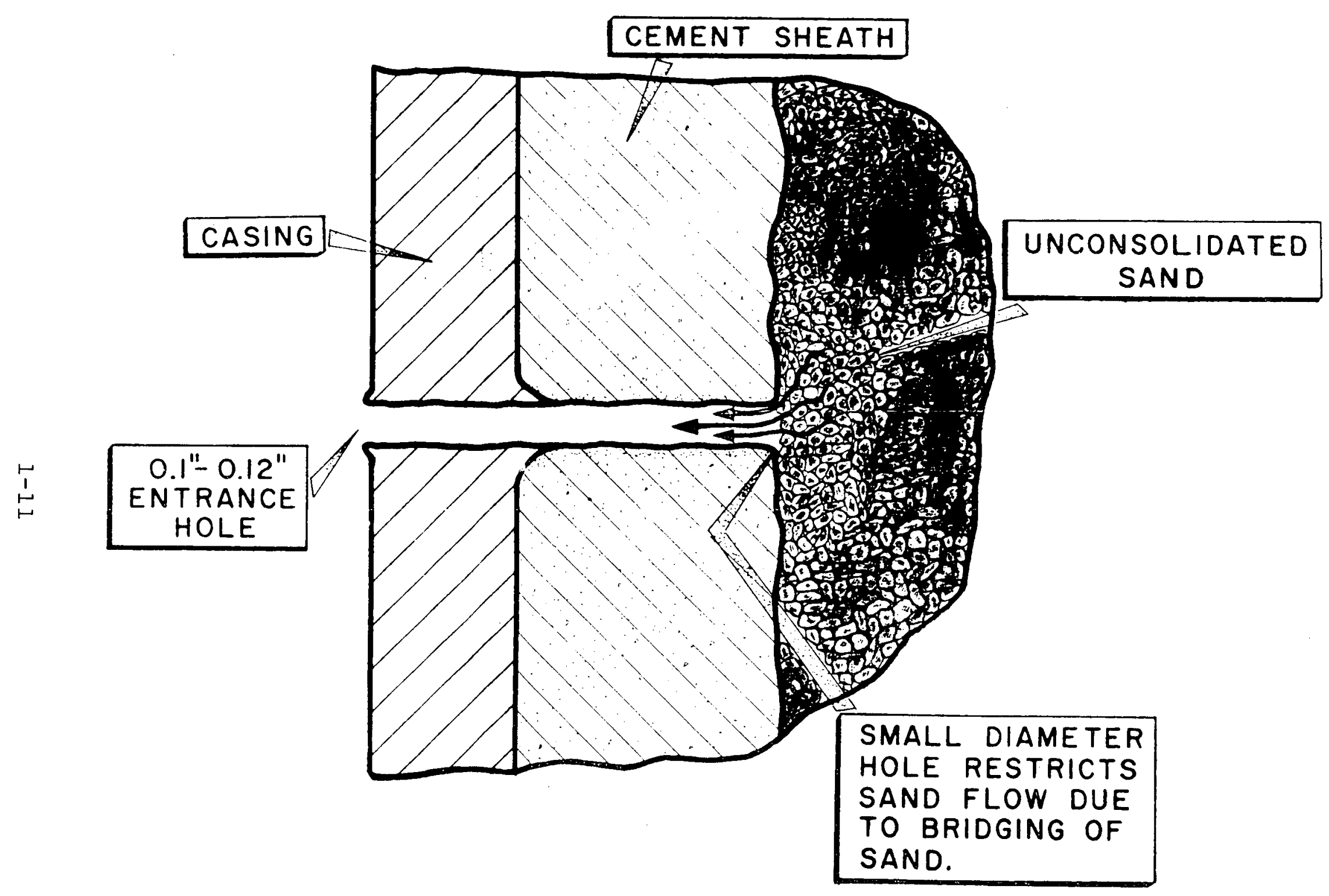

FIGURE 1-8. - Perforation tunnel through pipe and cement. 


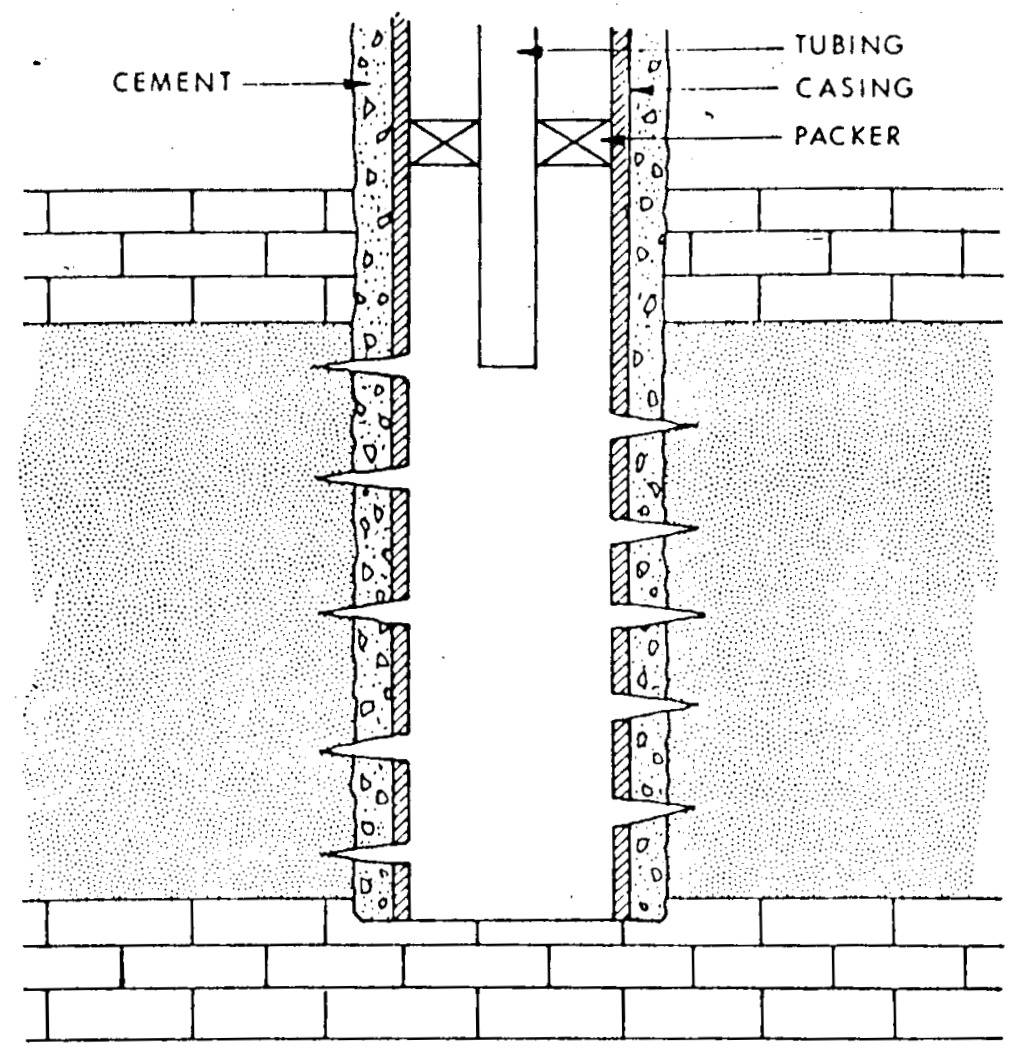

FIGURE 1-9. - Schematic of cemented and perforated casing.

Also, due to heat, jet charges lose efficiency, and reducedperformance, higher-cost explosives are required. Example tolerances for available explosives are:
a. $R D X$
- $340^{\circ} \mathrm{F}$ for $1 \mathrm{hr}$
b. HMX
- $400^{\circ} \mathrm{F}$ for $1 \mathrm{hr}$
c. Picryl sulfone $-420^{\circ} \mathrm{F}$ for $1 \mathrm{hr}$
d. HNS
$-500^{\circ} \mathrm{F}$ for $1 \mathrm{hr}$.

Operators commonly choose to run long liners that are hung in the casing string on slip-type hangers, with the lower end above the hole bottom. The liners may be completely uncemented and slotted over the entire length, as are most california-type sand control liners, or they may have the upper part 
cemented with such cement extending into the overlap area, as shown in Figure 1-10. A great variety of casing and liner configurations is possible.

Because pressures are low and wells are relatively shallow, tubulars can be made of lower-strength carbon steels. Due to increased corrosion potential in quenched and tempered metal, particularly $\mathrm{H}_{2} \mathrm{~S}$ stress corrosion cracking with ever-present sulfur, N-80 grade steel is the hardest material in use, but one operator (Comision Federal de Electricidad, Cerro Prieto, Mexico) would prefer not to have to use even that grade. Stainless steels, supposedly resistant to electrochemical corrosion, are highly susceptible to chloride stress cracking. An example water analysis of a salton sea well which was $6080 \mathrm{~F}$ at only $4200 \mathrm{ft}$ is shown in Table $1-1$.

Because hot water wells in the United States cannot be flowed until, and unless, provisions are made to dispose of or continuously reinject cooled brine (for reservoir performance), these wells are subject to cyclic temperature changes, for example, between initial testing and power plant installation.

Due to temperature changes and differential elongation and contraction of concentric strings of tubulars, such as a tieback within surface casing, two strings may not be rigidly connected within the wellhead, as they would be in an oil or gas well. Special. geothermal wellheads with expansion spools and heatresistant packing are used as in Figure l-11.

It is erroneously believed by some that cementing casing constrains thermal movement and thus prevents stress failures. This is not the case, as constraining pipe actually creates stress as pipe attempts to elongate (or contract). The basic formula for such stresses is:

$$
S=\alpha \Delta T E
$$

where

$$
\begin{aligned}
S= & \text { stress, lb/in. } \\
\alpha= & \begin{array}{l}
\text { coefficient of linear expansion for steel, } 6.9 \times 10^{-6} \\
\text { in./in. } \mathrm{F}
\end{array} \\
\Delta \mathrm{T}= & \text { temperature change, }{ }^{\circ} \mathrm{F} \\
\mathrm{E}= & \text { steel modulus, } 29 \times 10^{6} \mathrm{lb} / \mathrm{in.}{ }^{2}
\end{aligned}
$$




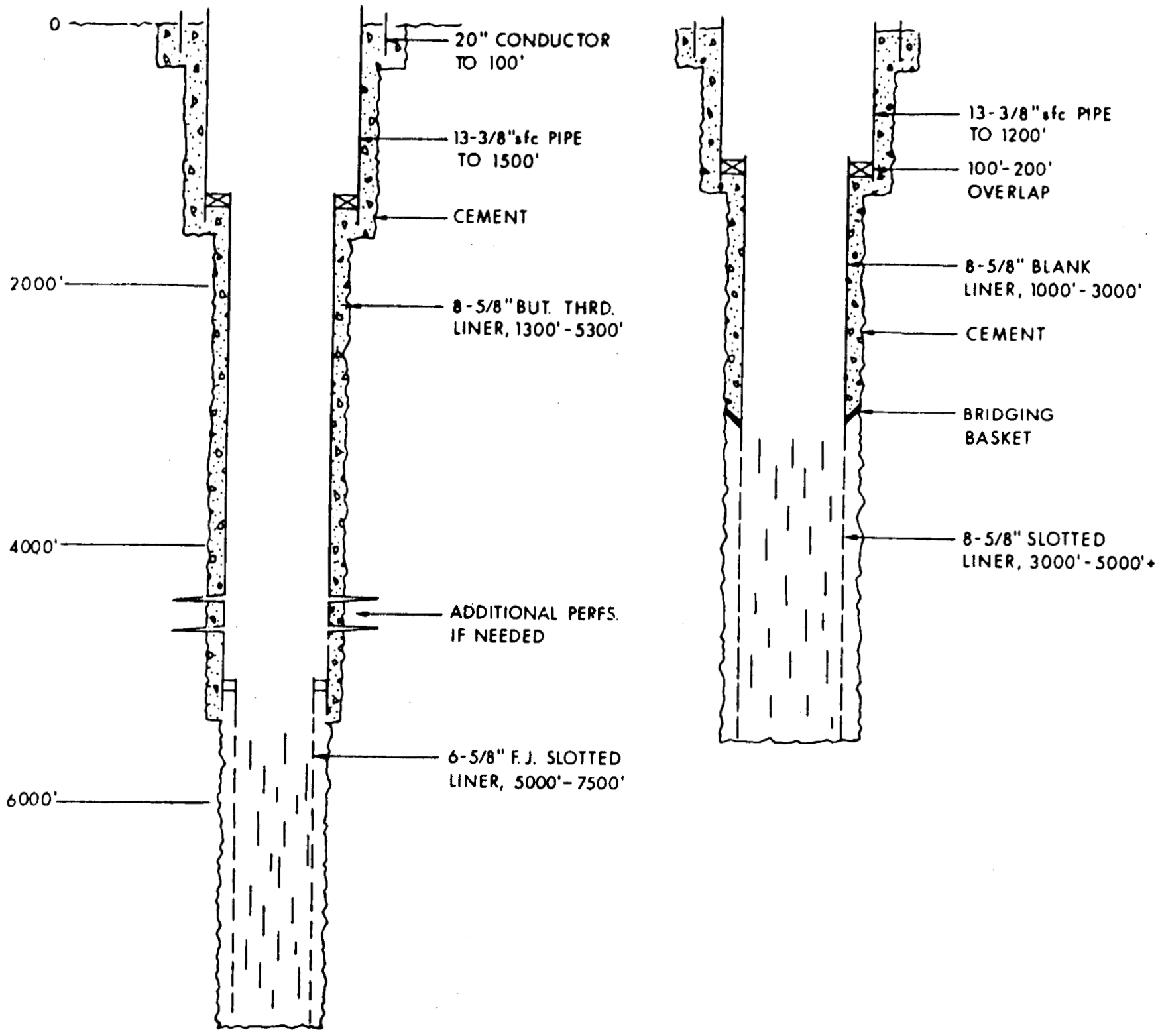

$8000^{\prime}$

FIGURE 1-10. - Typical Imperial Valley completions for two well depths. 


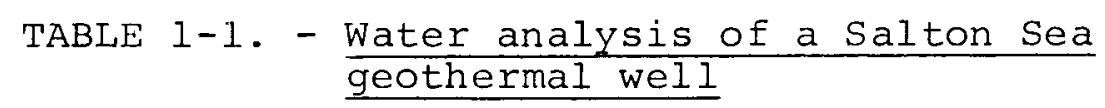

\begin{tabular}{|l|c|l|r|}
\hline Constituent & $\begin{array}{c}\text { Parts per } \\
\text { million }\end{array}$ & Constituent & $\begin{array}{c}\text { Parts per } \\
\text { milion }\end{array}$ \\
\hline Sodium & 53,000 & Manganese & 1,370 \\
Potassium & 16,500 & Lead & 80 \\
Lithium & 210 & Zinc & 500 \\
Barium & 250 & Copper & 3 \\
Calcium & 28,800 & Silver & $<1$ \\
Strontium & 440 & Rubidium & 70 \\
Magnesium & 10 & Cesium & 20 \\
Boron & 390 & Chloride & 115,000 \\
Silica & 400 & $\sum$ CO 2 & 500 \\
Iron & 2,000 & $\sum$ S & 30 \\
\hline Total dissolved & solids & & 259,000 \\
\hline
\end{tabular}

Note that with temperature changes of $350^{\circ} \mathrm{F}$, which are possible, stress will approach $70,000 \mathrm{lb} / \mathrm{in.}{ }^{2}$. This exceeds the yield strength of commonly used J-55 (55,000 lb/in. $\left.{ }^{2}\right)$ casing. The behavior of such stresses is shown schematically in Figure 1-12. Field examples of failures are parted couplings where API round tapered threads have been compressed and yielded, then pulled apart in tension when the distorted coupling was stressed by severe quenching. 


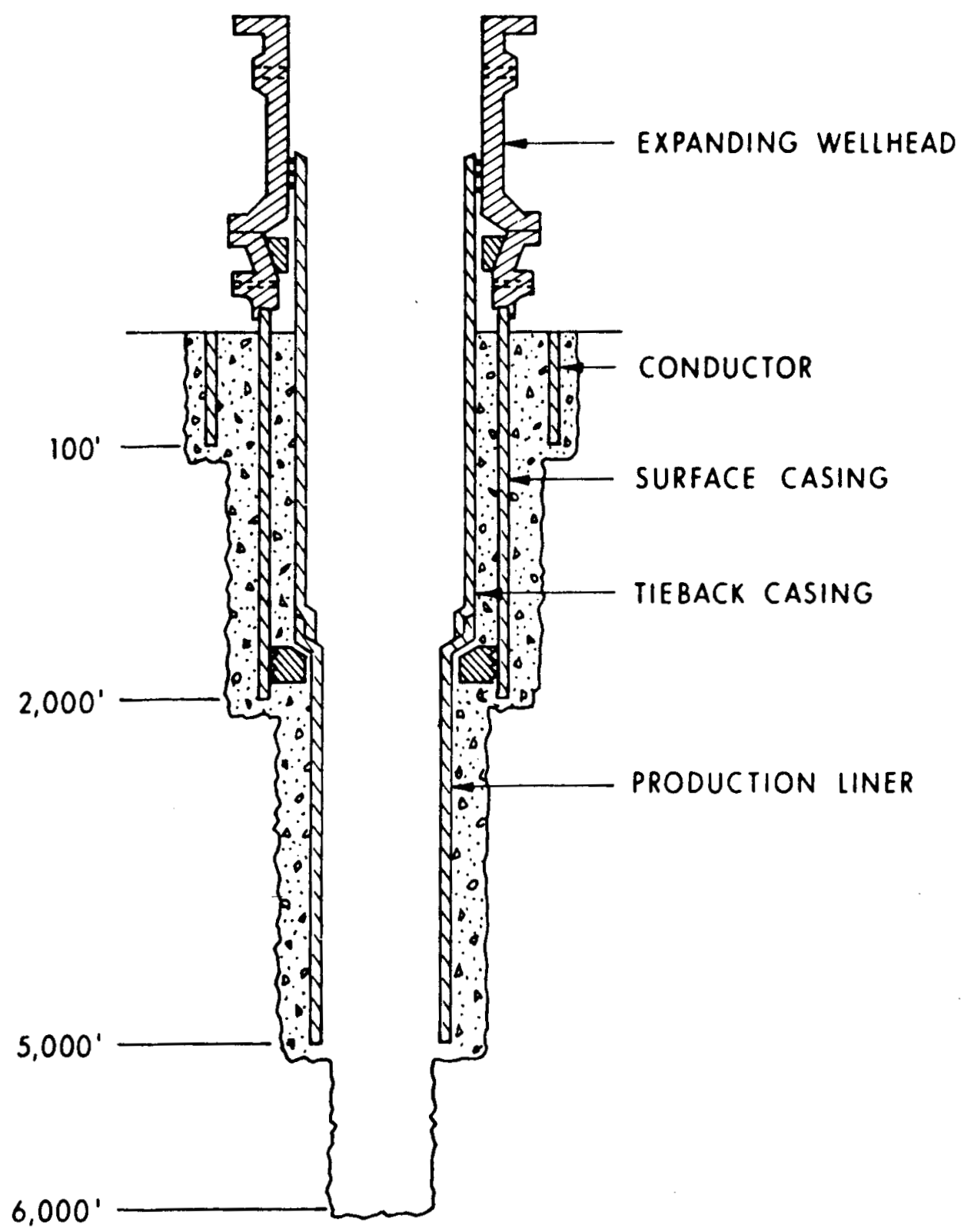

FIGURE 1-II. - Hot water producing well with expansion spool on wellhead. 


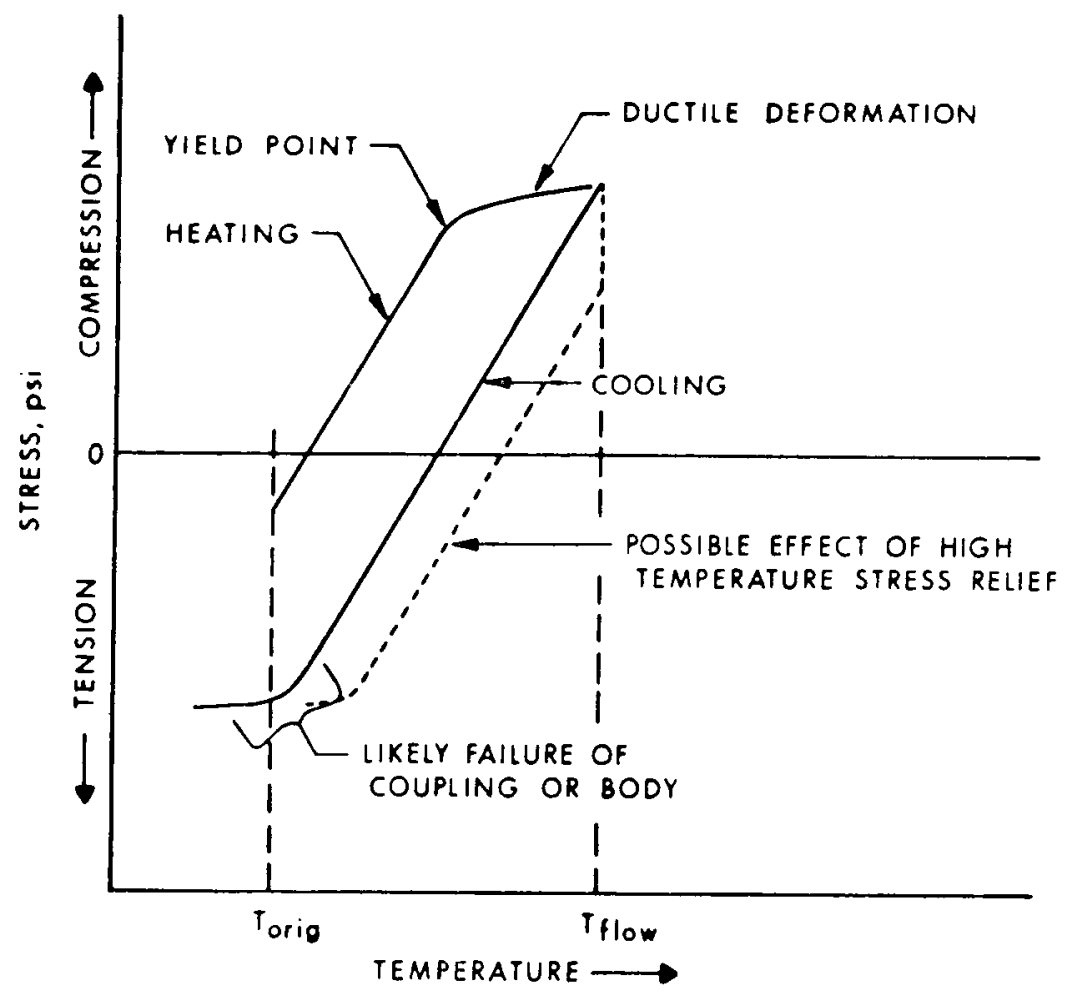

FIGURE 1-12. - Stress behavior in solidly cemented casings. 



\section{SURVEY OF EXISTING EQUIPMENT AND NEEDS}

\section{Summary}

The purpose of this section is to survey existing equipment and needs and identify major areas where there is potential for equipment and methodology improvement.

The survey identified five major areas to investigate:

a. Cementing equipment limitations

b. Sand control

c. Corrosion prevention

d. Slotted Ziner limitations

e. Well perforator limitations.

This investigation identified a variety of problems which could be solved with technology.

Three problems were identified and analyzed in detail in section 3 to indicate the type of technical approach which couzd be used to address these eeemingly unmanageable technical problems.

\subsection{Cementing Equipment Limitations}

\subsubsection{Introduction}

In geothermal wells, optimum primary cementing is critical to well performance for two basic reasons. First, cement is a basic component of the downhole equipment installation in all parts of the hole, literally from top to bottom (except for those wells completed in open hole). Unlike oil and gas well completions, thermal stresses and unique problems with hot aqueous fluids can cause well failure at any depth and at any future time, if initial cementing of any tubular string was improperly done (l)*.

Secondly, productivity or injectivity may be reduced if zonal isolation is not achieved through proper displacement of the drilling mud and installation of best available cement compositions.

Any cementing operation consists of two basic functions:
a. Slurry composition preparation
b. Slurry placement.

\footnotetext{
*Underscored numbers in parentheses include references to sources of material. These references are included as section 4 of this report.
} 
Slurry compositions have been studied extensively and new developments may or may not be forthcoming. However, operators accept present formulations of high-temperature cements as the best available and concern themselves primarily with ensuring quality control and proper field mixing procedures.

Conversely, the slurry displacement function requires cooperation of both operator and service company to be successful. The operator must attempt to provide a properly drilled borehole with adequate annular clearance, properly conditioned mud, and well-designed tubulars containing recommended equipment (such as centralizers and wall cleaning devices). The service company supplies downhole and surface cementing equipment, and expertise to pump and control the cement and any preflushes and displacement fluids.

Failure of downhole cementing equipment may or may not adversely affect job results in an immediately identifiable manner because not all downhole equipment serves a "critical" purpose. Basically, primary cementing involves pumping fluids around a "U-Tube." The job could be accomplished in simple casing strings with no downhole cementing equipment.

Examples of "noncritical" cementing tool functions are the ability of a bottom or top wiper plug to scour efficiently drilling mud or cement from the casing wall, and the ability of a centralizer bow to achieve 100 percent centralization of the pipe. Also, it is common for a float collar valve to stick open and require that pressure be held to prevent slurry backflow after displacement. Such failures may lead to poor future well performance, yet never be identified as the problem source.

Conversely, certain equipment must function as designed or job failure and a seriously impaired completion will result. Examples include failure of a stage collar to open, plugging of a bottom wiper plug, failure of a liner wiper plug, and failure of an inner string seal on the bottom of the drill pipe.

Because downhole cementing equipment is rarely recovered intact, failure mode analysis is seldom possible. Thus, equipment reliability can only be assured through proper engineering design, surface testing, and evolutionary changes prompted by obvious failures in wells that extend previously proved ratings.

At the present state of geothermal development, cementing equipment is being subjected to downhole environments more severe than typical oil and gas well conditions. Equipment furnished for the fledgling geothermal industry has not been rigorously engineered for the special requirements. Typically, "geothermal" trim is substituted for conventional components, instead of basic redesign. In most cases, this has been satisfactory or, at least, few complaints have been registered because: 

a. There are relatively few geothermal wells.
b. Most cementing applications occur in cooler (shallower) hole portions or in circulated, mud-cooled deeper intervals.

However, persistent reports of equipment limitations come from Cerro Prieto field where the operator is drilling and cementing in deeper, hotter horizons than those yet encountered in field development phases in the United States (2). This is taken as proof that existing equipment is very close to limits of reliability in United States fields and may not be acceptably reliable for near-future development of deeper, hotter areas such as the Salton sea and deeper Heber field zones. Also, greater emphasis is being placed on cementing and perforating in fields such as East Mesa as a method which allows more efficient selective testing and treating. Greater need for efficient, channel-free placement will require optimum performance from equipment -- not merely the ability to complete the job without mishap.

The conclusions presented in the above introductory discussion and a useful description of basic cementing equipment appear in a recent report, "Primary Cementing Equipment and Critical Development Needs as Applied to Geothermal Wells," prepared for Sandia Laboratories by Completion Technology Company (CTC) under Contract No. 46-0173. The specific component tests conducted by Foster-Miller Associates, Inc. (FMA), as described later in this report, fulfill a portion of one recommendation made in the above-noted document.

In the following subsection, typical cementing tool applications are illustrated schematically, without regard for similarity to any particular commercially offered item. Potential limitations are indicated, as may occur in severe geothermal well environments. The primary objective of these illustrations is to explain and justify concurrent contract efforts to examine performance behavior of certain components and materials. Also, the discussion may stimulate user or designer interest such that suppliers may recognize limitations in their own designs and recommend modifications. It is fully recognized that equipment may be available that does not have limitations as suggested.

\subsubsection{Potential Failure Modes}

Since downhole cementing equipment is simplistic and involves few "moving parts," it is assumed that failures will be the result of material composition limitations under unique stresses of the geothermal environment. 
It is commonly known that elastomers have definite temperature limitations. It is also known that steel loses strength rapidly above $700^{\circ} \mathrm{F}$. What is not known, and what has not been tested by industry suppliers (simply because a need has not been defined), is what happens to metallic and nonmetallic components at elevaied temperatures, in the presence of aqueous fluids with strong chemical properties.

The following discussions illustrate some component or material "potential" failure modes. In other contract efforts described later, some component materials were briefly analyzed by engineering methods and simple physical tests were conducted to attempt to verify the mathematical models. The objective is to prove a need to pursue much more detailed study or to stimulate actual development of a new material. In many cases, the preliminary studies may convince an operator or a supplier to recommend better materials or components that are currently higher-priced alternatives.

Two primary studies were attempted by FMA on representative cementing equipment. Downhole effects on spring steels were analyzed in a simulated well casing to assess deterioration due to frictional heating and wear. In addition, simple wear analysis was made of a sample cementing wiper plug to attempt extrapolation to field conditions. The following failure mode discussions indicate how data from these tests are relevant to existing field operations.

\subsubsection{Wiper Plugs}

It is convenient to think of a rubber plug as a piston that is driven by one fluid to move another fluid. That concept would lead the casual investigator to assume that differential pressures force the flexible fins and/or cup edges against the casing wall to create a large loading force. Under that assumption, it would seem unlikely that the elastomer surface could survive even a short distance without severe wear and deterioration.

It is probably more correct to visualize the plug as floating in a pressurized fluid. One fluid actually moves the next as it would if the plug were missing. Being completely surrounded by equal pressure, the fins may actually engage the wall with only compressive forces caused by the interference fit between the cementing plug and the casing inner diameter. Wear of the plug fins will be a function of the elastomeric properties of the fins and the environment to which the fins are exposed (temperature, abrasive content of the mud, etc.) as the plug is pushed downhole ahead of or behind the cement. Because it is unlikely that the cement plug supports any pressure differential between the cement and mud, wear of the fins is strictly due to frictional wear as the plug is shoved downhole. 
Plug functions, as illustrated in Figure 2-1, are interesting to speculate upon. Basically, the plug separates two different fluids to minimize mixing. To do this, the fins are assumed to wipe the inner wall cleanly. Note in Figure 2-la that such a function would disrupt the normal laminar flow regime in which outer layers move more slowly. Immediately below the plug, all fluid layers have equal velocity. Thus, to compensate, an increased velocity is induced midstream at a lower point. The effect then is to create a type of "turning-over" cell below the plug.

In Figure 2-1b, this modified flow profile is shown to include a film of more concentrated particles such as may accumulate in muds contacting the hot casing wall for some time.

The significance of the various wiping and flow profile scenarios is that:

a. If the wiping surface remains efficient, the lower plug will accumulate a significant volume of mud ahead of it which will be harmlessly displaced ahead of the cement column.

b. If the lower plug is left out (not uncommon), or does not remove mud film due to heat and wear, the top plug may collect that mud volume and cause it to be deposited around the critical shoe joint area in the annulus.

c. If either plug wears and bypasses a significant portion of the wall film, fluids may mix, or displacement calculations may be incorrect, and much contamination will be left in the casing.

In Figure 2-1c, the sealing function is illustrated. The bottom plug, in an extreme case, may deteriorate (soften) with heat, or, the metal insert may fail such that it plugs the baffle or float collar. A failed top plug would not effect a pressure seal when it landed on the bottom plug. Junk or debris on the lower plug could prevent seal formation. Such rare but possible failure modes are not limited to geothermal wells, and they would be more attributable to carelessness than tool limitations.

Elastomer seals are also used on other types of cementing plugs including those on staging tool opening and closing plugs and on flapper-type float valves. However, geothermal trim usually minimizes risk of seal failure by "backing up" any elastomer seal with metal-metal "no-go" design. Also, many sealing faces are aluminum, which gives a more durable primary sealing surface. 


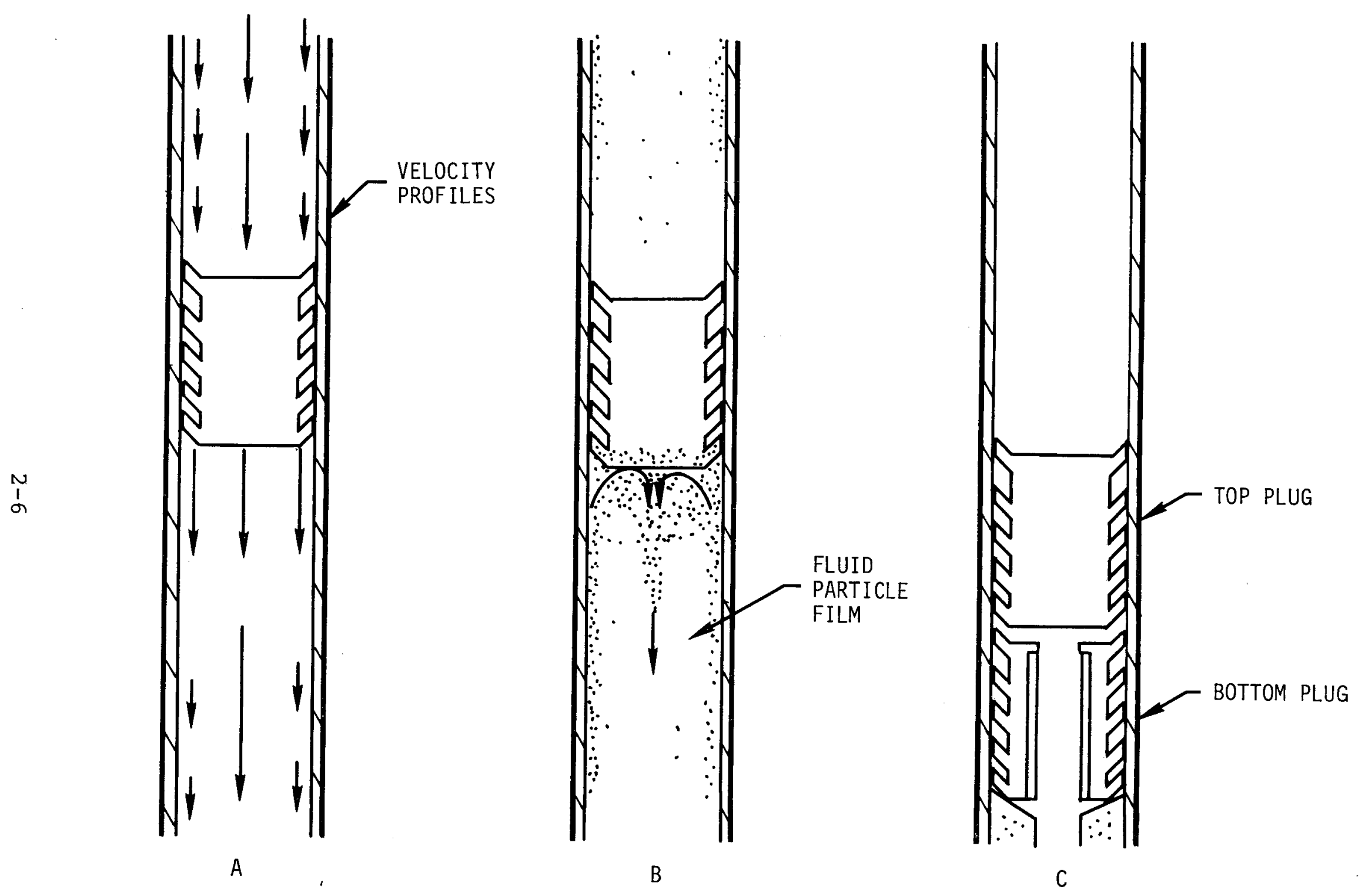

FIGURE 2-1. - Considerations for cementing wiper plug functional analysis. 


\subsubsection{Spring Steel (Centralizer) Performance}

Failure of a centralizer bow has never been noted as a problem in geothermal well cementing in any investigations conducted by CTC. CTC did, however, experience and document breakage of centralizer bows during an attempted installation of inflatable packers in a Hot Dry Rocks well under sponsorship of Los Alamos Scientific Laboratory. In that well, two bows on separate centralizer units broke cleanly near the center of the bow while lowering a 5-1/2-in. liner into a 9-5/8-in. casing. The broken spring prevented entry of the liner assembly into a swage which reduced casing size to 7-5/8 in. The static well was filled with water and the well system was warmer than a normal well, but not up to geothermal temperature at shallow depth.

Because of this experience and speculation that certain unique stress effects may be induced into spring steels under geothermal conditions, FMA conducted simplified but detailed investigations into potential failure modes. These studies concentrated on the contact point of the bow spring with the outer casing wall to evaluate the possible weakening effects of point loading and localized heating and friction effects. The following discussions present an overview of the potential problem using illustrations of components applications downhole.

Figure 2-2 schematically represents a centralizer bow moving through an existing casing and into freshly drilled open hole. Example situations would be 9-5/8-in. drilling liner being lowered through $2,000 \mathrm{ft}$ of $13-3 / 8-i n$. surface casing, or 7-in. liner traversing 4,000 ft or more of a 9-5/8-in. driliing liner.

In a reasonably straight hole, centering forces on the inner string will be in the magnitude of 300 to $700 \mathrm{lb}$, depending on clearance between inner and outer casing (or open hole). A high percentage of this total loading will be accepted by individual bows to force them strongly into the inner wall of the casing. Depending on system and bow geometries, the bow-casing contact area may be very small and pounds per square inch loading could be very high. Two factors affect the contact area:

a. The radius of the vertical curve as in Figure 2-2.

b. The relative geoemtries of bow and outer casing crosssections, as in Figure 2-3.

Note in Figure 2-3 that a flat bow would surely give point loading on the edges, inside any casing. Other configurations, such as a curved cross-section offered by at least one supplier, may contact the casing at a central point or in a horizontal line, depending on how closely the two radii match. 


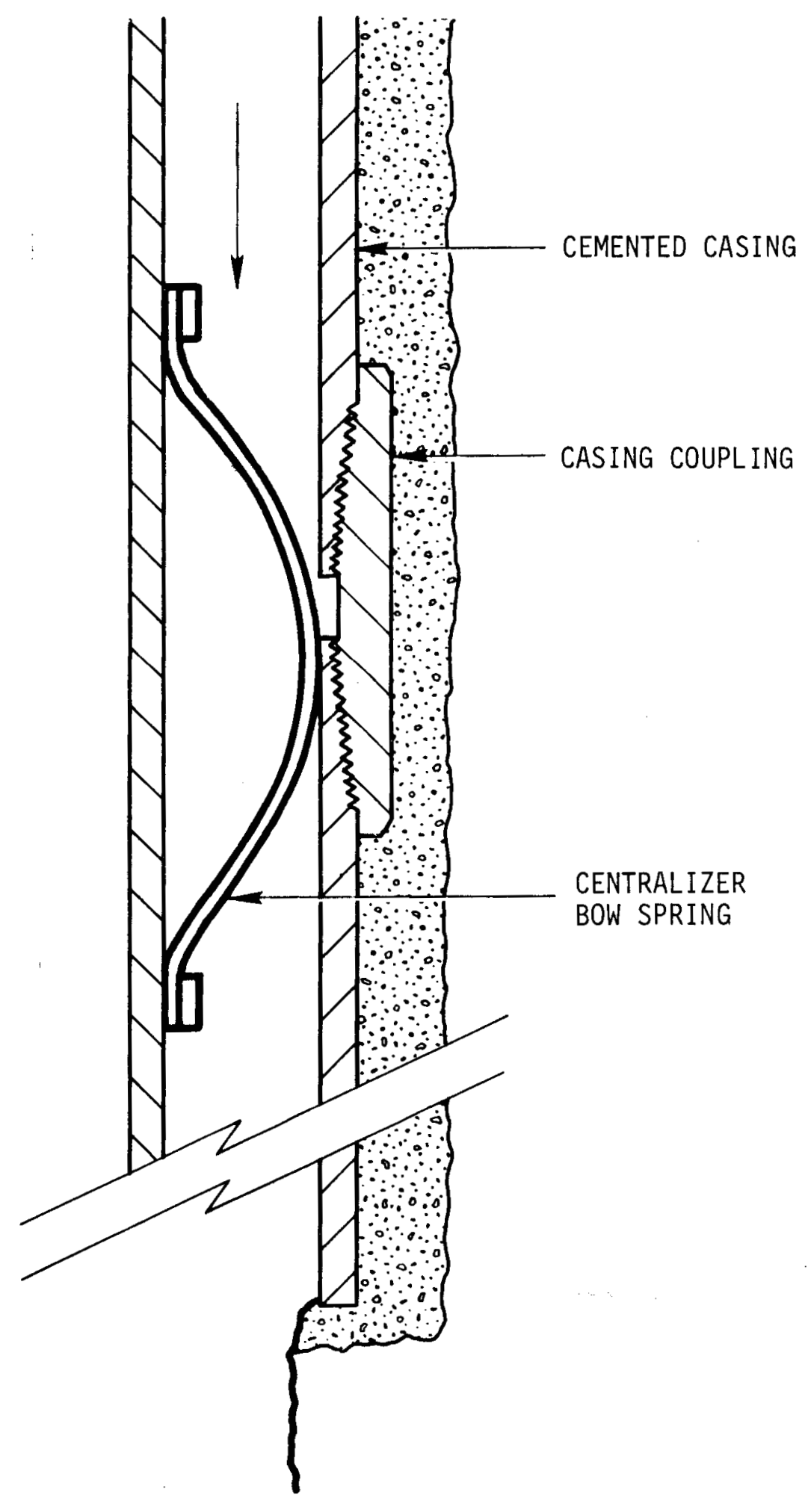

FIGURE 2-2. - Schematic representation of centralizer bow spring moving through casing to open hole. 


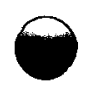

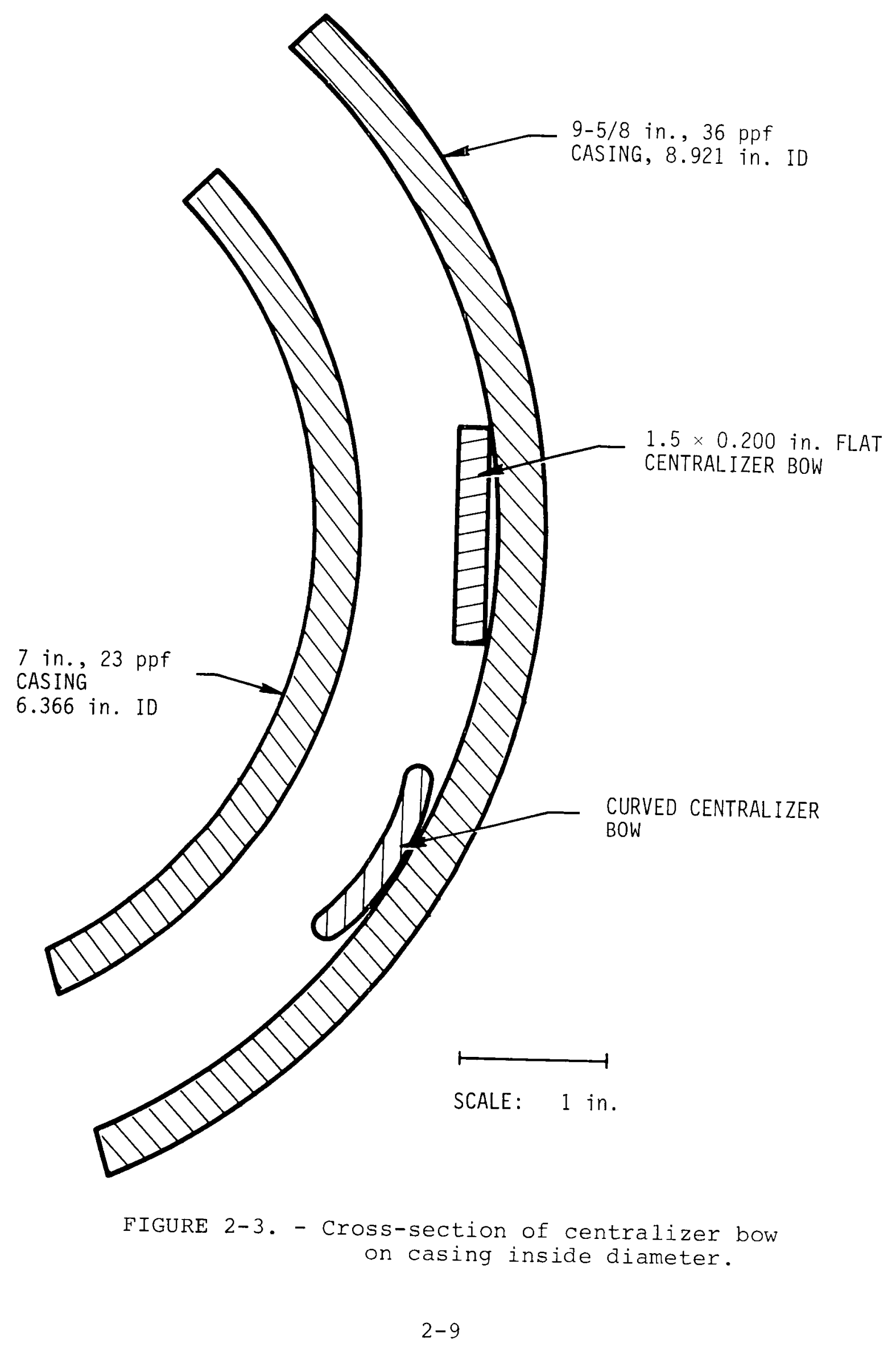


In any case, it is speculated that the strong point loading may generate heat due to friction that could alter the metallurgy of the hard, quenched and tempered spring steel. Internal stresses caused by deformation, plus the added shocks of crossing gaps at couplings or traversing anomalies such as staging collars, then may initiate fracture failure.

Millions of bow springs have been run in oil and gas wells, primarily in drilling mud environments. Chemical efforts combined with downhole heat and more aqueous fluids may be a factor to consider. However, most centralizers utilized in primary cementing would also be in mud-filled and circulated holes. Exceptions may be in Baca Ranch wells, where slotted liners are run into holes drilled with air-assisted water.

If centralizers are adequately spaced, individual units could lose some strength and still provide some standoff, providing they did not break. In a liner overlap area, lack of centralization could result in cement channeling and creation of a mud pocket which could collapse the liner with heating.

It is also noted that drag springs are used as operating mechanisms to set slips on liner hangers and mechanical packers and bridge plugs. Should this be identified as a problem -which it has not, according to CTC contacts -- hard-faced drag blocks may be substituted for bow-type drag springs.

\subsubsection{Stage Collars}

More than one operating company has noted that stage collar opening failure is not uncommon. One such operator is Corporation Federal de Electricidad (CFE) of Cerro Prieto, Mexico. Because wells are deeper in the cerro Prieto field, and long production casings are run there, it is not possible to circulate even lightweight cement slurries very high off bottom without breaking down fractured zones and losing cement.

Also, after running casing, Cerro Prieto wells are circulated for several hours, which would expose the staging tools to prolonged high temperatures which would surely exceed $300{ }^{\circ} \mathrm{F}$ even in the circulating systems. In early jobs, up to three stage collars could be used. It was not uncommon for the first staging tool not to open. Second and third staging tools had an even worse record. These failures have not been documented and no record of failure analysis is available to help analyze the problem.

In a technical paper presented in Albuquerque in January, 1981, specific references to cementing problems in Cerro Prieto were noted (2). Two example quotations are, "Some problems have been solved; others, such as those pertaining to well casing, 
cement and cementing jobs, have persisted," and "Cementing operations have not always been controllable and probably, a solution would be to use light cements, that would tolerate degradation with time, since improvements of cementing collars is apparently more difficult" (ㅁ) .

Cerro Prieto could be considered a "proving ground" for geothermal cementing tools as development operations are deeper and hotter than those in any commercial development in the United states. Thus, any problems occurring in cerro Prieto may face United States companies in future operations, particularly in deeper Imperial Valley wells.

Obviously, a failed stage collar would never be recovered because attempts to operate it would not be made until the casing string containing it is surrounded by cement and thus made irretrievable. Therefore, the following comments on possible failure modes are speculative.

The concept of staging tool operation is illustrated in Figure 2-4. Various styles of tools are available. Several suppliers offer equipment that functions similarly to the schematic shown, with extra features such as additional seals, 1ocking mechanisms and shear pins. One supplier's equipment features a variation of the closing sleeve in which internal casing pressure closes a second internal sleeve, after the first sleeve exposes a pressure access port. Actual drawings of present tools are available in Composite Catalog (Gulf Publishing Company, Houston) or directly from the major service companies.

Most stage tools are operated by two plugs applied in sequence which, when dropped or pumped, effect a pressure-tight seal on the face of one of the drillable inner sleeves. A very large force is created by surface-induced pressure differentials in the magnitude of 1,000 to $2,0001 \mathrm{~b} / \mathrm{in} .2$ operating on the crosssection of the piston created by plugs and sleeves, out to the seal against the collar housing inside diameter. In 9-5/8-in. casing, for example, assuming a collar inside diameter of 9.5 in., 1,000 1b/in.2 pressure would exert an opening or closing force of $1,000 \times 90.25$ in. ${ }^{2}=90,250 \mathrm{lb}$. Thus, if seals and plugs are properly applied, it is difficult to conceive of a heat-created interference or friction that could prevent sleeve movement under such forces -- yet such problems apparently exist.

Mechanical stage tools or port collars are also available. One device is actuated by vertical movement of automatically releasing, heavy-duty steel fingers which catch shoulders on the sliding sleeve. Another system utilized on shallower wells for special application operates with rotary motion. Both systems are illustrated schematically in Figure 2-5. 


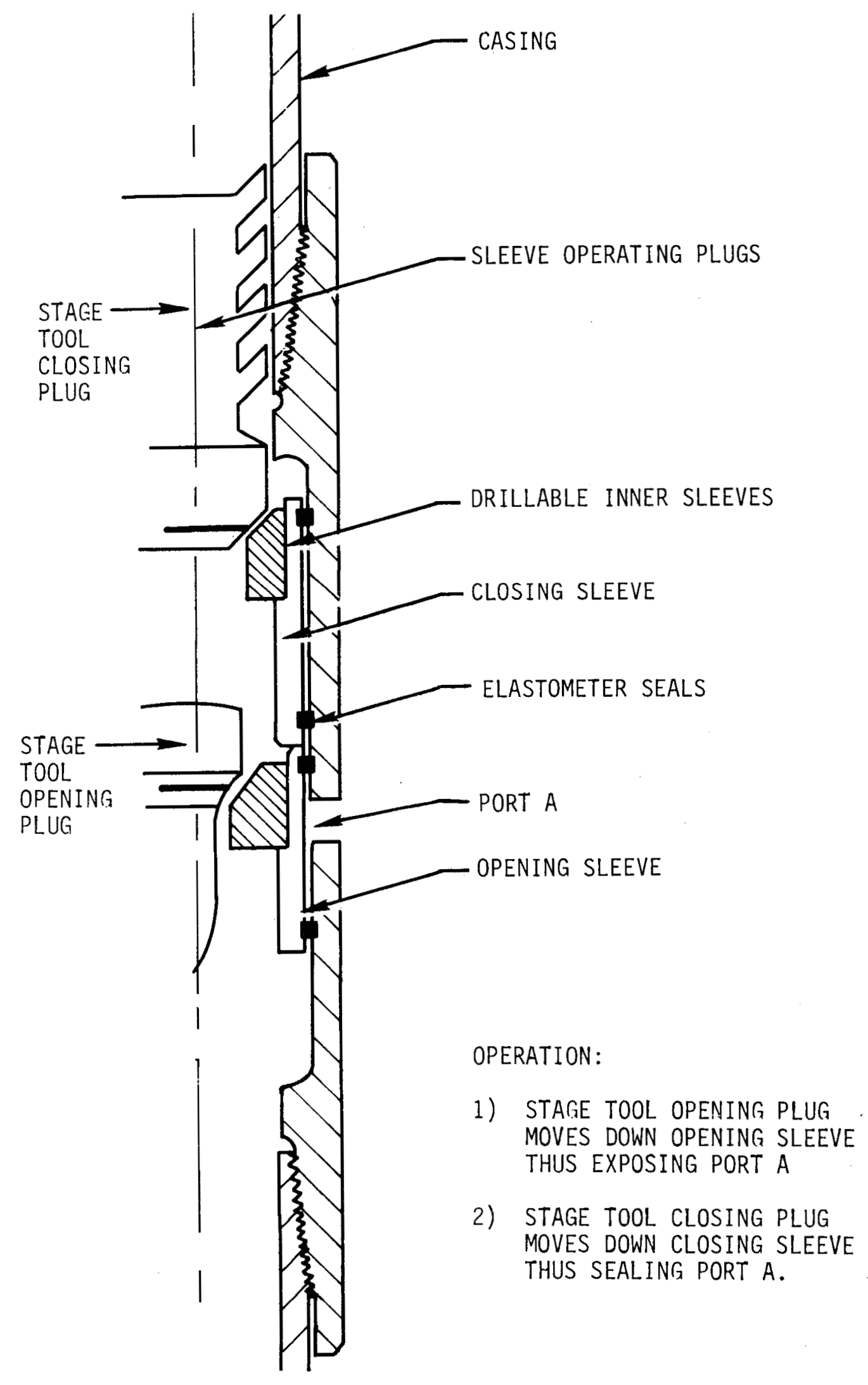

FIGURE 2-4. - Schematic of stage tool operation. 


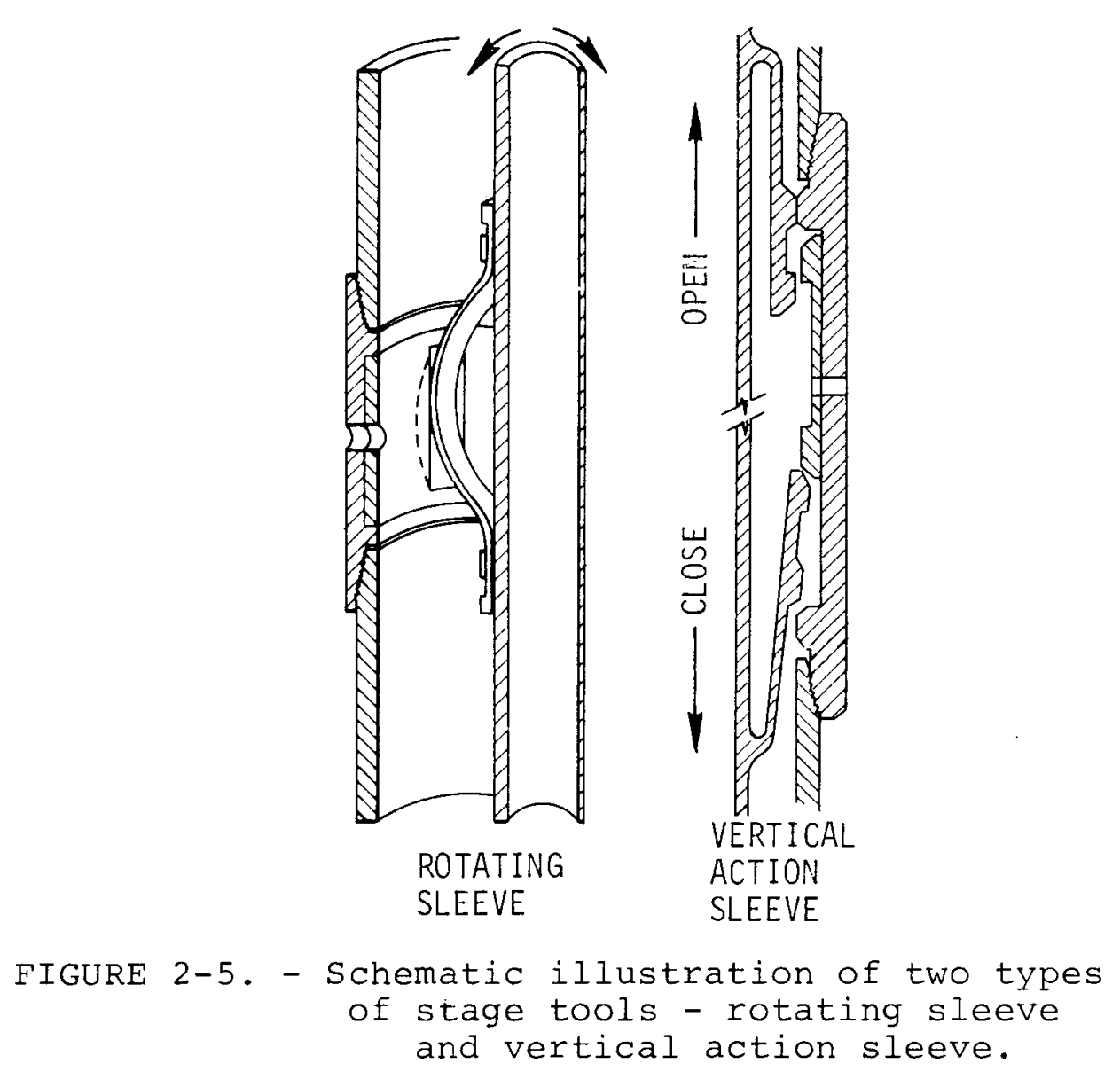

In geothermal wells, plug-operated stage collars are equipped with drillable cast iron inner sleeves in place of aluminum, which tends to become too ductile at high temperatures. One operator also utilizes a 90 Durometer Fluorocarbon elastomer for seals. It is believed that such modifications are typically all that is done to adapt standard tools to geothermal conditions.

Possible failure modes of plug-operated tools in severe downhole geothermal conditions are:

a. Elastomer seal seizure due to vulcanizing by heat effects

b. Differential expansion of sleeves and housings due to reactions of dissimilar metals, that is, cast iron versus steel, to temperature changes

c. Differential expansion of sleeves and housings due to temperature differentials inside and outside the casing (rising mud on the outside would be warmer than downward-moving cement slurry on the inside, by perhaps $100^{\circ}$ to $200^{\circ} \mathrm{F}$ ) 
d. Thermal stresses such as strong axial compression in the casing string being transferred to the collar and deforming the housing to bind or seize moving sleeves

e. Breakage of cast iron drillable sleeves due to heat, pressure and change in clearance between sleeves (where more than one stage tool is utilized, inserts added to the lower tools make plug seats successively smaller so that plugs can pass upper tools and land in the lower tools)

f. Inability of plugs to effect a pressure-tight seal on the operating sleeves.

of these failure modes, the most obvious, and possibly the least likely, is seizure due to elastomer vulcanization to metal. opening and closing forces are simply too great to conceive of an elastomer preventing movement. However, failure of an elastomer seal to prevent pressure loss or bypass due to a combination of property change by heat, and a change in sleeve clearances, may adversely affect certain tools. An example situation follows.

Differential expansion of sleeves and housing is probable under circulating conditions downhole. The resulting effect would be speculative until tested, but it is believed that seal performance could be adversely affected. As noted in Figure 2-6 (from Cain, et al., 1966, as shown in Reference 1) casing radius increase with temperature is significant. For example, by interpolation, 9-5/8-in. casing radius could increase about 0.007 to 0.008 in. with $200^{\circ} \mathrm{F}$ temperature change. This would increase shop-designed clearances of $0.005 \mathrm{in.} \mathrm{(typically)} \mathrm{to} \mathrm{nearly}$ 0.013 in. If differential pressure were exerted on the outer (hotter) component as well, an additional expansion of about 0.002 in. could occur, for each $1,000 \mathrm{lb} / \mathrm{in.}$.

Under conditions described in the above paragraph, an elastomeric o-ring that had been somewhat embrittled by prolonged exposure to heat may not be able to deform and adequately seal a gap that changes to as much as $0.015 \mathrm{in.}$. from an original $0.005 \mathrm{in}$. If this change were accompanied by a sudden pressure surge or shock, perhaps the seal may fail. However, note that in tools designed like those of Figure 2-5, even seal leakage would not likely prevent tool opening, as has been indicated to be the principal problem in Cerro Prieto.

\subsection{Sand Control Problems}

\subsubsection{Implied Problems}

Sand control is a potential problem for any geothermal producing or injection well which is completed in an unconsolidated formation. 

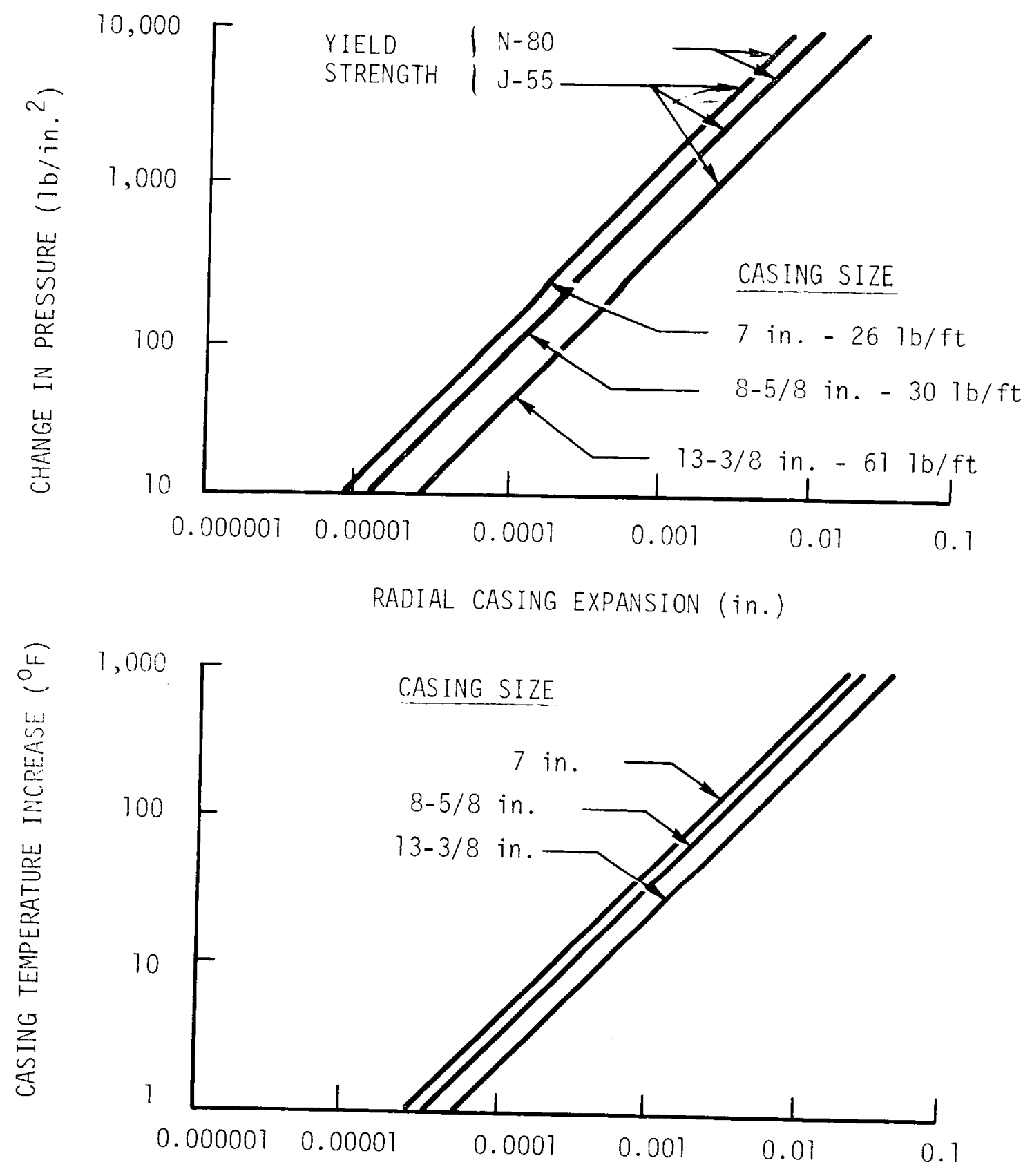

RADIAL CASING EXPANSION (in.)

FIGURE 2-6. - Increase in casing radius with pressure and temperature for several types and sizes of casing. 
Typical completions in geothermal production wells utilize long $(1,000$ to $4,000 \mathrm{ft})$ slotted liners which are generally designed for optimum hydraulics, without regard for sand control.

State-of-the-art sand control completion techniques developed by the petroleum and water well industries are not always adaptable to geothermal needs because of the high temperature, long completion intervals, and low reservoir pressure.

\subsubsection{Discussion}

Any production or injection well which is completed through an unconsolidated reservoir has the potential to flow sand into the wellbore. Sand inflow may also be accelerated during the life of the well as fluids pass through the formation. If sand influx becomes a major problem, it can result in production/ injection loss, damage to tubulars, and damage to pumping and surface treatment equipment.

Sand control problems are generally dealt with after the fact, that is, after several wells have been developed and it is determined that sand control is a problem for a particular area.

A review of several well files for the Imperial valley, California, area indicated operators are not trying to control sand even though there was evidence of sand fill in some wellbores (4). Typically, the production wells are completed over long intervals that may have a gross thickness of 1,000 to 4,000 f.t. Slotted liners (typically 7-in. to 9-5/8-in. OD) are hung in open hole, or blank liners are cemented and later perforated. Generally, only the permeable intervals which are used for production/injection are perforated or slotted. However, there are exceptions where the entire gross interval is completed with a slotted liner, exposing shales as well as sands.

Since the need for sand control is generally established after sand fill or sand production is noted, the operator should keep good records on the wells including accurate correlations of flow rates and sand production. Careful record keeping can establish need for sand control in new production/injection wells, and whether to implement sand control in existing wells by recompleting.

This decision is not as easy in geothermal wells as it is in petroleum or water wells because, as previously mentioned, completion intervals are often very long, and permeable zones may be scattered up and down the hole. In these instances, it would be very costly to set a screen/liner and attempt to gravel pack through several thousand feet of hole (or inside casing if undertaking a recompletion). Extensive treatment with chemical consolidation methods would be cost-prohibitive with currently available methods. 


\subsubsection{Identifying Problem Zones}

Obviously, all permeable zones in such large intervals may not be sand producers. Identifying which zones are unconsolidated and treating these (or eliminating them from communicating with the borehole by setting blank liner or cementing) would be advisable and more economical than treating the entire interval.

Some methods used to identify individual sand producing zones within a long interval are:
a. Measuring strength properties of cores in a soils laboratory
b. Correlating sand fill depth with produced flow rate profile
c. Setting straddle packers across permeable zones and measuring sand content in produced fluids.

Most of these methods are not yet proven for geothermal producers.

Logging methods may be used to derive mechanical rock properties for determining relative strength of formations, and whether they tend to produce sand $(\underline{5}, \underline{6})$. One advantage of this technique is that the entire interval of interest is measured in one operation. The Mechanical Properties Log ${ }^{\circledR}$ derives a "G Ratio" which is the ratio of the shear modulus of the rock to its bulk compressibility. This value is then compared to an empirical value of $G$ (generally derived for a specific region) to determine the zone's potential for sand production. The suite of logs which must be run to compute this log contains neutron, sonic, gamma ray, and resistivity logs. Schlumberger has tested all of these tools in $500^{\circ} \mathrm{F}$ environments so the data should be reliable for geothermal wells in this temperature range (ㄱ).

Most of the work with this log has been done in the United States Gulf coast region and it would be advisable to develop an emperical G-value for, say, the California area. Some operators probably have this suite of logs on existing wells, which could be used to compute the Mechanical Properties Log.

\subsubsection{Iimitations of Chemical Methods}

Once sand producing zones have been identified, sand control methods can be more economically undertaken.

Sand control in a problem well can be effected by two principal means:

\footnotetext{
*Mechanical Properties Log ${ }^{\circledR}$ is a registered trademark of Schlumberger.
} 
a. Mechanical methods which utilize screens, gravel packs, prepacks, etc.

b. Chemical methods which provide in situ consolidation by injecting or placing cementing agents or resins ( 1 ) .

Unless properly engineered, sand control measures may cause further production loss by reducing permeability. Some of these problems include introduction of fines during gravel packing, swelling of formation clays, and (for chemical methods) residual resins left in the formation from ineffective backwash or overflush.

Chemical sand control techniques developed for the petroleum industry are not useful in high-temperature geothermal wells with long intervals for the following reasons:

a. Cost is too high except in small applications.

b. It is difficult to inject effectively into all portions of large intervals as treatment material will take the path of least resistance (most permeable section).

c. Maximum recommended operating temperature for most proven techniques is 2000 to $3000 \mathrm{~F}$ (9).

One manufacturer which recently entered the market claims to have a material which is stable in a $500{ }^{\circ}$ environment (10). This material, called super Sand, requires no flushes or liquid resins as it is a dry, resin-coated sand which sets with heat once placed in the formation. It merits further investigation as it is easily placed with water carrying fluids.

Any chemical consolidation material would require additional testing to check for stability in a geothermal environment, that is, effects of temperature, brine, changes in $\mathrm{pH}$, etc. Choice of workover and stimulation fluids would also need careful attention, as some of these resins could become unstable in the presence of treating chemicals.

Small, individual zones which were identified as possible candidates for sand control could be individually treated with these consolidating materials if they were determined to be stable in a geothermal environment. Schemes might include setting open-hole straddle packers and pre-packing before setting a liner, or setting, cementing and perforating a liner and then washing and packing the perforations which needed sand control.

\subsubsection{Proposed Gravel Packing Concept}

The more conventional techniques for sand control, setting a screen/liner and gravel packing, also present problems particularly if it were necessary to gravel pack the entire gross interval. 
However, individual sand-producing zones, if identified, could likely be either gravel packed or shut off, depending on production or injection capacity. A scheme for this type of completion is shown in Figure 2-7; Figure 2-8 shows detail of a placement procedure which uses commercially available tools and hardware $(11,12)$. In the scenario in Figure 2-7, the production liner is hung-off in the drilling liner in the usual manner. Slots are used opposite consolidated sands, screen is used opposite unconsolidated sands, and blank pipe is set opposite sloughing shales or nonproductive sands. These intervals would be identified by previously mentioned mechanical properties and correlation logs. This scheme could also be used for recompletions which use a through casing (or liner) gravel pack.

\subsubsection{Completion Fluid/Gravel Pack Material}

Other considerations for a successful gravel pack in a geothermal production/injection well are needed for effective completion fluids for carrying the gravel, and the gravel pack material itself. Both of these items can be adversely affected by very high temperatures and accelerated chemical reactions.

In a conventional gravel pack for a petroleum or water well, gravel is usually placed in the annulus between the screen and borehole or casing with a clean, water-based fluid. This fluid may be modified by adding various materials to prevent fluid loss, increase the gravel-carrying capacity, and maintain hole stability in open hole or in unconsolidated sand behind perforations. Common fluid loss agents are calcium carbonate, ground resins or rock salt. Viscosifying agents are generally starch or CMC, and polymers such as HEC or XC. Viscosifiers can be used in fresh water or brine and may have to be broken by other chemicals such as acid. All of these commonly used viscosifying materials break down rapidly at temperatures in excess of 2500 to $3000^{\circ}$, which would be the lowest temperatures encountered in geothermal reservoirs.

Another problem with using fluids modified to maximum carrying capacity is that of weight. Most geothermal reservoirs have a low-pressure gradient and are drilled with mud weights of about $9 \mathrm{lb} / \mathrm{gal}$, or even less. Because of this, it might not be possible to work in high-temperature, low-pressure wells with fluids which carry large amounts of gravel, without losing circulation.

Contact was made with several companies which provide completion fluids service and completion materials for the drilling industry. Most service companies suggest using fresh water if weight is a problem, that is, gravel pack without the viscosity. Lightweight brine would have to be used if the fluid must be made compatible with shales or clay minerals $(13,14)$. One manufacturer said it might be possible to use polyacrylamates or polyacrylamides which can be mixed with fresh water for a hightemperature system $\left(400^{\circ}\right.$ to $\left.500^{\circ} \mathrm{F}\right)$. This material would require 



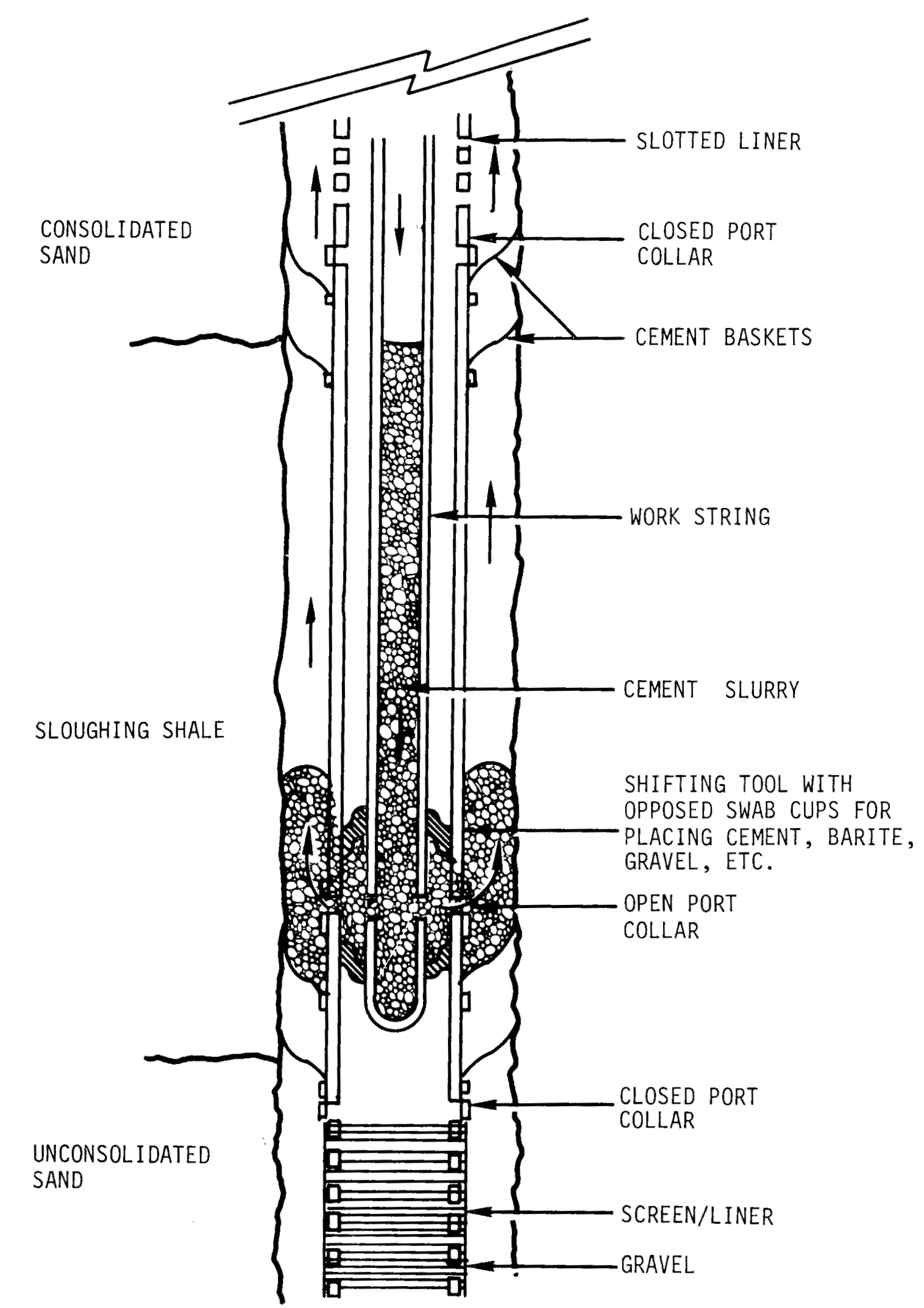

FIGURE 2-8. - Detail for selective gravel and cement placement behind liner in geothermal well. 
a breaking agent; and extensive testing in a simulated environment would be necessary before it could be recommended (15). Another possibility is to simply monitor returns and add conventional polymer as the system degrades. However, this procedure could be costly and difficult to monitor, thereby making the gravel packing operation inefficient.

Further investigation of cross-linking of polymers could be valuable research providing it does not duplicate industry efforts already underway to develop a much-needed high-temperature polymer.

Conventional gravel materials are basically silica minerals with varying amounts of impurities. This material will degrade at high temperatures as evidenced by the high dissolved silica content in geothermal wells which produce extremely hot water $\left(>500^{\circ} \mathrm{F}\right)$. The $\mathrm{pH}$ of the formation fluid also has a marked effect on the amount of silica dissolved from the formation (16).

A cursory review of granular materials available in common mesh sizes was made. Some of these materials are used by the petroleum industry for gravel packing and fracturing (sands, glass beads, bauxite), and some by the filtering and abrasives industry (carborundum, corundum, silicon carbide, garnet). Garnet is a natural silicate which is available in common mesh sizes and is used in the food industry for filtering hot (usually low $\mathrm{pH})$ liquids (17, 18). This material might be further investigated for use as an alternative gravel packing material in a geothermal well. In commercial grade, it is roughly four times the cost of sand. Some of the abrasives (corundum, carborundum) are also possible candidates for testing but they are very expensive and might be cost-prohibitive in large quantities. Any of these materials should be thoroughly tested before being used in a geothermal well gravel pack completion.

\subsubsection{Recommendations}

State-of-the-art sand control is probably not directly applicable to geothermal producing wells because of the hightemperature, aqueous environments which attack and/or degrade both chemical consolidation and gravel pack materials. And it is impractical to effectively place gravel packs in very long producing intervals. Success with sand control in injection wells would depend on existing temperature conditions at time of installation, which may or may not be as severe as those in producing wells, and potential reactions with injected fluids with varying chemical properties.

Suggested items for further study and development are:

a. Develop data which would make mechanical-propertiestype logs reliable in detecting zones which require sand control. Joint efforts involving operators and a reputable logging firm are recommended as use of logs from active areas would be required.

$$
2-22
$$


b. Investigate materials which could be used in sand control -- either for gravel packs or chemical consolidation techniques. Such a study would review existing materials, discuss engineering problems, undertake laboratory tests for stability in geothermal environments, and address costs and availability. CTC is aware of one such study which was done in connection with industrial disposal wells (19). Gravel pack dissolution in a harsh, downhole environment was studied, and alternate materials were addressed, and ultimately used in the field.

c. Define requirements and potential applications for an economical, lightweight completion fluid for gravel packing and related operations. One manufacturer contacted during this study expressed interest in further development in this area (15).

d. Further develop the concept for selective treatment of individual zones in a long gross interval as suggested in Figures 2-7 and 2-8. Economics of this completion versus commonly used slotted liners (without sand control) could be presented in detail. All hardware is currently available through vendors and service companies. Operator cooperation could be solicited to make economic and technical proposals credible.

e. Define requirements for slotted or wire-wrapped screens to be used in producing and injection well gravel packs. Cost-effectiveness and reliability of stainless steel wire-wrapped screens in hot, chloride brines would be compared to possible limitations of lower-cost, saw-cut slotted liners. Representative sand distribution analyses would be required. The study would evaluate productivity impairment through packs and screens considering special needs of systems with high rate flows at low drawdown pressures.

\subsection{Effective Inhibitor Placement}

\subsubsection{Implied Problem}

Scaling and corrosion of metal goods in geothermal wells which produce gas cut, highly mineralized fluids are operations problems. These two phenomena can impair and restrict flow from wells, and, if uncontrolled, can lead to costly repair and workovers.

There are currently many schemes under investigation and/or testing to remove scale after it occurs (20). However, costeffectiveness of these methods has not been proven. Corrosion, 
of course, causes permanent damage. One alternative is prevention through use of inhibiting chemical treatment -- unfortunately, reliable methods for inhibition treatment have not been developed.

Even if an effective high-temperature inhibitor were available, some operators may not be able to justify its cost under present economics. However, corrosion damage and loss of production added to cost of periodic scale removal may leave the operator with hard choices such as whether to maintain inhibition or face expensive well recompletion or replacement. For scaling, some cost comparisons for inhibition and removal are available for geothermal wells (20).

As reported in the following discussion, this study investigates neither limitations nor the state of the art in chemical inhibitor development. Assuming such materials may soon become available, the technology of inhibitor placement downhole in a producing well is discussed and concepts for protecting a well containing large-diameter downhole pumps are offered.

\subsubsection{Discussion}

Depending on well geometry, pressure/temperature relationships and fluid chemistry within the well system, scaling and corrosion can occur in a variety of forms and locations in nearly any wet steam or hot water well from the wellbore/formation face through the wellhead and surface equipment. If the wells are artificially lifted, pumping equipment may also be damaged. The problem is generally not present in dry steam producing wells and it is less severe in liquid producing wells which have low mineral content. All reinjection wells are subject to corrosion and certain scaling problems depending on how waters are handed on surface.

Sweet corrosion (associated with $\mathrm{CO}_{2}$ in solution) is the most common type of corrosion process in geothermal wells. Sour corrosion ( $\mathrm{H}_{2} \mathrm{~S}$ present) is not common in the United States. oxygen corrosion, obviously, would be most prevalent in surface facilities and reinjection wells. At present, the potential for corrosion in producers is recognized but little positive action is being taken as failure rates and related technical requirements have not been identified.

Scaling can occur at any point in the well system where precipitation of the minerals in solution can occur. Generally, scaling occurs around pressure changes in the system, that is, across perforations, screen/liner openings, liner tops, pump intakes, wellhead equipment, etc. Also, in a flowing well, pressure loss at shallow depth causes flashing, further gas evolution and cooling which severely imbalance the hot liquid, and cause solids to precipitate. Common precipitates found in wells are calcium carbonate (lime) and calcium/magnesium sulfate (gyp) (2l). 
Several methods are available or have been proposed for scale and corrosion inhibition in low-moderate temperature wells. These inhibition schemes include two categories, chemical and electrical. Chemical inhibitors are available in solid and liquid form and are the most widely used type. Electrical devices are relatively new for application in deep wells and are not currently used (20). Because many different types of chemical inhibitors exist for different types of scaling and corrosion, they are the most logical choice, and the most widely used by the geothermal industry. Other schemes or thermal recovery methods which prevent scaling by preventing flashing and gas evolution in the wellbore are being investigated by some geothermal operators as alternative development programs.

This report will discuss downhole placement of chemical inhibitors which currently exist and are temperature-stable. The marketplace contains inhibitors which reportedly are stable in the $350^{\circ}$ to $500^{\circ} \mathrm{F}$ range $(22-26)$. The field reliability of inhibitors in the $500^{\circ}$ to $60 \overline{00}$ range is not proven, and new developments for this temperature range are needed for the hotter geothermal wells. A parallel study could be useful in developing inhibitors with long life stability -- in the range of months.

In any case, it is assumed that, to be effective, the inhibitor must be placed in the bottom of the well in such a manner that it is carried through the entire producing well system by the produced fluid. Often, corrosion and scaling may occur on tubulars at the formation face. Two basic methods exist for so placing chemicals:

a. Solid material can be injected into formation fractures to be produced back over an extended time period, or

b. Liquid or solid chemicals can be pumped or otherwise transported to the bottomhole location through separate conduits or another type of container device, to effect continuous or periodic treatment.

Several service companies currently offer in situ stimulation treatment schemes which inject or squeeze inhibitor solutions or solids into fractures during completion and workover operations $(24,25)$. The amount of inhibitor placed into the formation fractures (natural or induced) is based on assumed fracture geometry and time between successive stimulations, that is, during workovers or when the well is down for other reasons. These calculations are somewhat imprecise. When the presence of inhibitor is no longer detected in the produced fluid, it is time to initiate another treatment. These methods are being investigated as part of the DOE stimulation contract administered by Republic Geothermal, Inc. 
The in situ placement method is only effective for long time periods when the inhibitor is placed away from the wellbore into fractures. Matrix treatments which could be undertaken with a much simpler operation are not long-lived as the injected inhibitor is produced back rapidly.

Although this method is acceptable and proven, it is not operationally flexible in that it is a "one-shot" process. Once the treatment is planned, and pumped away, the operator must accept the results until it is convenient to shut in the well and again. stimulate. Additionally, the inhibitor must be stable for long time periods as residence in the formation must be for months (or treatments are not economical). Finally, treatment should make use of existing or initiated fractures or it may not be cost-effective unless done as part of a major fracturecreating stimulation.

Continuous downhole placement of inhibitors in the wellbore during operation would not be difficult in a flowing system with open casing to surface. Two packaged systems currently available in the marketplace represent methods which could be used to continuously, or intermittently inject liquid inhibitors at any depth in the well $(27,28)$. Such a scheme is shown in Figure 2-9. One supplier claims the system can be used to monitor bottomhole pressure (BHP) and intermittently inject inhibitors (28). This technique is currently being evaluated by the petroleum industry and, reportedly, by the geothermal industry. However, little information was available at the time this report was prepared $(\underline{28}-\underline{30})$.

These systems are basically chemical injection systems which make use of a low-volume, high-pressure metering pump on the surface. The desired amount of inhibitor is injected through a small-diameter (0.25- to l.00-in.) tubing run to the desired depth in the well.

For BHP monitoring, the tube (usually 0.094-in. OD) is equipped with a pressure chamber and sinker bar, and run to the desired depth; the system then is charged with an inert gas such as helium or nitrogen. Liquid inhibitor may be introduced down the same tubing without modification. When it is desired to return to BHP monitoring, the system would be purged with a cleaner, and the gas would be recharged into the tubing. Obviously, rate of inhibitor to be injected, length and size of injection tubing, and pumping pressure are interrelated and would have to be carefully designed.

Because of the limit in axial strength of the thin-wall tubings associated with BHP monitoring, the amount of scale which might build up on the outside of the tubing when there are no inhibitors in the well should be considered. This weight 


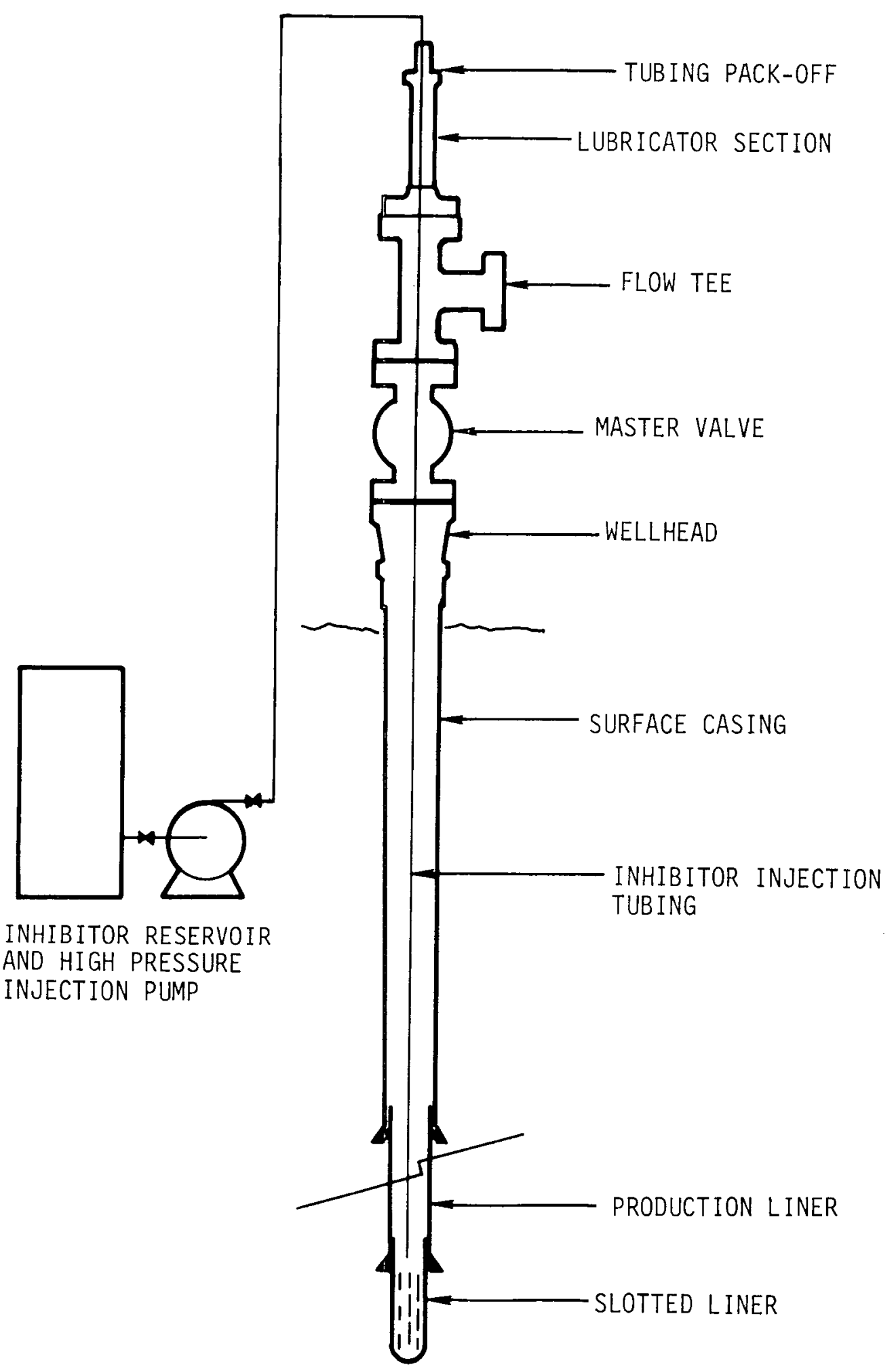

FIGURE 2-9. - Inhibitor injection in flowing well. 
should be added to the hanging weight of the assembly when designing the system. The small-diameter tubings (0.094-in. OD) typically have a tensile strength of $600 \mathrm{lb}$. Considering the limitations, dual use of tubing for accurate BHP monitoring and effective inhibitor placement should be carefully evaluated before purchase and installation.

Placement of inhibitors in a well which requires artificial lift becomes more complex because of clearances by the pump bowls, Figure 2-10. Reportedly, operators have tried running small-diameter tubing strings between the pump bowl and casing annulus for BHP monitoring. These installations have not been successful as the tubing is usually damaged in areas of minimal clearance, that is, at the floor when passing casing and tubing through spider bowls or BOPs, and at the pump bowls. Typically, an operator will run a maximum volume production pump which necessitates large-size impellers and bowls. For example, 12-in. bowls in 13-3/8-in., $61 \mathrm{lb} / \mathrm{ft}$ casing may provide only $1 / 4$ in. annular clearance. The most commonly used pumps in geothermal wells are line shaft vertical turbine pumps as shown in Figure 2-10. Submersible electric pumps are relatively new to the geothermal industry and are used on a limited basis (31).

Another method of inhibitor placement which has had some success in pumped wells is that of circulating inhibitors in the pump's line shaft lubricant (32). This method would at least be partially effective in protecting the pump. However, casing and liner below the pumped section are still exposed to scaling and corrosion.

Figure 2-11 shows a scheme whereby newly arilled wells could be equipped with an external tubing string which is "plumbed into" the surface string. This would allow access for inhibitor injection systems (and wireline tools if the tubing were large enough) below expected pump landing depths.

Obviously, the drilling program would have to provide for a bit size which would give necessary annular clearance to run the desired size tubing in conjunction with the surface casing. For example, 13-3/8-in. casing (14.375-in. OD collars) run in 17-1/2-in. hole would allow for an external tubing size of 3/4-in. nominal, flush joint (1.05-in. OD) or mild external upset (1.327-in. OD). Larger tubing which would pass logging tools, that is, 2-3/8-in. or larger, would require large hole size and may not be feasible. One argument for smaller-size tubings would be their flexibility, which would be necessary near the surface and at the point where the tubing is attached to the casing. 


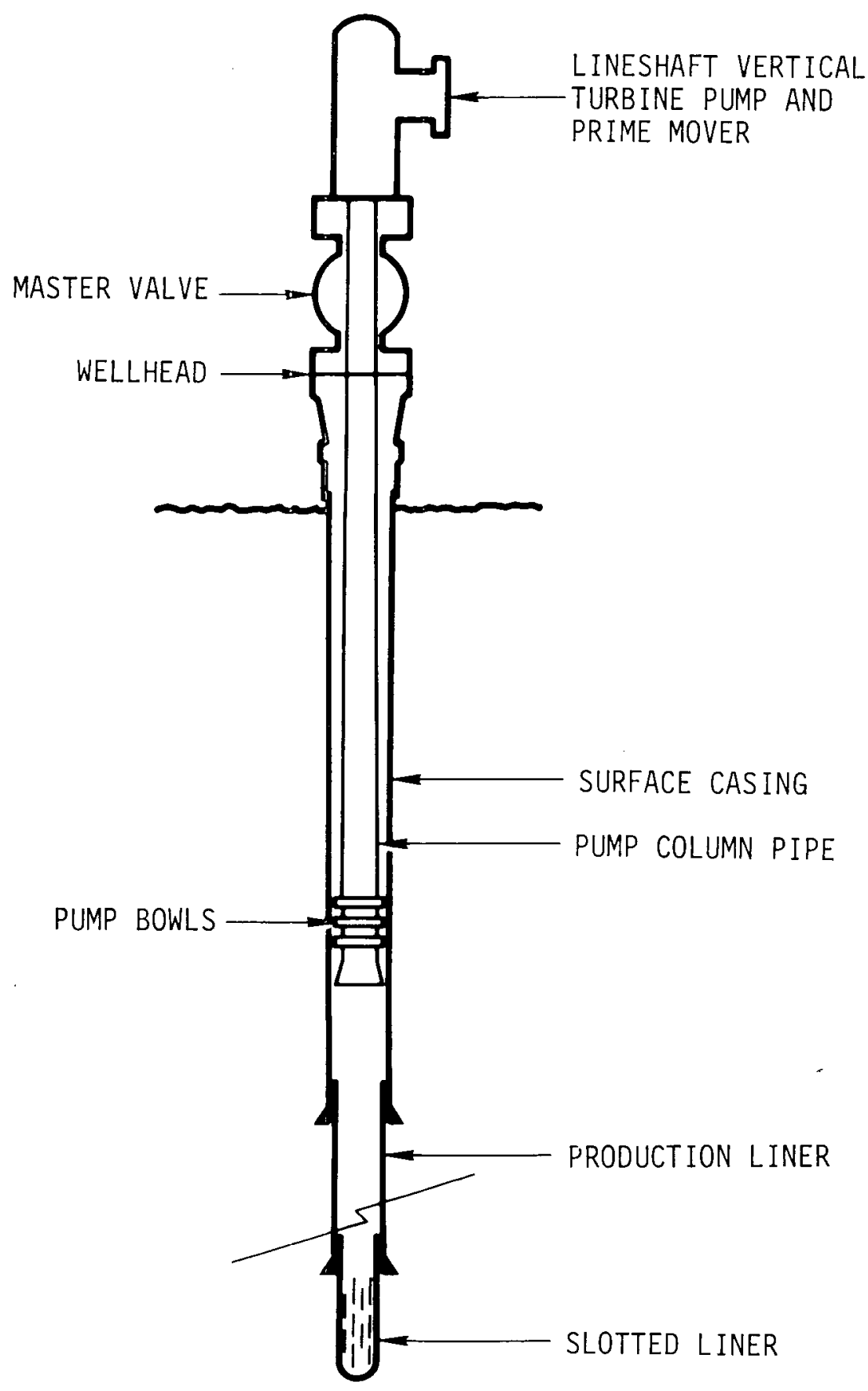

FIGURE 2-10. - Pumped production well.

$$
2-29
$$




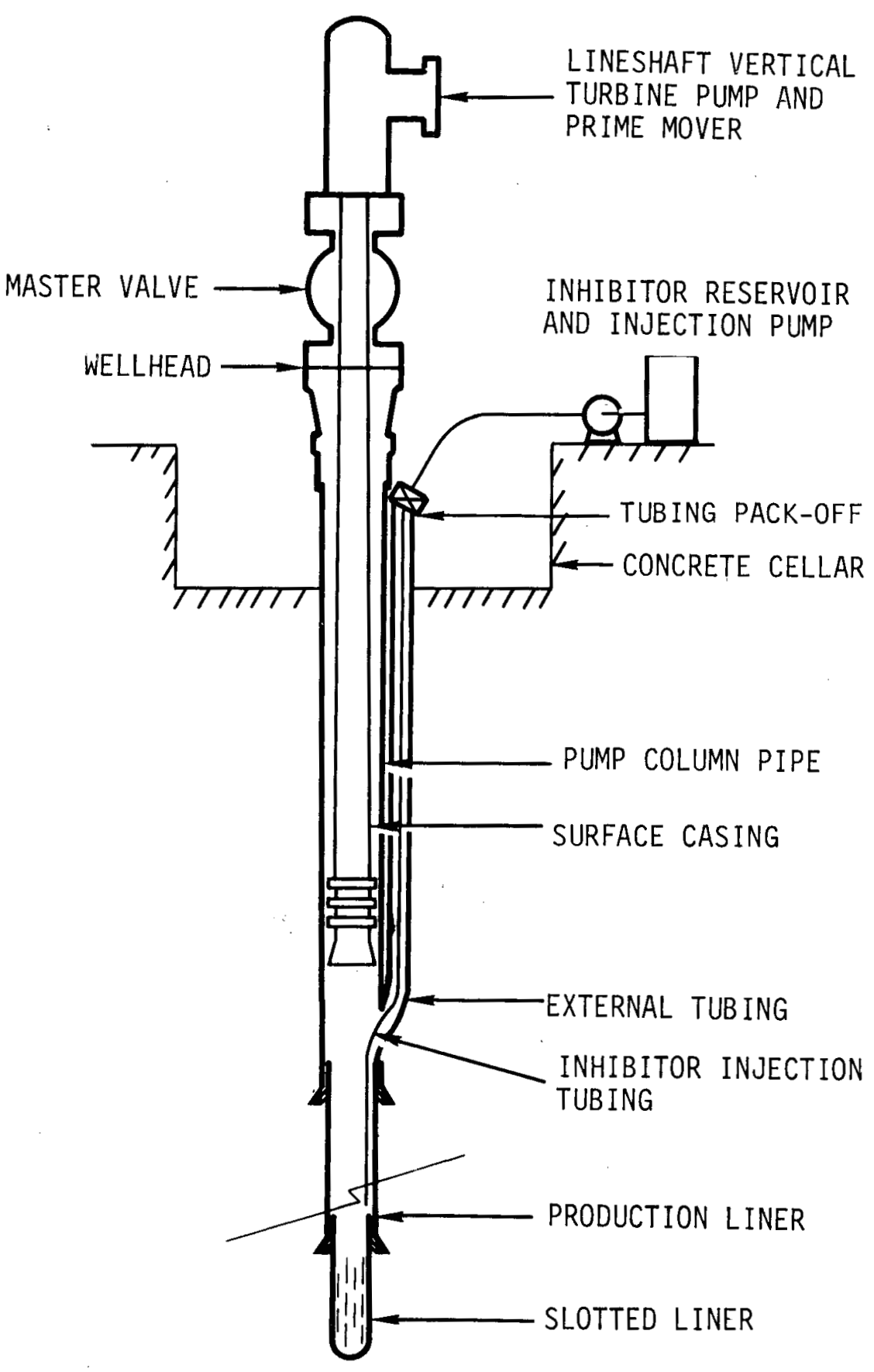

FIGURE 2-11. - Scheme for inhibitor placement in a pumped well. 
The tubing inlet into the casing string could be "muleshoed" or tapered sufficiently to provide a gradual transition through the entrance if it were welded directly to the casing. Attaching the tubing could also be accomplished through a weldneck type fitting or a specially-made short casing nipple with a threaded bushing.

Figure 2-12 shows how the two strings could simultaneously be run in the hole and later cemented. Conversations with a reputable casing crew service company indicate this procedure is practical with existing casing handling tools $(33,34)$. As the tubing is run with the casing, it could be attached by common, hand-held banding machines.

With regard to cementing operations, if the casing is cemented using an inner work string, that is, stab-in shoe, no precautions would be necessary to prevent the external string from being filled with cement. If the plug method is useful, a temporary bridge plug could be located in the tubing at the point where the tubing is connected to the casing. It should be flush with the casing inside diameter. Presumably, it could be made from wood, plastic, paraffin, etc., and later knocked out with a slick-line sinker bar or pumped out with surface pressure.

The minimum depth at which the external tubing is fixed to the casing would be some convenient depth below the maximum expected setting of the pump suction. Small, flexible injection tubing such as that previously mentioned for use in flowing wells then could be run inside the external tubing and set at any depth in well. This would require running the injection tubing to the well bottom if maximum coverage of the inhibitor were desired.

This external tubing concept merits further investigation as it would be relatively simple to install and would provide necessary access to the well bottom for running inhibitor systems and possibly some wireline tools.

\subsubsection{Recommendations}

Investigations conducted for this study indicate that continuous inhibitor injection through small-diameter tubing is feasible for flowing geothermal wells, using commercially available systems. Further, inhibitor placement within the formation as part of a hydraulic fracturing stimulation attempt appears to be a viable concept providing a long-life inhibitor is made available and volumes required are economical for infrequent applications.

However, use of small-diameter tubing to place inhibitors in the bottom of wells containing artificial lift pumps is not practical with existing completion configurations. One concept 


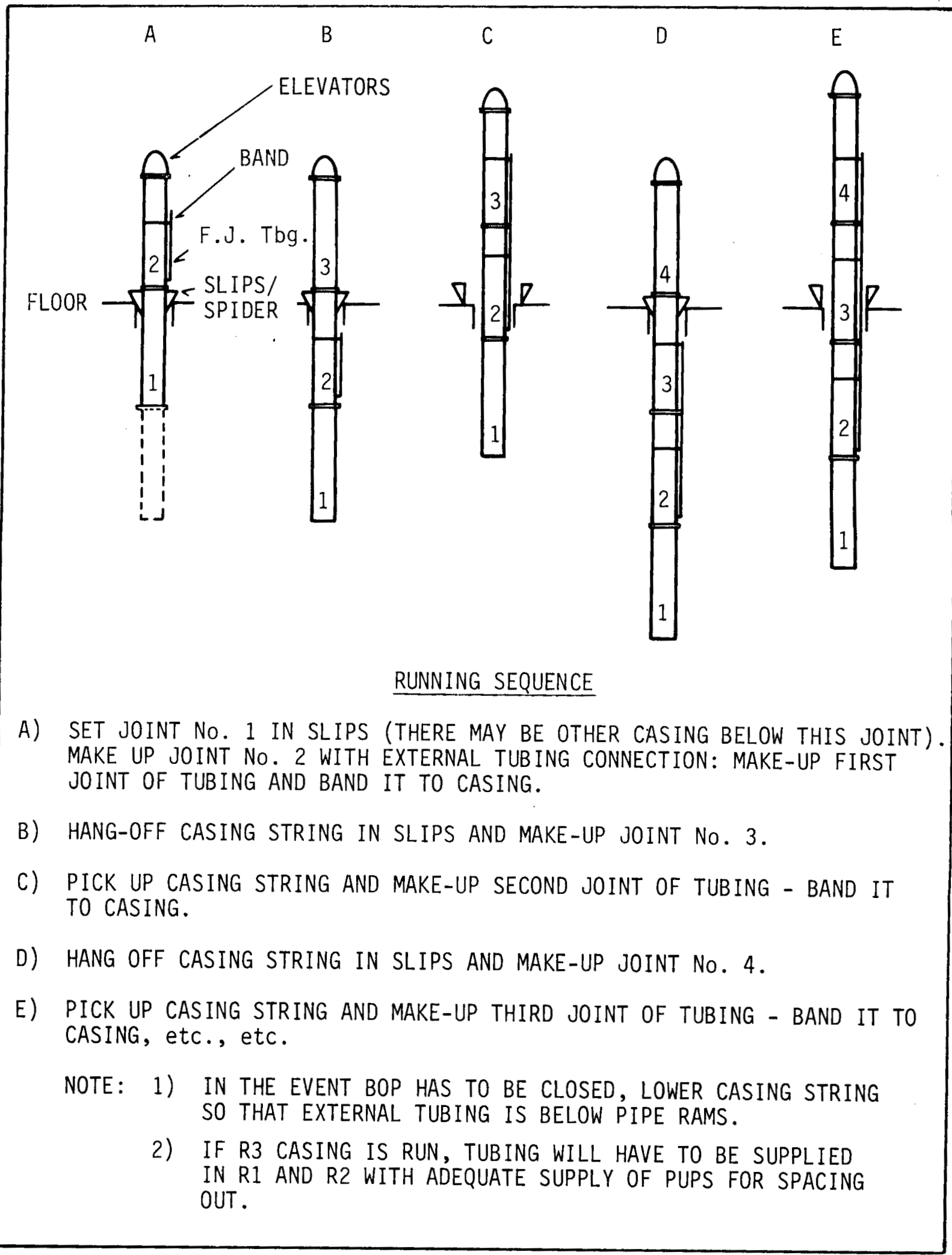

FIGURE 2-12. - Casing running sequence with external tubing string. 
for modifying existing completions to make available inhibitor injection viable in such wells is the addition of an external tubing string as illustrated in this report. It is recommended that implementation of this new concept be further studied by evaluating the following engineering problems:

a. Determine most feasible tubing size for typical casing programs and investigate available tubing string sizes, connections, strengths, etc.

b. Determine most suitable type of connection for plumbing tubing into casing. Consider welding, machined fittings, stresses at bending points, etc.

c. Investigate problems and failure modes which might be caused by external tubing, such as effect on cement jobs, remedial action if external tubing develops a leak, temporary plugging to permit conventional casing cementing, thermal stresses on the composite string.

d. Estimate installation costs and effect on well economics.

\subsection{Slotted Liner Iimitations - Zonal Isolation}

2.4.1 Implied Problem No. 1: Lack of Effective Zonal Isolation

At present, the annular space between blank sections of slotted liner and the open hole cannot be sealed so as to form an effective barrier to fluid migration behind the pipe, between active production or injection zones. Such communication between zones prevents selective testing and/or treating of individual zones and profile control, in injection-production schemes, that would allow optimum recovery of in situ heat. Because of this limitation, slotted liners are not an equivalent alternative to effective cementing and perforating.

\subsubsection{Discussion}

Geothermal wells may be completed in one of three ways:

a. Open hole

b. With slotted or otherwise perforated, uncemented Iiner

c. With solidly cemented and jet perforated liner or casing. 
Only the most competent of formations allow completion in the open hole; example areas are The Geysers and Roosevelt Hot Springs, where fractured volcanic rocks do not cave or fall into the borehole with fluid inflow, and where larger rock particles can be cleaned from the fractures to prevent damaging particle flow with production.

In other areas exemplified by the Baca Ranch development and deep wells in the Salton sea and Cerro Prieto, formations are "hard." However, they are primarily cemented and fractured sands or "welded" volcanic dusts that contain intervals that are or can become unstable and slough or flow into the borehole with hot water movement and pressure drawdown. There, the high permeability of an open hole is achieved by installing a protective liner that limits borehole wall movement yet offers little flow restriction through numerous, very large, slots.

Another type of well, of which there are many to be drilled, is that in the moderate temperature $\left(340^{\circ}\right.$ to $400^{\circ} \mathrm{F}$, perhaps) sand-shale sediments of several Imperial Valley fields. Two examples are East Mesa and Heber area formations, to depths of perhaps $7000 \mathrm{ft}$.

In these areas, prolific and thick sand bodies feature a broad range of properties varying from relatively large-grained, unconsolidated sands at shallow depths, to well-cemented metamorphosed fine-grained sands and silts at either greater depths or within hotter zones above heat domes (35).

In wells described above, operators would prefer to cement casings or liners solidly through the gross sand-shale interval so that individual sands could be selectively perforated, as determined by electronic logs previously run in the mud-cooled hole. Advantages of cemented pipe include:

a. The cement sheath firmly supports the borehole wall against movement, caving or sloughing.

b. Cement between perforations acts as a barrier to fluid flow between perforations, certainly between perforated intervals.

c. Blank spaces between intervals can be used as bridge plug and packer seats so as to selectively test or treat.

d. Zones can be blanked off temporarily or permanently to modify production or injection profiles to achieve proper reservoir control. 
A well such as that shown in Figure 2-13 would be drilled with a modern temperature-resistant mud such as sepiolite. The liner would be assembled after running open hole logs, incorporating any devices that would be required to affect the interzonal seals. After running the liner and installing the seals, the slotted sections would be washed to remove drilling mud.

General criteria required of any interzonal sealing material or mechanism are:

a. The seal should positively block any movement of fluids into or out of the formation behind the blank pipe under all possible pressure differential conditions, either positive (during injection, treating or stimulation), or negative (during highest rate drawdown).

b. The seal should be permanent under long-term exposure to maximum downhole temperatures.

c. Sealing materials or devices must be noncorrosive and otherwise nondamaging to the liner.

d. Sealing systems should be effective under and compatible with thermal stresses induced into the liner by expected future operations.

e. Systems should be cost-effective and simple enough to be assembled quickly in the field and installed using state-of-the-art service company capability, with minimum technical supervision.

It is not known exactly what attempts are currently made to isolate slotted liner sections. Some operators indicate that it is not uncommon to locate cement bridging baskets near the bottom of blank sections to catch sloughing shales. Such fill would not be an effective pressure barrier unless a significant portion of the shale caved in--in which case the liner may be subjected to strong external loading which may lead to collapse failure. It is also possible that the "dead space" in the liner-borehole annulus may be susceptible to external corrosion in contact with packed shales.

Some technology from thermally stimulated production areas may be relevant. Shell oil Company of Venezuela (now Maraven S.A.) published a report of several relevant concepts used in improving gravel-packed wells subject to up to $600^{\circ} \mathrm{F}$ steam injection (37). The relevant items mentioned are thermal expansion joints for the liner, liner top pack-off assemblies with graphite and metal rings, special mandrels and port collars for selective injection, and a system for injecting a plugging agent into a shale section between sand zones. 


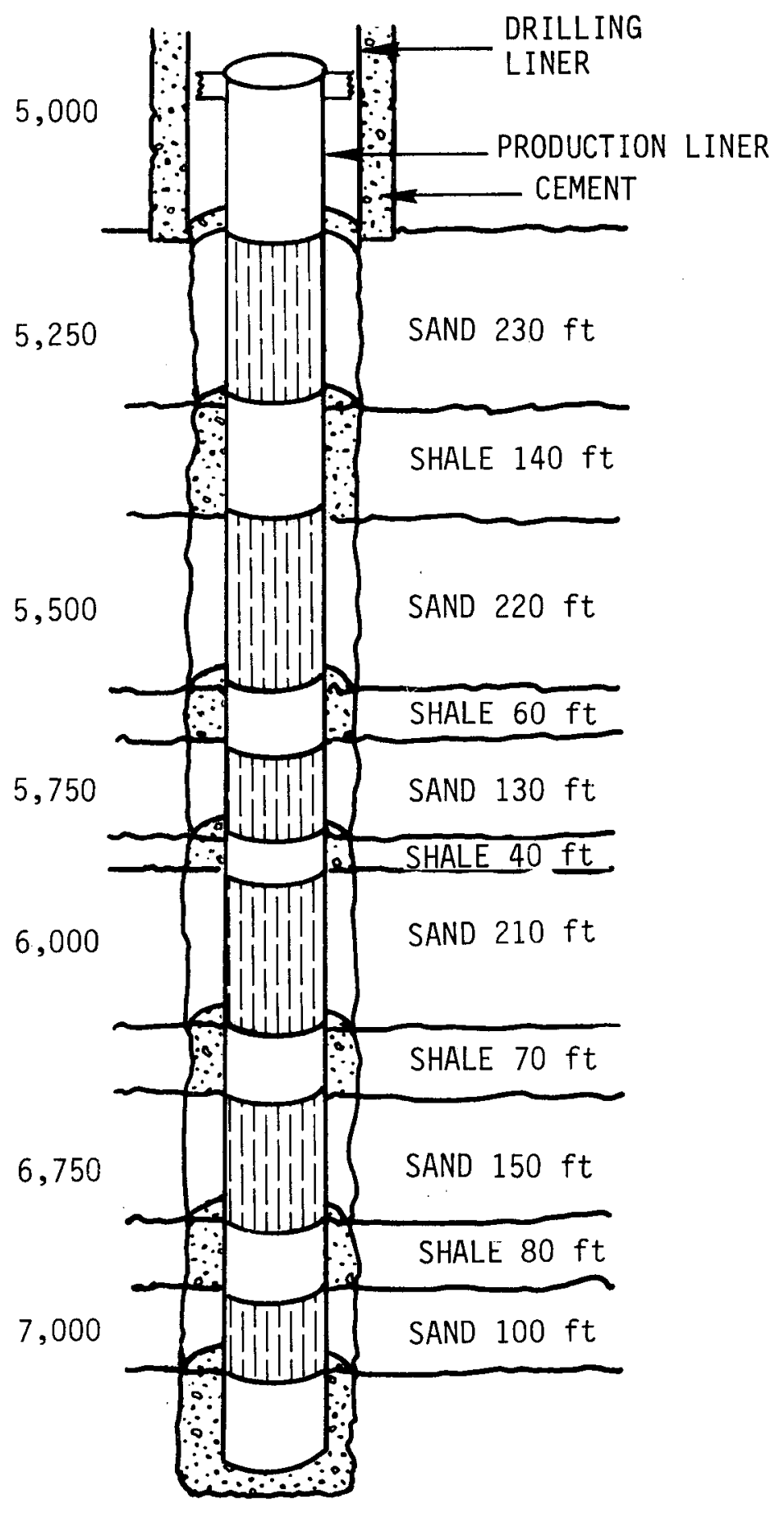

FIGURE 2-13. Concept of zonal isolation between sand intervals in an Imperial Valley production of injection well. 
Also, the THUMS operating company of Long Beach, $C A$, is utilizing a relatively inexpensive cementing scheme to circulate cement through a shale zone between sands. The original concept as described in World Oil's Sand Control Handbook (38) utilized two inflatable packers, between which cement was circulated into a lower port and out an upper port. The modified system, which is noted in the $B \& W$, Incorporated catalog (Composite Catalog, Vol. 1, 1980-81 Edition), utilizes two cementing basket sets (one looking up and one looking down) in place of the inflatable packers (Figure 2-14). This system would not be directly applicable to a deep and initially hot well, but the isolation concept is representative of geothermal well needs.

\subsubsection{Recommendations}

At this time, it is the opinion of CTC that geothermal operators would have mixed feelings about the priority of developing this new technology. Because liners can be, and are being, installed in very simple and trouble-free configurations with little risk, reliable systems would have to be offered, despite agreement from engineers that they are needed for optimum reservoir management.

Consequently, it is suggested that the next action be a detailed feasibility study that would: define expected costs and risks for alternative concepts; and judge industry acceptance based on its review of detailed proposals.

Specific tasks may include the following:

a. Define potential applications considering published development schedules for Baca Ranch, East Mesa and Heber areas initially, also potential reinjection well systems for these areas plus developments in hotter areas such as the Salton Sea-Brawley-Niland area.

b. Describe potentially applicable systems in enough detail to assess their technical and operational practicality.

c. Estimate direct and related operator cost to install such systems.

d. Present above study to management personnel of active geothermal operating companies and assess future program support. 


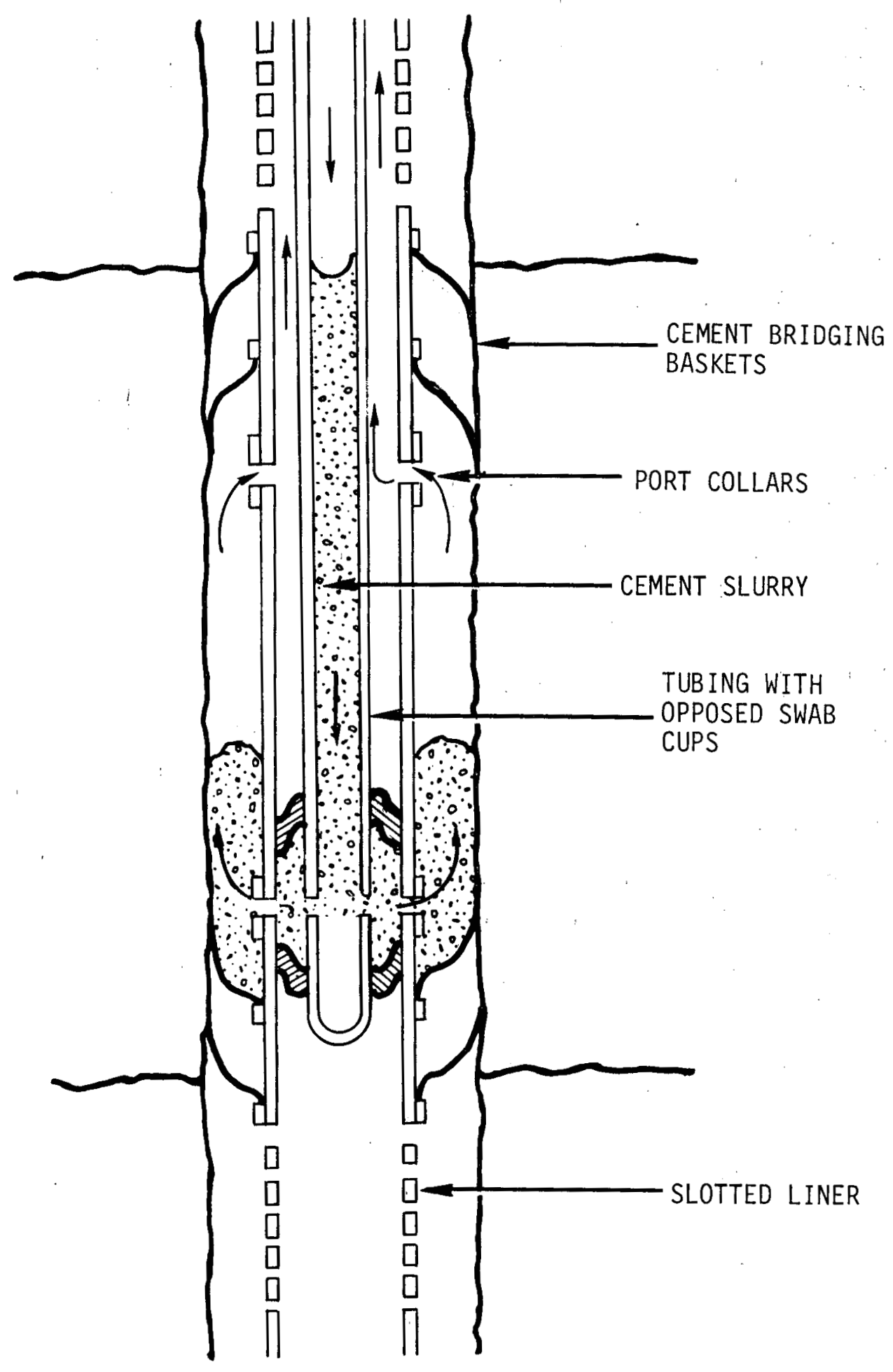

FIGURE 2-14. - Concept of circulating cement slurry through a thick shale interval between slotted liner sections. 
e. Assess potential system market outside the United States geothermal activity area and within the larger oil and gas industry, as a consideration for private manufacturer interest.

f. Define development needs, including research time schedules, new component or materials requirements and possible DOE/Sandia participation, if private industry development is unacceptable or impractical.

\subsection{Slotted Liner Limitations - Liner Washing}

\subsubsection{Implied Problem No. 2: Limitations of Liner Washing Methods}

Geothermal producing wells are sensitive to restrictions and plugging within the borehole because reservoir pressures nearly equal the hydrostatic head of water, and wells are unable to generate high inflow velocities through exposed pore spaces to purge heavy drilling mud lodged between slotted liners and the formation face. The mud, and any shale or rock plugging in the liner slots, is normally removed by jetting and flushing action of water which is pumped out through the slots from a work string and circulated to the surface. It is speculated that this operation is inefficient in very long producing sections because:

a. Heavy, heat-thickened drilling mud and filter cakes on the formation face may not be removed.

b. Some slots may remain plugged with rock debris.

c. Hydrostatic pressure may drive mud-contaminated, less viscous wash fluids into the porous rocks and fractures and cause permeability damage well beyond the formation face.

d. Jet impingement may fluidize sands on the formation face and cause particle invasion beyond previous filter cake depth.

\subsubsection{Discussion}

The objective of a slotted liner completion is to have the advantages of an open hole from the standpoint of unrestricted. permeability exposure, and still prevent the problem of hole caving by the support of steel casing. Ideally, through sands, there would be an open annulus between the liner outside diameter and the borehole inside diameter and that annulus would be communicated to the liner inside diameter with, typically, 16 2-in.long $\times 0.25$-in.-wide slots per foot. 
Through shale zones between sands, the liner may be blank (unslotted) or not. In either case, shale swelling or caving around the liner would not be a problem unless it fell into the sand zone below; some operators install bridging baskets below long shale zones to keep lower sands clean.

\subsubsection{Mud Removal Mechanisms}

In the ideal sandstone-slotted liner configurations, it is difficult to visualize drilling mud presence as a restriction to hot water inflow as the very large slot area should allow water inflow to dilute and flush any nonpenetrating filter cake and residual mud into the liner inside diameter for removal. Some may even argue that the optimum initial action, after mud is displaced from the liner inside diameter, would be highest possible inflow from the formation, to wash loose the residual mud in the liner-borehole annulus. One system for doing this, is to inject nitrogen into the casing through a continuous tubing string to quickly lighten. the column and reduce hydrostatic head at the formation face. This procedure has been labeled "well shocking" by service companies.

However, formation clean-up by natural fluid flow may not appear so feasible when a very detailed look is taken at mechanisms operating at the formation face. For geothermal, hot water wells producing from matrix permeability and not fractures, and with long producing intervals, these types of factors should be considered:

a. Inflow velocity over a unit of area of the borehole face is small, despite high total well flow. For example, in a well with an 8-3/4-in. borehole with 1,000 . net feet of permeable sand, flowing 50,000 bbl of water per day, the flow rate through 1 in. 2 of the exposed formation face is only $0.52 \mathrm{tbsp} / \mathrm{min}$. At 16 slots per foot, the rate through any one slot is only 1.46 cups/min.

b. The ideal open Iiner-borehole annulus may not exist due to borehole instability. Studies have not defined the behavior of hot water bearing sands under differential pressure. It is conceivable that the borehole may shrink in response to sudden stress relief in the magnitude of several hundred pounds. While the magnitude of hole diameter change may be small, clearance between 7-in. liner and 8-3/4-in. hole is only 0.875 in. If a weak sand actually filled the annular space, the exposed permeability would be reduced to that of the slot area--this would significantly reduce the system's flow capacity. 
c. Thick mud cakes may fill the annular space. Logs through high permeability sands in the Imperial Valley indicate conventional muds can build thick filter cakes. The example of Figure 2-15 illustrates a caliper log through sands and shales in which a diameter reduction of 1 to $2 \mathrm{in}$. is indicated across zones with high sand content.

d. Liners are likely not centralized in the open hole. It is not common practice to use centralizers on long slotted liners. Therefore, the string can be expected to follow the lower side of the hole, even in moderately deviated wellbores. Collared pipe is normally used, as flush joint casing is considered too weak for geothermal liners. The collars would provide some standoff, but they would also drag mud cake and cuttings off the lower side and pack them into slots, so the benefit may be questionable. The eccentric position in the borehole will surely add to annular restriction on one side of the liner and reduce the clean-out efficiency of fluid inflow.

Thus, the above-noted factors indicate that inflowing fluid energy may be quite weak and not enough to "flush" mud and drilling cuttings that may be tightly packed into the liner annulus. With some zones so blocked, it is likely that production does not come uniformly from all exposed permeability. Short sections with very high productivity indices (flow rate per pound-per-square-inch drawdown) will contribute most of the flow and long intervals may remain plugged.

\subsubsection{Washing Concepts}

The principle of the circulating wash method is that water can be pumped out through the liner slots at a high velocity and the resulting "jets" will penetrate and dislodge mud and mud cake from the borehole wall where the cake is being held onto the wall by differential pressure, Figure 2-16. By pumping the water out of the work string between downward and upward looking swab-type packer cups, the fluid velocity can be controlled by varying packer cup distance and thus varying the number of slots through which the fluid is pumped, Figure 2-17. When the pumped fluid enters the annular area and dislodges the mud, the fluid must change directions and move upward to above the top packer. There it will likely reenter the liner through cleaned slots and be circulated to surface. A portion of the flow will move up the Iiner-borehole annulus at low velocity as the total open volume between the borehole and the work string outside diameter is large. 


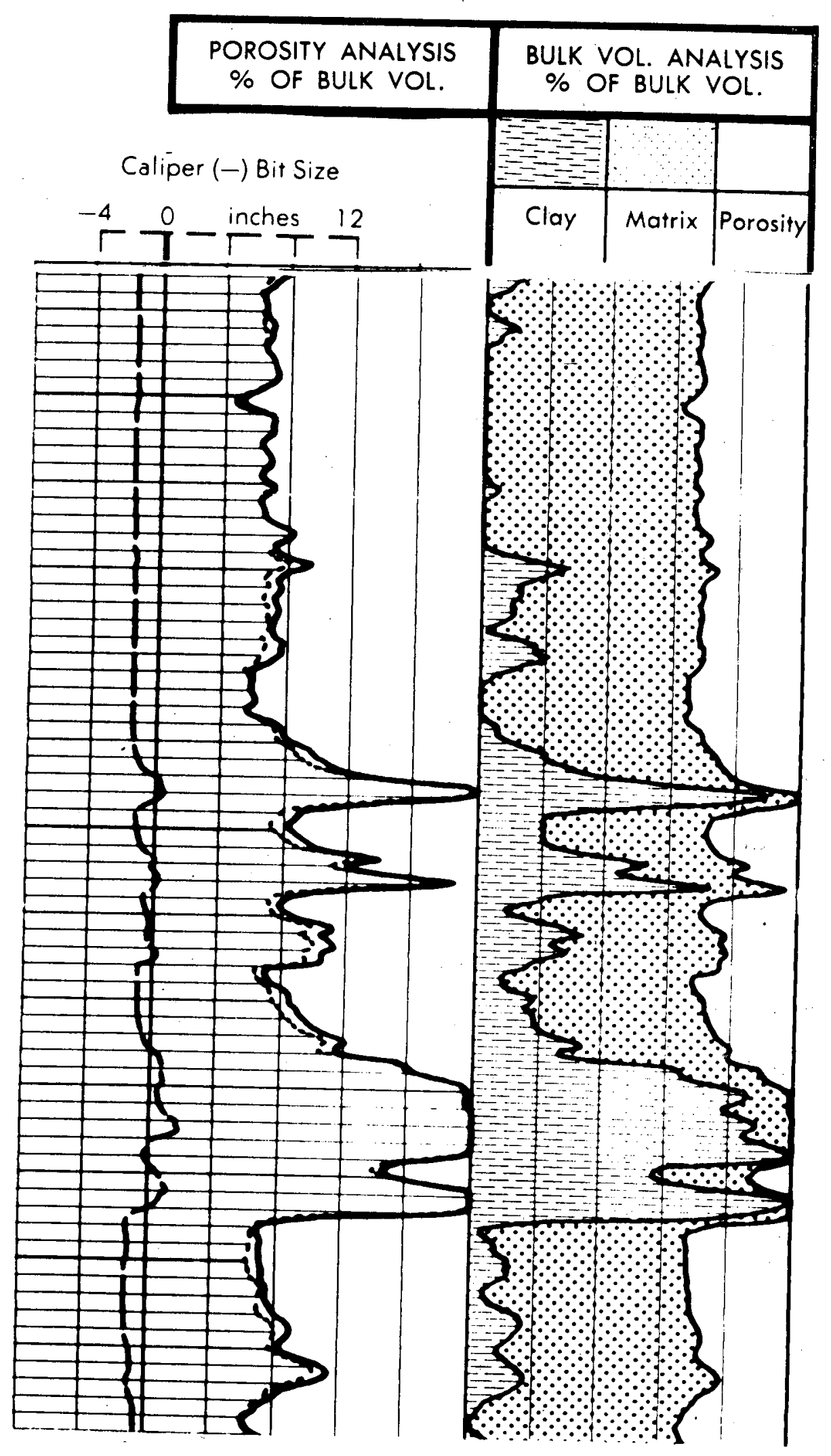

FIGURE 2-15. - Example Saraband log section from East Mesa well showing thick mud cake over porous sand intervals. 


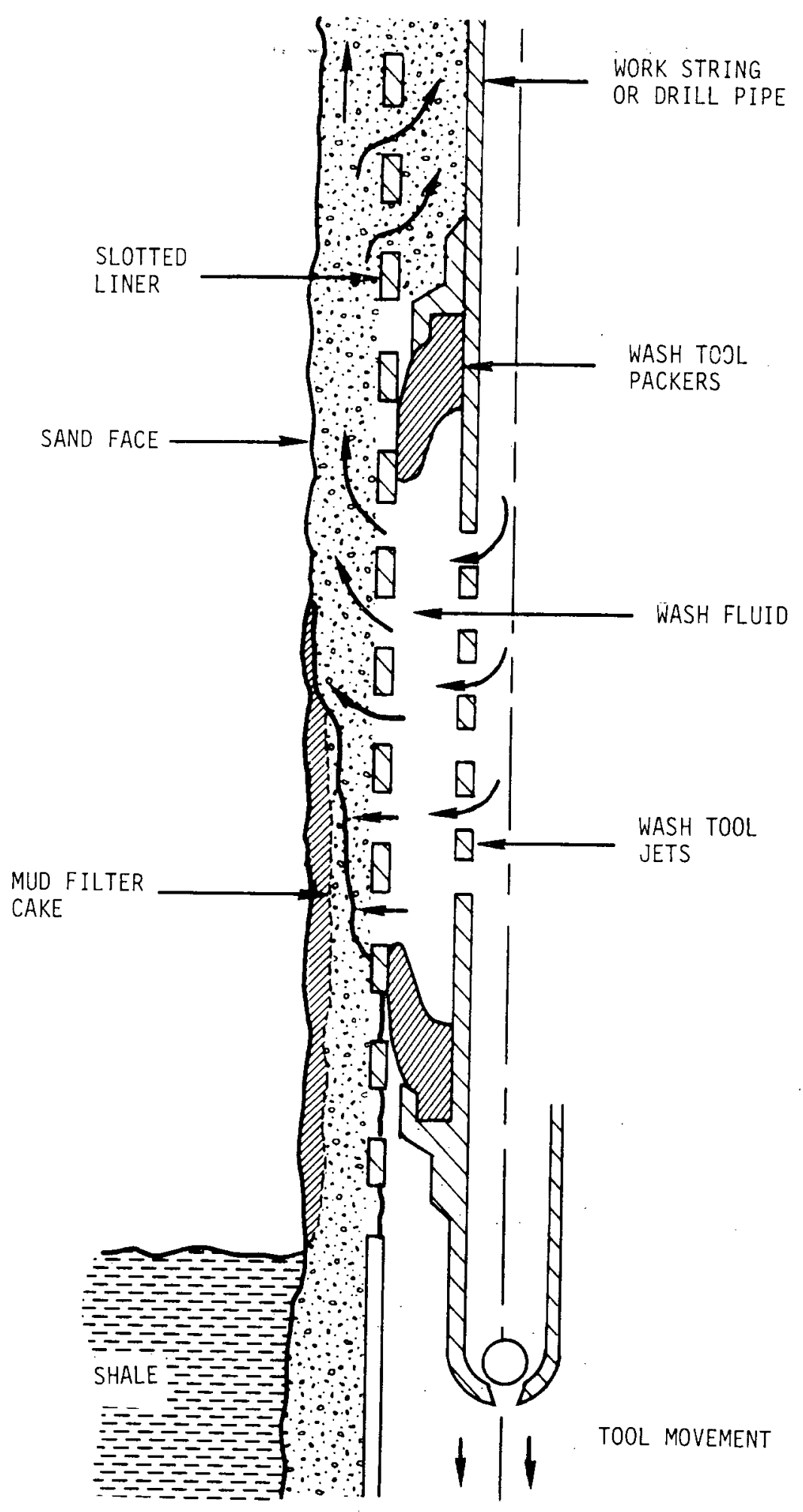

FIGURE 2-16. Schematic of wash fluid removing mud behind slotted liner. 


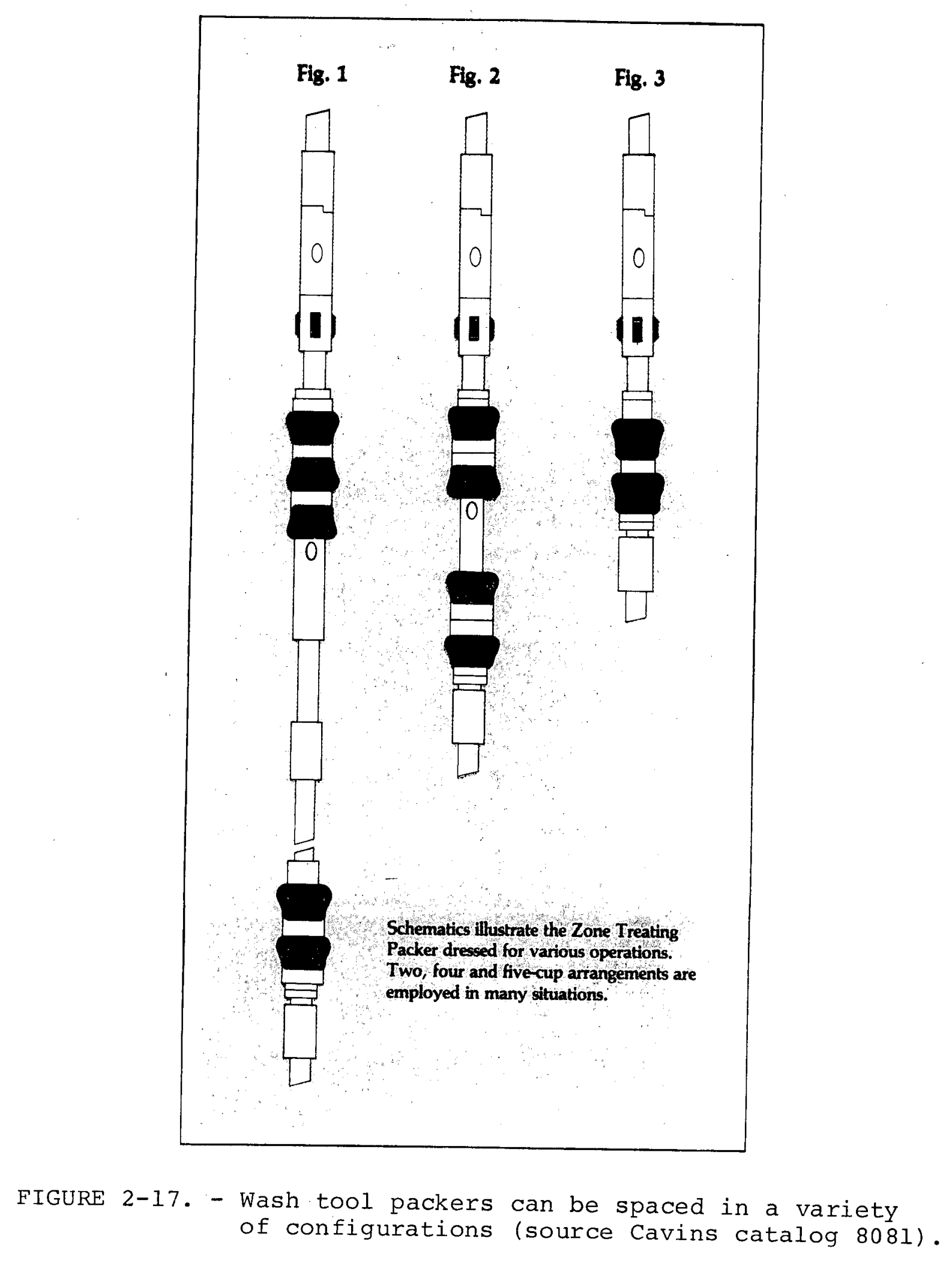


Various commercial tools are available as shown in Figures 2-18 and 2-19. Nearly all tools have features that allow downward washing while lowering the tool through the liner initially, to displace mud from inside the liner. And ports can be opened to permit circulation above the top packer to remove sand fill, or to allow fluid bypass while running in the hole. Some operators own and maintain wash tools.

A variation of the above procedure is used to wash perforations. In this procedure, the objective is to reduce packer spacing so that fluid positively jets through the perforation and not only fluidizes the sand around the perforation cavity but actually washes out a concentric cavity around the cement between perforations such that a large volume is created behind pipe for gravel packing, Figure 2-20. This process is a very inexact operation and few industry engineers would claim to know what happens. Unfortunately, no physical models have been tested and described to help understand the phenomenon.

Thus, for geothermal liner washers, the problem may be how to perform the washing so that the velocity through the slot is adequate to create a dislodging and flushing effect beyond and between slots and at the same time protect the freshly exposed and vulnerable near-surface sand face from damage from the wash water itself--and the high volume of contaminating particles it then contains. If fluid velocity is too high, it may impinge upon the formation face and penetrate to a significant distance by fluidizing the grains and/or displacing the surface fluids by pressure.

The wash water should be chemically compatible with the formation, because considerable volumes will be lost to the cleaned sands above the washer as the water will be cooler and more dense and will be quite easily injected into the high capacity sands. If very little fluid is lost during washing, it is rather positive proof that the wash fluid has indeed plugged the surface of the formation so that fluids cannot be injected.

In perforation washing, one alternative solution to fluid loss to washed zones is to wash with a fluid loss additive in the brine-based wash fluid. This usually requires building viscosity with a polymer and then adding finely-ground calcium carbonate particles that plate out on the exposed permeability and create a thin, tough film that controls fluid loss. The film then is removed by reverse flow or an acid wash. This alternative is not likely to be feasible for geothermal wells due to the high cost of brine fluids, polymers and additives, and the difficulties associated with acidizing in hot holes. 


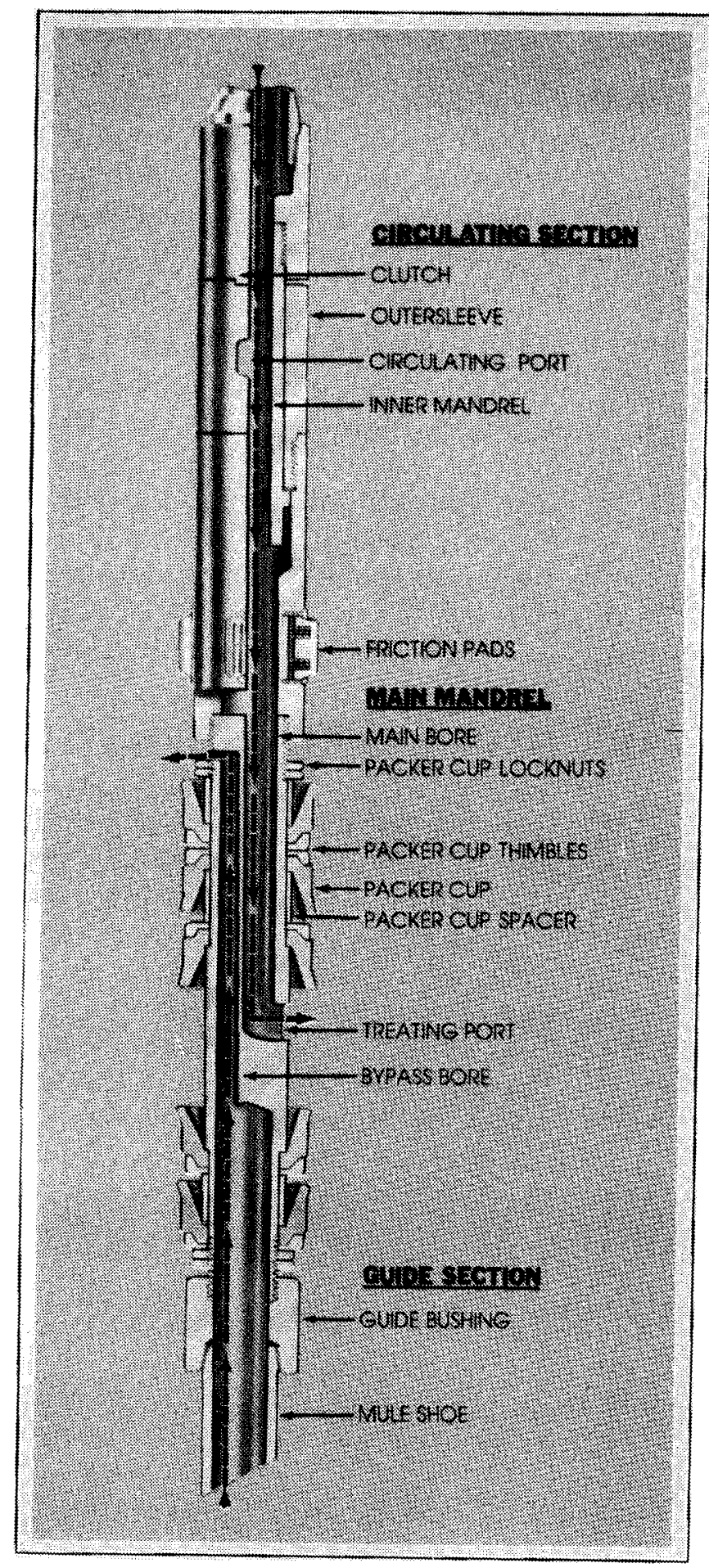

FIGURE 2-18. - Wash tool operations including bypass bore, treating port, and top circulating port. (source Cavins catalog 8081 ). 


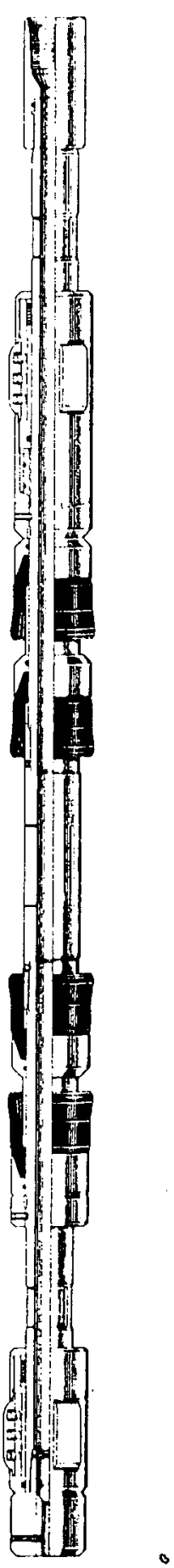

a) BROWN OIL TOOLS
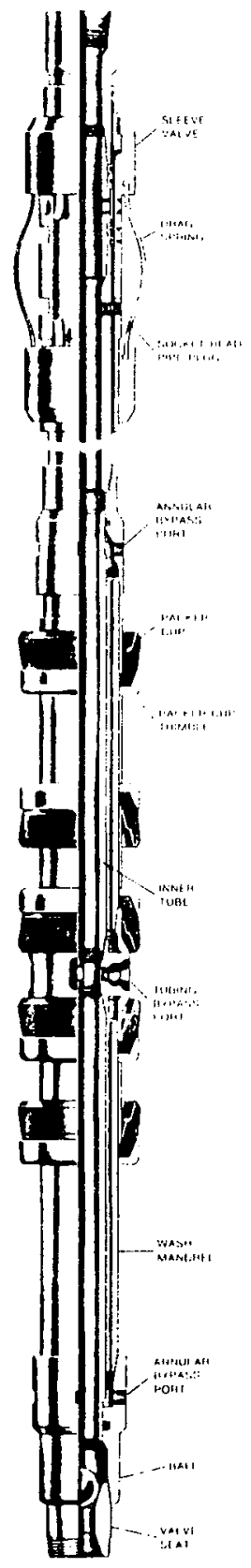

b) BAKER SAND CONTROL

FIGURE 2-19. - Example commercial wash tools. 

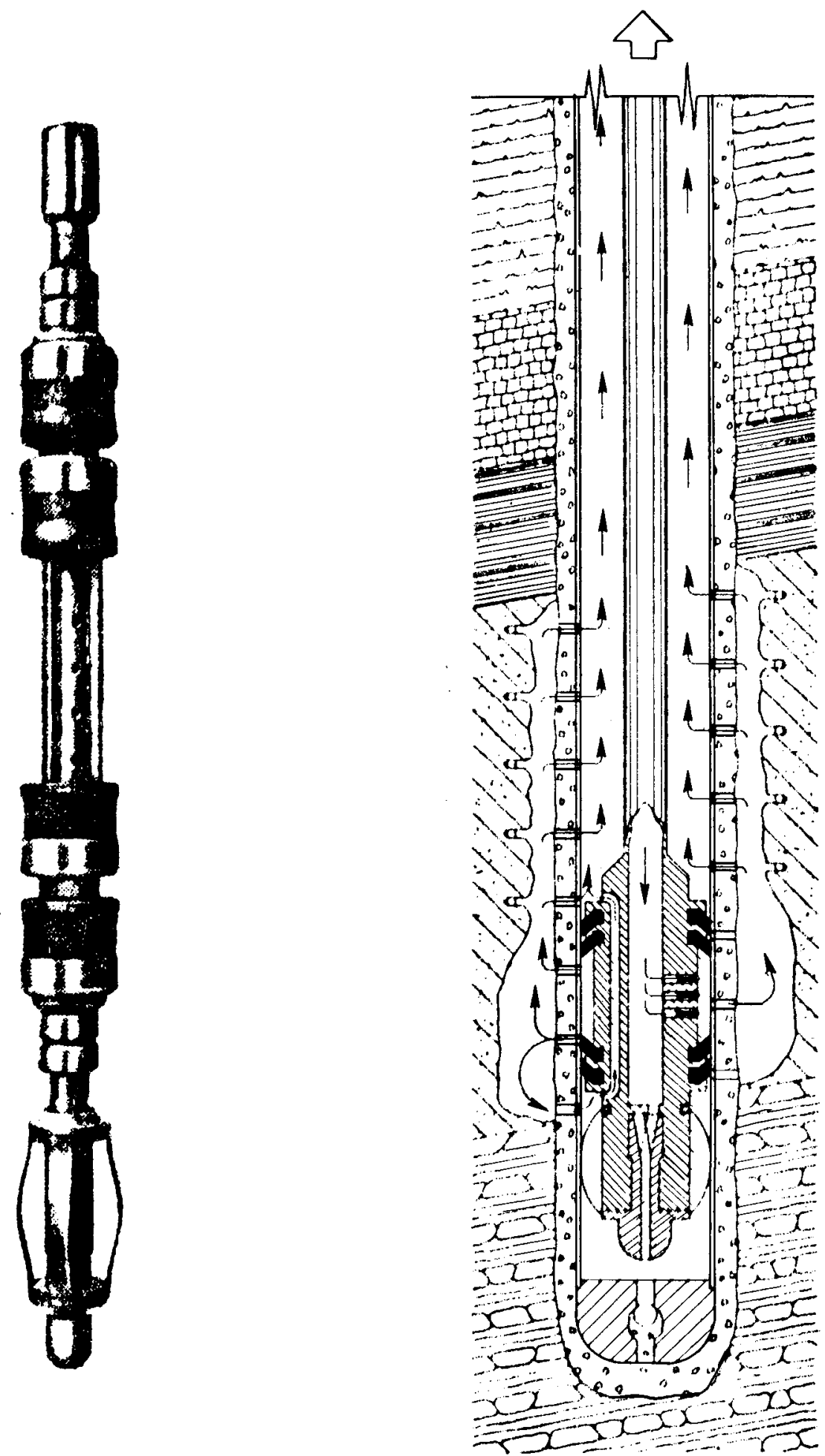

FIGURE 2-20. - Perforation wash tool and concept of open cavity being formed behind perforated casing (source B\&W, Inc., Composite Catalog 1980-81) . 
The most vulnerable part of the liner wash tool is the elastomer cup material. If a washer is moved while pumping fluid, the cups are held against the liner inside diameter with considerable force. Short life would be expected due to excessive wear on the soft rubber. With short packer spacing, to move perhaps 1 to $3 \mathrm{ft}$, stop, wash, then move again, would be time-consuming for liners with 1,000 ft or more of slots.

operator techniques in geothermal wells have not been evaluated, and operators have not been interviewed to determine their feelings about washing tool limitations or methods. Actually, liner washing is limited at present because long slotted liners are being installed in mud-arilled holes with nonfracture-type permeability in only a few areas. Those areas would be in East Mesa and Heber areas of the Imperial Valley below depths where cementing and perforating are not practical. In the Salton Sea and Brawley areas, hot wells flow strongly and sandstones are harder and are likely to be more fractured. If such wells can be drilled without plugging the fractures, they can probably flow strongly and give adequate rates without concern for matrix permeability cleanup. Baca Ranch completions are in this category. There, fractured "welded" volcanic tuff is drilled with a lightweight aerated fluid to prevent overbalance and fracture plugging. Slotted liners do not require washing to remove drilling muds.

A detailed study of liner washing should include perforation washing, as more geothermal wells will soon be developed in matrix permeability-type wells. Improved perforation washing or at least a better understanding of the phenomena involved would be of great interest to operators with wells requiring sand control. The thermal oil recovery interests, including those of Sandia Laboratories, would benefit greatly.

\subsubsection{Recommendations}

Further study of washing methods should evaluate the configuration and reliability of available tools, primarily the function of the elastomer packer cups and their longevity in typical geothermal applications. However, the emphasis should be to develop an understanding of the mechanisms directly affecting the critical near-wellbore portion of the exposed formation, that is, what are the shapes, velocities and hydraulic effects of jets resulting from various washer tool-liner slot (or perforation) combinations, at various casing-open hole standoff distances. Also, how these jets remove desired material and what the result of this action is on rock faces of various permeabilities and sand strengths must be determined. 
Such studies would be of great interest to the industry if they could be applied to perforation washing, as would be required for two objectives: to remove perforation cavity and tunnel plugging caused by gun debris and sand crushing; or, in unconsoliciated sands, to accomplish the above plus enlarge the cavities and create a volume behind pipe for gravel packing.

Specific tasks could include the following:

a. Construct a physical model to simulate, observe and measure effects of various washing methods. A simple model would consist of one slot or perforation impinging on a target sample which could be removed and tested for before and after permeability properties. A larger model could be designed to be packed with sand under various stress loads to simulate perforation washing.

b. Mathematically evaluate hydraulic effects and surface pressures created by various rates, configurations and fluid types.

c. Study slot configuration from the standpoint of mud and plugging removal, where sand control is not the objective. Since 0.25- or 0.1875-in.-wide slots have no sand screening capability, perhaps they could be made differently. The efficiency of drilled holes, straightsided and undercut slots, and possibly other designs, would be defined and compared.

d. Define the special needs for a geothermal well wash tool, particularly the need for more reliable elastomer packers. Evaluate field operations to determine if a tool that can be moved while pumping at high rates is available or, if not, if such a tool should be developed.

e. Determine application areas for improved liner or perforation washing. At present, wells of the type being developed in East Mesa and shallower Heber formations could benefit from improved cleanout and startup methods, whether slotted liners or cemented and perforated completions are used. 


\subsection{Geothermal Well Perforator Limitations}

\subsubsection{Implied Problem}

Lack of standard procedures for evaluation of well perforators at elevated temperature is the implied problem which will be discussed in the following subsections.

Performance of oil well explosives at elevated temperatures is vague at best. Temperature ratings of perforating guns are based on time/temperature limitations of basic explosive materials without regard to confining pressure, geometry or impurities. There have been few documented tests of perforating guns at elevated temperatures and the API has not established rating procedures. The lack of standard procedures for evaluation of well perforators at elevated temperatures has resulted in ambiguous temperature ratings by service companies who often claim an apparent technical advantage by rating perforating devices above their practical limits. Operators have no standard criteria for selection of well perforators for high-temperature applications.

\subsubsection{Discussion}

Virtually all of today's perforating devices are tested under standard procedures set forth in API-RP 43, now in its third edition (39). Section I tests determine physical characteristics of perforators under multiple-shot atmospheric shooting conditions, through casing or tubing, into precisely formulated and cured sand/cement targets. Penetration into the sand/cement target and casing hole diameter is recorded. Casing hole diameter is the most essential data recorded in this test, as it closely represents downhole conditions. Penetration into the sand/cement is less useful, as it may be affected by variations in the physical properties of the specific target.

Section II tests determine the physical characteristics and flow efficiency of perforations into a specific Berea sandstone target at pressure $\left(1000 \mathrm{lb} / \mathrm{in} .2\right.$ in core target and $1500 \mathrm{lb} / \mathrm{in} .{ }^{2}$ in the well), and temperature $\left(180^{\circ} \mathrm{F}\right)$. A number of parameters, including "Total Target Penetration" (TTP), "Total Core Penetration" (TCP), "Entrance Hole Diameter" and "Perforated Effective Permeability" are measured and recorded. This data is then combined with original Berea core data to compute:

a. "Core-Flow Efficiency" (CFE) - Ratio of experimental permeability ratio to theoretical permeability ratio for the same total core penetration and

b. "Effective Core Penetration" (ECP) - The product of total core penetration and core-flow efficiency. 
Penetration into the Section II Berea core target and CFE are widely accepted as a criterion for predicting performance of perforators in formations with various compressive strengths (40). However, entrance hole diameter, as measured in the Berea target, is less indicative of perforation performance in a well because a flat, mild steel plate is used in lieu of casing.

While RP 43 does set some valid criteria by which comparisons may be made, performance of perforators at bottom hole conditions is not adequately defined. Recent studies by Saucier and Lands concluded that penetration depths in various rocks (Wasson dolomite, Austin limestone, and Berea sandstone) are a complex function of the stress state of the rock (overburden and pore pressure) (41). This finding is contrary to the generally accepted belief that penetration is a function of formation compressive strength (40). The findings reported, and recommendations for improved API test procedures are currently being studied by the API. However, these studies are primarily aimed at establishing a relationship between formation properties and perforation performance. While such evaluation is needed, surface simulation of high pore pressures, formation overburden stress, and temperature will likely require elaborate (expensive) test equipment and take years to implement. Until adequate test facilities and procedures are available, an interim, less elaborate procedure is needed as a criteria for establishing temperature limitations of specific perforating devices.

Data from these tests (41) should be readily comparable to existing API RP 43 Section I and/or Section II data, and test procedures should be suitable (inexpensive and simple) for spot inspection of each manufacturing lot and after extended storage.

These tests should not be more severe than actual operating conditions for a given temperature, as this would lead to unnecessary use of expensive and generally less effective hightemperature explosives. The design of such tests must be based on an understanding of modern well perforating techniques and the manner in which these perforators are exposed to wellbore temperatures. These factors and proposed test procedures are discussed in subsequent subsections as follows:

a. Explosives and their nominal temperature ratings are compared to field test results.

b. Components of modern well perforators (blasting cap, primer cord, and shaped charges) are described.

c. Well perforating systems are categorized and exposure of each category to wellbore temperature is discussed.

d. Procedures for evaluation of well perforators at elevated temperature are proposed. 


\subsubsection{Explosives Used in Modern Well Perforators}

All perforating companies use the same type of explosives and obtain these explosives from the same suppliers. The most common explosives include: RDX, HMX, HNS and Picryl-sulfone $(\underline{42}, \underline{43})$.

RDX or Cyclonite (Cyclo trimethylene trinitramine) is the most widely-used explosive. It is currently available only from the United States government (Figure 2-21a). Users of RDX (and other explosives) mix various substances with the explosive according to its intended use. The perforating industry frequently uses steric acid (high-temperature wax used in dripless candles) and graphite. The steric acid acts as a binder and the graphite as a lubricant as the explosive is pressed into shaped charges.

HMX (Cyclo tetra methylene tetra nitramine), is frequently used in applications where expected temperatures exceed the practical limitations of $\operatorname{RDX}\left(340^{\circ} \mathrm{F}\right)$. Pure $\mathrm{HMX}$ (Figure 2-21b) is rated at $4000^{\circ}$ for $1 \mathrm{hr}$ and $350^{\circ} \mathrm{F}$ for $24 \mathrm{hr}$. However, the temperature capabilities of HMX are sensitive to impurities and may vary from one manufacturing lot to the next.

HNS (Hexa nitro stilbene) is used in high-temperature applications (Figure 2-21c). It is rated by the manufacturers at $500^{\circ} \mathrm{F}$ (several hours) and up to $550^{\circ} \mathrm{F}$ for shorter periods of time. HNS is used only in wells where temperature exceeds the practical limitations of other explosives as HNS is expensive and performance is 10 to 30 percent less than HMX or RDX.

Picryl-Sulfone (Hexanitrodiphenyl sulfone) is often used in lieu of HMX or HNS. It is relatively inexpensive, available in suitable quantities, and rated at $470^{\circ} \mathrm{F}$ for $1 \mathrm{hr}$. Performance of Picryl-sulfone is generally believed to be less than RDX or HMX but superior to HNS (Figure 2-21d).

Tacot(TM is a Dupont trade name. It is not currently available in standard perforating guns, but its $6500^{\circ} \mathrm{F}$ rating may eventually prove useful in geothermal and other high-temperature applications.

Temperature ratings for specific explosives as stated above and in Figure 2-21 are for pure explosives and are defined as that temperature at which an explosive either fails to initiate or self-initiates. This definition does not account for confining pressure, previous exposure at elevated temperature, impurities or geometry. In addition, there is no provision for determining the magnitude of reduced or improved performance as an explosive approaches its rated temperature. The lack of a standard procedure for defining the maximum practical temperature 
$\mathrm{C}_{3} \mathrm{H}_{6} \mathrm{~N}_{3} \mathrm{O}_{6}$

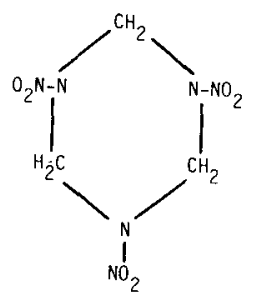

$340^{\circ} \mathrm{F}$ FOR $1 \mathrm{hr}$

$325^{\circ} \mathrm{F}$ for $24 \mathrm{hr}$

a) RDX WAS FIRST PREPARED BY HENNING IN 1899

(GERMAN PATENT 104.280) AND LATER BY VON HERTZ

IN THE UNITED STATES WHO RECOGNIZED ITS VALUE

AS AN EXPLOSIVE. IT WAS NOT USED IN EXPLOSIVE AMMUNITION ON A LARGE SCALE UNTIL WORLD WAR II.

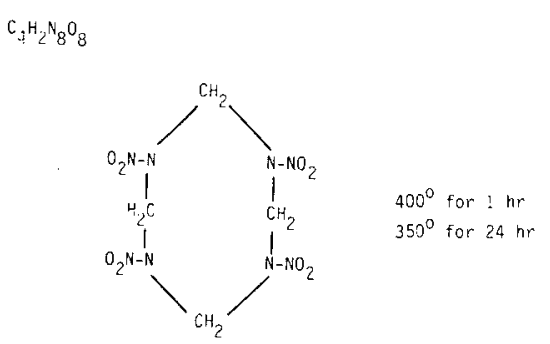

b) HMX WAS FIRST SEEN AS AN UNDESIRABLE IMPURITY CREATED DURING THE MANUFACTURE OF RDX. ALSO CALLED "HIS MAJESTY'S EXPLOSIVE," HMX WAS USED EXTENSIVELY BY THE FRENCH UNDERGROUND FROM 1941 TO 1944.

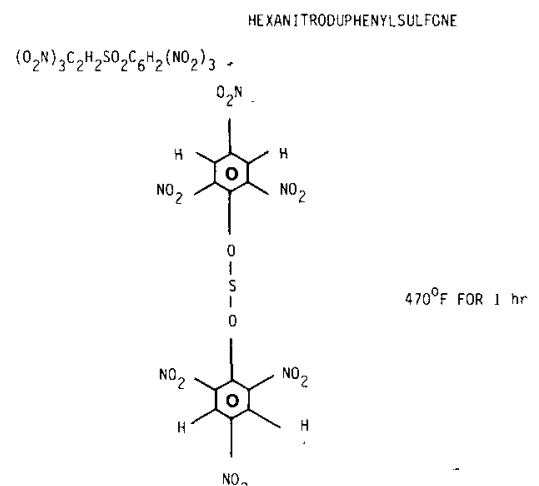

d) PICRYLSULFONE WAS FIRST PATENTED IN 1914 BY SPENGSTOFF IN GERMANY. IT WAS MIXED WITH T.N.T. AND USED FOR SHELLS, BOMBS AND TORPEDOES. AND THE F-111. THE COST OF HNS IS CONSIDERABLY HIGHER THAN MOST OTHER EXPLOSIVES DUE TO SLIGHTLY HIGHER CHEMICAL COSTS AND LOW DEMAND FOR OTHER APPLICATIONS. 
at which well perforators will perform as expected has led to ambiguous and conflicting claims by suppliers. Table 2-1 illustrates variations in published temperature ratings of well perforators with common explosive material.

TABLE 2-1. - Variations in temperature ratings by explosive type

\begin{tabular}{|l|c|}
\hline Explosive Type & Published Temperature Ratings \\
\hline RDX & $300^{\circ}$ to $350^{\circ} \mathrm{F}$ \\
HMX & $400^{\circ}$ to $425^{\circ} \mathrm{F}$ \\
HNS & $500^{\circ} \mathrm{F}$ \\
Picryl-Sulfone & $470^{\circ} \mathrm{F}$ \\
\hline
\end{tabular}

Published ratings of well perforators that contain RDX and HMX are less consistent than HNS or Picryl-sulfone.

CTC recently surveyed the six largest suppliers of perforating guns to ascertain procedures that are used to establish maximum temperature ratings of HMX and other explosives. The survey yielded very sparse documentation of temperature tests and many of these tests, as described below, indicated that published temperature ratings are too high.

Temperature test of HMX explosive performed by Jet Research Center, Inc. (JRC) in 1976 (44):

a. Test No. 1:

1. Heated 10 charges from ambient to $410^{\circ} \mathrm{F}-1 \mathrm{hr}$

2. Maintained $410^{\circ} \mathrm{F}$ for $1 \mathrm{hr}$

3. All charges performed as expected.

b. Test No. 2:

1. Heated 10 charges to $425^{\circ} \mathrm{F}$

2. Unspecified problems were encountered and test was aborted. 
Tests performed by E.I. DuPont in $1966(\underline{45})$ :

a. Test No. 1:

1. Heated $50 \mathrm{~g}$ of $\mathrm{HMX}$ from ambient to $350^{\circ} \mathrm{F}-\mathrm{l} \mathrm{hr}$

2. Sample was maintained at $350^{\circ} \mathrm{F}$ for $3-1 / 2 \mathrm{hr}$

3. Temperature was raised to $375^{\circ} \mathrm{F}$ over $1 / 2 \mathrm{hr}$

4. Sample was maintained at $375^{\circ} \mathrm{F}$ for $1-1 / 4 \mathrm{hr}$

5. Temperature was raised to $395^{\circ} \mathrm{F}$ over $20 \mathrm{~min}$

6. After $2 \mathrm{hr}$ and $15 \mathrm{~min}$, internal heating commenced which raised the temperature to $4350 \mathrm{~F}$, and within $10 \mathrm{~min}$, the sample detonated.

b. Test No. 2:

1. A $50 \mathrm{~g}$ sample of HMX was placed in an oven at $446^{\circ} \mathrm{F}$

2. When the sample temperature reached $410^{\circ} \mathrm{F}$, it fumed off.

c. Test No. 3:

1. A $50 \mathrm{~g}$ sample was placed in an oven at $425^{\circ} \mathrm{F}$

2. After 50 min, the sample fumed off.

d. Test No. 4 :

1. A $50 \mathrm{~g}$ sample was placed in an oven at $380^{\circ} \mathrm{F}$

2. After $16 \mathrm{hr}$, the sample had not fumed off, but it had lost 11.7 percent of its weight.

Following the survey, perforating companies in the Gulf coast area were requested to perform surface tests to confirm performance of perforating guns in a deep $18,000 \mathrm{lb} / \mathrm{in} .2,4050 \mathrm{~F}$ well that had been previously perforated without apparent success. Unfortunately, it was determined that only one company had surface facilities that could duplicate these downhole conditions and such tests would require significant expense and time. Therefore, an alternative test was used.

The test consisted of heating each gun from ambient to $405^{\circ} \mathrm{F}$ over a 1-1/2-hr time period (representing descent into well), and maintaining this temperature for an additional $1 / 2 \mathrm{hr}$ (gun 
positioning). The guns were then allowed to cool to ambient temperature and shot in identical surface targets. Three types of explosives were used in this test: HMX, HNS, and Picryl-sulfone. RDX was not tested because its temperature rating is well below 4050F. Guns containing Picryl-sulfone and HNS were tested without incident. However, charge performance was not consistent, that is, some charges had much shallower penetration than others. One gun containing HMX performed as expected, but a second gun failed as described below:

a. During the heating phase, a temperature anomaly occurred at $340^{\circ} \mathrm{F}$. Temperature rapidly and uncontrollably increased to $425^{\circ} \mathrm{F}$. This anomaly was the result of:

1. A faulty heating oil, or

2. An exothermal reaction in the HMX explosive, Figure 2-22.

b. An attempt was made to maintain a $405^{\circ} \mathrm{F}$ temperature for $1 / 2 \mathrm{hr}$. However, temperature anomalies continued until the heating coils were turned off.

c. When the gun was cooled to $326^{\circ} \mathrm{F}$, a low-order auto detonation occurred. Inspection of the gun indicated the following:

1. The steel gun housing was not perforated, but some swelling was evident.

2. Inspection of shaped charges and prima cord indicated that the detonation was low-order but one charge had reacted more violently than the others.

Unfortunately, verification of heater operation and additional tests were aborted due to heater damage. But, in any event, it was concluded that under some conditions, $425^{\circ} \mathrm{F}$ is beyond reasonable operating limits of $\mathrm{HMX}$, and a number of questions are apparently unanswered, such as:

a. What is a realistic temperature limit for use of HMX explosive?

b. Are other explosives as unreliable at their rated temperatures?

c. What effect do impurities have on explosive performance?

d. What effect does confining pressure have on performance? 


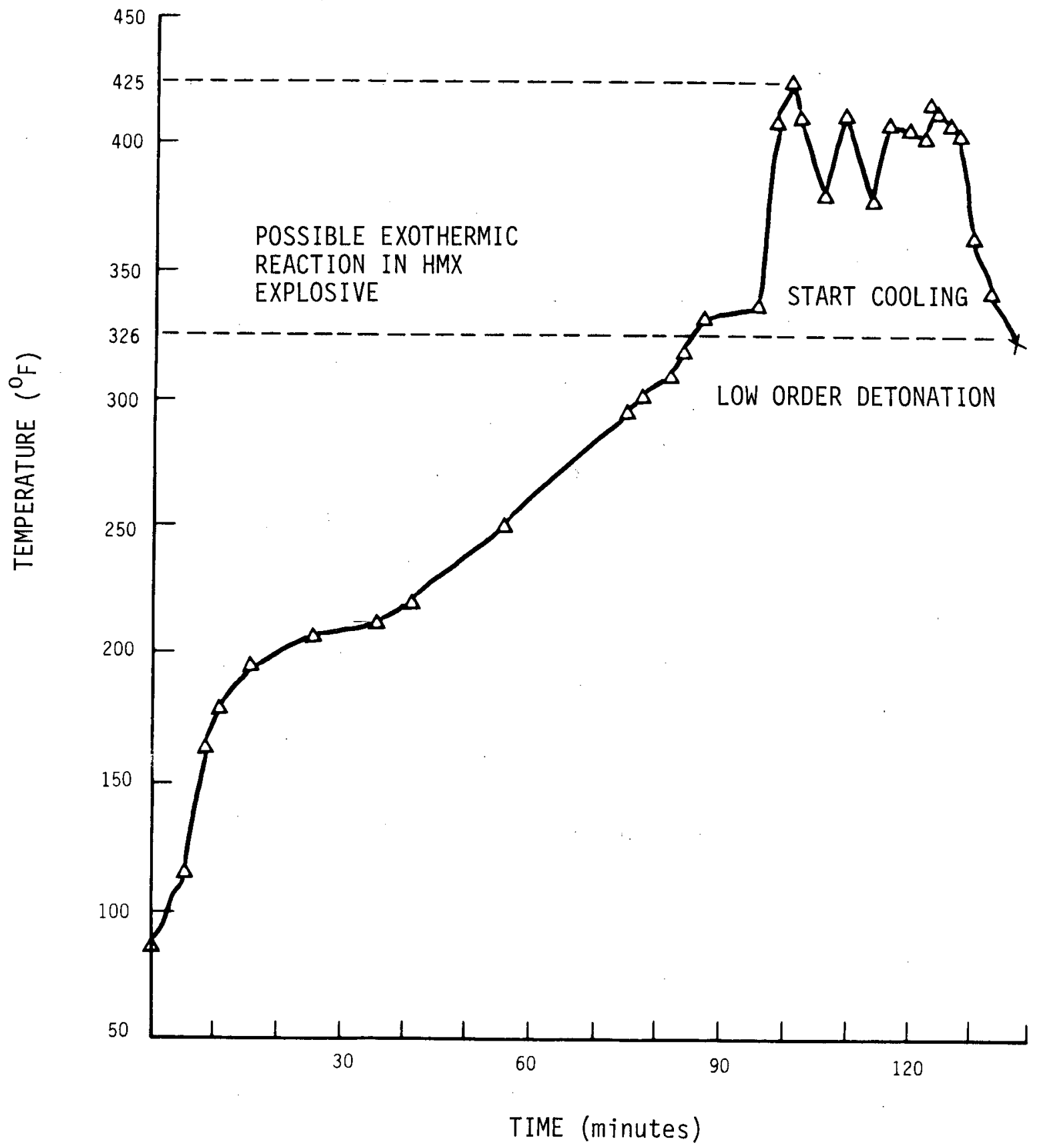

FIGURE 2-22. - Temperature response to a thermocouple attached to a 2-1/2-in. HMX perforating gun during temperature test.

$2-58$ 
e. What procedure could be used to establish temperature limitations of explosives?

f. What effect does geometry have on explosives, that is, does prima cord have different limitations than shaped charges that are formed under varying manufacturing procedures?

9. Do shaped charge liners function as expected at elevated temperatures?

\subsubsection{Components of Well Perforators}

Performance of well perforators at elevated temperatures may be affected by factors other than physical changes in explosive material. Most modern perforating systems consist of shaped charges, detonating cord and blasting caps. The blasting cap, or other primary explosive, initiates an explosive energy wave which travels along the detonating cord. This energy wave is transmitted to a precise geometric location in each shaped charge along its path. As the energy wave front advances in the shaped charge from the apex to the base of the conical liner, the metal liner is squirted into a focused fluid-like jetstream. The velocity of this jetstream as it moves through the target may exceed $5 \mathrm{mi} / \mathrm{sec}$ (46). Target penetration and hole diameter are a function of pressure and kinetic energy generated in the jet, Figure 2-23.

There are a number of theories describing the manner in which jets are formed in lined-cavity shaped charges. Evaluation of these theories is beyond the scope of this study. However, each theory emphasizes the importance of: geometry of charge design; velocity of explosive wave front; mass and characteristics of cone liner; and origin and direction of the explosive wave front relative to the axis of the cone liner.

In early shaped charges, carrots or slugs of liner material often plugged or restricted the perforation tunnel. These slugs were formed because the velocity of the fluidized metal in the jet is a function of its original location relative to the centerline of the liner wall. Material on the outer or explosive side of this liner moves less rapidly, forming a slug as material on the inner side of the liner enters the jetstream and moves forward into the target. In modern shaped charges, these slugs are eliminated or reduced through use of bi-metallic liners or powdered metallic cone materials.

The performance of shaped charges at elevated temperatures may be dramatically affected by any changes in geometry or the chain of events required to form long carrot-free jets. Uneven expansion of dissimilar materials and/or changes in physical 


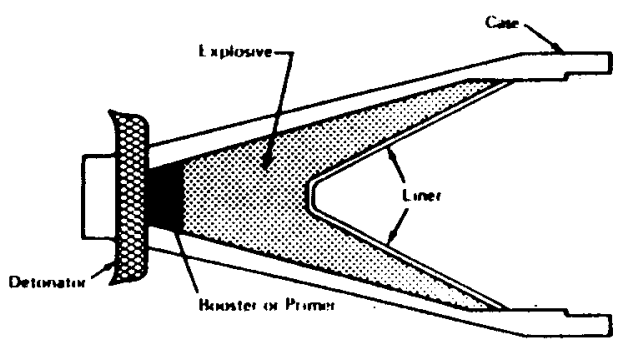

Mujor Components of

Shaped Charge Perforator

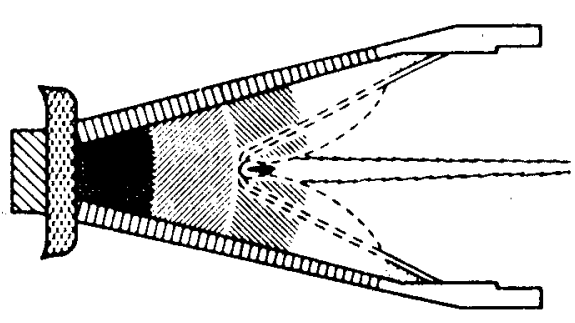

Wavefront collapses Liner.

The Liners inner surface disintegrates to form part of the Jet Stream.

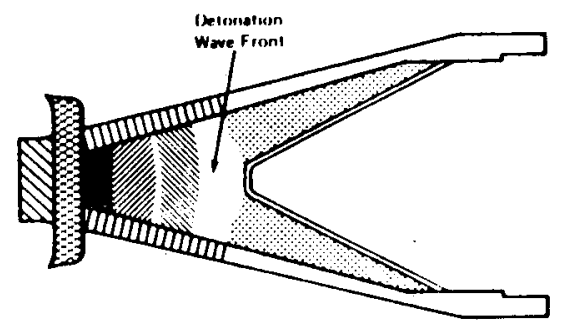

Detonation trivuls down

the charge and strikes

the Apex of the Cone

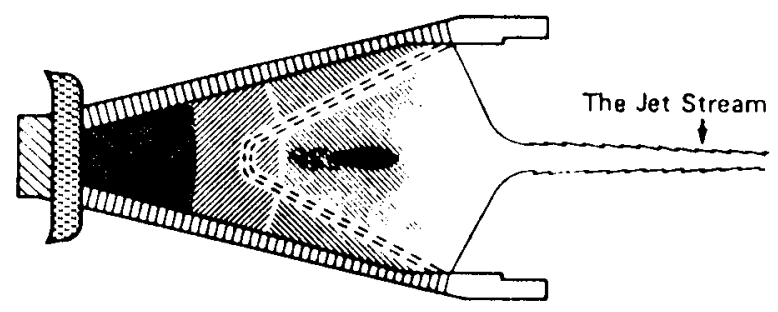

Advancing Wavefront forms

the Jet Stream. The outer sur

face of the Liner forms a Slug

or Carrot which follows the Je:

Stream

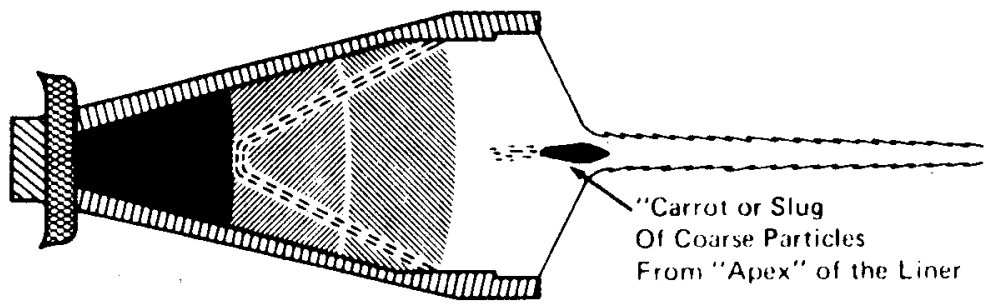

The fully developed Jet Stream penetrates a target, the Carrot follows with no contribution.

FIGURE 2-23. - Detonating stages of a typical shaped charge (courtesy of Dresser Atlas). 
properties of cone material at elevated temperatures may significantly alter charge performance. These alterations combined with physical changes in the explosive material may uniquely affect each specific charge design. Some charges may perform better at elevated temperatures, others may not. In effect, a valid evaluation of high-temperature perforating systems must include each component of the system, that is, blasting cap, prima cord, shaped charge and gun housing.

\subsubsection{Well Perforating Systems}

Performance of perforating systems in high-temperature wells may be affected by time, temperature and pressure. Surface evaluation should closely approximate conditions that will actually be encountered in a well with given bottomhole conditions.

For practical purposes, temperature within a wellbore increases linearly with depth. Time of exposure to these temperatures is a function of:

a. Time required to descend into well

b. Time required to position and detonate explosives.

In liquid-filled holes pressure increases with depth, but exposure of explosives to pressure and temperature is a function of gun design.

Modern perforating systems may be classified in three groups as listed below. The severity of exposure (time, temperature and pressure), for a given bottomhole environment is distinctly different for each of these systems.

a. Carrier and Expendable Carrier Guns - Explosives are enclosed in pressure-tight metal carriers run on wireline and detonated by electrical current, Figure 2-24. The metal housings protect explosives from well fluid (and pressure), maintain proper geometry between blasting cap, prima cord and shaped charges, confine explosive force for optimum penetration, prevent extraneous casing damage, and contain explosive debris. However, these carriers provide little heat shielding. Studies have shown that internal temperature increases to wellbore temperature in less than $5 \mathrm{~min}$ and the temperature differential as the gun is lowered at $20,000 \mathrm{ft} / \mathrm{hr}$ is only approximately $150 \mathrm{~F}$ (47). Internally-generated pressure from gas expansion and/or reactions (gas off) is contained within the carrier.

b. Expendable Guns - Shaped charges (enclosed in individual ceramic or other housings), prima cord (enclosed in a fluid-tight sheath), and blasting caps (enclosed in 
CARRIER GUN

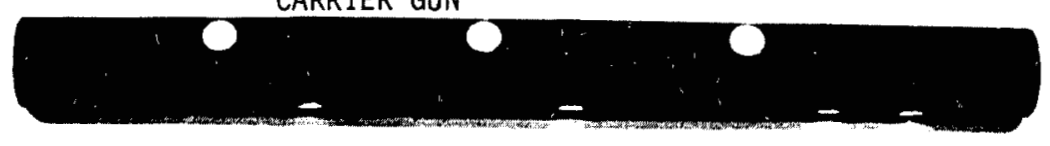

EXPENDABLE CARRIER GUN

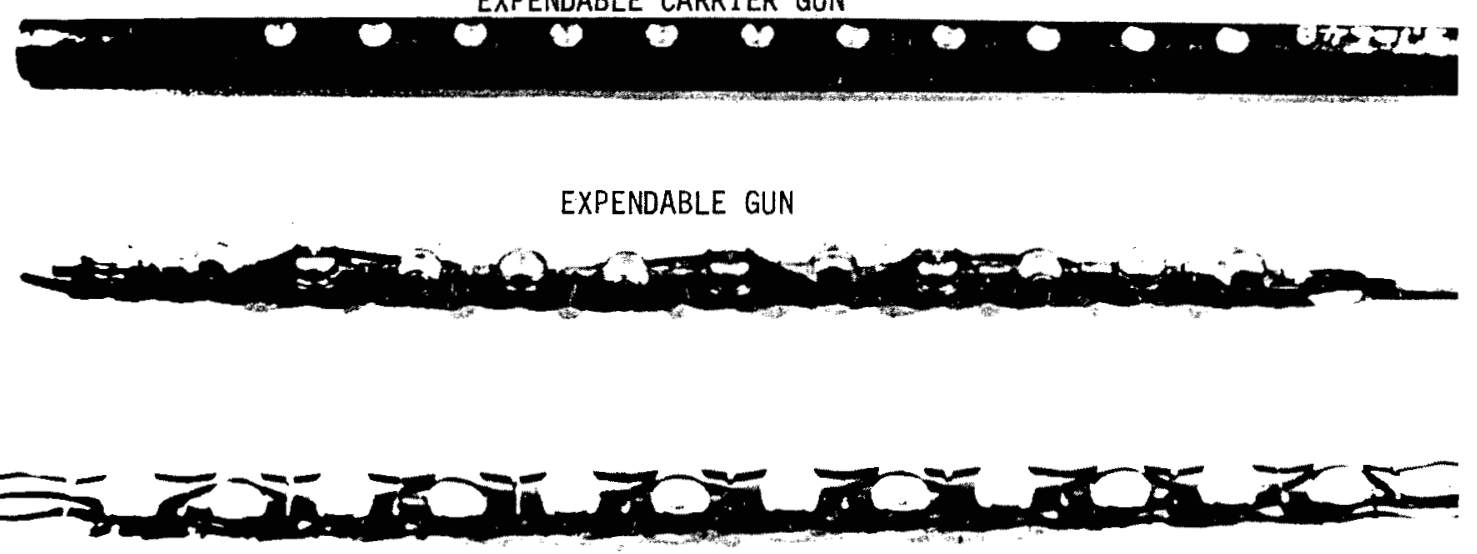

FIGURE 2-24. - Hollow steel carriers and expendable guns (courtesy of Welex).

fluid-tight housings), are exposed to well pressure and fluid as the gun is lowered into the well, Figure 2-27. A variety of strips or wires are used to attach the gun to the electrical wireline and maintain proper geometry between blasting cap prima cord and shaped charge.

c. Vann Guns - Shaped charges, prima cord and blasting cap are enclosed in fluid-tight metal carriers that are similar to wireline carriers. These guns are attached below tubing or drillpipe, lowered into the well, and detonated by dropping a weighted bar on the firing head.

Carrier, expendable carrier and expendable guns run on wireline may be lowered into a well at 10 to 20 thousand $\mathrm{ft} / \mathrm{hr}$. However, descent is often slowed by viscous completion fluids and wellbore restrictions. In deep $(20,000 \mathrm{ft})$ wells, descent may require up to $3 \mathrm{hr}$. During descent, the perforating system is exposed to uniformly increasing temperature that varies from ambient (surface) to bottomhole conditions. Once on bottom, the gun is continuously exposed to bottomhole temperatures until it is positioned and detonated. Unless problems are encountered, positioning and detonation require less than $30 \mathrm{~min}$. 
Vann guns run below drillpipe or tubing are lowered into a well at a much slower rate than wireline guns. Once on bottom, several hours may elapse prior to detonation. If these guns are run below production tubing, and surface control equipment must be installed prior to detonation, guns may face prolonged exposure to bottomhole temperatures. These exposure times are less consistent than with wireline guns, but most jobs are performed with less than $8 \mathrm{hr}$ of descent and $4 \mathrm{hr}$ of exposure at bottomhole temperature prior to detonation. Perforating done with drillpipe in low-pressure geothermal wells would be less timeconsuming.

\subsubsection{Recommendations for Evaluation of Well Perforators at Elevated Temperatures}

Procedures for evaluation of well perforators at elevated temperatures (API R.P.43 section III) are needed by industry. These evaluation procedures must provide criteria for establishing the following:

a. Practical high-temperature limitation of specific well perforators

b. Performance of well perforators as bottomhole temperatures approach the practical high temperature limitation as defined in Item a.

As stated in previous subsections, these tests should be readily comparable to existing API R.P.43 section I and/or section II data and test procedures should be suitable (inexpensive and simple) for spot inspection of each manufacturing lot and after extended storage.

Equipment required (Figure 2-25) to perform API R.P.43 Section II tests may be readily adaptable to high-temperature evaluation of well perforators. Some modifications, such as increased heater and pressure capacity, may be needed to perform such tests, but these changes are believed to be minimal. Definition of these modifications is beyond the scope of this study.

These modified high-temperature tests should be performed and data recorded as defined by API R.P.43 section II. However, these tests will be performed under thermal conditions similar to those encountered by a perforator in a well with given bottomhole temperatures, as summarized below for wireline (not tubing conveyed) well perforators:

a. Descent into welz - Uniform temperature increase from $70^{\circ} \mathrm{F}$ to bottomhole temperature over $3 \mathrm{hr}$

b. Positioning - Constant exposure to bottomhole temperatures for $1 / 2$ hr prior to detonation. 


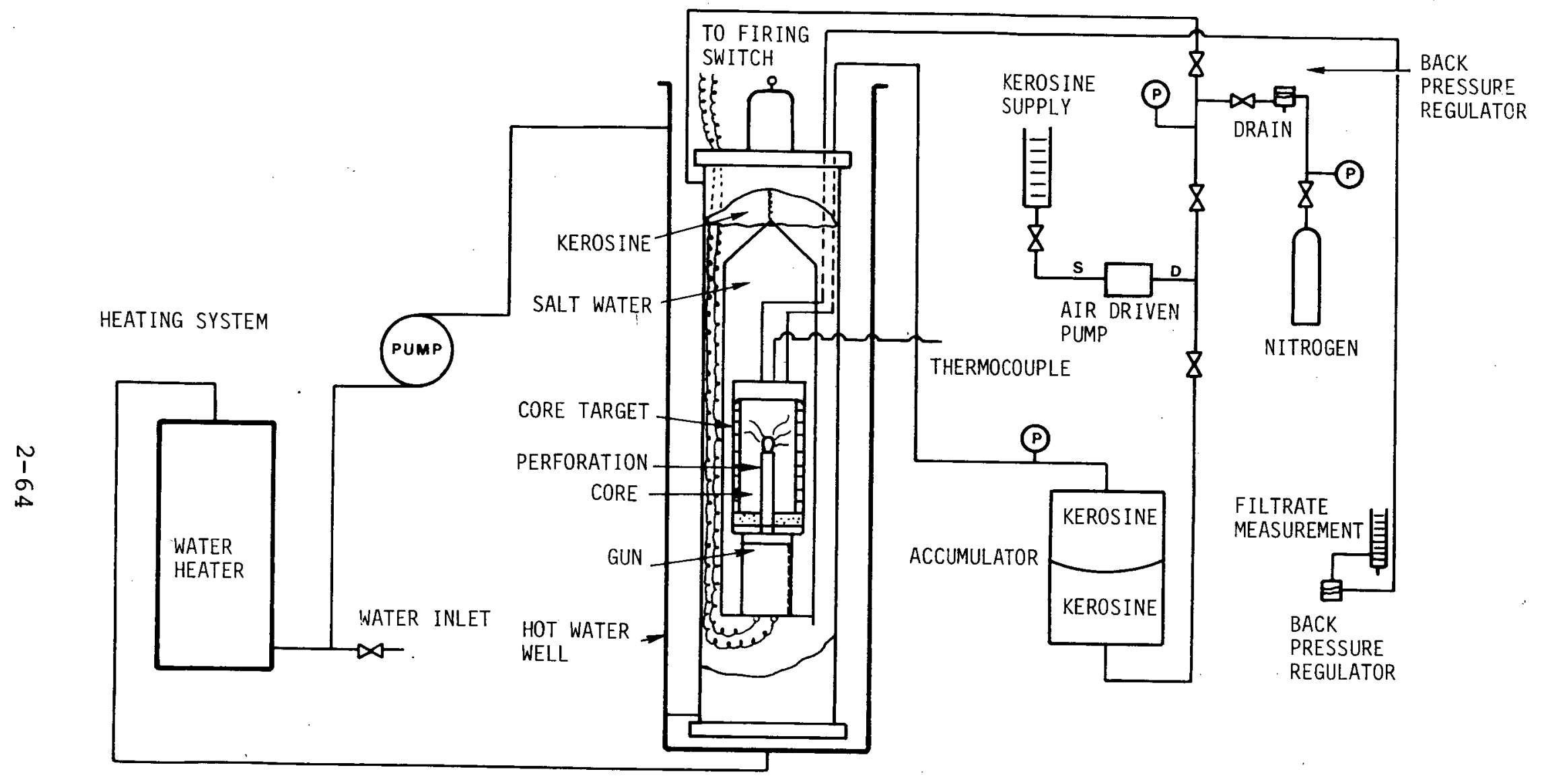

HOT WATER CIRCULATING SYSTEM

TEST CHAMBER

PRESSURE CONTROL SYSTEM

FIGURE 2-25. - Schematic diagram of gun perforator testing setup - Berea sandstone core target (courtesy of American Petroleum Institute). 
The definition of practical operating temperature for a given wireline perforator may then be defined as that bottomhole temperature at which the perforator fails to initiate, self-initiates, or performs with 25 percent or more reduction in penetration, hole size or core flow efficiency. 
• 


\section{TECHNICAL EVALUATIONS OF CASING CENTRALIZERS, CEMENTING PLUGS AND SLOTTER LINER WASHING}

\section{Summary}

The purpose of this section is to analyze in detail three technical problem areas which were identified in section 2 as areas of major concern in geothermal well completions. These areas are:
a. Centralizer Bow Frictional Heating and Wear
b. Cementing Plug Failure due to Frictional Heating and Wear
c. Slotted Liner washing.

While these are not the only areas where technical analysis and experimentation could be used to examine elements of the completions problems, this section shows methodology for examining select completion problems. Additional areas of concern are outrined and discussed in section 2.

\subsection{Centralizer Bow Frictional Heating and Wear}

\subsubsection{Introduction}

In completing a geothermal well, casings are cemented in place in order to strengthen the casing against buckling due to thermal elongation during startup and to protect it from corrosion due to hot thermal brines and gases. In addition, cementing prevents uncontrolled flow of thermal water and steam outside the casing $(\underline{48})$.

The task of cementing casings is made difficult by a number of factors. Such factors include: the elevated temperature and pressure of the geothermal well; the high viscosity of the cement; underpressure of the local environment such that cement and mud disperses into cracks and fissures in the surrounding environment; and the difficulty of centralizing the casing in the hole, especially when the hole is deviated (49). A wide variety of cementing equipment is used to assist in proper cementing of the casing (see Table 3-1). Improper cementing may occur if one or more of these mechanical aids fails to function (50).

Failure of the cement to surround and fully protect the casing can lead to buckling of the casing due to thermal loading*

*Figure 3-1 from Snyder shows casing buckling of an incompleted cementing job due to lost circulation. However, the problem in partial cementing is identical, that is, the casing will elongate and buckle into the unsupported media. 


\section{TABLE 3-1. - Digest of cementing equipment and mechanical aids}

\section{Comenting Equipment}

and Types

Floating Equipment

1. Guide Shoes

2. Float Collars

Automatic FIII-Up

Equipment

1. Float Shoes

1. Foal Shoes

Formation Packer

Tools

1. Formation Packer

Shoes

2. Formation Packer

Collars

Cementing Stage

Tools

2 Stage

3 Stage

Full Opening Tools

\section{- Plug Containers}

1. Quick Opening

2. Continuous

Cementing Heads

\section{Cementing Plugs}

1. Top and Bottom Wiper Plugs

2. Ball Plugs

3. Latch Down Plugs

\section{Casing Centrallzers}

Variable Typēs
Applicetion

Guides casing into well Minimizes derrick strain

Prevents cement flow back Create pressure differentials to improve bond

Catches cementing plugs
Same as Float Collars and Shoes except fill-up is controlled by hydrostatic pressure in annulus

Packer expands to protect

lower zones while cementing


more sections in separate stages

To hold cementing plugs in string until released.

Mechanical Spacer between Mud and cement (bottom plug) and cement and displacement fluid (top plug)

Center casing in hole or provide minimum stand-off to improve distribution of cement in annulus, prevent differential sticking

Remove Mud cake and circulatable mud from well bore.

Aid in creating turbulence Improve cement bond
When required to cement two or

\section{Placement}

First joint of casing

1 joint above shoe in wells less than 6,000 ft 2-3 joints above shoe in wells greater than $6,000 \mathrm{ft}$

Same as Float Collars or Guide Shoes

First joint of casing

As hole requirements dictate

Based on critical zones and formation fracture gradients

Top joint of casing at surface of well

Between well fluids and cement and 200 feet above and below pay zones; 1 per 3 joints in open hole to be cemented

Crooked hole-Variable with deviation

\section{Scratchers or Wall} Cleaners
1. Rotating

2. Reciprocating
Place through producing formations and 50 to 100 feet above. Rotate pipe 15 to 20 RPM Placement is same as rotating Reciprocate pipe pipe 10 to 15 feet off bottom 
(51) (Figure 3-1). Attempts to correct these problems have been expensive (52), leading to increased cost of casing repair (53), and in some cases to the abandonment of the well (54). At an average well cost greater than $\$ 1,000,000 \quad(53), 10 s 5$ of a well due to cementing problems is an expensive proposition.

The purpose of this report is to examine the role of the casing centralizer in the well cementing problem. While it is clear that casing centralizers are only a part of the cementing mechanical aids, their failure to centralize a casing during cementing can lead to failure of the well (55). Thus, we will examine possible failure modes of the casing centralizer.

\subsubsection{Casing Centralizers}

Typical casing centralizer bows are manufactured to API Standard 10D (February, 1973) (56) which specifies the maximum side force which a centralizer bow must exert as a function of the casing or hole diameter into which the bow is to be inserted. For a straight hole, the centralizer bow exerts minimum force (57)

(approximately $500 \mathrm{lb}$ ) to hold one casing centrally within another casing or hole. When the casing is forced off center, such as in a deviated hole, the restoring force exerted by the bow quickly rises (Figure 3-2). To minimize the resulting eccentricity of the pipe, it has been recommended that hole deviation not exceed $2.5 \mathrm{deg} / 100 \mathrm{ft}$, although in practice, deviations up to $10 \mathrm{deg} /$ $100 \mathrm{ft}$, have been measured (58).

At a deviation rate of $2.5 \mathrm{deg} / 100 \mathrm{ft}$, typical side loading on centralizer bows can reach 1600 to 2500 lb (56), depending upon several factors, including the amount of casing hanging below the deviated portion of the hole (Figure 3-3). Since the force is spread over several bows (typically three bows contact the high force side), a maximum force on individual bows will reach 800 to $12001 \mathrm{~b}$ force.

Centralizer bows are manufactured from hot-rolled annealed strips, AISI 50B46, or Type 4130 steel, and typical hardness of these spring steel bows is Rc 40 to 44 (59) (Knoop Hardness is 400 to $\left.440 \mathrm{~kg} / \mathrm{mm}^{2}(60)\right)$. Typical cross-sections of centralizer bows are $0.2 \mathrm{in}$. thick by $1.5 \mathrm{in.}$ wide and the bows come in various lengths up to $30 \mathrm{in.}$. long (see $B \& W$ Incorporated catalog pages enclosed, Figure 3-4).

Centralizers are price-competitive. The majority of centralizer bows are manufactured by the least expensive method. In general, their material properties have not been optimized for the geothermal environment, although they meet API Standard 1OD. 


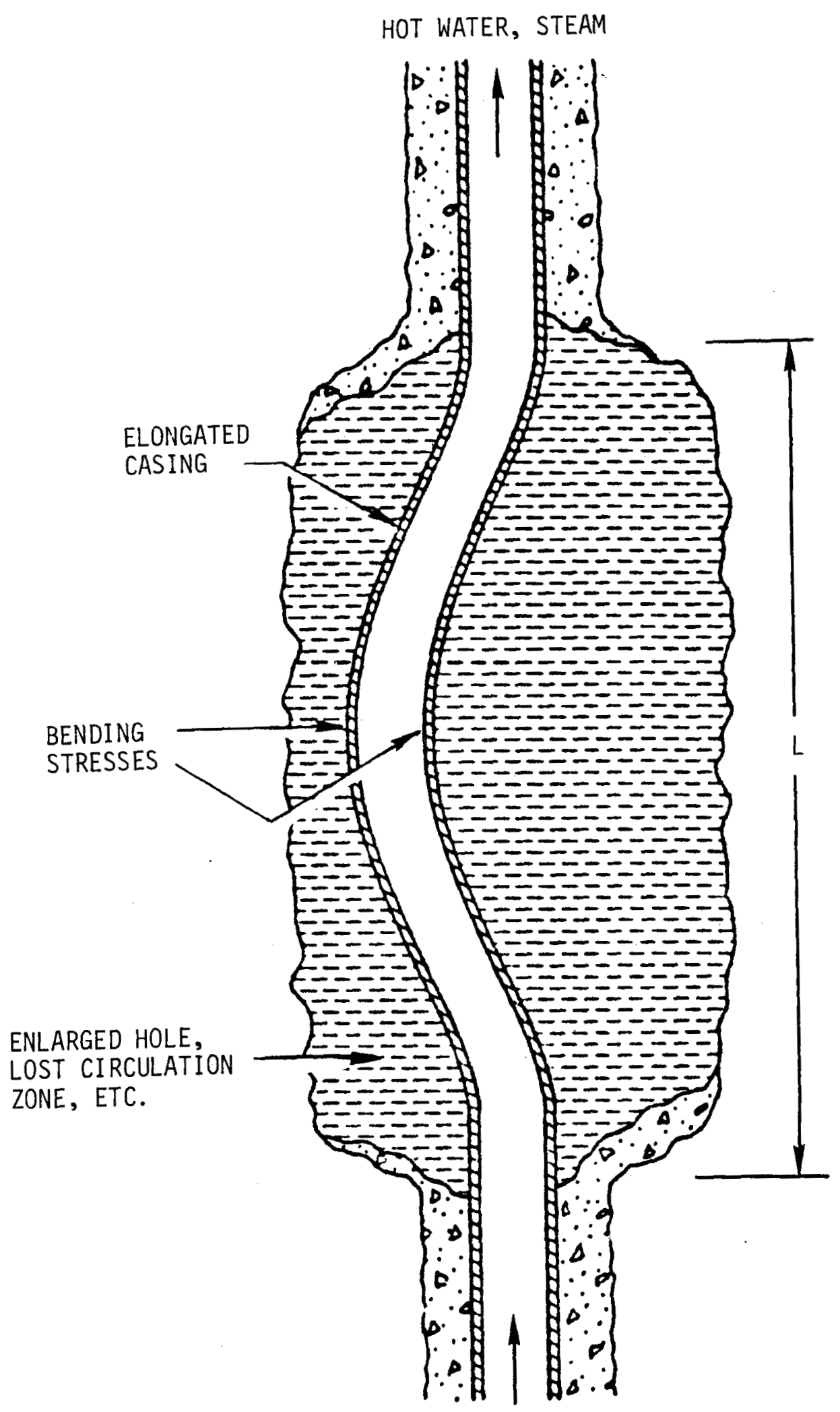

FIGURE 3-1. - Casing buckling of unsupported pipe with heating (after snyder) (49). 


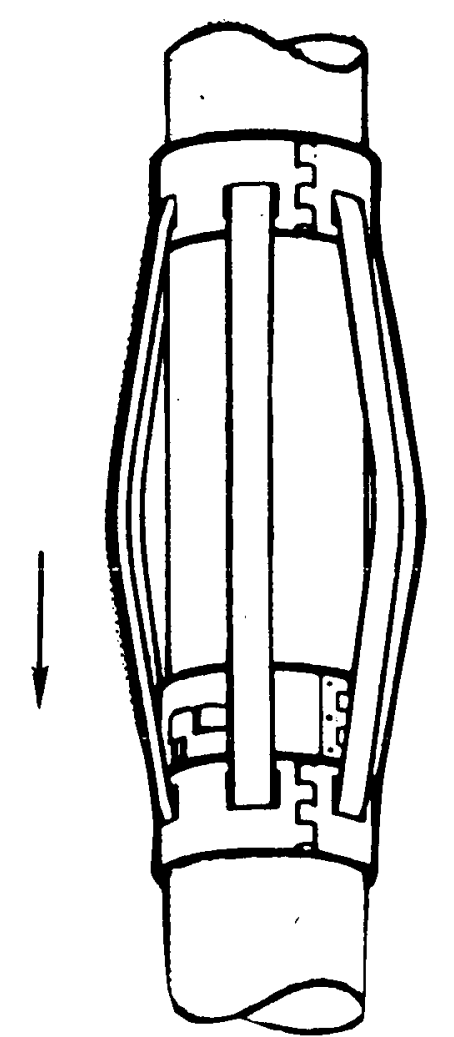

HINGED

CENTRALIZER

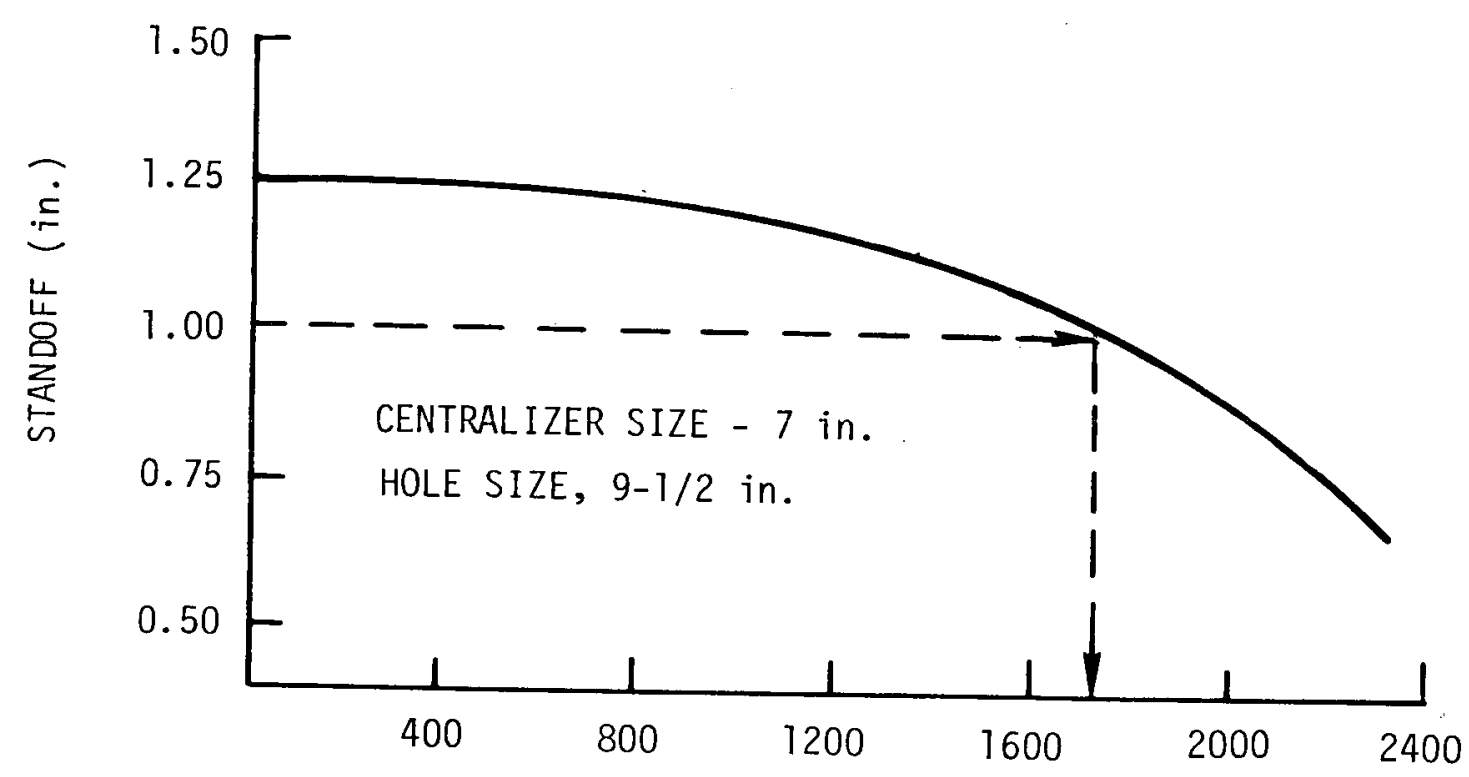

RESTORING FORCE (1b)

FIGURE 3-2. - Example of spring-bow centralizer contained by stop collar so that device is pulled into hole. Load-deflection curve for a centralizer gives lateral force on casing at various deflections. For the example curve shown, it takes over $1700 \mathrm{lb}$ to move the casing $1 / 4$ in. Off center (after Suman) (55). 


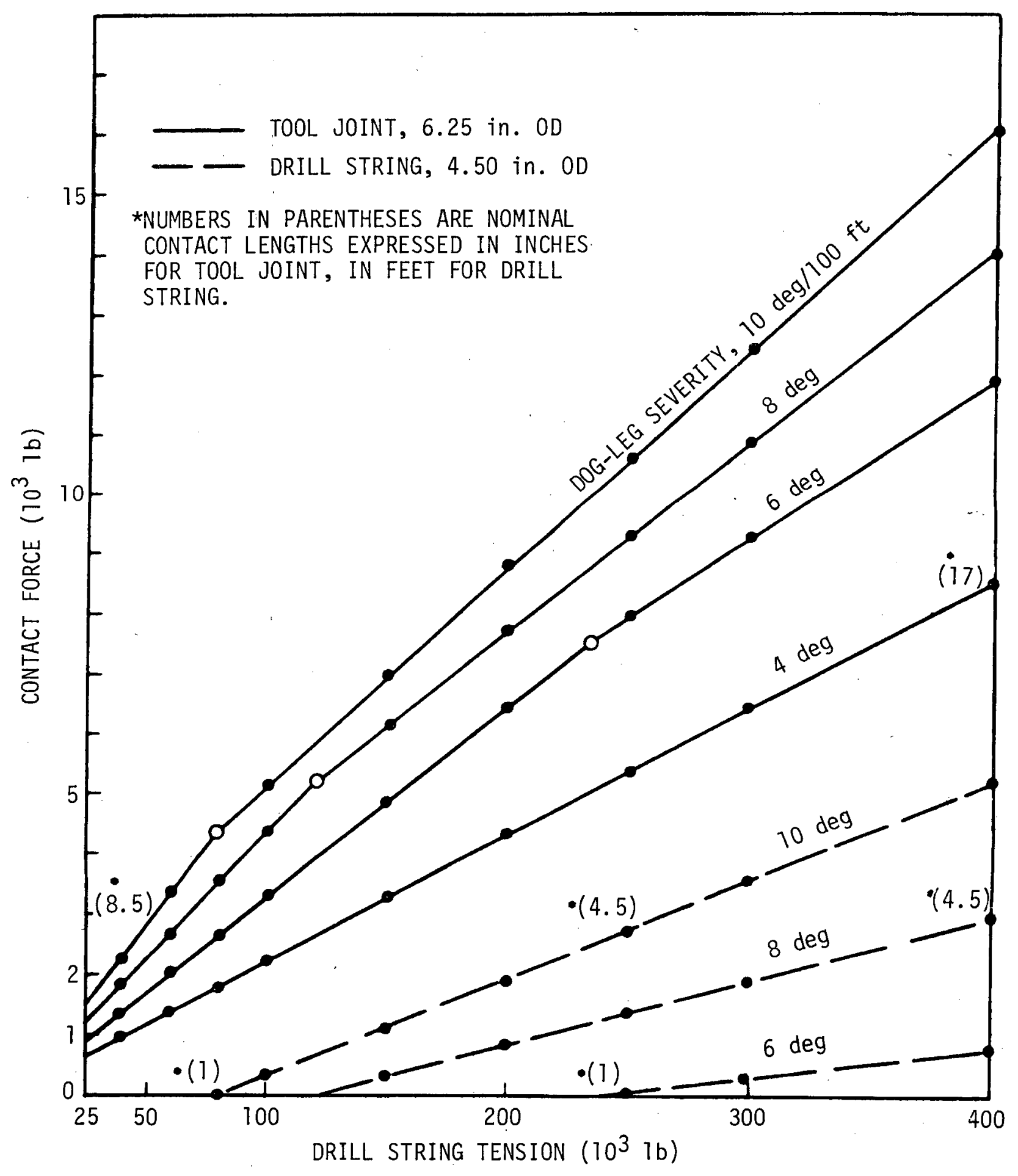

FIGURE 3-3. Tool-joint and drillstring contact forces in dog-legs versus axial tension 4.5 in. drillpipe (after Bradley, et al.) (다). 
LEGEND (Bow or Rod Cross.Sectional Dimensions)

- $=1 / 8$ " $\times 3 / 4 "$

$0=1 / 8 " \times 11 / 2 "$

$x=.170^{\prime \prime} \times 1 \frac{1 / 2}{}$

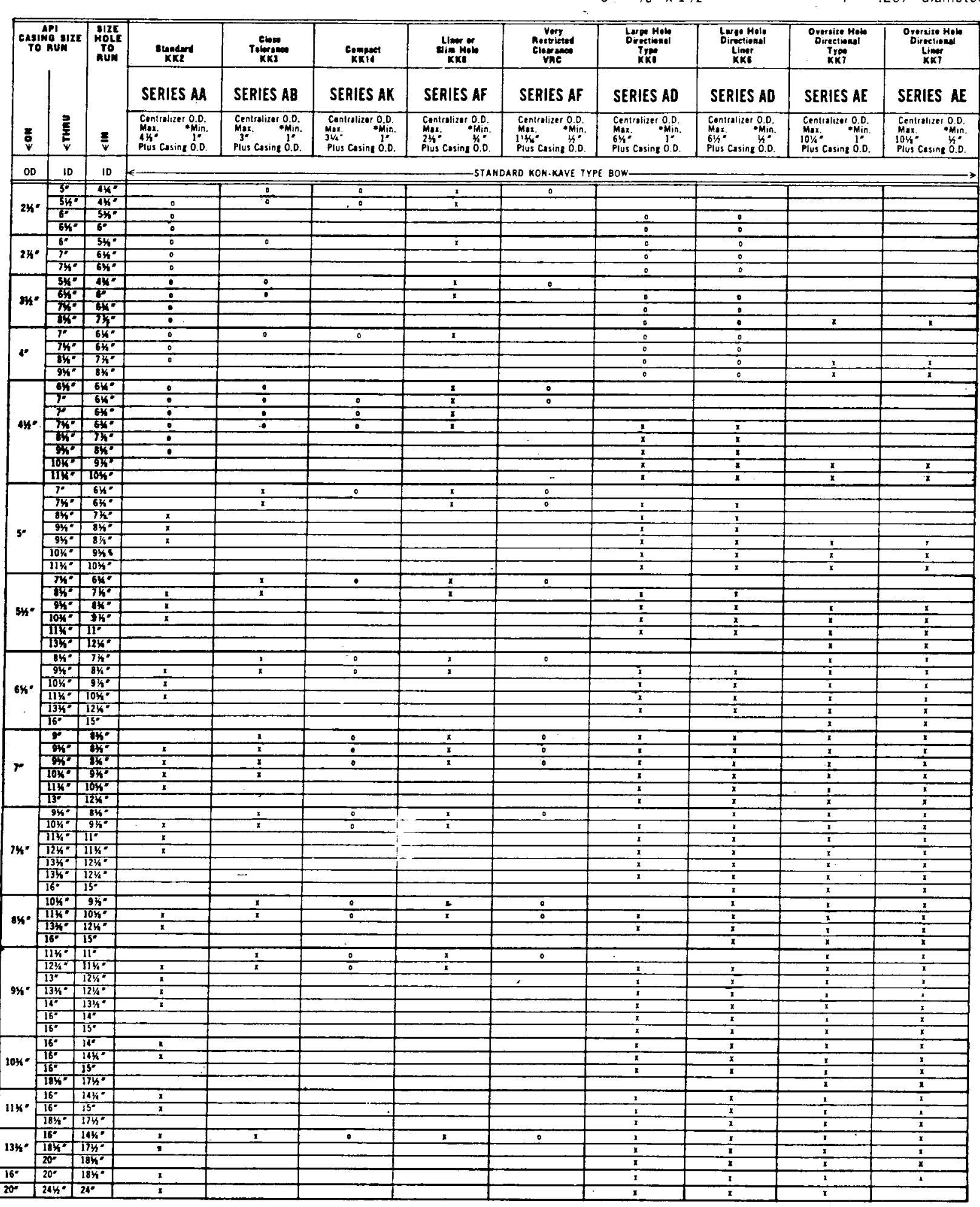

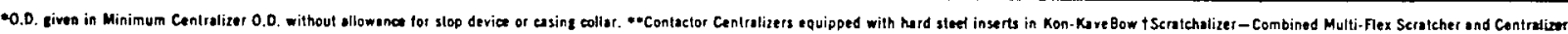

FIGURE 3-4. - B \& W basic centralizer selection guide. 
These recommendations, indicated by $\bullet, 0, x$, and $r$ (see Legend), are to be considered only as

general guides. Those for bow-type centralızers are arbitrarily based on the following criteria

(1) Maximum unrestricted nominal 0.0 . of centralizer at least $1 / 2$

In every case the appropriate force and spacing curves must be considered in relation to individual hole characteristics to ensure adequate minimum stand-off of casing from the wall of the hole.

As long as this stand.off is maintained. centralizers can function successfully in open-hole sizes larger than their own unrestricted maximum 0.0 stricted 0.0 . izer to run in with weight equivalent to that of $60^{\circ}$ of mean-weight casing.

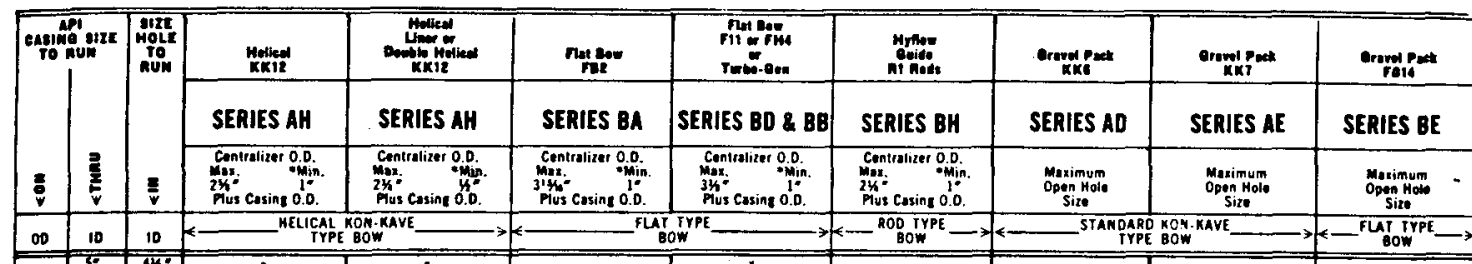

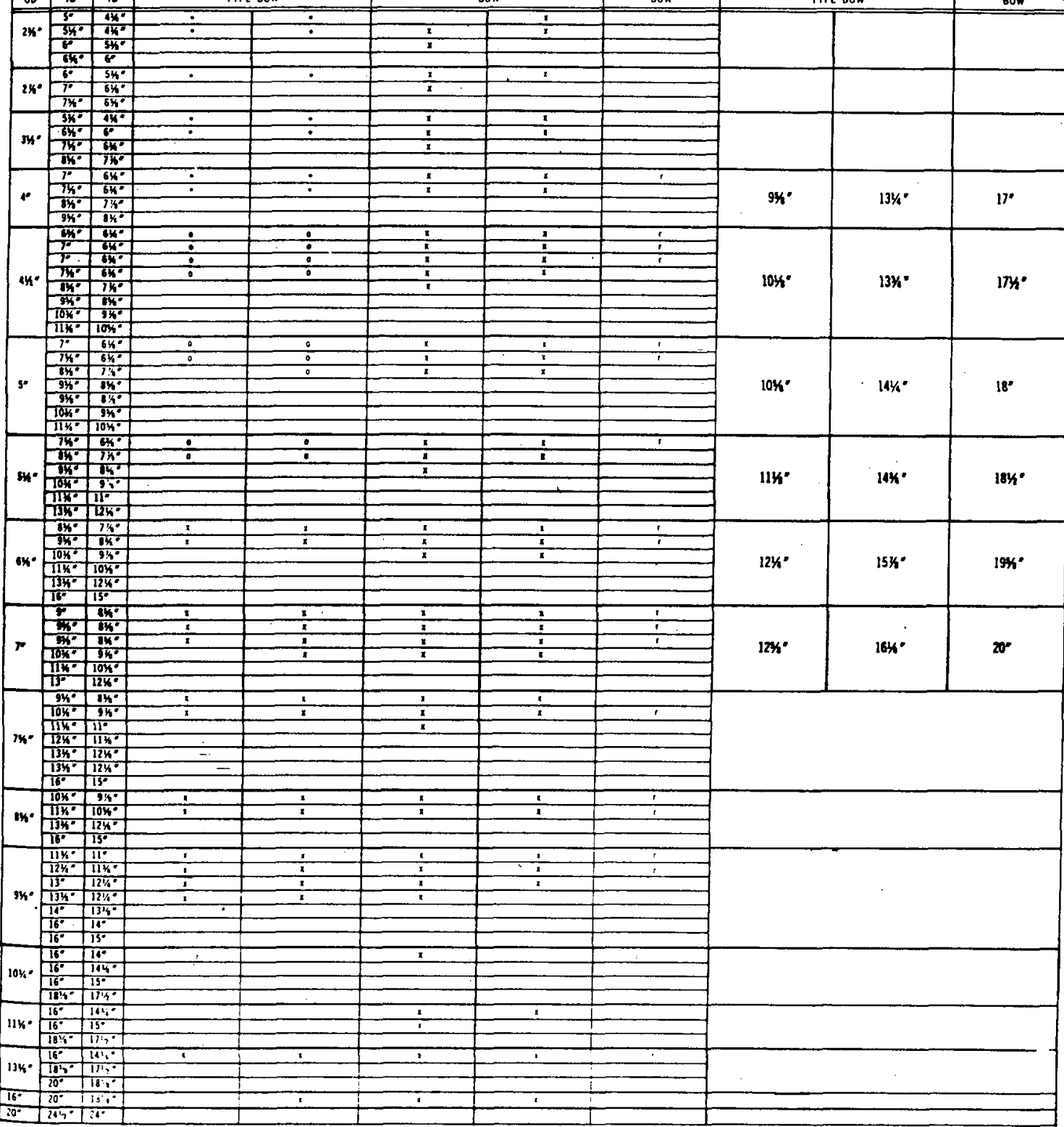

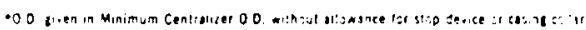

FIGURE 3-4. - B \& W basic centralizer selection guide (continued). 


\subsubsection{Geothermal Well Completions}

The geysers in northern California and most wells in the Imperial Valley in southern California produce dry steam at approximately $500^{\circ} \mathrm{F}\left(260^{\circ} \mathrm{C}\right)$. The Cerro Prieto Field (northern Mexico) produces hot water between $350^{\circ}$ to $650^{\circ} \mathrm{F}$ (177 to $243^{\circ} \mathrm{C}$ ) (49). Typical wells are 5000 to $9000 \mathrm{ft}$ deep, which is considered to be relatively shallow. These wells are under-pressured with respect to the hydrostatic head resulting from a column of water or mud. Low pressure and moderate depths lead to completions with the lowest strength casings.

Well Casing Plans

A typical casing plan for a geothermal well is shown in Figure 3-5 after Carson, et al. (53). The casing plan for a representative East Mesa Well Model (Imperial Valley) starts with a 20-in. conductor cemented within a 26-in. hole to a depth of $100 \mathrm{ft}$. Next, a 17-1/2-in. hole is arilled to $1700 \mathrm{ft}$ and a 13-3/8-in. casing is cemented to the surface. Centralizers are used to align the 13-3/8-in. casing within the 17-1/2-in. hole prior to cementing.

\begin{tabular}{|c|c|}
\hline HOLE SIZE (in.) & PIPE SPECIFICATIONS \\
\hline 26 & $\begin{array}{l}20 \text { in. } 941 \mathrm{~b} / \mathrm{ft}, \mathrm{H} 40 \quad 100 \mathrm{ft}] \\
\text { CONDUCTOR }\end{array}$ \\
\hline $17-1 / 2$ & $\begin{array}{l}13-3 / 8 \text { in., } 48 \text { lb/ft, H40 BUTT } 1700 \mathrm{ft} \text {.......p } 200 \mathrm{ft} \\
\text { SURFACE CASING }\end{array}$ \\
\hline $12-1 / 4$ & $\begin{array}{l}9-5 / 8 \text { in., } 36 \mathrm{lb} / \mathrm{ft}, \text { K55 BUTT } \\
\text { INTERMEDIATE CASING/LINER }\end{array}$ \\
\hline $8-3 / 4$ & $\begin{array}{l}7 \text { in. } 291 \mathrm{~b} / \mathrm{ft} \text { N80 LT\&D } \\
\text { SLOTTED LINER }\end{array}$ \\
\hline
\end{tabular}

FIGURE 3-5. - Casing plan for representative East Mesa Well (Imperial Valley) model. 
Next, a 12-1/4 in. hole is drilled to 5500 ft from the approximate termination of the cemented surface casing. A centralizer bow is mounted on the 9-5/8 in., $36 \mathrm{lb} / \mathrm{ft}$ casing at intervals depending upon whether the hole is straight or deviated (56). From the $B \& W$ Centralizer Selection Guide (Figure 3-4), one might select the standard KK2 Series AA centralizer bow to run through a $13-3 / 8$ in., $48 \mathrm{lb} /$ ft casing (12.715 in. ID) into a $12-1 / 4$ in. hole. Total travel through the $13-3 / 8$ in. casing would be $1700 \mathrm{ft}$. Total travel in the $12-1 / 4$ in. hole would be $3800 \mathrm{ft}$ (5500 minus $1700 \mathrm{ft}$ ).

Next, an 8-3/4 in. hole ( $8.75 \mathrm{in.}$. ID) would be drilled from the cemented end of the $9-5 / 8$ in. intermediate casing/liner to a depth of $7600 \mathrm{ft}$. A 7-in., $29 \mathrm{lb} / \mathrm{ft}$ N80 slotted liner would be slid into this hole. Again, a standard KK2 bow, Series AA might be chosen to slide through the 9-5/8 in. casing into the 8-3/4 in. hole. Maximum travel of the centralizers for the slotted liners would be $3800 \mathrm{ft}$ through the inside diameter of the 9-5/8 in. (8.921 in. ID) and $2100 \mathrm{ft}$ through the $8-3 / 4 \mathrm{in}$. hole (8.75 in. ID).

Use of Centralizer Bows in the Geothermal Well

The centralizer bows would travel, typically, through a loose fit casing, supplying minimal restoring force, and, therefore, minimal frictional heating and abrasion. The centralizer bows would then travel through a tighter-fit open hole where the bow would contact the side wall of the hole. Temperature rise of the bow against both the casing and the open hole would depend to the greatest extent on the following: the hole deviation; the composition of the hole wall (rock versus sand, clay, etc.); and the temperature of the wall, and the circulating mud. Typical wall temperatures would be $240^{\circ} \mathrm{F}$ due to the cooling effect of circulating fluids prior to cementing (6l). Wall temperatures up to $400^{\circ} \mathrm{F}$ have been reported (49).

The rate at which a centralizer bow is run downhole will depend to the greatest extent on how quickly a drill string or casing can be made up. From the Union Oil drilling record at Baca Ranch (62), we find that a typical trip in and out of Well No. 5 took $3 \mathrm{hr}$ for $3090 \mathrm{ft}$. For the total distance traveled of $6180 \mathrm{ft}$, this is an average velocity of $34 \mathrm{ft} / \mathrm{min}$. This translates to slightly less than one casing length (40 ft) per minute.

If we assume that this is close to average trip speed, we can set limits on the friction and wear problem on centralizers by assuming that the rate of travel downhole will be as slow as one casing per $2 \mathrm{~min}$ and as fast as two lengths per minute. This would give speeds of $20 \mathrm{ft} / \mathrm{min}$ and $80 \mathrm{ft} / \mathrm{min}$. 
Completion practices vary with location (48). Early salton Sea wells were cemented in stages to total depth. In northern Italy, geothermal steam wells were cemented through the production casing, but the wells were completed with an open hole. Imperial Valley current wells are cemented to the end of the production casing, but the wells were completed with an open hole. Present Imperial Valley wells are cemented to the end of the production completed with an open hole approximately 3000 ft deep (63) (see Figure 3-6). In Baja California, Cerro Prieto Field, slotted production liners are hung from the intermediate casing in an open hole. Completion fluids are used to control sand infiltration around slotted liners.

In the Imperial Valley, intermediate casings are typically type K55 (53). In northern Italy, the production casing is J-55 (48). In Baja California, the intermediate casing is type $\mathrm{N}-80$ (48). In northern California Geysers Area, the surface casing is K-55. These are representative casing materials over which a centralizer bow would be run. The J-55 casing has a yield strength of $55,000 \mathrm{lb} / \mathrm{in} .^{2}$. Although the hardness of casings is usually not rated, we can estimate from the casing data (64) and the Mechanical Engineers Handbook (65) that the Brinell Hardness number for casing steel is approximately 140 for Type

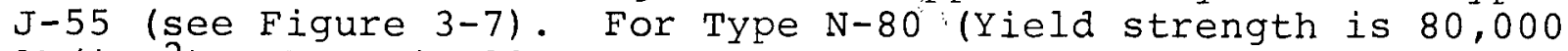
lb/in.2), the Brinell Hardness number would be approximately 170 . The corresponding Diamond Pyramid Hardness (Knoop) is approximately 150 (60) for J-55 and 180 for $N-80$. This hardness is a direct measure of the wear resistance of the casing steel.

Side Walls - the Centralizer Bow Environment

Side wall of the open hole varies with location (66). In the Geysers, completions are made in hard Graywacke. Roosevelt Hot Springs completions are made in fractured volcanic rock. At Baca Ranch and Deep Wells in Salton Sea and Cerro Prieto, formations are fractured sands or "welded" volcanic dusts which can become unstable and thus require cementing for completion. Another type of surface is found in the Hot Dry Rocks (HDR) program where granitic structures are found in the Jemez Mountains of northern New Mexico (61).

All of the geothermal open holes represent environments which are very hard and abrasive. One such material, fractured silica sands, has a hardness of approximately $800 \mathrm{~kg} / \mathrm{mm} 2$ whereas diamond has a hardness of $8000 \mathrm{~kg} / \mathrm{mm}^{2}(67)$. This is approximately 7 on the Mohs scale of Hardness where diamond is 10 . 


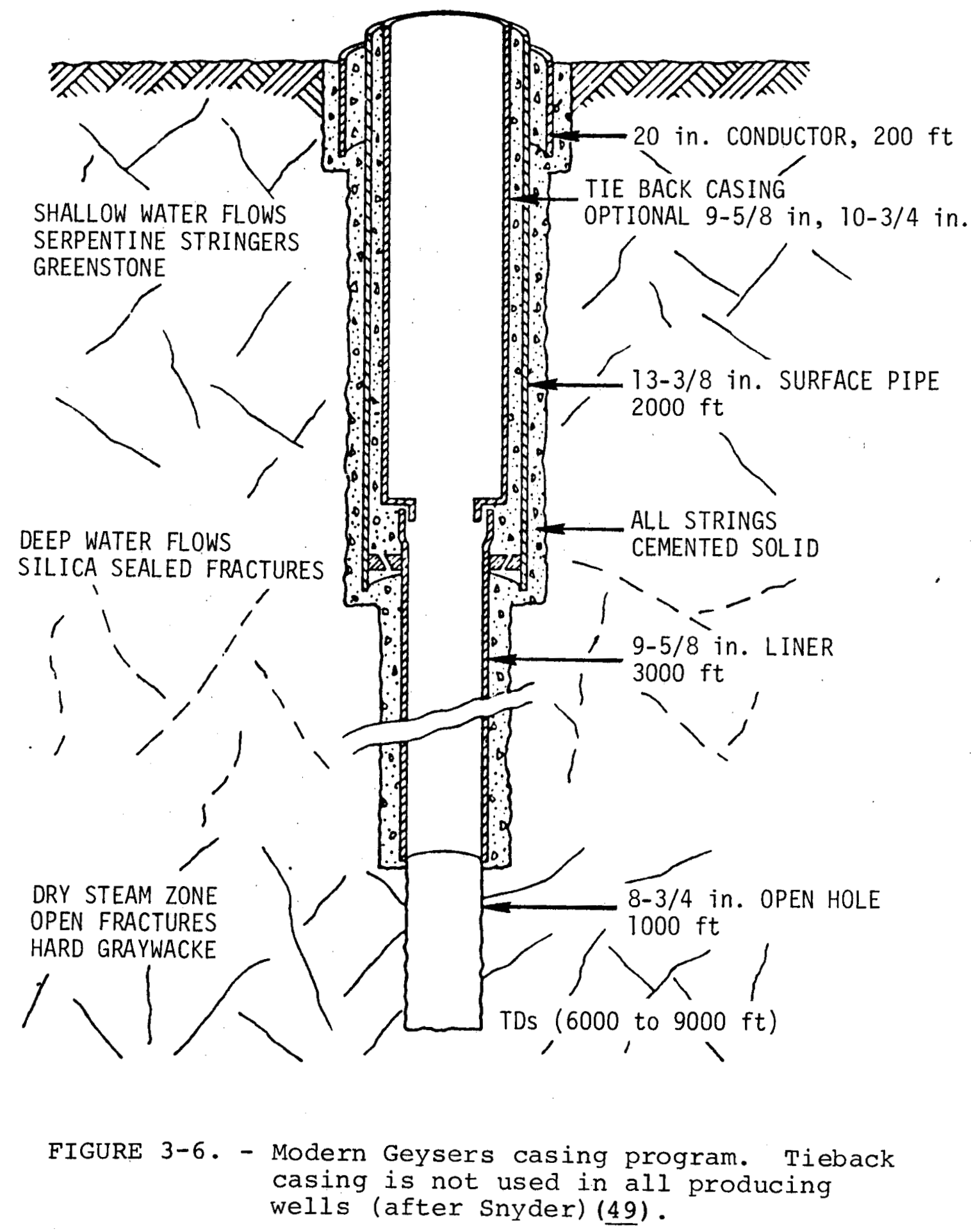




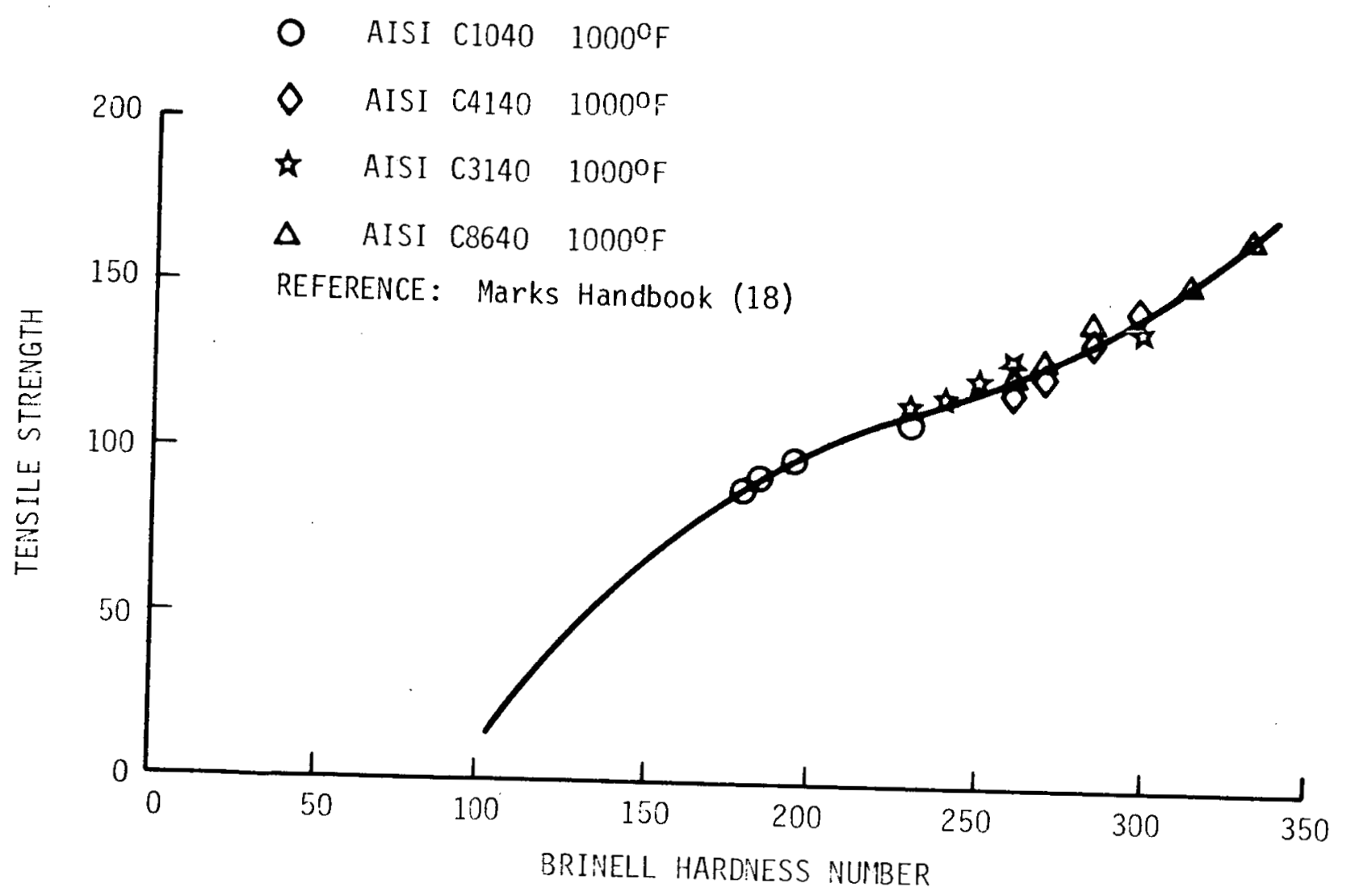

FIGURE 3-7. - Tensile strength versus Brinell hardness. 
Geothermal Well Deviations

Geothermal wells are typically drilled at a 40-acre spacing per well. Although the wells are drilled near normal at the surface, their direction drifts "wildly" (68) so that directional drilling is needed to correct deviation and/or direction in order to end up in the correct location. Deviation of $2 \mathrm{deg} / 100 \mathrm{ft}$ with a maximum buildup of $15 \mathrm{deg}$ is an acceptable limit (58). In some cases when a dry well has been drilled, the well $\overline{i s}$ plugged back to the bottom of the 9-5/8-in. Iiner where a new leg is directionally drilled in the opposite direction. In some cases, multiple bottoms have been put on a well in order to make it produce $(\underline{68)}$.

\section{The Case of Interest}

In examining the interaction between the centralizer bow and the geothermal well, there are three cases of potential interest, as follows:
a. Straight geothermal well
b. Deviated geothermal well - 2 deg/100 ft; 15 deg maximum
c. Highly deviated well - up to $10 \mathrm{deg} / 100 \mathrm{ft}$.
In the first case of the straight geothermal well, friction and wear of the centralizer bow is even on all sides. Therefore, the casing will remain centralized and centralizer heating and wear is not a problem.

The second case, of a geothermal well whose direction is changed frequently at a rate of $2 \mathrm{deg} / 100 \mathrm{ft}$, is a typical situation of direct interest. Frictional heating and wear rates for this condition will be estimated in following sections.

The third case of a highly deviated well is such that the centralizers as normally spaced do not prevent the casina from touching the side wall of the hole. Therefore, cementing problems are almost inevitable and so this case will not be examined herein. This situation is not considered to be a technical design problem of centralizer bow material, but rather a well operational problem. 


\subsubsection{Casing Centralizers Run in Geothermal Wells}

The purpose of this section is to estimate the effects of a casing centralizer bow run in a geothermal well which is deviated at approximately $2 \mathrm{deg} / 100 \mathrm{ft}$.

Friction Coefficient of Centralizer Bows on Casing or Open-Hole Side Walls

Search of the literature yielded little definitive data on the friction coefficients of centralizer bows on casing or open holes at elevated temperatures and in the presence of muds. For the ensuing analysis, these factors had to be estimated from other information. For instance, in predicting liner-hanger capacities, Brown Oil Tool, Inc. (69), uses a friction coefficient of 0.3 for downhole coefficients, although reference is made to past experiments giving coefficients of 0.78 for smooth ary surfaces, 0.11 for smooth surfaces with lard oil between, and 0.27 for surfaces with oxide films present. In an expression of frustration, the author says "all that we know is that the hanger will probably fail with a load somewhere between zero and the calculated load using a friction factor of one."

The Importance of Knowing What Happens to the Centralizer Bow

The centralizer bow is one of the most important mechanical aids in assuring a proper cementing job. When the drilled hole is straight and there is substantial clearance between the casing and the hole, cementing is straightforward and problems due to centralizing are not reported. Successful cementing has been reported in liner-hole combinations between a 7-in. 1 iner in an 8-5/8-in. hole $(\underline{70}, \underline{71})$. Shyrock (48) reports that "...this annular space may be reduced to $3 / 4$ in."

since it is unlikely that there is such a thing as a "straight" drilled hole, some amount of casing deviation is likely to occur, leading to side force on the centralizer bow. Figure 3-2 (subsection 3.1.2) has shown that a centralizer bow on a 7-in. casing to be run in a 9-1/2-in. hole will generate a side force greater than 1700 lb when displaced 1/4 in. from center. This 1/4-in. standoff represents 20 percent of the available standoff. With reference to Figure $3-8$, this deviation from central position will cause the flow around the casing to be asymmetric (see Figure 3-9). In fact, if the casing is displaced one-fourth of the available displacement distance, there will be no flow in the narrowest side at mud flow rates of $5 \mathrm{bbl} / \mathrm{min}(55)$. No flow in the narrow side will result in incomplete cementing which can lead to casing buckling and ultimately well abandonment. It should be noted that cementing rates of $5 \mathrm{bbl} / \mathrm{min}$ are typical (61). 

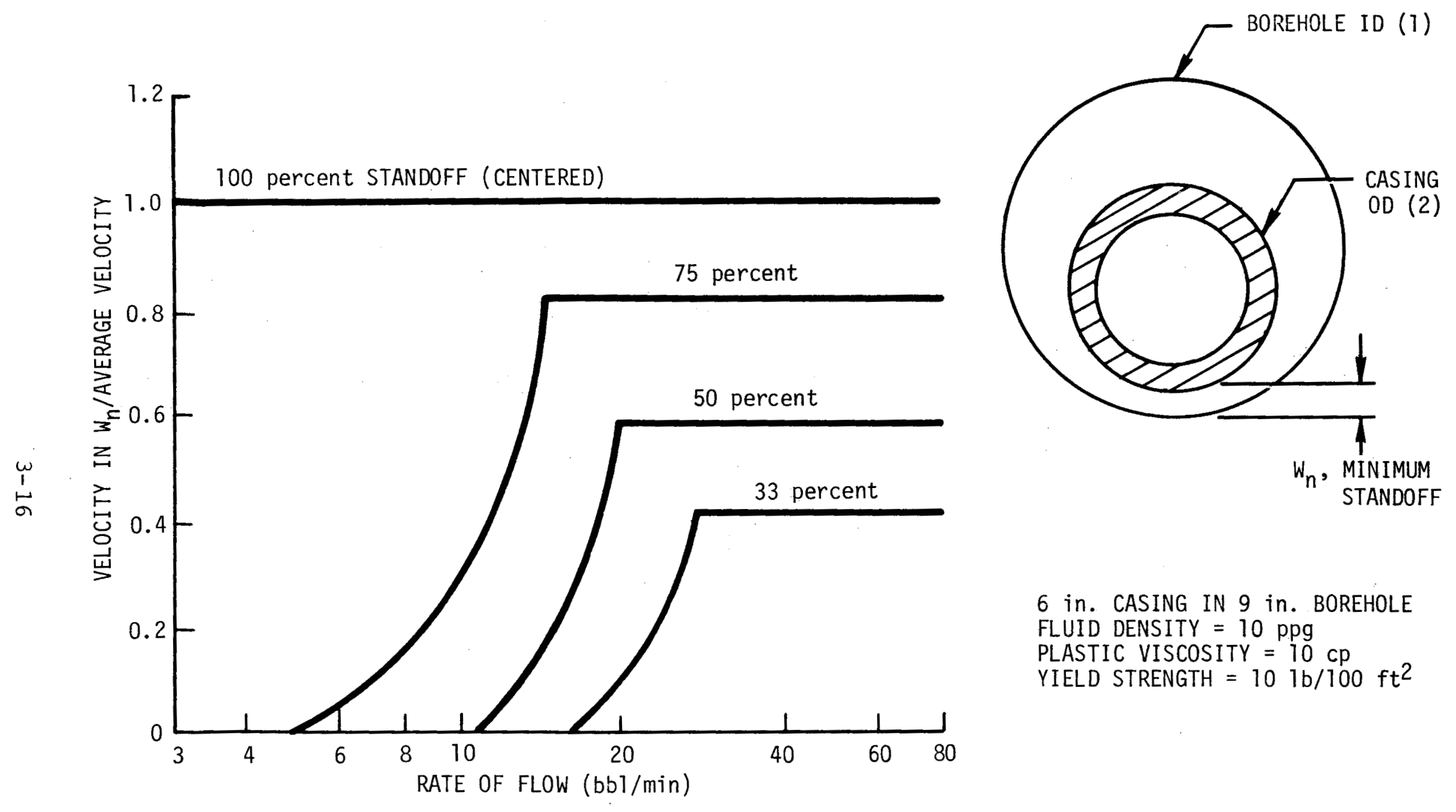

6 in. CASING IN 9 in. BOREHOLE

FLUID DENSITY $=10 \mathrm{ppg}$

PLASTIC VISCOSITY $=10 \mathrm{cP}$

YIELD STRENGTH $=101 \mathrm{~b} / 100 \mathrm{ft}^{2}$

FIGURE 3-8. - How decentralization affects velocity on the narrow side of the annulus in relation to overall rate of flow for one set of fluid and hole conditions (from Suman) (55). 


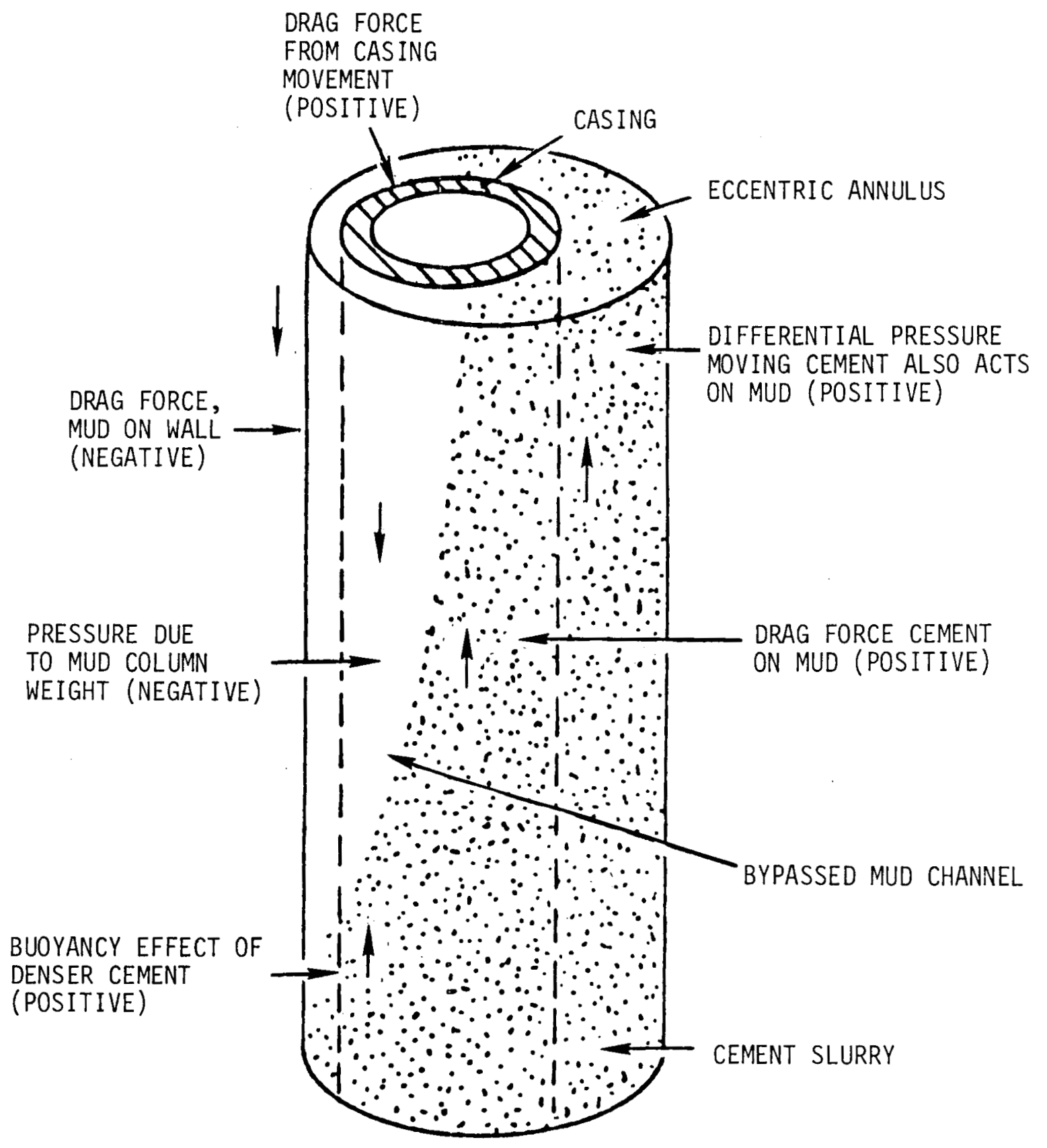

FIGURE 3-9. - Forces acting to displace, and resist displacement of a bypassed vertical mud column during primary cementing (from Suman) (5) . 
From the previous subsection we know that the friction coefficient of the centralizer bow on the hot casing, perhaps in the presence of mud, is not known. Yet it is apparent that very high forces are applied to the bows as they are slid downhole. If we assume that the bow side force is 800 lb at a friction coefficient of 0.7 for a distance of $5000 \mathrm{ft}$, there will be a total frictional work done equal to

$$
\text { Work }=(F)(\mu)(D)
$$

where

$$
\begin{aligned}
& F=\text { bow side force } \\
& \mu=\text { friction coefficient } \\
& D=\text { distance slid }
\end{aligned}
$$

Substituting

$$
\begin{aligned}
\text { Work } & =(800)(0.7)(5000) \\
& =2,800,000 \mathrm{ft}-1 \mathrm{~b} .
\end{aligned}
$$

This amount of work will result in both frictional heating of the bow and abrasive wear of the contact area, such that the centralizer bow may not be capable of supplying proper restoring forces downhole. It should be noted that API specification 10D is for centralizer bows at room temperature. No specification is made for capabilities of the spring steel at elevated temperatures. In addition, hardness of the centralizer bow is not specified. only bow restoring force is given. Testing according to the specification is to be accomplished at room temperature.

The question remains: what is the ability of centralizer bows to maintain their spring properties when they are slid downhole in a hot, mud-filled deviated geothermal well?

This question will be addressed by determining the temperature history of bow while it is slid into the hole. We can then determine the subsequent annealing and loss of strength of the bow steel. Two possible effects are: 1) reduction in hardness and subsequent increased wear; and 2) reduction in strength. Both can cause reduction in restoring force resulting in off center position of casing in the hole. 


\subsubsection{Thermal Analysis of the Centralizer Bow}

\section{Assumptions}

A thermal model of the centralizer bow was developed and solved numerically to determine the temperature-time history of the bow. The centralizer bow is assumed to slide downhole, under load in a mud-filled open hole.

The following assumptions are made:

a. Centralizer restoring force is 1600 lbf with a load on one member of a six-bow centralizer equal to $8001 \mathrm{bf}$

b. Sliding velocity is assumed to be 20 and $80 \mathrm{ft} / \mathrm{min}$ (this is one-half or two 40-ft casing sections per minute)

c. Coefficient of friction $=0.7$.

The centralizer bow is assumed to be 0.2 in. thick by $1-1 / 2$ in. wide (see Figure 3-4). Under load, a 2-in. section is assumed to contact the wall. A one-half bow model was used for the transient analysis and is shown in Figure 3-10.

Thermal Analysis

The heat input to the half-bow due to frictional heating is given as

$$
\dot{Q}_{\text {in }}=\frac{(0.25)(l)(\mu)(v)}{\left(778 \frac{\text { ft-1bf }}{\text { Btu }}\right)(60 \mathrm{sec} / \mathrm{min})}
$$

where

$$
\begin{aligned}
\dot{\mathrm{Q}}_{\text {in }} & =\text { heat flux (Btu/sec) } \\
\ell & =\text { applied load }(\mathrm{lbf}) \\
\mu & =\text { friction between bow and wall (assumed }=0.7 \text { ) } \\
V & =\text { centralizer velocity downhole ( } \mathrm{ft} / \mathrm{min}) \\
0.25 & =\text { assumed heat input to half-bow }
\end{aligned}
$$




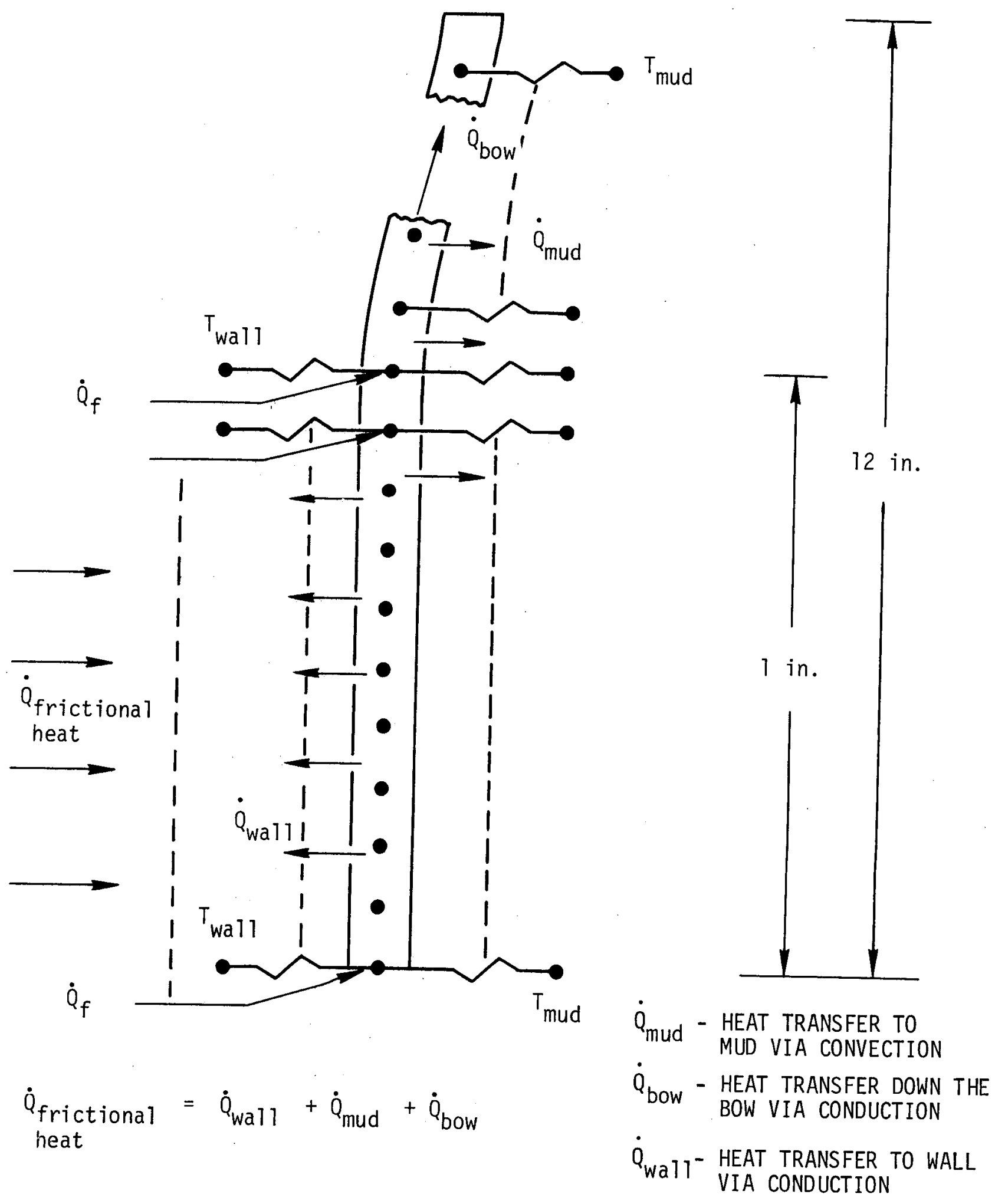

FIGURE 3-10. - Centralizer bow thermal model. 
For a constant velocity of $80 \mathrm{ft} / \mathrm{min}$, the heat flux is

$$
\begin{aligned}
\dot{Q}_{\text {in }} & =\frac{(0.25)(800)(0.7)(80)}{(778)(60)} \\
& =0.24 \mathrm{Btu} / \mathrm{sec}
\end{aligned}
$$

Note that the 0.25 factor is based on the assumption that one-half of the frictional heat input goes to the wall and one-half goes to the bow. Of the heat which goes to the bow, one-half will go to each half-bow.

We assume that the heat input is uniformly distributed across the 1-in. contact area. Conduction along the bow will be

$$
\dot{\mathrm{Q}}_{\mathrm{bow}}=\mathrm{kA} \frac{\partial \mathrm{T}}{\partial \mathrm{x}}
$$

where

$$
\begin{aligned}
\mathrm{k} & =\text { thermal conductivity of bow } \\
\mathrm{A} & =\text { cross sectional area of bow } \\
\frac{\partial \mathrm{T}}{\partial \mathrm{x}} & =\text { temperature gradient along bow }
\end{aligned}
$$

Conduction of heat to the wall can be given by

$$
\dot{Q}_{\mathrm{wall}}=\mathrm{h}_{\mathrm{w}} \mathrm{A}_{\mathrm{C}}\left(\mathrm{T}_{\mathrm{s}}-\mathrm{T}_{\mathrm{w}}\right)
$$

where

$$
\begin{aligned}
& \mathrm{h}_{\mathrm{W}}=\text { surface heat transfer coefficient } \\
& \mathrm{A}_{\mathrm{C}}=\text { contact area } \\
& \mathrm{T}_{\mathrm{S}}=\text { surface temperature } \\
& \mathrm{T}_{\mathrm{W}}=\text { wall temperature }
\end{aligned}
$$

Motion of the bow through the drilling mud will result in forced convection between the bow and the mud. Heat transfer to the mud will be given by

$$
\dot{\mathrm{Q}}_{\mathrm{mud}}=\mathrm{h}_{\mathrm{m}} \mathrm{A}_{\mathrm{s}}\left(\mathrm{T}_{\mathrm{s}}-\mathrm{T}_{\mathrm{m}}\right)
$$


where

$$
\begin{aligned}
& \mathrm{h}_{\mathrm{m}}=\text { surface heat transfer coefficient } \\
& \mathrm{A}_{\mathrm{s}}=\text { bow surface area exposed to mud } \\
& \mathrm{T}_{\mathrm{s}}=\text { bow surface temperature } \\
& \mathrm{T}_{\mathrm{m}}=\text { mud temperature }
\end{aligned}
$$

Thus, in the steady state, the frictional heat input to the bow is dissipated by conduction along the bow, conduction to the wall and convection to the surrounding drill mud. This can be written as

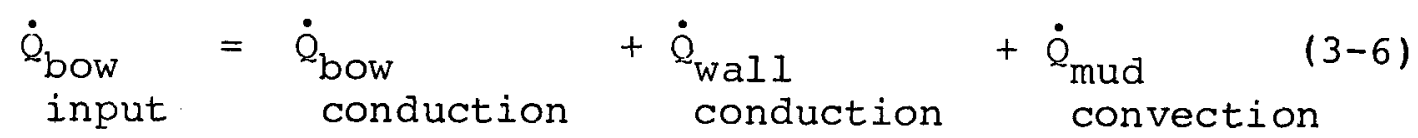

It was estimated that the heat transfer coefficient $h_{m}$ between the bow and the mud would be on the order of 10 to $100 \mathrm{Btu} / \mathrm{hr}$ $\mathrm{ft}^{2} \mathrm{OF}$. The value $\mathrm{h}=100$ would be approximately that for water on a vertical plate. Due to the thixotropic nature of the mud, the heat transfer coefficient $h_{m}$ of mud will be less than 100 .

The interface conductance between the bow and the wall is difficult to estimate due to the irregular nature of the interface. Interface conductance, for lightly loaded "rough" metal surfaces in contact with each other are on the order of $100 \mathrm{Btu} / \mathrm{hr}$ $\mathrm{ft}^{2} \mathrm{OF}$. Assuming a film thickness of mud of 0.01 in. between the bow and the wall gives a value of interface conductance equal to $180 \mathrm{Btu} / \mathrm{hr} \mathrm{ft}^{2} \mathrm{OF}$.

\section{Results of Thermal Analysis}

The results of the transient thermal analysis are shown in Figure 3-Il and are summarized in Table 3-2. It can be seen that the maximum temperature rise in the centralizer is a function of the rate at which the bow is run into the hole, the conductance between the bow and the wall, the wall temperature and the heat transfer between the bow and mud. Maximum temperature calculated for the centralizer bow was estimated to be in excess of $1200 \mathrm{~F}^{\circ}$ which is beyond the temperature necessary to anneal most heat treatable steels. Temperature rise of the bow is a function of heat transfer coefficient. An order of magnitude reduction in heat transfer coefficient between the bow and mud resulted in a $115^{\circ} \mathrm{F}$ decrease in maximum bow temperature, whereas a change in interface conductance of a factor of two resulted in a $225^{\circ} \mathrm{F}$ rise in bow temperature. 


\section{1}
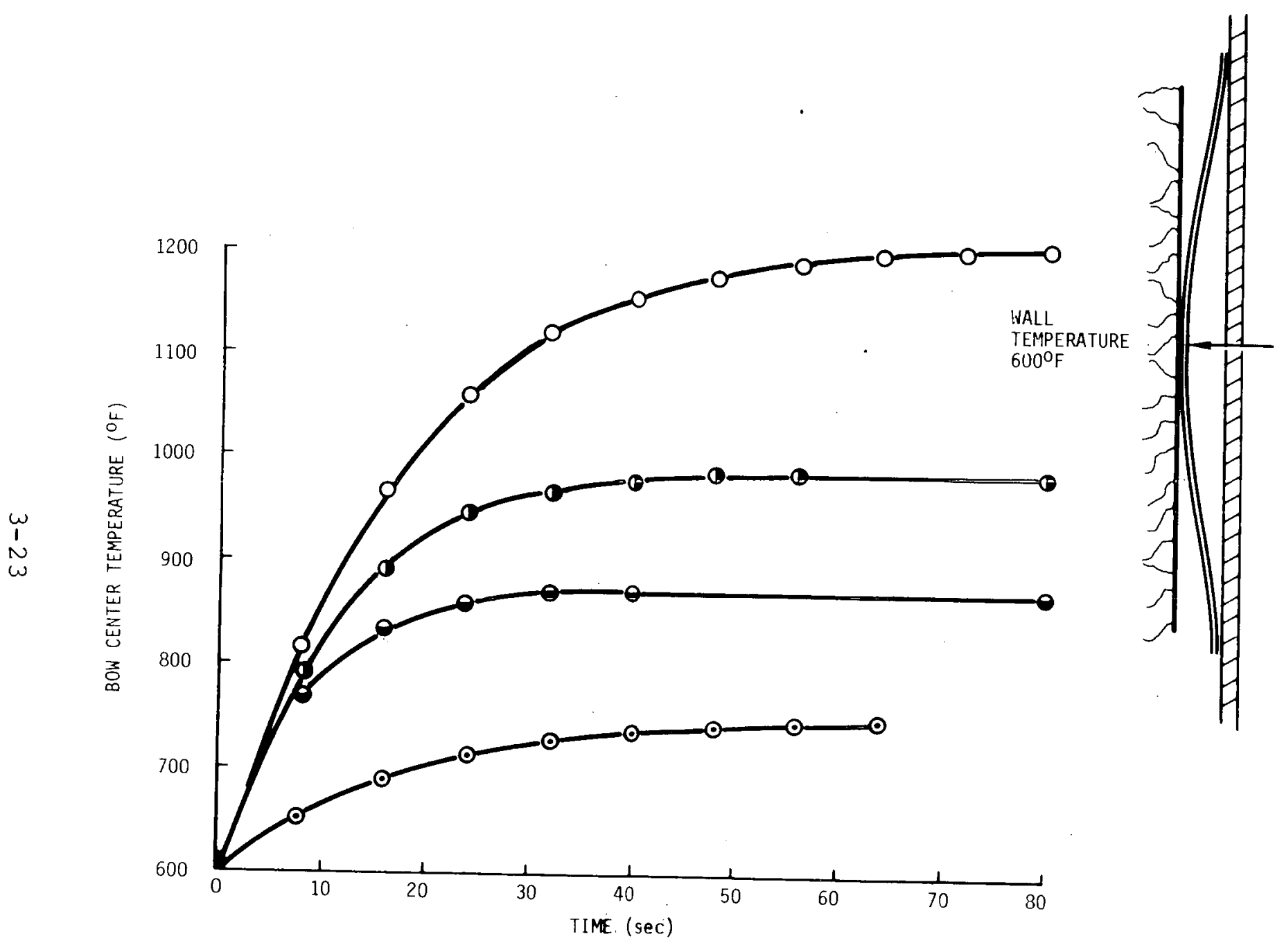

800 Ib RESTORING FORCE, $\mu=0.7$

$80 \mathrm{ft} / \mathrm{min}$ SLIDING VELOCITY CONDUCTANCE TO WALL $\mathrm{Btu} / \mathrm{hr} \mathrm{ft}^{2}$ of

$H=$ HEAT TRANSFER COEFFICIENT TO MUD Btu/hr $\mathrm{ft}^{2}$ OF

$\begin{array}{lll}O & C=90 & H=10 \\ \text { C } & C=180 & H=10\end{array}$

- $C=180 \quad H=100$

$20 \mathrm{ft} / \mathrm{min}$ SLIDING VELOCITY

○ $\mathrm{C}=90 \quad \mathrm{H}=10$

FIGURE 3-11. - Centralizer bow: transient temperature history at bow center. 
TABLE 3-2. - Temperature rise at midpoint of centralizer bow

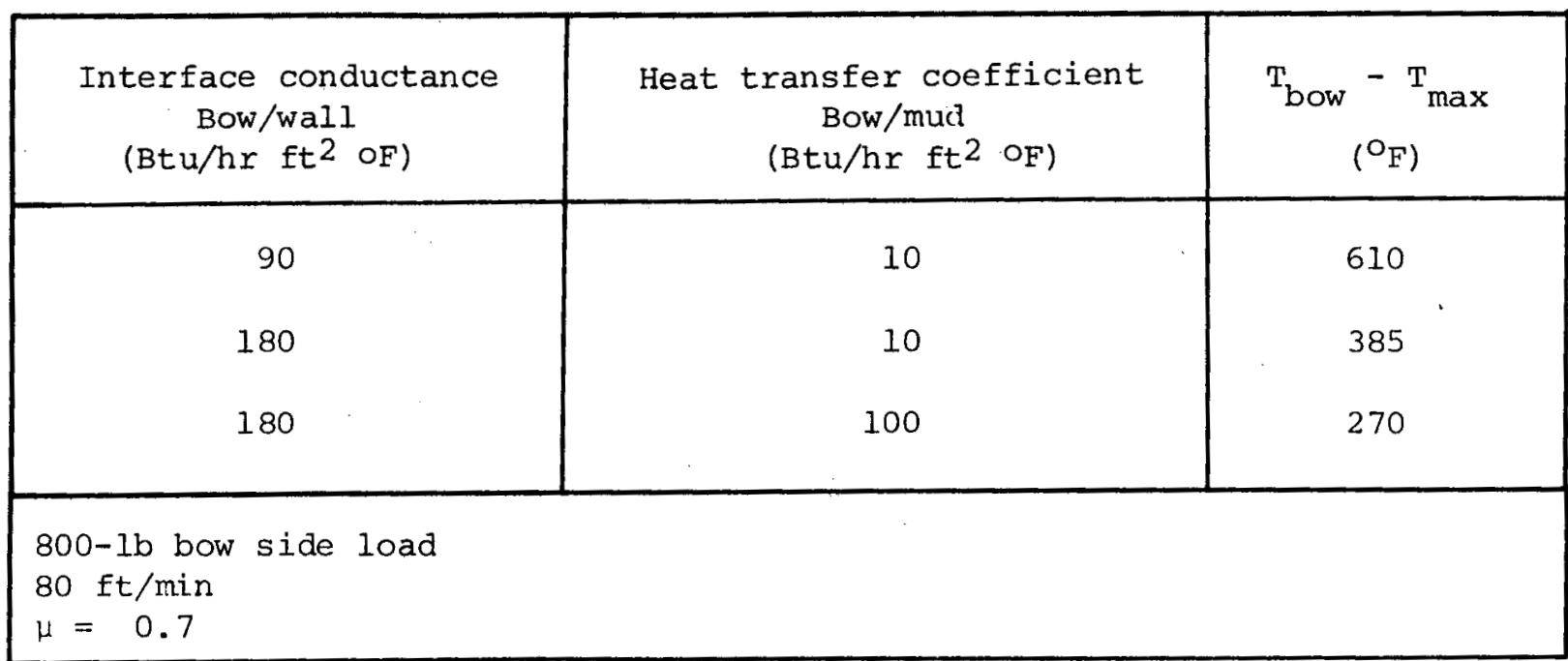

Slowing down the rate of heat input to the bow by sliding the centralizer downhole at slower velocities $(20 \mathrm{ft} / \mathrm{min})$ will reduce the rate of heat input and therefore the ultimate temperature rise of the bow.

Previous measurements of maximum casing temperatures where the casing had been subjected to drill string wear indicate that wear surfaces can reach $1650^{\circ}$ to $1900^{\circ} \mathrm{F}(72)$. While this information does not necessarily correlate with the centralizer bow problem, it does show that substantial elevated temperatures are possible with frictional heating in downhole drilling conditions. 


\subsubsection{Wear Analysis of the Centralizer Bow}

The Classical Theory

The classical theory of wear states that the volume of material removed by two surfaces rubbing against each other can be given by the relationship (66).

$$
V=\frac{k \ell x}{3 P}
$$

where

$$
\begin{aligned}
& \mathrm{V}=\text { volume lost } \\
& \mathrm{k}=\text { wear constant } \\
& \ell=\text { applied load } \\
& \mathrm{x}=\text { distance slid } \\
& \mathrm{P}=\text { material hardness }
\end{aligned}
$$

The wear constant $\mathrm{k}$ is determined essentially by experimentation. In general, its value will vary by a factor of two or more for materials tested against each other due to the difficulty of obtaining exact wear values and the variation of material properties.

There are two wear mechanisms of interest: adhesive and abrasive wear. Adhesive wear occurs when two smooth surfaces rub against each other and one is removed by adhesion to the other. Abrasive wear occurs when a rough hard surface or a soft surface containing hard particles slides on a softer surface and plows grooves into it. Two additional forms of wear, corrosive wear and fatigue wear, will not be addressed.

Estimate of Adhesive and Abrasive Centralizer Bow Wear

In estimating the wear of a centralizer bow on casing or open hole, we must be specific about the environment. If the centralizer bow is run on casing in a clean water environment, there is a high probability that the bow wear will be adhesive. If the bow is run over casing or open hole in the presence of sand-filled mud, wear of the centralizer bow will most likely be abrasive wear.

We can set limits on the centralizer bow wear by making both an adhesive wear and an abrasive wear calculation and then comparing these results to data obtained in the field. If we assume that the centralizer bow on soft casing is like low carbon steel on copper we can use $k=0.5 \times 10^{-3}$ (67) (see Table 3-3). 
TABLE 3-3. - Wear constant $k$ of various sliding combinations

\begin{tabular}{|l|c|}
\hline \multicolumn{1}{|c|}{ Combination } & Wear Constant $\mathrm{k} \times 10^{3}$ \\
\hline Zinc on zinc & 160.00 \\
Low carbon steel on low carbon steel & 45.00 \\
Copper on copper & 32.00 \\
Stainless steel on stainless steel & 21.00 \\
Copper (on low carbon steel) & 1.50 \\
Low carbon steel (on copper) & 0.50 \\
Bakelite on bakelite & 0.02 \\
\hline
\end{tabular}

This would be for unlubricated adhesive wear of the centralizer bow. If we assume that the centralizer hardness is $425 \mathrm{~kg} / \mathrm{mm}^{2}$, as derived from reference (60), the side load is $800 \mathrm{lb}$, and the distance slid is $5000 \mathrm{ft}$, we calculate the volume lost as

$$
\begin{aligned}
& \mathrm{V}=\frac{(0.0005)(5000 \mathrm{ft} \times 12 \mathrm{in} . / \mathrm{ft})(800 \mathrm{lb})}{(3)\left(425 \frac{\mathrm{kg}}{\mathrm{mm}^{2}}\right)\left(2.2 \frac{\mathrm{lb}}{\mathrm{kg}}\right)\left(25 \frac{\mathrm{mm}}{\mathrm{in}}\right)^{2}} \\
& V_{\text {adhesive }}=0.0137 \mathrm{in}^{3}
\end{aligned}
$$

If, on the other hand, the centralizer bow slides over abrasive rock in the presence of sand-filled mud, we would expect that the wear would be abrasive. With reference to Table 3-4 from Rabinowicz (67) we see that the maximum two-body abrasive wear constant would be $\mathrm{k}=180 \times 10^{-3}$. Substituting this into abrasive Equation 3-7, we calculate the volume of material lost as

$$
\begin{aligned}
& \mathrm{V}=\frac{(0.180)(5000 \mathrm{ft} \times 12 \mathrm{in} . / \mathrm{ft})(800 \mathrm{lb})}{(3)\left(425 \frac{\mathrm{kg}}{\mathrm{mm}^{2}}\right)\left(2.2 \frac{\mathrm{lb}}{\mathrm{kg}}\right)\left(25 \frac{\mathrm{mm}}{\mathrm{in} .}\right)^{2}} \\
& \mathrm{~V}_{\text {abrasive }}=4.93 \mathrm{in.}
\end{aligned}
$$


TABLE 3-4. - Abrasive wear constants k

\begin{tabular}{lcccc}
\multicolumn{1}{c}{ Investigator } & wear Type & Size $(\mu)$ & Materials & $\mathrm{k}\left(\times 10^{+3}\right.$ ) \\
Spurr, et al. (1957) & 2-body & - & Many & 180.0 \\
Spurr, et al. (1957) & 2-body & 110 & Many & 150.0 \\
Avient, et al. (1960) & 2-body & 40 to 150 & Many & 120.0 \\
Lopa (1956) & 2-body & 260 & Steel & 80.0 \\
Kruschov, et al. (1958) & 2-body & 80 & Many & 24.0 \\
Samuels (1956) & 2-body & 70 & Brass & 16.0 \\
Toporov (1958) & 3-body & 150 & Steel & 6.0 \\
Rabinowicz, et al. (1961a) & 3-body & 80 & Steel & 4.5 \\
Rabinowicz, et al. (1961b) & 3-body & 40 & Many & 2.0
\end{tabular}

Obviously, this volume of material loss on a centralizer bow, 1.5 in. wide, contacting a 2 -in. long section would destroy the 0.2-in. thick bow. In fact, a bow thickness of 1.64 in. thick would be worn through due to abrasive wear.

Reports of centralizer bow wear leading to cementing problems do not exist in the literature, probably due to the fact that cemented bows are never recovered and manufacturers do not admit to equipment problems in the open literature. Based on the above analysis, we would conclude that if the centralizer bow is subjected to a clean hole and adhesive wear, there probably is no loss of properties due to wear. If, on the other hand, the centralizer bow is subjected to abrasive wear, obviously the bow would fail, according to the above analysis. Personal communication with manufacturers has revealed that centralizer bows used on wireline instruments have been recovered and were severely worn $(73,24)$. Unfortunately, these bows have not been saved nor have any tests been made to determine reduction in restoring force of these centralizer bows. More detailed information is required to determine if centralizer bow wear can lead to potential cementing problems.

Evidence of Wear Data for Environments of Interest

Casing wear has been measured and reported by Bradley and Fontenot (75). Based on extensive laboratory studies of drill pipe tripping wear tests and wireline wear tests on casing, wear coefficients were reported for a variety of simulated downhole conditions. These wear measurements were made between drill pipe and wireline and both K55 and P110 casing. The wear constants $C_{W t}$ and $C_{W W}$ were related to the volume of material removed, drili pipe or wireline contact force, and distance travelled, according to the equation 


$$
\mathrm{CW}=\frac{\mathrm{Vt}}{\mathrm{FL}}
$$

Where

$$
\begin{aligned}
& \mathrm{Vt}=\text { volume removed } \\
& \mathrm{F}=\text { contact force } \\
& \mathrm{L}=\text { distance travelled across the wear point }
\end{aligned}
$$

This expression can be directly related to the classical theory of friction and wear by substituting Equation 3-8 into Equation 3-7 to obtain the expression

$$
\mathrm{k}=3 \mathrm{C}_{\mathrm{w}} \mathrm{P}
$$

where $k, C_{w}$ and $P$ have been defined before.

In reducing the drill pipe and wireline wear data, it was necessary to estimate the hardness $P$ of both the drill pipe and the wireline since these data were not given. Typical drill pipe would be hardened steel to at least $\mathrm{RC} 55$. With reference to the Metals Handbook, reference $(60)$, we can translate this hardness to the Knoop hardness for drill pipe as $520 \mathrm{~kg} / \mathrm{mm}^{2}$. For the wireline, data are not available. However, since wireline must remain ductile, such steel would be hardened to approximately RC40. This hardness translates to a Knoop hardness for wireline of $400 \mathrm{~kg} / \mathrm{mm}^{2}$.

By taking the data obtained by Bradley and Fontenot and reducing it to a dimensionless wear coefficient we can determine the mechanism of wear for the centralizer bow. In Tables 3-5 and 3-6, the drill pipe tripping wear on casing and wireline wear on casing have been appropriately reduced and tabulated versus the environment. A summary of the mean wear constant for the various test conditions has been reported in Table 3-7. Please note that the wear "constant" $k$ for any given test condition can vary widely.

\section{Centralizer Bow Wear Versus the Environment}

Based on the wear constants derived from Bradley and Fontenot's data, we can conclude that the geothermal environment will result in both adhesive and abrasive wear. Drill pipe wear on casing in the presence of weighted water-based mud with 2 to 8 percent drill solids produced a wear constant

$k=3.2 \times 10^{-3}$. Drill pipe wear on casing in the presence of 
TABLE 3-5. - Wear constant from drill pipe tripping wear on casing from Bradley and Fontenot (75)

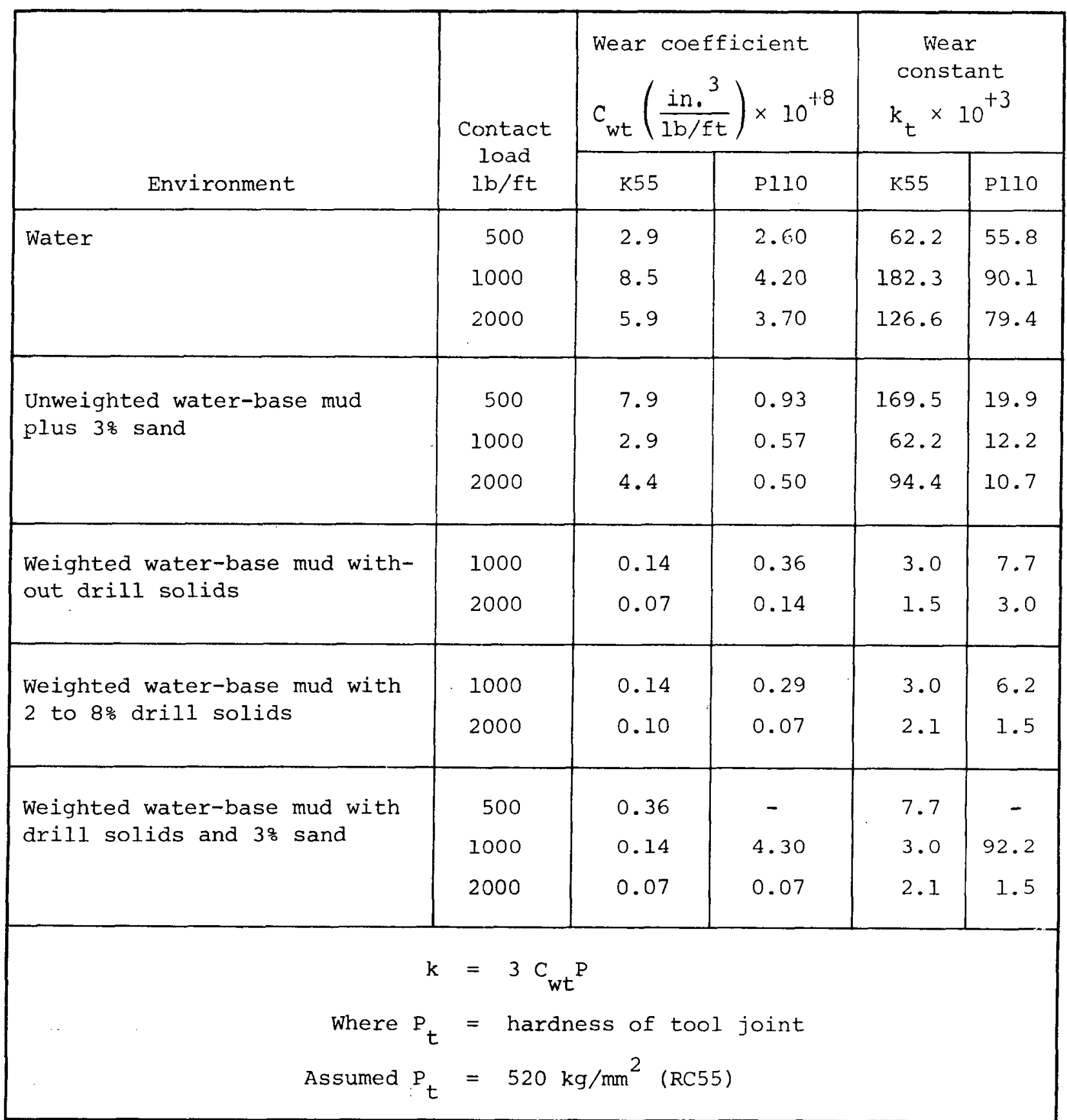




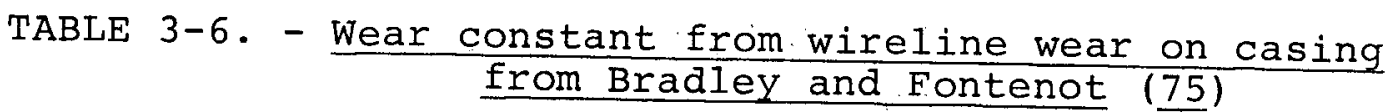

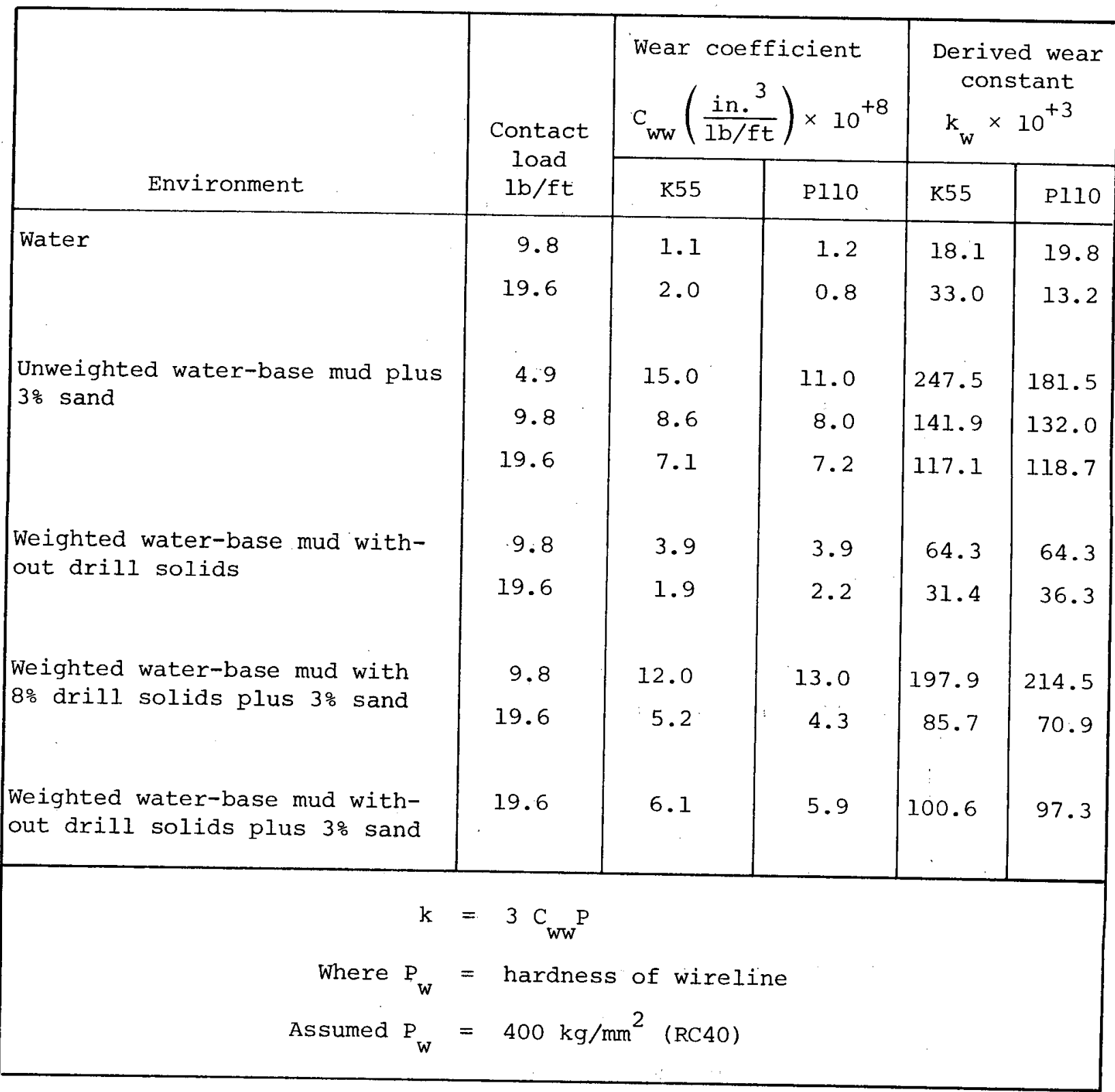



TABLE 3-7. - Summary of wear constant data for drill pipe

\begin{tabular}{|c|c|c|}
\hline Environment & wear type & $\begin{array}{c}\text { Mean } \\
\text { wear } \\
\text { constant } \\
\mathrm{k} \times 10^{+3}\end{array}$ \\
\hline Water & $\begin{array}{l}\text { Drill pipe on casing } \\
\text { Wireline on casing }\end{array}$ & $\begin{array}{l}99.4 \\
21.0\end{array}$ \\
\hline $\begin{array}{l}\text { Unweighted water- } \\
\text { base mud plus } 3 \% \\
\text { sand }\end{array}$ & $\begin{array}{l}\text { Drill pipe on casing } \\
\text { wireline on casing }\end{array}$ & $\begin{array}{r}61.5 \\
156.4\end{array}$ \\
\hline $\begin{array}{l}\text { Weighted water- } \\
\text { base mud without } \\
\text { drill solids }\end{array}$ & $\begin{array}{l}\text { Drill pipe on casing } \\
\text { Wireline on casing }\end{array}$ & $\begin{array}{r}3.8 \\
49.1\end{array}$ \\
\hline $\begin{array}{l}\text { Weighted water- } \\
\text { base mud with } 2 \\
\text { to } 8 \% \text { drill solids }\end{array}$ & $\begin{array}{l}\text { Drill pipe on casing } \\
\text { Wireline on casing }\end{array}$ & $\begin{array}{l}3.2 \\
-\end{array}$ \\
\hline $\begin{array}{l}\text { Weighted water- } \\
\text { base mud with } \\
\text { drill solids and } \\
3 \% \text { sand }\end{array}$ & $\begin{array}{l}\text { Drill pipe on casing } \\
\text { Wireline on casing }\end{array}$ & $\begin{array}{l}21.3 \\
98.9\end{array}$ \\
\hline $\begin{array}{l}\text { Weighted water- } \\
\text { base mud with } 8 \% \\
\text { drill solids plus } \\
3 \% \text { sand }\end{array}$ & Wireline on casing & 142.3 \\
\hline
\end{tabular}


water produced a wear constant of $99.4 \times 10^{-3}$, some 30 times the wear constant for water-based mud. The wireline wear on casing in the presence of sand gives a wear constant

$\mathrm{k}=156.4 \times 10^{-3}$. Following the condition used previously, we would estimate the centralizer bow wear as shown in Table 3-8.

Results of the Wear Analysis

Based on the classical theory of wear, we are able to estimate the amount of material removed for both adhesive wear and abrasive wear conditions of a centralizer bow on casing or open hole. For adhesive wear, corresponding to centralizer bow on casing in the presence of weighted water-base mud, we estimate that there will be little or no wear problem for the assumed conditions. For abrasive wear, corresponding to centralizer bow on casing or open hole in unweighted water-base mud with 3 percent sand, we estimate that there will be severe wear of the centralizer bow, probably leading to centralizer bow failure and probable cementing problems.

TABLE 3-8. - Probable centralizer bow wear 800-1b side force, $5000-f t$ travel

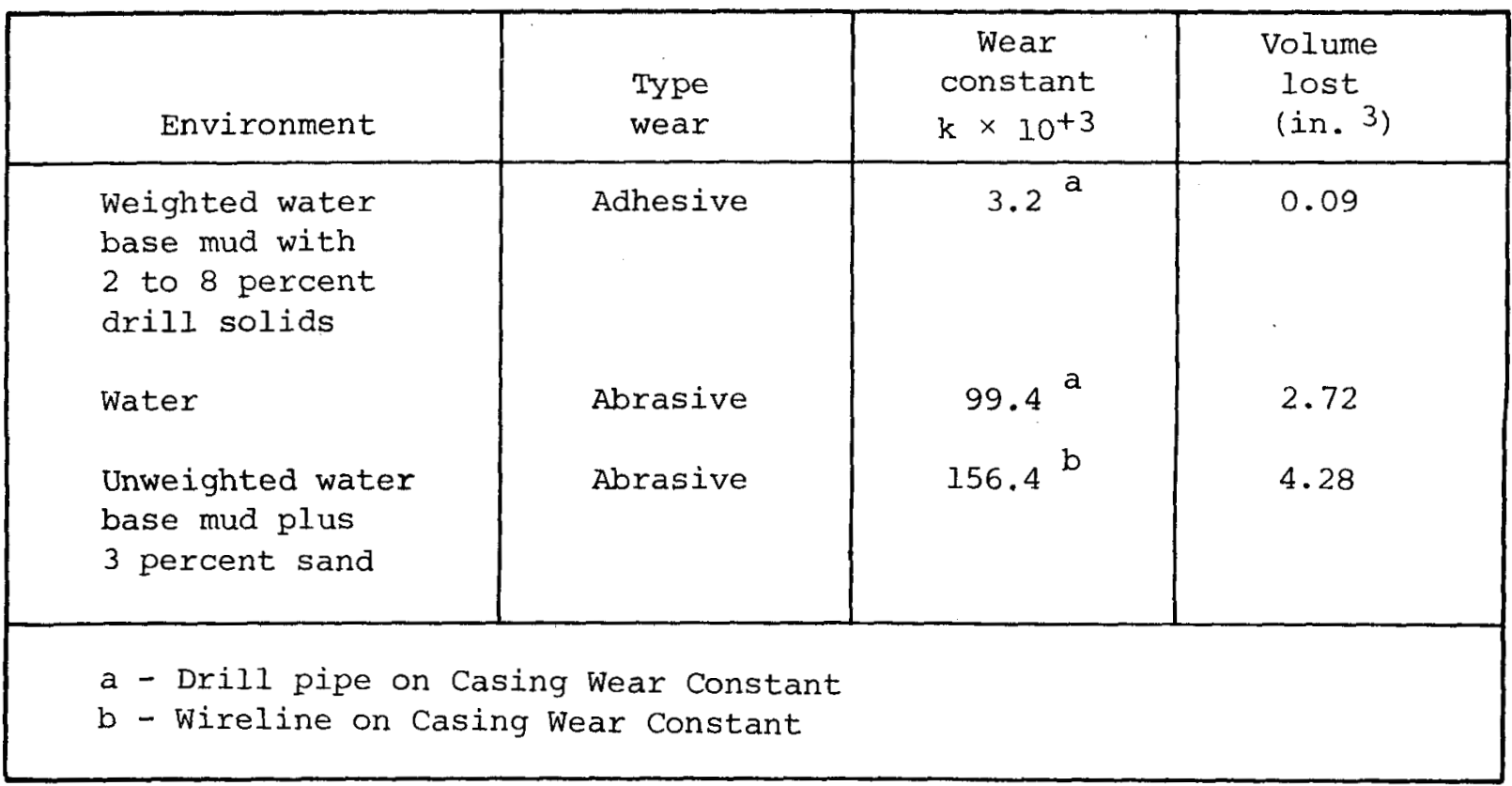


Reduction of Restoring Force due to Centralizer Bow Wear

Having estimated the volume of spring bow material removed by achesive and abrasive wear, the next step is to determine the effect of this removal on the ability of the spring bow to centralize. We shall examine three models of the spring bow, as follows:

- Centralizer bow - no wear

- Centralizer bow - worn through in center

- Centralizer bow - worn half-way through center.

Centralizer Bow - No Wear

Although centralizer bows are curved, for the purpose of analysis we shall treat them as a straight spring, clamped at both ends. The engineering model is shown in Figure 3-1.2.

Deflection of the clamped spring at the center is given as

$$
\begin{aligned}
& Y=-\frac{I}{192} \frac{F L^{3}}{E I} \\
& I=\frac{b h^{3}}{12}
\end{aligned}
$$

where

$$
\begin{aligned}
& \mathrm{F}=\text { applied load } \\
& \mathrm{L}=\text { spring length } \\
& \mathrm{E}=\text { Modulus of Elasticity } \\
& \mathrm{I}=\text { moment of inertia }
\end{aligned}
$$
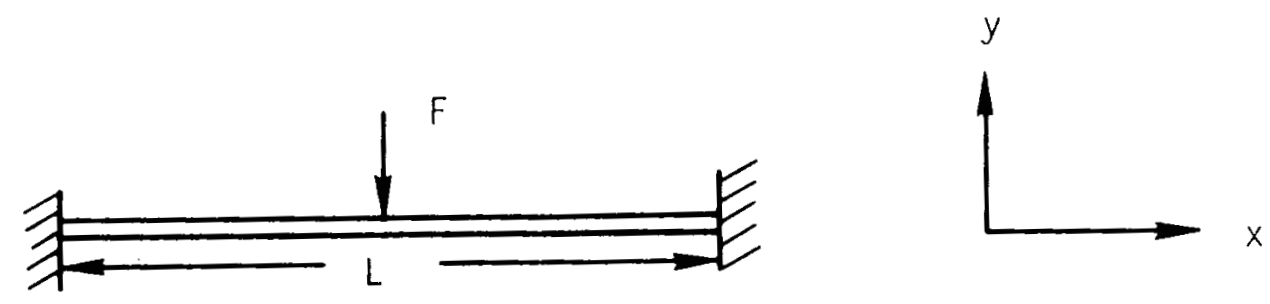

FIGURE 3-12. - Centralizer bow spring model - no wear. 
Equation 10 is a classical clamped beam solution which can be found in numerous handbooks ( $\underline{65})$.

\section{Centralizer Bow - Worn through in Center}

We shall again assume that the centralizer bow is clamped at both ends but is split in the middle. Thus, one-half of the applied load will be concentrated at the end of one-half of the clamped beam (see Figure 3-13).

Deflection of the split spring at the center will be

$$
y=-\frac{1}{3} \frac{f \ell^{3}}{E I}
$$

where

$$
\begin{aligned}
& f=F / 2 \\
& \ell=L / 2
\end{aligned}
$$

Therefore, in terms of the total load and bow length, the deflection may be written as

$$
\mathrm{Y}=-\frac{1}{48} \frac{\mathrm{FL}^{3}}{\mathrm{EI}}
$$

From this simple analysis, we can conclude that for a spring worn completely through at the center, the restoring force of the spring will be reduced by a factor of four as compared to the virgin spring.

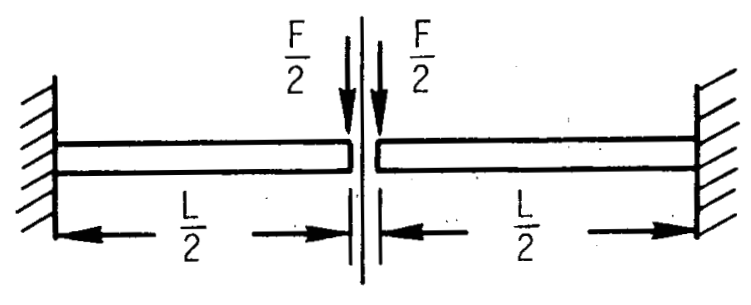

FIGURE 3-13. - Centralizer bow spring model - worn through. 
Centralizer Bow - Worn Half-Way through Center

Since the previous case is the extreme, we want to determine what happens to the spring when one-half of the material is removed at the center of the spring. Obviously, the degraded spring will exert a restoring force somewhere between the unworn, virgin spring and the broken spring.

We treat the worn spring in the center as a short beam which supplies a restoring moment such that the slope of the beam in the center is zero. Assume that the half bow spring model has a 1-in. long section of worn material at the end (see Figure 3-14).

If the moment of inertia of the original beam is $I$, then the moment of inertia of the 50 percent worn beam will be $\mathrm{I} / 8$.

At the interface between the full beam and the worn section, the slope of the beams will be the same. Thus for the beam

$$
\theta=\frac{1}{2} \frac{E \ell^{2}}{E I}-\frac{M_{O} l}{E I}
$$

For the short section

$$
\theta=\frac{\mathrm{M}_{0} l_{s}}{E I_{S}}
$$

where

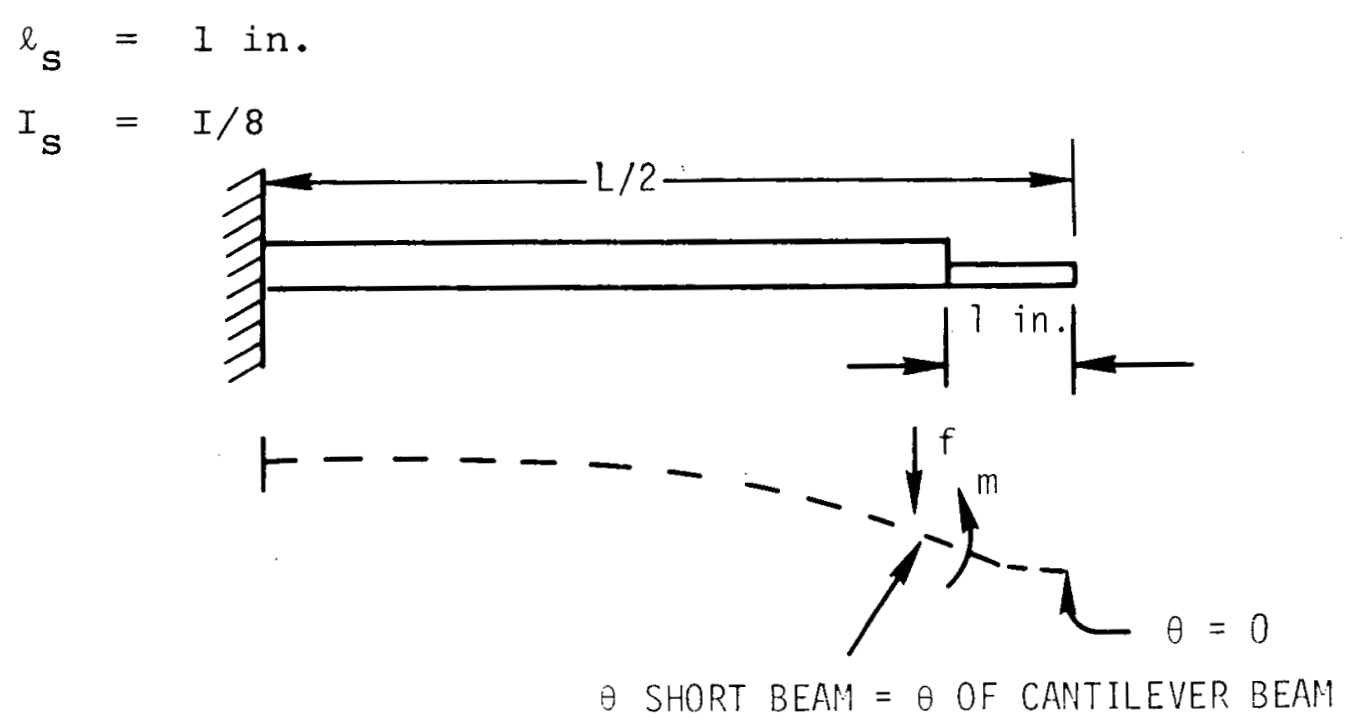

FIGURE 3-14. - Centralizer bow spring model - 50 percent worn. 
Solving for $\theta$ we get

$$
\theta=\frac{1}{2} \frac{f \ell^{2}}{E I}-\theta\left(\frac{l}{l_{s}}\right)
$$

or

$$
\theta=\left(\frac{8 l_{s}}{8 l_{s}+l}\right)\left(\frac{1}{2}\right)\left(\frac{f l^{2}}{E I}\right)
$$

If we assume that the short length, $\ell_{s}$, is $I$ in. long and the half spring length, $\ell$, is $7 \mathrm{in.}$ long, we get

$$
\theta=\left(\frac{8}{15}\right)\left(\begin{array}{ll}
\frac{1}{2} & \frac{\mathrm{E} \ell^{2}}{\mathrm{EI}}
\end{array}\right)
$$

We conclude from this simple model that the effect of a halfworn spring, assuming the conditions chosen, is to reduce the deflection of the cantilever spring by a factor of $8 / 15$ as compared to the cantilever model.

Restoring Force for Unworn, Broken and Partially Worn Centralizer Bows

Next we can calculate the amount of restoring force supplied by the three spring combinations for a given deflection. Deflection of the unworn spring and the broken or parted spring is given by Equations 3-10 and 3-13, repectively. We can solve for the effective spring constant as

$$
\mathrm{k}_{\substack{\text { clamped } \\ \text { spring }}}=\left|\frac{\mathrm{F}}{\mathrm{Y}}\right|=192 \frac{\mathrm{EI}}{\mathrm{L}^{3}}
$$

and

$$
\begin{gathered}
\mathrm{k}_{\mathrm{b} \text { roken }}^{\text {spring }} \\
\text { spring }
\end{gathered} \mid=48 \frac{\mathrm{EI}}{\mathrm{L}}
$$


Deflection of the half-worn spring will be given by

$$
y=\frac{F L^{3}}{48 E I}-\frac{1}{8} \frac{M L^{2}}{E I}
$$

From Equations $3-14$ and $3-15$ we can solve for $M_{O}$ in terms of $F$. We get the following

$$
M_{0}=\frac{l^{2}}{l_{s}} \quad \frac{F}{60}
$$

Substituting Equation 3-22 into 3-21, we get

$$
y=\frac{F L^{3}}{48 \mathrm{EI}}\left(1-\frac{1}{40} \frac{I}{l_{s}}\right)
$$

Thus, the spring constant is

$$
\mathrm{k}_{\substack{\text { worn } \\ \text { spring }}}=\left|\frac{F}{y}\right|=\frac{48 \mathrm{EI}}{L^{3}}\left(\frac{40 l_{s}}{40 l_{s}-I}\right)
$$

If we now substitute Equation $3-20$ into $3-21$, we find

$$
k_{\text {worn }}^{\text {spring }}=\left(\frac{40 l_{s}}{40 l_{s}-I}\right) \quad \begin{aligned}
& k_{\text {broken }} \\
& \text { spring }
\end{aligned}
$$

For the chosen model, $L=14 l_{\mathrm{S}^{\circ}}$

Therefore

$$
\begin{aligned}
& \mathrm{k}_{\text {worn }}=1.54 \mathrm{k}_{\text {broken }} \\
& \text { spring spring }
\end{aligned}
$$

or

$$
\begin{aligned}
& \mathrm{k}_{\text {worn }}=0.38 \mathrm{k} \text { clamped } \\
& \text { spring }
\end{aligned}
$$


Based on this analysis we conclude that the 50 percent worn spring is 54 percent stronger than a broken spring. In addition, we conclude that the 50 percent worn spring has lost 62 percent of its initial strength. This conclusion applies up to the point where the central web remains elastic. As soon as the central web yields the conclusions differ.

The worn spring will only be able to support a maximum bending moment at the worn element. This bending moment will be determined by the relationship

$$
M_{\max }=\frac{\sigma I_{S}}{c}
$$

where

$$
\begin{aligned}
& \sigma=\text { material yield strength } \\
& \mathbf{c}=\text { half thickness of worn bow }
\end{aligned}
$$

If we assume that the bow material yield strength is $80,0001 \mathrm{~b} / \mathrm{in} .^{2}$ and the worn half thickness is 0.05 in., we get a maximum moment,

$$
\mathrm{M}_{\max }=200 \mathrm{in} \cdot 1 \mathrm{bf}
$$

Past the point of yield, the deflection will be given as

$$
y=\frac{F I^{3}}{48 E I}-\frac{1}{2} \frac{{ }^{M} O_{\max } \ell^{2}}{E I}
$$

For the three models we can plot the bow deflection versus restoring force, recognizing that the worn bow will yield at a maximum bending moment. The results are plotted in Figure 3-15.

\section{Casing Displacement due to Worn Centralizer Bow}

From Figure 3-15 we find that the half-worn centralizer bow model will support approximately 200 lbf before yielding. Past this load the centralizer bow acts essentially as two broken springs cantilevered from opposite ends. At a typical bow slide force of 500 lbf, the bow deflection of the worn spring will be 0.77 in. The matching unworn centralizer bow spring on the opposing side of the casing will support 500 lbf at a defection 
(1)

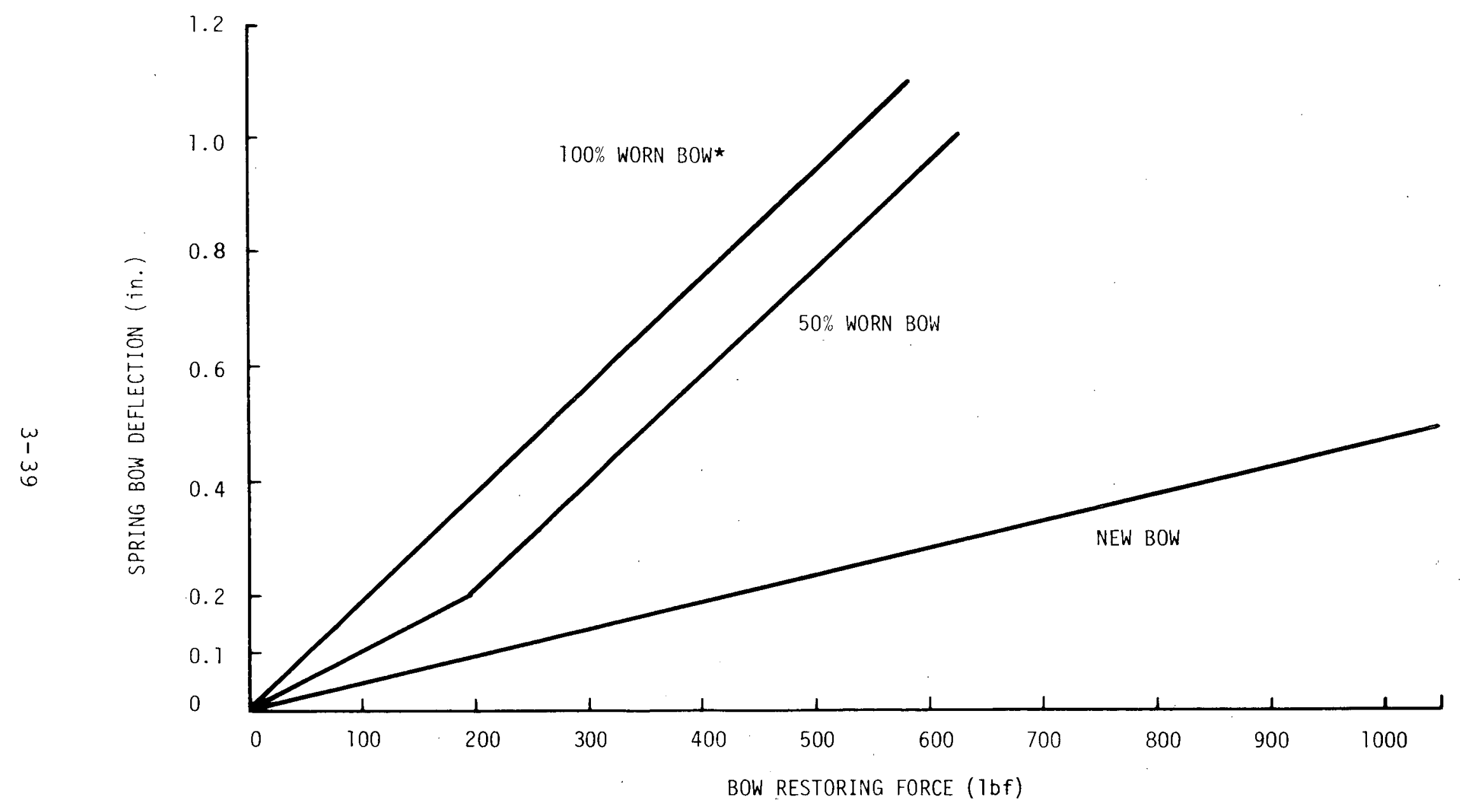

*SPLIT IN CENTER

FIGURE 3-15. - Centralizer bow deflection. 
of 0.24 in. Thus, if the centralizer bow had located a casing uniformly $0.5 \mathrm{in}$. from the casing wall, the casing would shift one-half of the available distance due to the half-worn spring.

\subsubsection{Conclusions}

Frictional heating of centralizer bows will depend Iinearly on the bow side load, the friction coefficient between the bow and wall, the contact area, the wall temperature, the thermal conductance to the wall and mud and the velocity of insertion of the centralized case into the hole. In the worst case of rapid centralizer bow insertion ( $80 \mathrm{ft} / \mathrm{min}$ ) into a deviated hole $(2.5 \mathrm{deg} / 100 \mathrm{ft}$ ) with a side load on a single-bow of $800 \mathrm{lb}$ and a friction coefficient between the bow and wall equal to 0.7 , the bow temperature will rapidly rise to a temperature sufficient to anneal the metal, leading to a loss of side force to centralize the bow. In the best case, where the centralizer bow is slowly inserted into the hole $(20 \mathrm{ft} / \mathrm{min})$, side load due to hole deviation is low, or the friction coefficient is low, thermal rise of the bow will be only slightly above ambient temperature and the bow will retain its spring properties.

Wear between the centralizer bow and the casing or hard open hole will depend greatly on the medium in which the centralizer bow is used. If the centralizer bow is slid downhole in weighted water-base muds, the volume lost due to wear of the centralizer bow should be minimal. If the centralizer bow is slid downhole over sand-impregnated mud, the centralizer bow may be subjected to severe wear and failure. Wear will be proportional to the side load on the centralizer bow such that hole deviation, casing string tension, buoyancy of the casing, etc., will play a very important role in the centralizer bow problem.

Frictional heating of a centralizer bow may lead to annealing of the spring steel which will lead to reduced yield strength of the spring. Frictional wear of a centralizer bow spring will greatly reduce the ability of the worn spring to centralize a casing. Loss of restoring force by the worn spring sharply increases once the spring centralizer bow yields at the point of contact. The inability of the centralizer bow to centralize will lead to incomplete cementing of the "centralized" casing. 


\subsubsection{Recommendations}

While little is actually known about the problem of frictional heating and wear of centralizer bows on casing or open hole completions, there is sufficient evidence to believe that centralizer bows may fail in normally, deviated holes. Failure of the centralizer bow would be measured in its inability to centralize the casing so that incomplete cementing took place.

Based on the analysis in this report, the author recommends that tests be conducted to establish the friction and heat transfer coefficients between centralizer bows and mud covered casing and/or open hole environments. Based upon these data, and analyses, open hole tests should be conducted to measure the thermal response and wear of centralizer bows in select geothermal environments. Tests can be conducted to determine the loss of centralizer bow effectiveness with frictional heating and wear.

If it is proven that frictional heating and wear of centralizer bows is a problem in geothermal completions, steps can be taken to improve the product such that centralizer bow failure can be prevented. Failure of centralizer bows to centralize casing can lead to geothermal well failure. It seems prudent to assure that well failure does not take place because of a faulty component.

3.2 Geothermal Well Cementing Plug Failure Due to Frictional Heating and Wear

\subsubsection{Introduction}

In order to cement a casing into a geothermal well, special techniques are used to assure that the cement fills the annular space surrounding the casing. This cement sheath must protect the casing from corrosion by thermal brines and gases and prevent flow of the geothermal water and steam outside the casing.

In a typical application, primary cementing is begun by using a float collar at a specified distance above the cement guide shoe (Figure 3-16). An excess of cement is pumped downhole between two cementing plugs whose function is to separate the cement from the mud ahead of the bottom plug and behind the top plug. This cement volume can easily exceed the volume required by 50 percent (Figure 3-17).

Shryock and Smith ( $\underline{48)}$ recommend that specific steps be taken to assure that the volume of cement is isolated from the mud and to assure that the annular space to be cemented is clear of loose mud cake. The authors recommend that a viscous spacer be used ahead of the cement. Viscosity should be such that the spacer is more viscous than the mud to be cleared from the annulus but less viscous than the cement. The viscous spacer is to be followed by $10 \mathrm{bbl}$ of water and then by 20 to $50 \mathrm{bbl}$ of a hole 


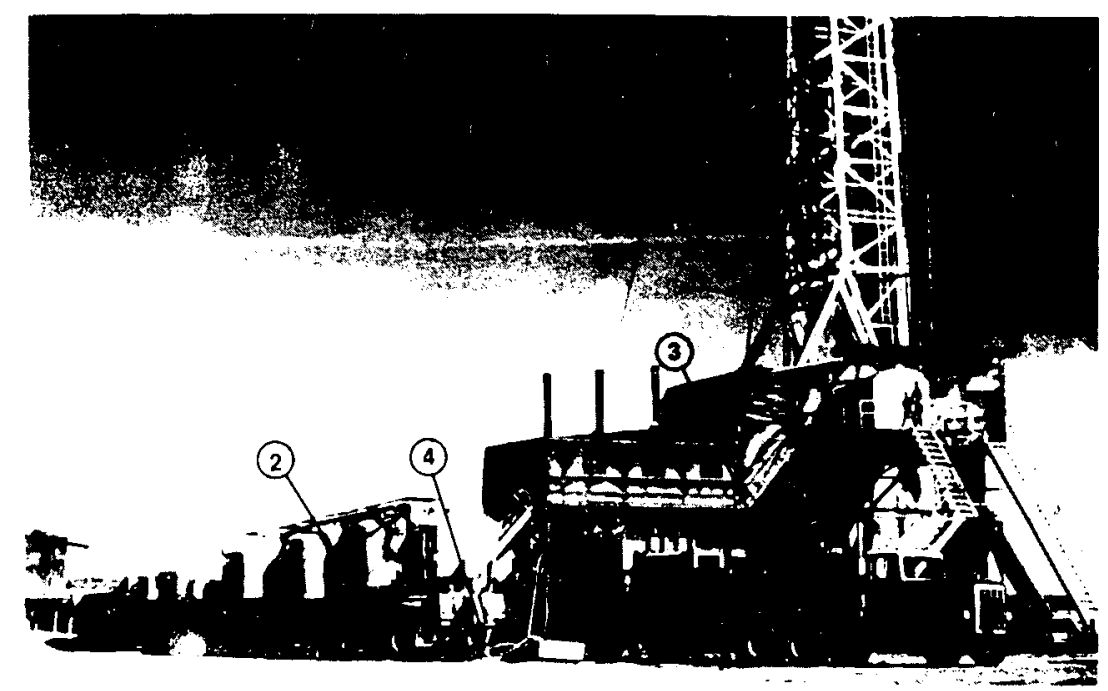

1 - PLUG CONTAINER

2 - BULK MATERIAL STORAGE UNIT

3 - PUMPING UNIT

4 - JET MIXER

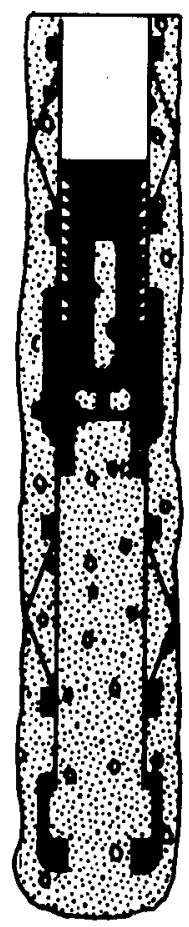

JOB COMPLETED
TOP PLUG SEATED

BOTTOM PLUG SEATED

VALVE CLOSED

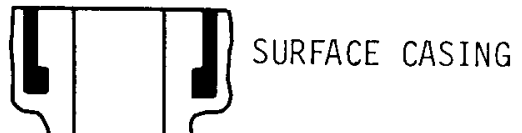

PRODUCTION

TOP PLUG

CENTRAL IZER

BOTTOM PLUG

SUPER SEAL

FLOAT COLLAR
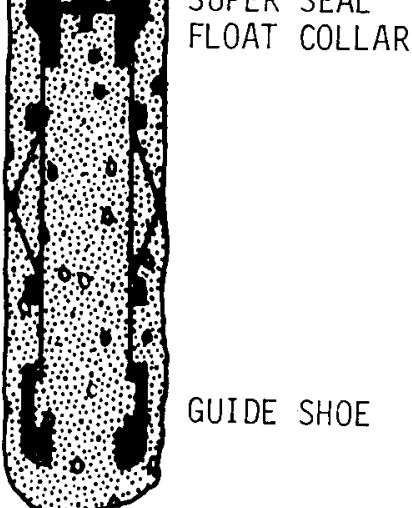

GUIDE SHOE

FIGURE 3-16. - Typical primary cementing job 

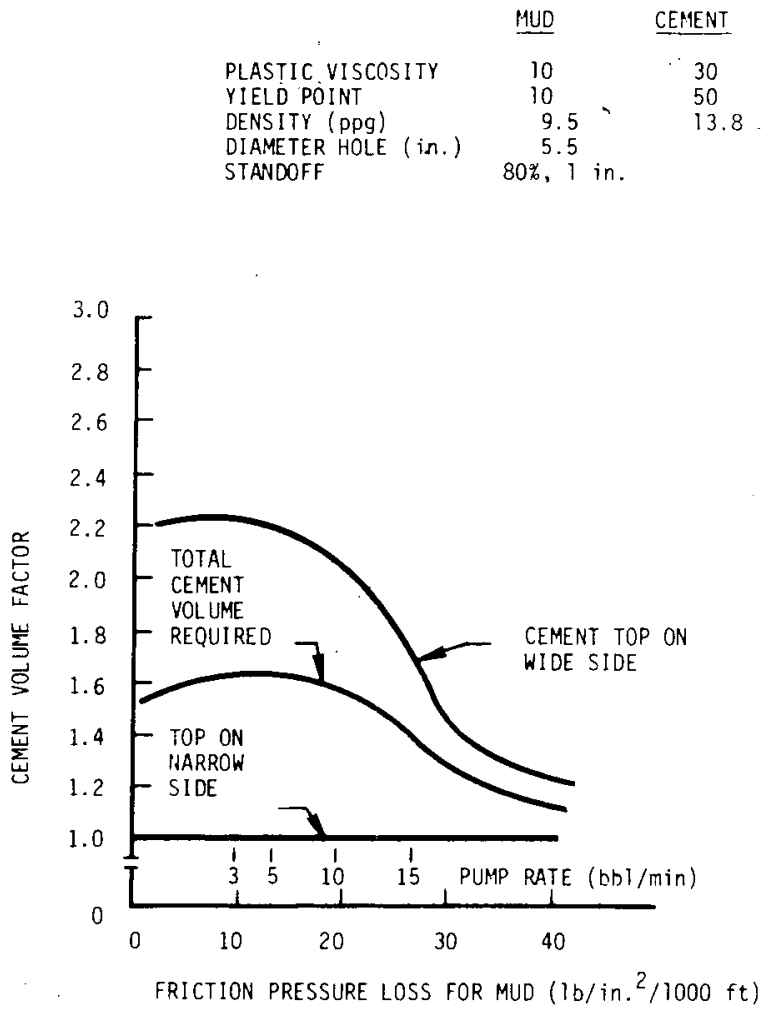

FIGURE 3-17. - Type of curve that can be designed for individual conditions to determine additional cement, to assure coverage of the narrow side of eccentric annulus. Muliply volume factor times volume of annulus from shoe to desired cement column height.

conditioning agent such as Flo-Chek ${ }^{\circledR}$. Another 5 to $10 \mathrm{bbl}$ of water are recommended to follow prior to pumping cement. The intent of this procedure is to assure that the mud cake be cleared of the casing wall and hole annulus such that mud and cement will not interdisperse.

Prior to pumping the full slug of cement, Shryock and Smith recommended that the top cementing plug be released so that 3 to $5 \mathrm{bbl}$ of cement slurry remain in the pump lines. The objective is to switch to mud without shutting down the pumps to maintain a steady pump rate throughout the displacement. Displacement continues until the top plug bottoms out on the bottom plug or the pump pressure reaches a predetermined maximum, indicating that the annulus is full. At this point, the check valve in the float collar should hold the hydrostatic head of the cement in the annulus. It is recommended that pressure be held on the cement for 18 to 24 hr until the cement hardens. 
The excess of cement which sits inside the casing can be drilled out with less effort than is required to repair a poor cementing job.

It had been thought previously that the bottom cement plug displaced mud from the casing wall such that mud and cement could not mix. In the next subsection we will examine the bottom plug in detail to determine if the plug has mechanical characteristics such that it will:

\section{a. Create a scraping action so as to remove mud film from the wall. \\ b. Survive the geothermal wall temperatures.}

\subsubsection{Cement wiper Plugs}

A variety of cement wiper plugs are available to lead and follow cement in a geothermal well. While most plugs are molded to shape around a drillable center material such as cast aluminum, the bottom plug also contains a frangible diaphragm which can be ruptured when the plug bottoms out on the float collar. Cement passes through the bottom plug and float collar until the top cement plug seats in the bottom plug and terminates the cementing. A general schematic of the top and bottom plug is shown in Figure 3-18 and a list of the various moldable rubbers is given

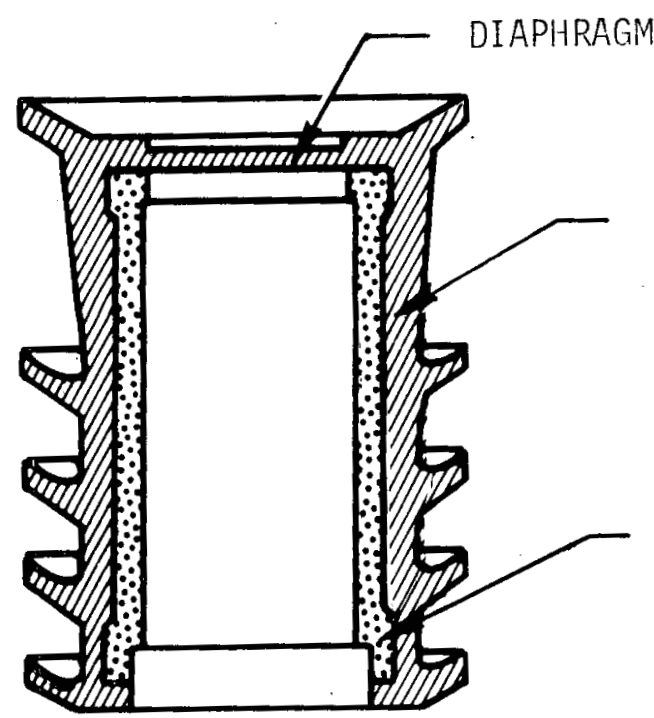

BOTTOM PLUG

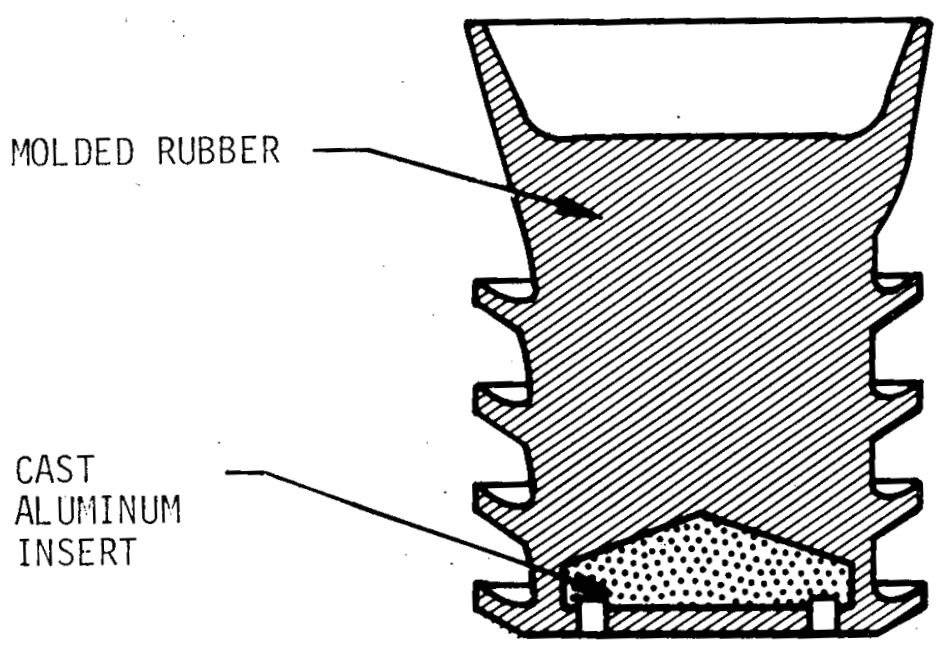

TOP PLUG

FIGURE 3-18. - Top and bottom wiper plugs. Diaphragm in bottom plug ruptures with pressure increase to allow slurry passage. Solid plug, right, follows slurry. All plug material is drillable. 
in Table 3-9. A 7-in. top cementing plug is shown in Figure 3-19. Please note that these are general maximum temperature limits based on static temperature material testing.

In a transient temperature environment it may be possible to use elastomers at temperatures up to $5000 \mathrm{~F}$ for $24 \mathrm{hr}$ or more. The following high temperature elastomers were developed by L'Garde, Inc. under DOE contract for use as geothermal packers.

$24 \mathrm{hr}$ temperature

Type capability

$\begin{array}{ll}267-I I-8 & \text { EPDM } \\ 266-I I-3 & \text { EPDM/VITON } \\ 291-I I-2 & \text { FIuorelastomer } \\ & \text { AFLAS }\end{array}$

$500^{\circ} \mathrm{F}$

$500^{\circ} \mathrm{F}$

AFLAS

$500^{\circ} \mathrm{F}$

TABLE 3-9. - Common materials for cementing plugs

\begin{tabular}{|c|c|c|c|}
\hline $\begin{array}{l}\text { Common } \\
\text { name }\end{array}$ & $\begin{array}{l}\text { Chemical } \\
\text { name }\end{array}$ & Major attribute & $\begin{array}{l}\text { Maximum } \\
\text { temperature } \\
\text { range }\left({ }^{\circ} \mathrm{F}\right)\end{array}$ \\
\hline $\begin{array}{l}\text { Synthetic } \\
\text { rubber }\end{array}$ & $\begin{array}{l}\text { Synthetic } \\
\text { polyisoprene }\end{array}$ & $\begin{array}{l}\text { Impact strength } \\
\text { tear resistance } \\
\text { cut growth }\end{array}$ & -50 to +300 \\
\hline Neoprene & Chloroprene & $\begin{array}{l}\text { Oil and gas resistance } \\
\text { abrasion resistance }\end{array}$ & -40 to 240 \\
\hline EDPM & $\begin{array}{l}\text { Ethylene } \\
\text { Propylene }\end{array}$ & General purpose & -60 to 300 \\
\hline Nitrile & $\begin{array}{l}\text { Butadiene } \\
\text { Acrylonitrile }\end{array}$ & $\begin{array}{l}\text { Oil resistance } \\
\text { abrasion resistance }\end{array}$ & -60 to +300 \\
\hline Butadiene & Polybutadiene & General purpose & -60 to +180 \\
\hline ButyI & $\begin{array}{l}\text { Isobutylene } \\
\text { isoprene }\end{array}$ & $\begin{array}{l}\text { Air retention } \\
\text { ozone resistance }\end{array}$ & -50 to +300 \\
\hline
\end{tabular}




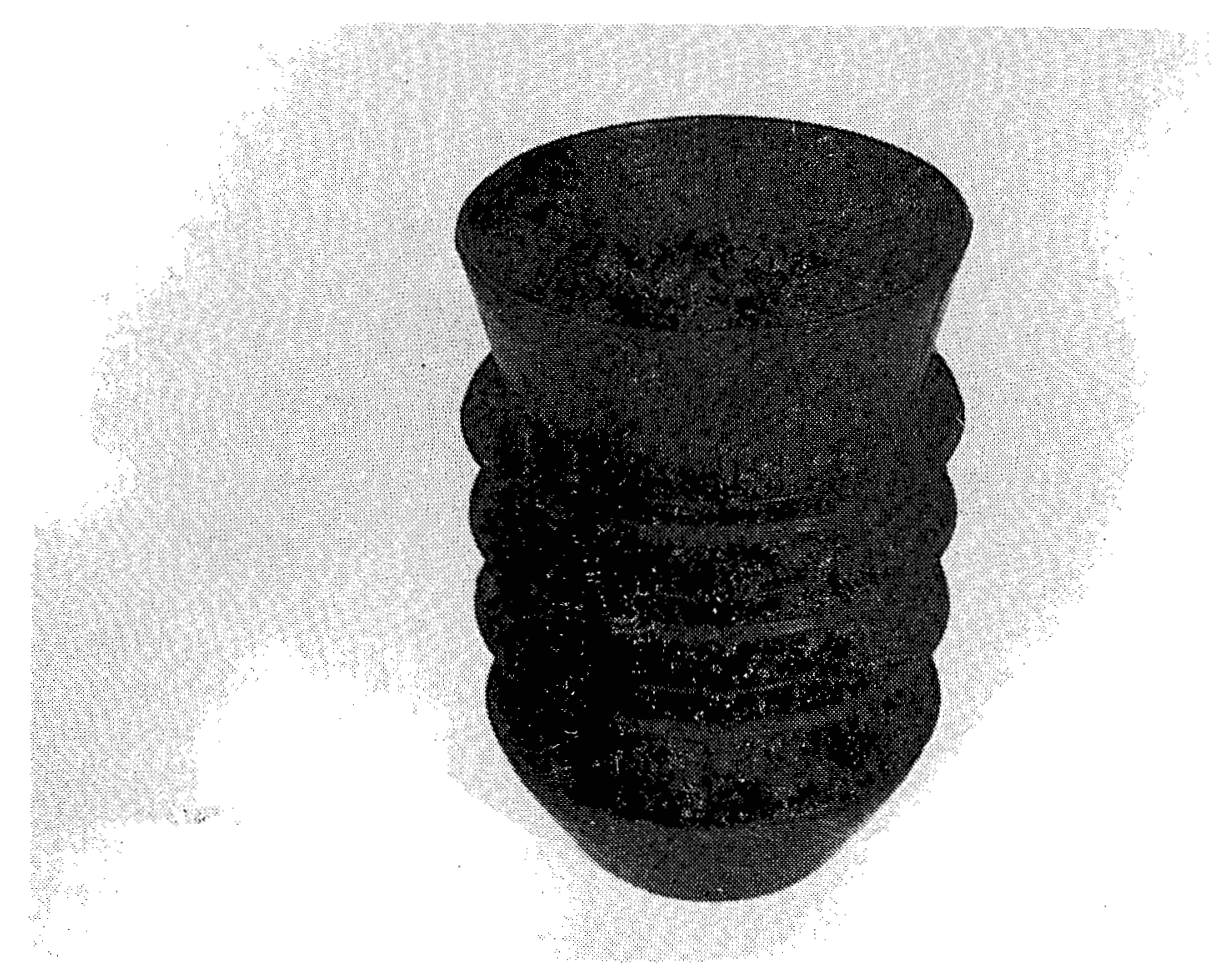

FIGURE 3-19. - 7-in. top cementing plug recommended for 17 to 38 lb/ft casing.

In general, one cement plug size is recommended by the manufacturer to cover a range of casing weights within a given casing size. For instance, a 7-in. cementing plug is recommended to cover the 7-in. casing from 17 to $38 \mathrm{lb} / \mathrm{ft}$ of casing weight. In Figure 3-20, casing internal diameter has been plotted versus casing weight for a range of casings commonly used in geothermal cementing. In addition, the manufacturer's recommended cement plug size is indicated for a range of casing weights.

In Figure 3-2l, the degree of interference fit between the various casing weights and recommended cement plug sizes is shown as a function of casing internal diameter. Interference fit is simply defined as cement plug outside diameter minus casing internal diameter. In Table 3-10, the degree of interference fit has been 1 isted versus casing weight for a 7-in. casing. At the low end of casing weight $(17 \mathrm{lb} / \mathrm{ft})$, the plug outside diameter exceeds the casing inside diameter by $0.087 \mathrm{in.} \mathrm{At} \mathrm{the} \mathrm{high}$ end of the casing weight (38 lb/ft), the plug outside diameter minus casing inside diameter is $0.705 \mathrm{in.}$ 
CASING OD (in.)

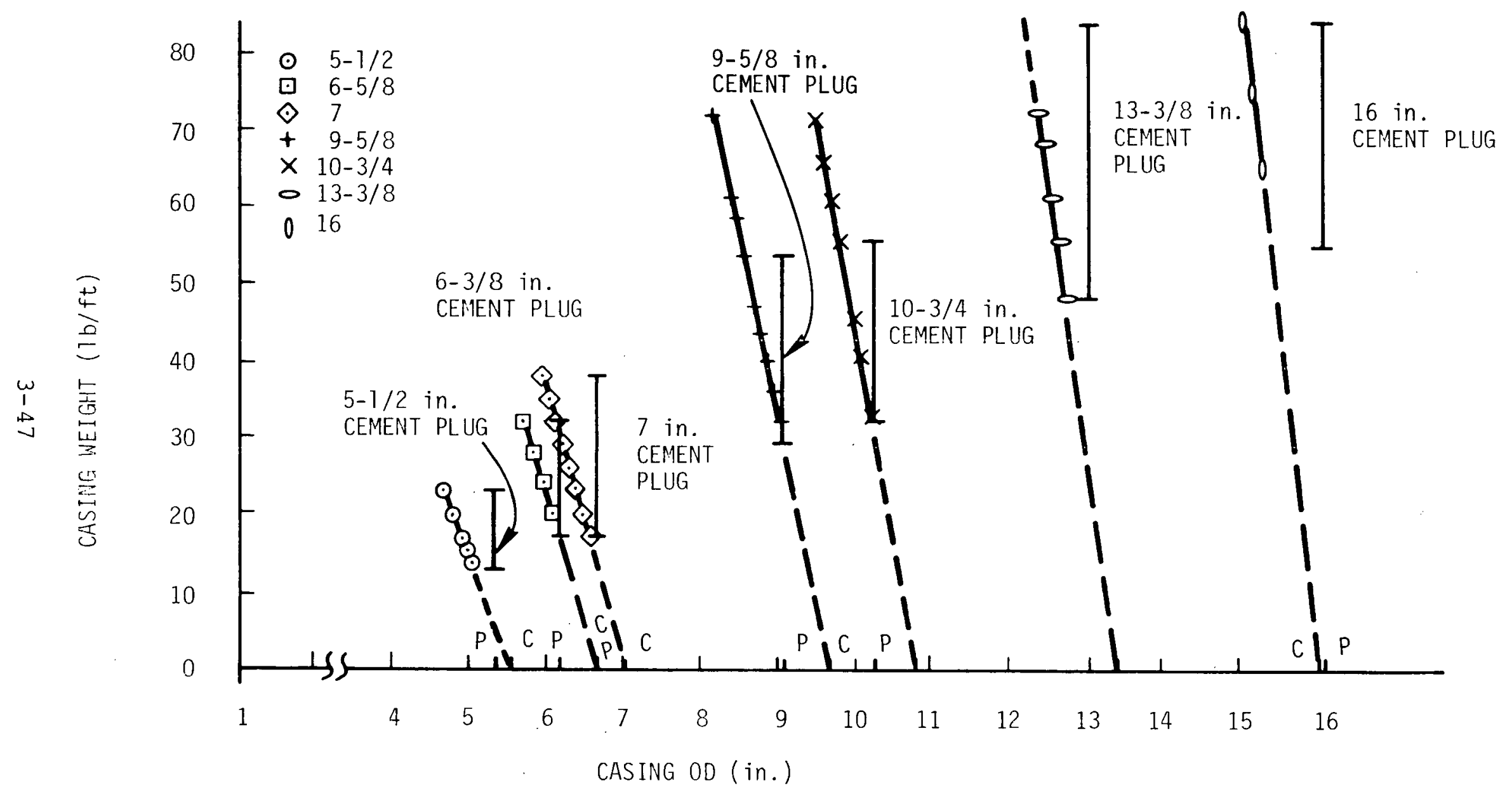

FIGURE 3-20. - Casing internal diameter and cement plug external diameter versus casing size and weight. 


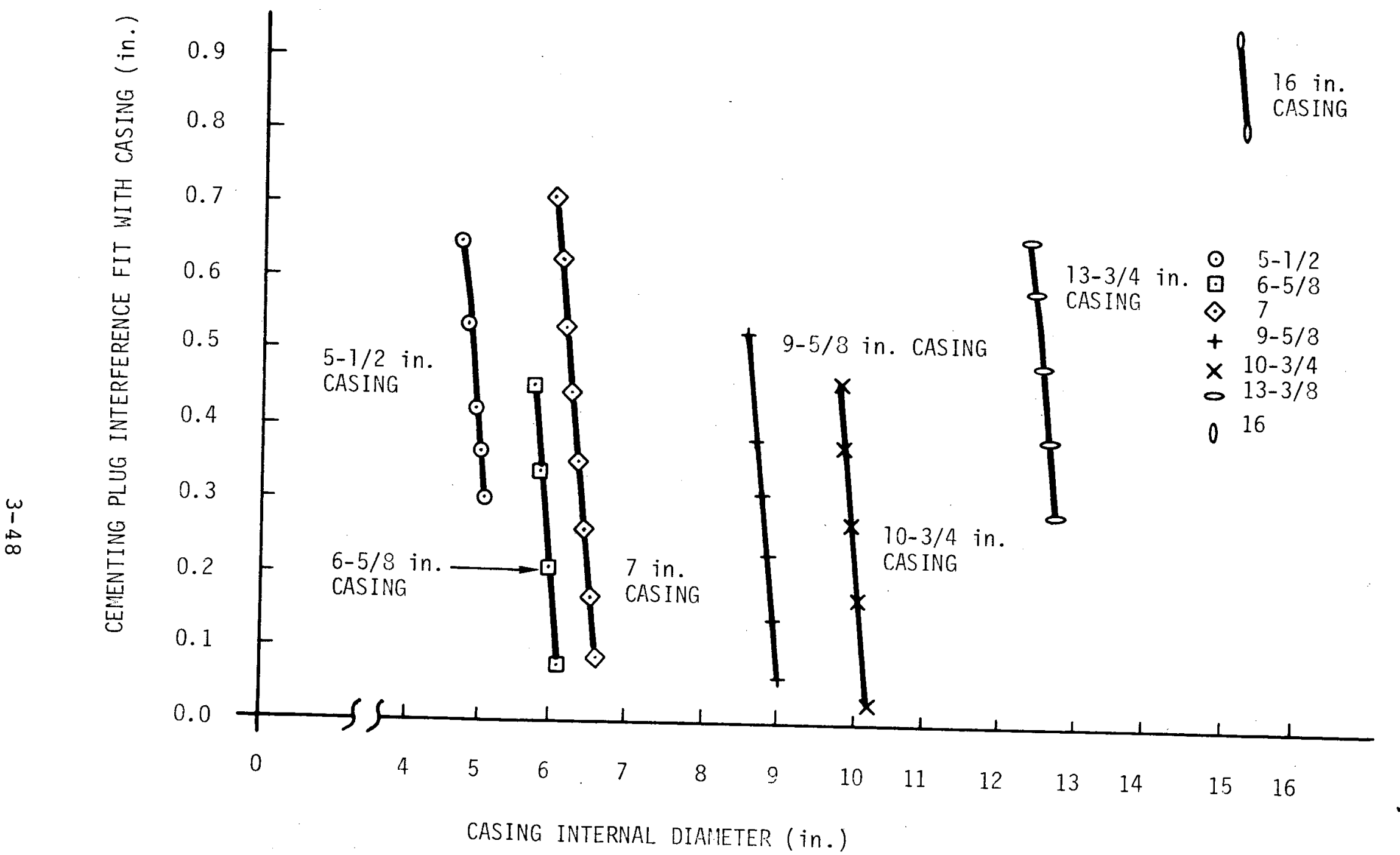

FIGURE 3-21. - Cement plug interference fit with casing internal diameter for various casing sizes and manufacturer recommended cementing plugs. 

TABLE 3-10. - Interference between top cementing plug

\begin{tabular}{|c|c|c|c|}
\hline $\begin{array}{c}\text { Casing Weight } \\
\text { lb/ft }\end{array}$ & $\begin{array}{l}\text { Casing Inside } \\
\text { Diameter (in.) }\end{array}$ & $\begin{array}{c}\text { Cementing Plug } \\
\text { Outside Diameter } \\
\text { (in.). }\end{array}$ & $\begin{array}{l}\text { Interference } \\
\text { (in.) }\end{array}$ \\
\hline 17 & 6.538 & 6.625 & 0.087 \\
\hline 20 & 6.456 & & 0.169 \\
\hline 23 & 6.366 & & 0.259 \\
\hline 26 & 6.276 & & 0.349 \\
\hline 29 & 6.184 & . & 0.441 \\
\hline 32 & 6.094 & & 0.531 \\
\hline 35 & 6.004 & & 0.621 \\
\hline 38 & 5.920 & & 0.705 \\
\hline
\end{tabular}


While 7-in casing is rarely cemented in place during a well completion, the problem which we are about to describe is generic and will apply equally well to larger casings and cementing plugs.

\subsubsection{Cement Plug Pressure Loading on Casing Side Walls}

It was thought at first that the amount of interference between the cementing plug and the casing would result in highpressure loads between the plug and the wall. In order to measure the applied pressure, we purchased a plastic tube with an internal diameter of 6.396 in. and a wall thickness of approximately $1 / 8$ in. This tube represents the approximate internal diameter of a 7-in., $23 \mathrm{lb} / \mathrm{ft}$ casing (6.366 in.).

Upon inserting the cementing plug into the plastic tube, we observed that the three flat central discs bent back such that they were also conical in shape. A schematic representation of the undisturbed and interference fit cementing plug is shown in Figure 3-22.

A brief experiment was conducted to determine the modulus of elasticity of the plastic tube tangentialiy and longitudinally. In order to determine the modulus, two aluminum plates were machined with o-ring grooves such that the ends of the tube could be sealed and pressurized. The tube was pressurized (Figure 3-23), and the strain on the tube was measured as a function of internal air pressure. Since the hoop stress on a cylinder under pressure is

$$
\sigma=\frac{\operatorname{Pr}}{t}
$$

where

$$
\begin{aligned}
\sigma & =\text { stress } \\
\mathrm{P} & =\text { internal pressure } \\
\mathbf{r} & =\text { radius } \\
\mathrm{t} & =\text { wall thickness }
\end{aligned}
$$

and the stress and strain are related by the relationship

$$
E=\sigma / \varepsilon
$$



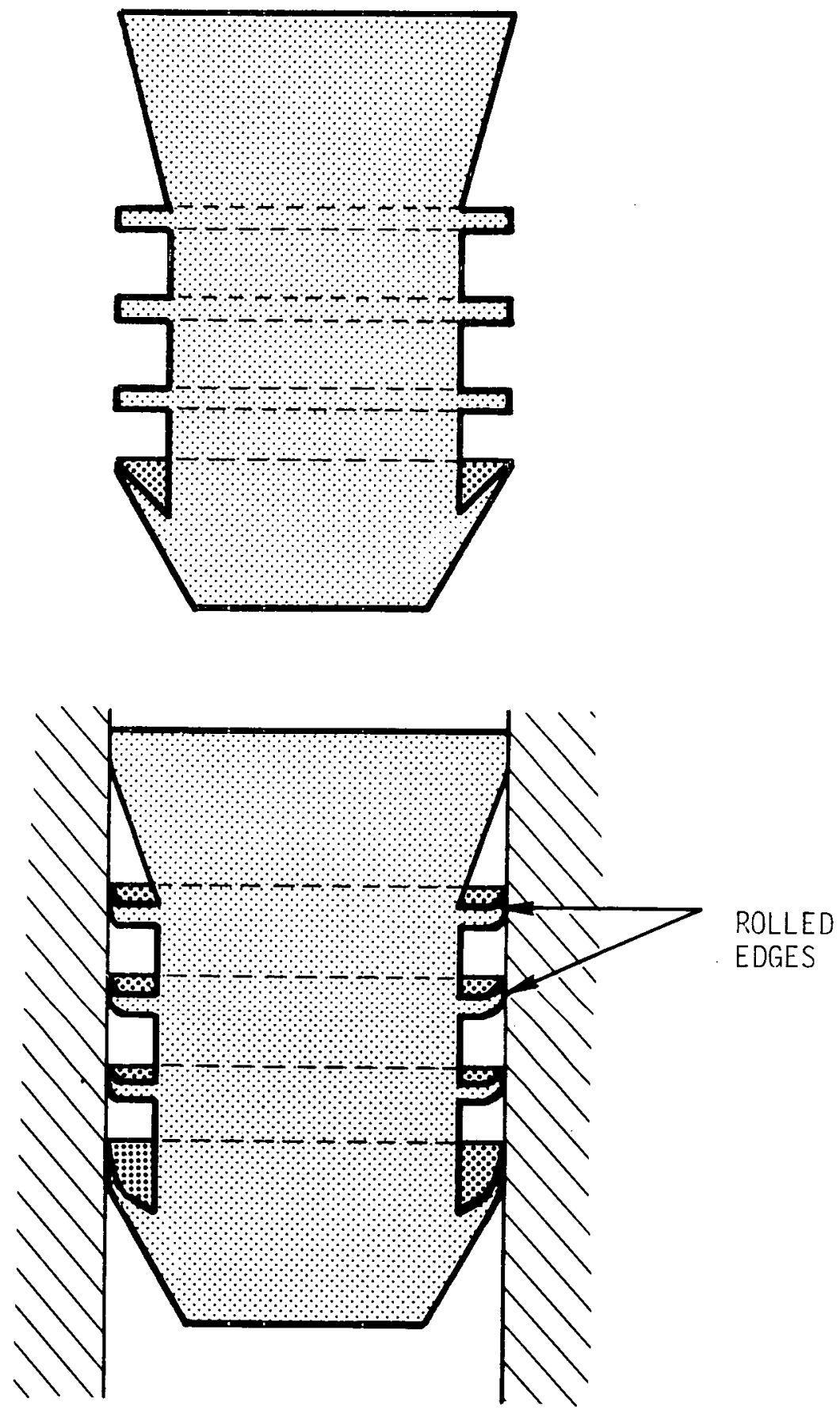

FIGURE 3-22. - Schematic representation of cementing plug interference fit into casing. 


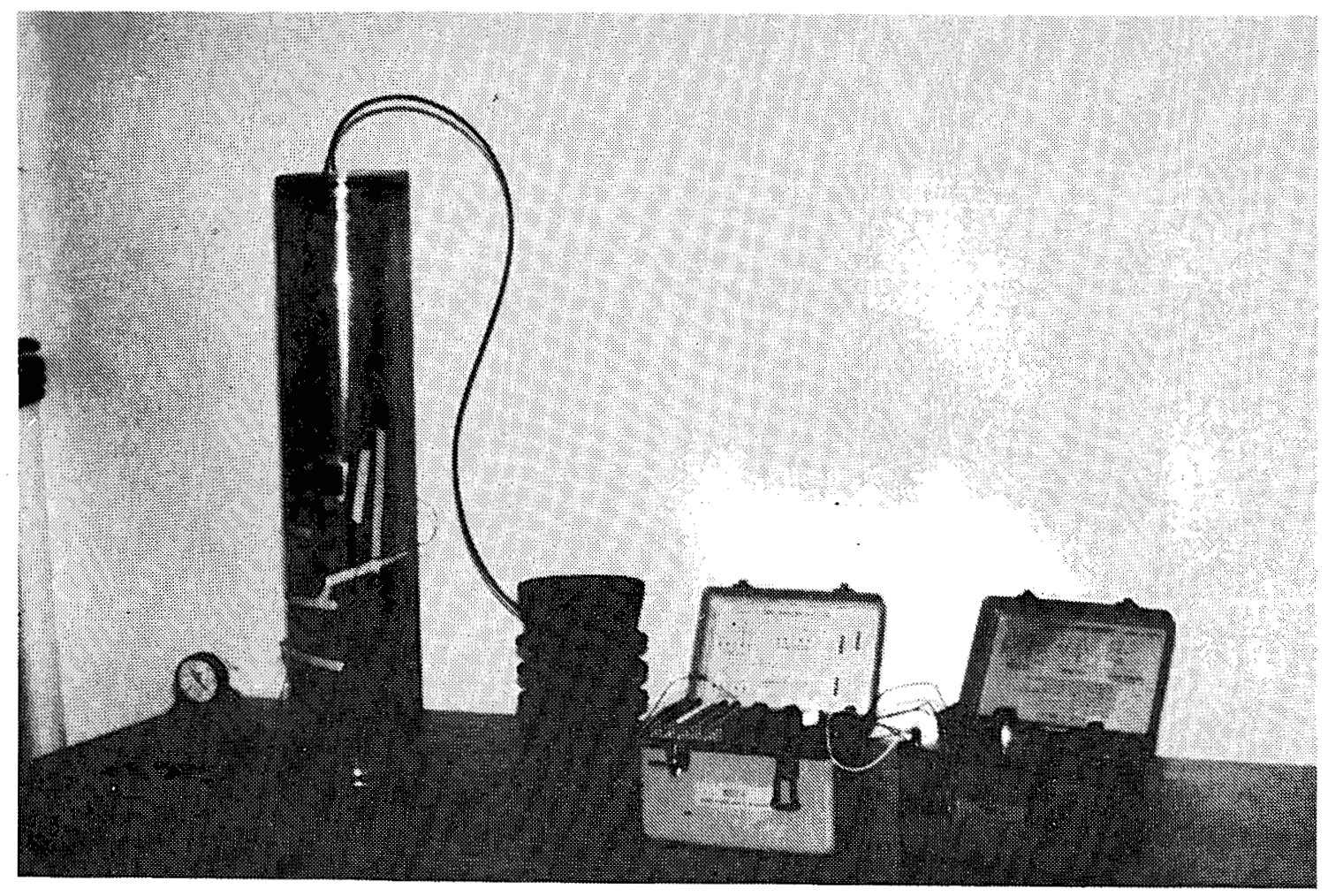

FIGURE 3-23. - Plastic tube-strain-gaged and pressurized to measure internal pressure versus strain. 
where

$$
\begin{aligned}
& \mathrm{E}=\text { Modulus of elasticity } \\
& \varepsilon=\text { strain (in./in.) }
\end{aligned}
$$

By calibrating the plastic tube it was possible to determine the internal pressure generated by the cementing plug as a function of measured hoop strain. This calibration is shown in Figure 3-24, and the corresponding data point for the stress due to the cementing plug is noted.

The cementing plug was pushed by hand into the plastic tube and the stress level was recorded. The tangential strain was measured to be $82 \mathrm{\mu in./in.,} \mathrm{corresponding} \mathrm{to} \mathrm{an} \mathrm{internal} \mathrm{pressure}$ of $3.65 \mathrm{lb} / \mathrm{in}^{2}$ generated by the cementing plug on the wall of the tube. We were surprised that the applied wall pressure generated by the plug was so low.

This pressure is exerted by each ring over a longitudinal length of approximately $1 / 2$ in. for the entire inside circumference of the tube. Therefore, the force generated by each ring was approximately

$$
\begin{aligned}
\mathrm{F}_{\text {wall }} & =\mathrm{PA}_{\mathrm{w}} \\
& =\left(3.65 \mathrm{lb} / \mathrm{in}^{2}\right)(\pi)(6.396 \times 0.5) \\
& =36.6 \mathrm{lb}
\end{aligned}
$$

\subsubsection{Thermal Analysis of Wiper Ring}

The cementing plug of interest is molded from acrylonitrile butadiene, commonly referred to as Nitrile or Buna-N. This material is widely used by the seal industry as o-ring material. The chief attraction is the ability of the material to resist petroleum products and the wide service range of temperature $\left(-60^{\circ}\right.$ to $\left.+300^{\circ} \mathrm{F}\right)$. The performance of nitrile at $300^{\circ} \mathrm{F}$ is poor; normal maximum temperature is $+250^{\circ} \mathrm{F}$, which is below the temperatures normally encountered in a geothermal well. Due to the low thermal diffusivity of the rubber and the good thermal contact between the rubber and the wall of the casing, the surface temperature of the rubber will follow the wall temperature very closely, as shown in the analysis which follows. 


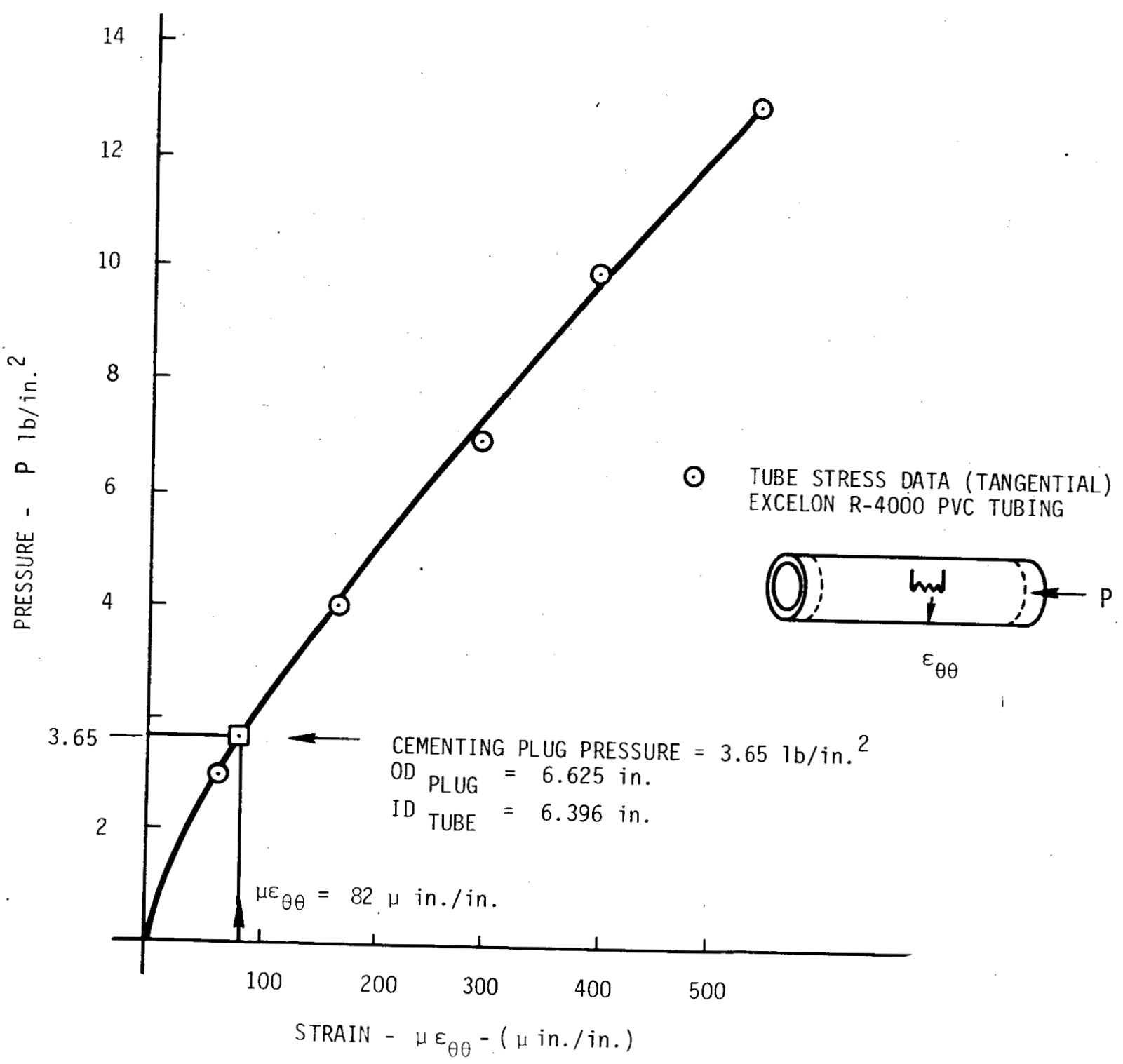

FIGURE 3-24. - Internal pressure versus strain 7-in. cementing plug. 
If we consider a rubber slab to be a semi-infinite solid, we can calculate the thermal response of the slab subjected to an elevated temperature. We use the following properties of the nitrile rubber:

$$
\begin{aligned}
& \mathrm{k}=0.1 \mathrm{Btu} / \mathrm{hr} \mathrm{ft}^{\circ} \mathrm{F} \\
& \rho=701 \mathrm{~b}_{\mathrm{m}} / \mathrm{ft}^{3} \\
& c_{p}=0.5 \mathrm{Btu} / 1 \mathrm{~b}_{\mathrm{m}} \mathrm{O}_{\mathrm{F}} \\
& \alpha=\frac{\mathrm{k}}{\rho \mathrm{C}_{\mathrm{p}}}=0.0029 \mathrm{ft}^{2} / \mathrm{hr}
\end{aligned}
$$

The interface conductance between the rubber and the wall will be approximately $h=100 \mathrm{Btu} / \mathrm{hr} \mathrm{ft}^{2} \mathrm{OF}$. Solving for the transient time history of the rubber slab, we get the results shown in Table 3-11.

TABLE 3-11. - Transient time history of rubber slab

\begin{tabular}{|c|c|}
\hline $\begin{array}{c}\text { Time } \\
(\sec )\end{array}$ & $\frac{T-T_{i}}{T_{\infty}-T_{i}}$ \\
\hline 1 & 0.6 \\
\hline 10 & 0.9 \\
\hline 100 & 0.94 \\
\hline
\end{tabular}

At typical cement pumping rates, the wall temperature of the cement plug will come to within 94 percent of the final wall temperature in approximately $300 \mathrm{ft}$. Since the static wall temperature increases from the surface to the maximum geothermal value, the plug wall temperature will also rise to the operating temperature as the cement is pumped downhole. (See Figure 3-25 for a typical static wall temperature profile in the Imperial valley.) 
TE:IPERATURE $\left({ }^{\circ} \mathrm{C}\right)$

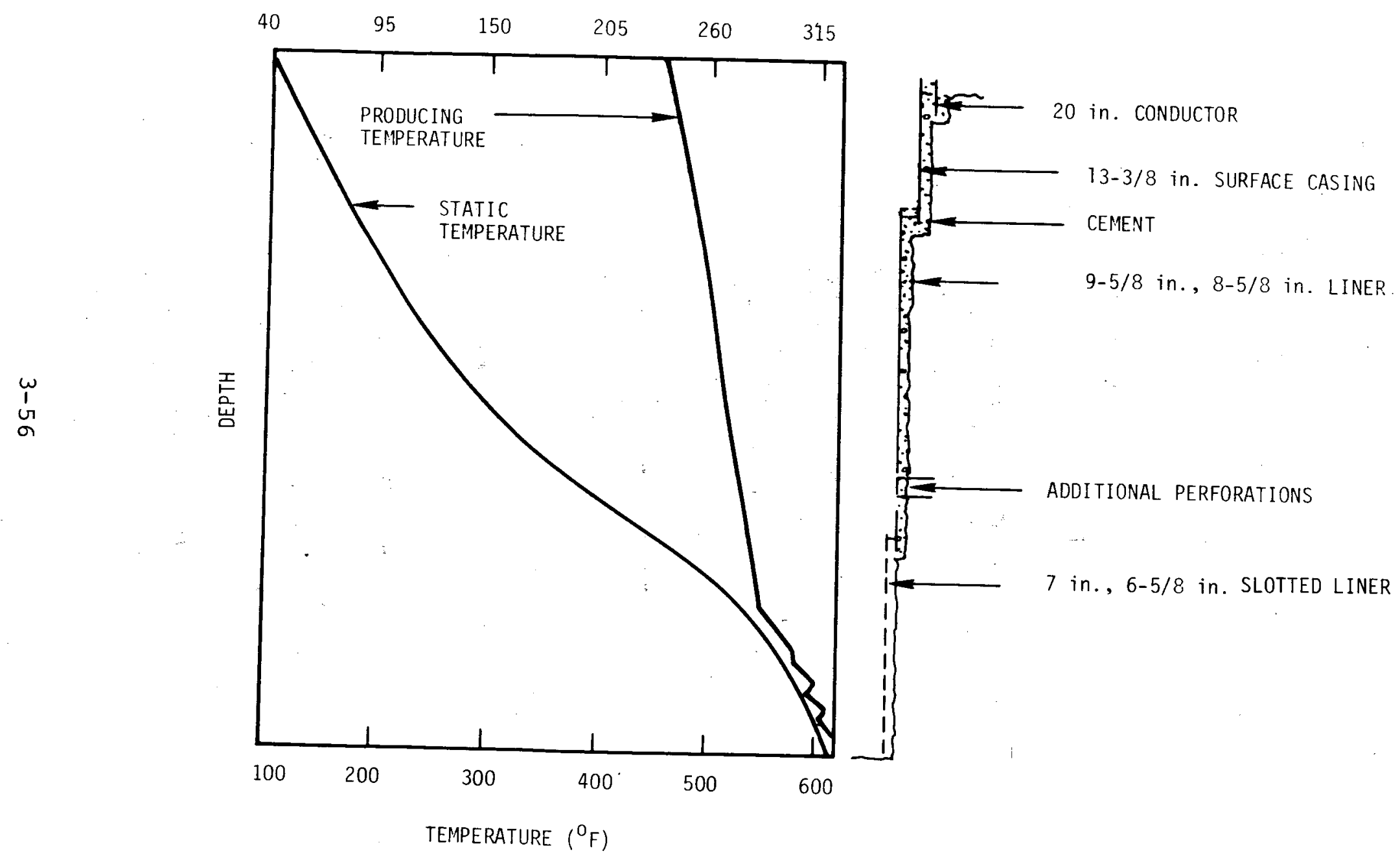

FIGURE 3-25. - Temperature 
In conclusion, the plug wall temperature will quickly rise above the service limit for the plug as the cement is pumped downhole.

\subsubsection{Cementing Plug Wear}

For elastomers an abradability index has been defined ( $\underline{76}$ ) as :

$$
\begin{aligned}
\gamma & =\frac{\text { Abraded Volume }}{\text { Frictional Work }} \\
& =\frac{\Delta V}{F_{N}^{\mu L}}
\end{aligned}
$$

where

$$
\begin{aligned}
\Delta \mathrm{V} & =\text { Abraded volume } \\
\mathrm{F}_{\mathrm{N}} & =\text { Normal load } \\
\mu & =\text { Coefficient of friction } \\
\mathrm{L} & =\text { Distance traveled. }
\end{aligned}
$$

In order to determine the frictional work, it is necessary to determine the friction coefficient as a function of temperature and sliding velocity. This variation has been determined by Grosch (77), Figure 3-26, in the form of a master curve of fricton coefficient versus sliding velocity. The master curve is displaced to the right as temperature increases with data taken at a maximum temperature of $85^{\circ} \mathrm{C}\left(185^{\circ} \mathrm{F}\right)$.

Next, we determine the variation of abradability, $\gamma$, with temperature. Again, this variation has been determined by Grosch and Schallamach (78), Figure 3-27, over a temperature range to $100^{\circ} \mathrm{C}\left(212^{\circ} \mathrm{F}\right)$. We see from Figure 3-27 that abradability increases with increasing temperature.

Utilizing both the friction coefficient versus temperature and velocity, Figure 3-26, and the abrasion coefficient versus temperature, Figure 3-27, we can calculate the amount of cement plug material removed up to the limit of the available data (approximately $200^{\circ} \mathrm{F}$ ). 
FRICTION AND WEAR

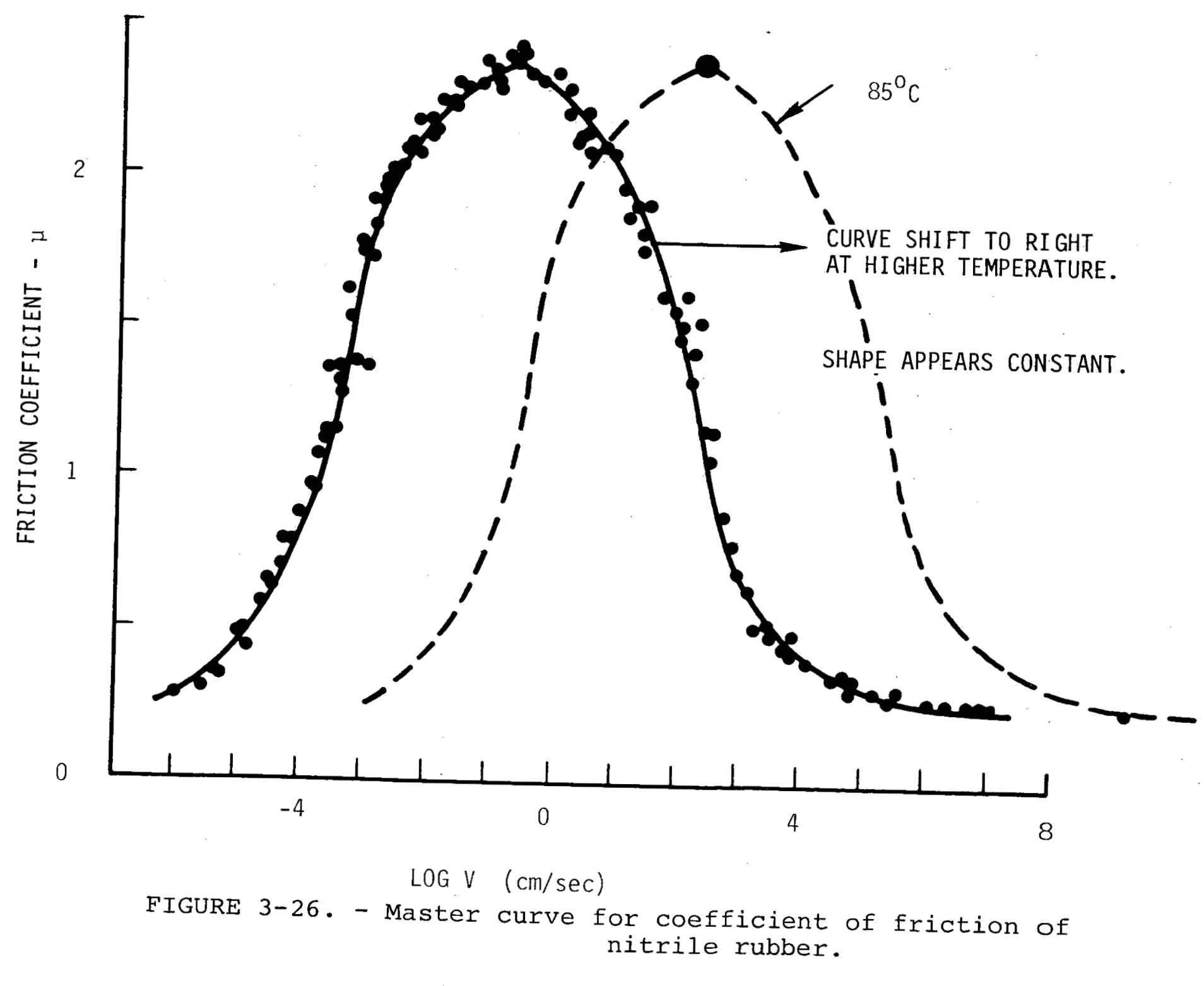




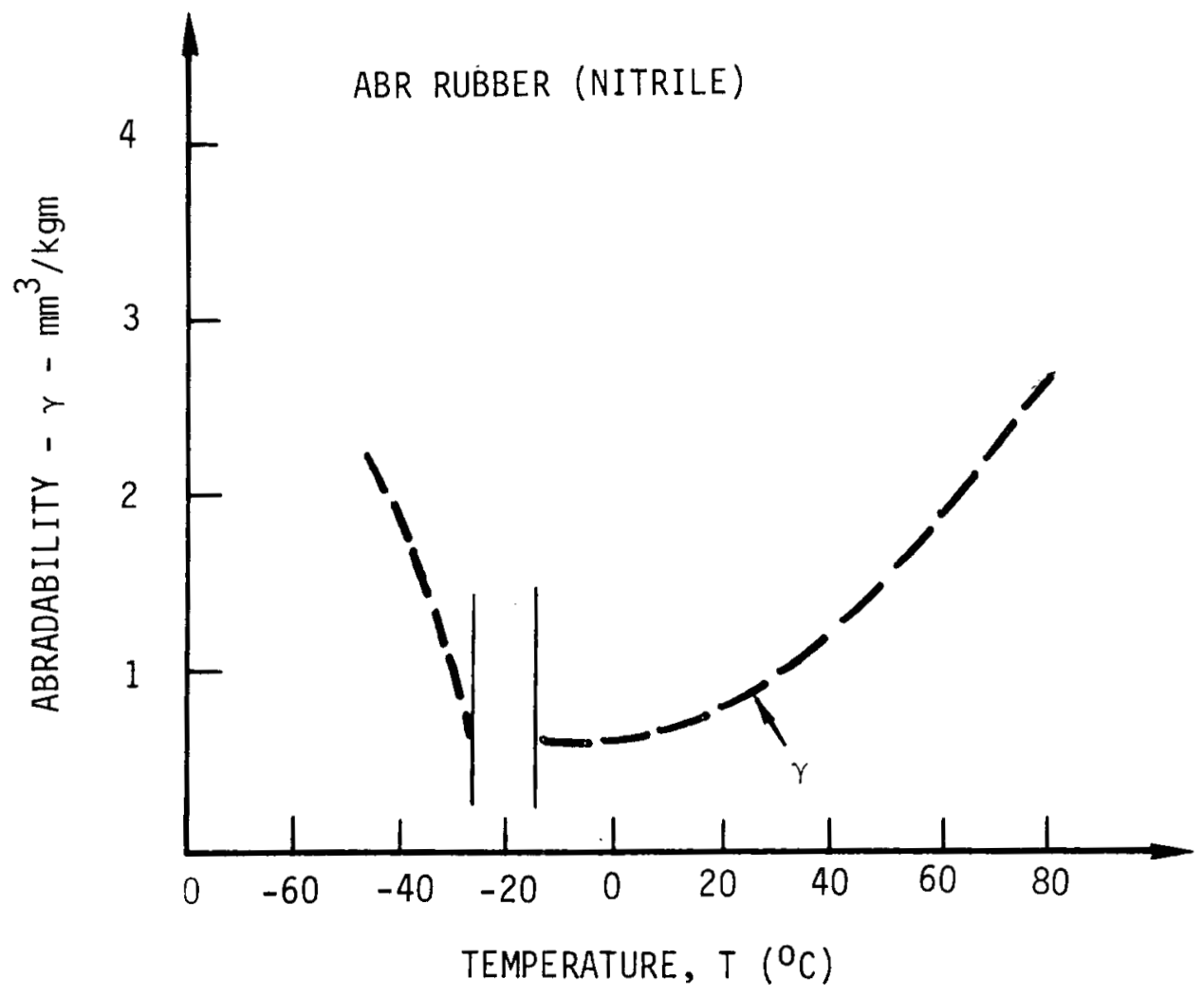

FIGURE 3-27. - Abradability - $\gamma-\mathrm{mm}^{3} / \mathrm{kg} \cdot \mathrm{m}$.

Using the 7-in. cementing plug shown in Figure 3-19, we can calculate the amount of material removed at the maximum temperature limit of the data $\left(85^{\circ} \mathrm{C}\right)$. Assume the following conditions:

a. Temperature $-85^{\circ} \mathrm{C}$

b. Cementing plug pressure $-3.65 \mathrm{lb} / \mathrm{in} .^{2}$

c. Outside diameter plug - 6.62 in.

d. Inside diameter casing -6.37 in.

e. Distance traveled - $1000 \mathrm{ft}$

f. Contact length $-1 / 2$ in. longitudinal

g. Cementing plug wall velocity $-90 \mathrm{~cm} / \mathrm{sec}$

From Figures 3-26, 3-27, and 3-28, we get the following values.

$$
\mu=2.4
$$

$$
\gamma=2.6 \frac{\mathrm{mm}^{3}}{\mathrm{~kg} \cdot \mathrm{m}}
$$




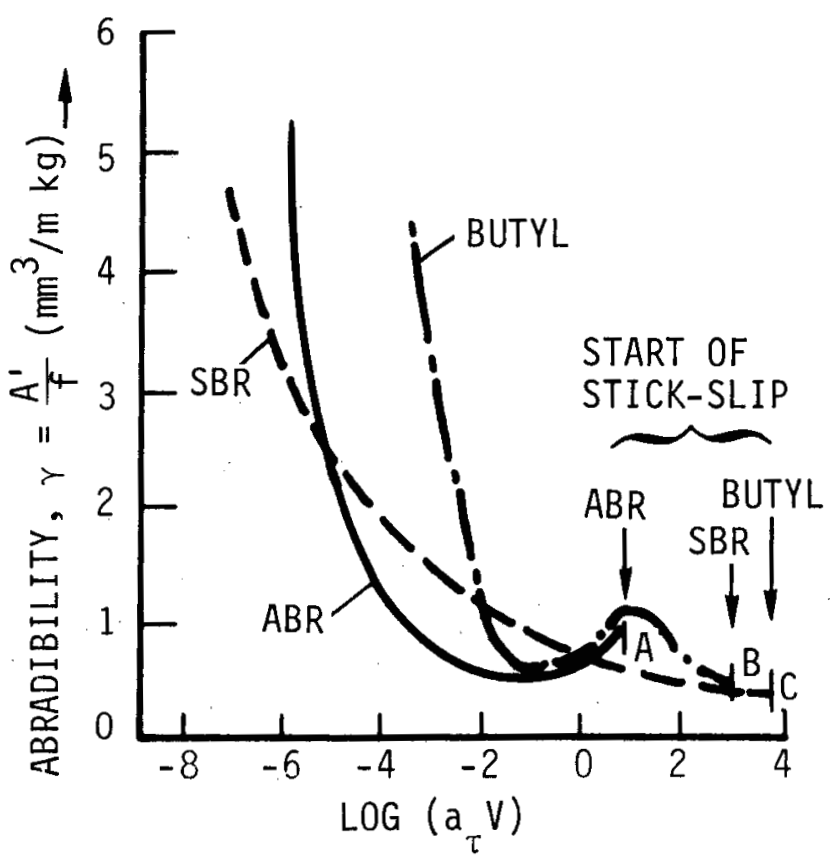

FIGURE 3-28. - Master curves obtained experimentally for the abrasion of three unfilled rubbers

The frictional work is thus

$$
\begin{aligned}
\text { Work } & =E_{N^{\mu L}} \\
& =(36.61 \mathrm{~b})(2.4) \quad(1000) \\
& =87840 \mathrm{ft} / 1 \mathrm{bm}
\end{aligned}
$$

Therefore, the volume lost is

$$
\begin{aligned}
\Delta V & =\gamma \text { (work) } \\
& =(2.6)(87840) \quad(0.454 \mathrm{~kg} / 1 \mathrm{~b})\left(\frac{\text { meter }}{3.28 \mathrm{ft}}\right)
\end{aligned}
$$

$$
\Delta \mathrm{V}=31612 \mathrm{~mm}^{3}
$$


For the plug diameter of $6.62 \mathrm{in.}(168.3 \mathrm{~mm})$ and a contact width of $1 / 2 \mathrm{in.}(12.7 \mathrm{~mm})$, the resulting wear length is

$$
\begin{aligned}
\Delta l & =\frac{\Delta V}{(\pi D)(t)}=\frac{31612}{(\pi)(168.3)(12.7)} \\
\Delta l & =4.71 \mathrm{~mm} \\
& =0.185 \mathrm{in} .
\end{aligned}
$$

Since this is the wear length on the thickness of the outer edge, the change in diameter is

$$
\Delta \mathrm{D}_{\text {wear }}=2(\Delta \ell)=0.370 \mathrm{in} \text {. }
$$

Thus the new plug diameter is

$$
\begin{aligned}
\begin{array}{l}
D_{\text {worn }} \\
\text { plug }
\end{array} & O D_{\text {plug }}-\Delta D_{\text {wear }} \\
& =6.62-0.370 \\
D_{\text {worn }} & =6.25 \mathrm{in} . \\
\text { plug } &
\end{aligned}
$$

From this brief analysis, it is evident that the interference fit between the rubber plug and the casing will wear away in less than $1000 \mathrm{ft}$ of travel down the casing. Therefore, the wiper plug does not wipe cement from the wall, but acts only as a separator between the cement and what precedes or follows the lead and trailing plug, respectively.

\subsubsection{Cement Plug Effectiveness}

Although the intent of top and bottom cementing plugs is to separate cement from the drilling mud environment, it is evident, based on the analysis in the previous sections that the hostile geothermal environment will prevent the cementing plug from accomplishing its objective. Because elastomeric materials are limited to temperatures below $400^{\circ} \mathrm{F}$, low cost moldable rubber from which most cement plugs are fabricated will not survive the geothermal temperatures. The portion of the plug which wipes against the 
casing or open hole will be rapidly heated to wall temperatures, due to the poor thermal conductivity of the rubber and the good heat transfer between the plug and the hot wall of the geothermal well. At elevated temperatures the cement plug material which is in contact with the wall will be rapidly abraded away.

\subsubsection{Conclusions}

Cement plugs which are fabricated from low-cost moldable elastomeric materials will not survive the geothermal environments. The elements of the cement plug which contact the hot casing or wall of the open hole will be quickly heated to wall temperature and abraded away. At best the cement plug will act as a separator between the cement and what leads or follows it.

\subsubsection{Recommendations}

The authors recommend that cement plugs be specifically designed for the geothermal environment such that mud can be effectively removed from the casing wall. This design will probably include a disposable wiper ring such as a ceramic coated steel ring. The wiper ring would need to be designed such that it could survive the joints between pipes yet reseal once past the pipe joint.

\section{3 Technical Evaluation of Slotted Liner Washing}

\subsubsection{Introduction}

Limitations of liner washing methods have been described in subsection 2.4. In general, surface water is pumped out through the slots of the slotted liner and recirculated back to the surface. The purpose of slotted liner washing is primarily to remove drilling mud from the wall of the open hole and to clear the slots of any debris so that the geothermal fluids will flow through the porous medium which surrounds the open hole. The purpose of this subsection is to evaluate technically the effectiveness of slotted liner washing as a means of mud removal.

In a typical slotted liner application, a 7-in. outside diameter slotted liner is suspended in an 8-3/4-in. borehole with 1000 net feet of permeable sand providing the geothermal flow. The slot spacing is typically 16 slots per foot in a geometry similar to that shown in Figure 3-29. Slot geometry is typically 2 in. high by 0.25 in. wide. Spacing between the slot and the wall is on the order of 0.875 in. or 3.5 slot diameters. 


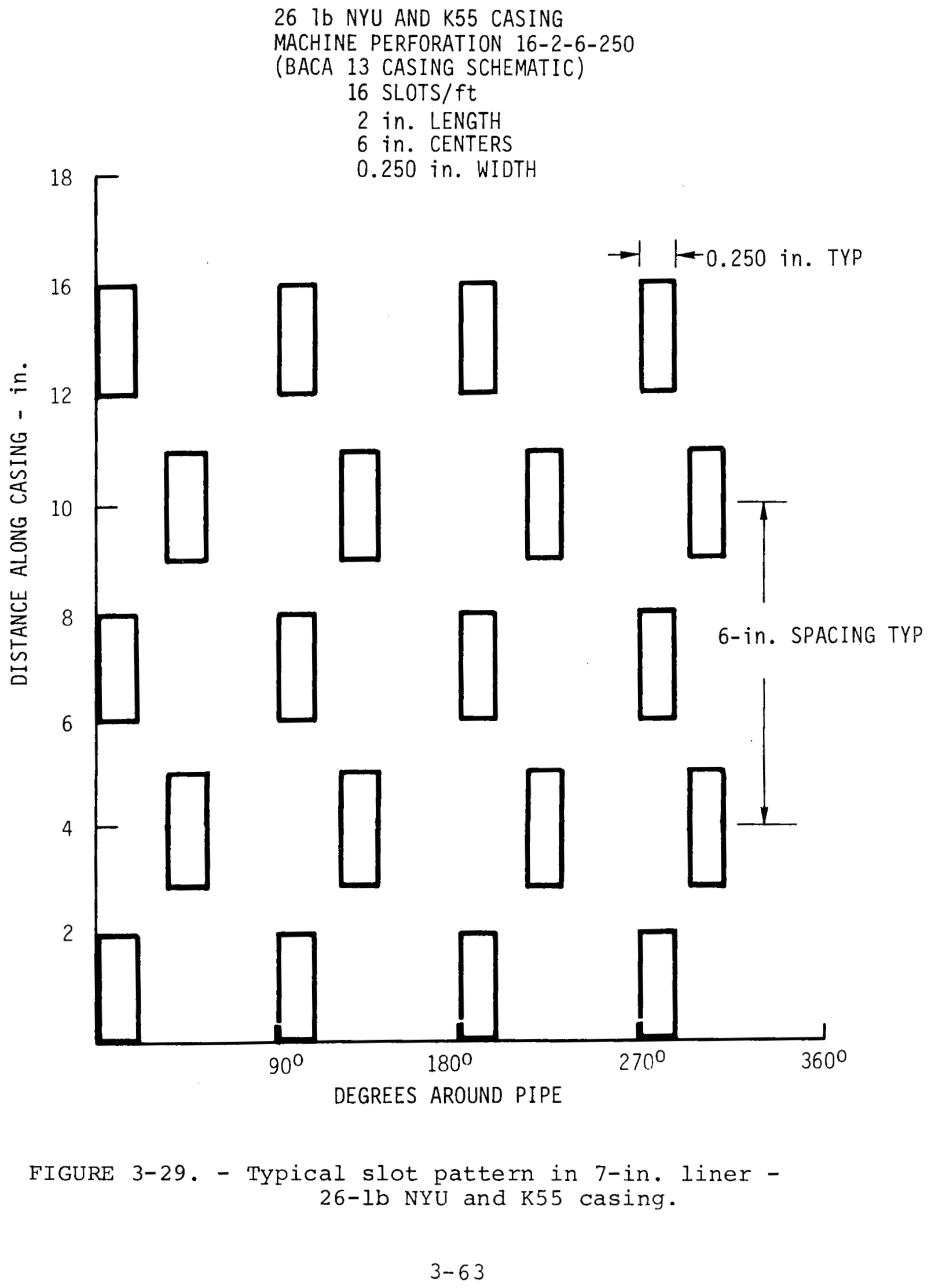


Surface pumps have a pumping capacity of approximately $15 \mathrm{bbl} / \mathrm{min}$ or $630 \mathrm{gal} / \mathrm{min}$. Washing tools described in subsection 2.4 may be set over a range between 1 ft to 5 ft of spacing. At 16 slots per foot and a slot area of 0.5 in. 2 , the maximum pumping rate will be spread over a slot area between 8 and 40 in. ${ }^{2}$.

The pumping rate of $630 \mathrm{gal} / \mathrm{min}$ corresponds to $1.417 \mathrm{ft} 3 / \mathrm{sec}$ flow rate through slots whose total area varies from $0.0555 \mathrm{ft}^{2}$ ( 8 in. ${ }^{2}$ ) to $0.2777 \mathrm{ft}^{2}$ (40 in.2). Therefore, the range of slot velocities at maximum pumping rate is 5.10 to $25.5 \mathrm{ft} / \mathrm{sec}$.

Because slotted liner washing must be accomplished over an interval of $1000 \mathrm{ft}$ or more, it is more likely that washing will be done in 3- to 5-ft intervals at surface pump rates corresponding to $5 \mathrm{bbl} / \mathrm{min}$. At the slot spacing given previously, this corresponds to a jet velocity more like

$$
v_{j e t}=1.70 \mathrm{ft} / \mathrm{sec}
$$

at

16 slots per foot

0.5 in. 2 per slot

3-ft washing length

$5 \mathrm{bbl} / \mathrm{min}$ surface pumping

The technical question then is as follows: "Can a jet of water at a slot velocity of 1.70 to $5.1 \mathrm{ft} / \mathrm{sec}$ wash the annular region between a 7-in. Iiner and an 8-3/4-in. borehole?" This question will be addressed in the following subsections.

\subsubsection{Description of Slotted Liners}

Perforated or slotted pipes are manufactured by saw-cutting longitudinal slots in the outer diameter of the pipes of interest. As saw-cut holes, they are generally thin (0.25 in. wide) and long ( 2 in.). General pipe perforating equipment can generate a slot over the following range of parameters (79):
a. Slot length
- up to $3-1 / 2$ in.
b. Slot width
-0.010 to $0.500 \mathrm{in}$.
c. Slot center-to-center distance - 6 in. typical. 
As the generated slots are saw-cut, there is not much flexibility in the slot configuration other than spacing and number per foot. The actual slots are nominally rectangular and spaced uniformly around the pipe circumference at intervals determined by the slot-generating apparatus (nominally 6-in. center-to-center between slots).

Surveying the Baca casing data reveals that many different combinations of slotted liners are used for completions. While early slots were generated by torch cutting, later slots were machine-generated. The majority of slots was machined at a density of 16 slots per foot, 2 in. long, on 6-in. centers, 0.250 in. wide.

\subsubsection{Fluid Dynamics of Flow Between a slotted Liner and an Open Hole}

The purpose of this subsection is to review the flow of a jet of water which emanates from a slot and impinges on a wall approximately 3.5 slot diameters downstream of the jet. Of particular interest is the flow field between adjacent jets where fluid dynamic shear would be the primary mechanism for mud removal.

A series of slots, as shown in Figure 3-29, is used to collect geothermal fluid. In slotted liner washing, flow rates between 5 and $15 \mathrm{bbl} / \mathrm{min}$ are pumped out between 3 to $5 \mathrm{ft}$ of slotted liner with typical spacing of 16 slots per foot. The mean flow velocity out of these slots will be between $1.70 \mathrm{ft} / \mathrm{sec}$ for nominal slotted liner conditions up to $5.1 \mathrm{ft} / \mathrm{sec}$ at maximum pumping rates. Nominal conditions are shown in Table 3-12.

TABLE 3-12. - Nominal slotted liner conditions

\begin{tabular}{|l|l|l|}
\hline \multicolumn{1}{|c|}{ Parameter } & Case No. 1 & Case No. 2 \\
\hline Slot spacing & $16 / \mathrm{ft}$ & $16 / \mathrm{ft}$ \\
Slot area & $0.5 \mathrm{in.}{ }^{2}$ & $0.5 \mathrm{in.}{ }^{2}$ \\
Washing length & $3 \mathrm{ft}$ & $3 \mathrm{ft}$ \\
Pumping rate & $5 \mathrm{bbl} / \mathrm{min}$ & $15 \mathrm{bbl} / \mathrm{min}$ \\
Slot flow velocity & $1.70 \mathrm{ft} / \mathrm{sec}$ & $5.10 \mathrm{ft} / \mathrm{sec}$ \\
\hline
\end{tabular}


Jet Entrainment

Flow from the slot will impinge directly on the wall of the open hole with the flow dividing at a central stagnation 1 ine corresponding to the center of the jet. Reynolds number of the jet will be greater than 3000 , so that flow from the jet will be turbulent, similar to that shown in Figures $3-30$ and $3-31$. As the jet moves from the slot to the wall, fluid will be entrained by turbulent mixing between the jet and the surrounding medium. This entrainment will cause momentum exchange between the jet and the relatively stagnant flow so that the jet core velocity will be reduced. Let us next estimate this reduction.

In the region close to the jet, the transverse velocity will be given approximately by the relation

$$
\mathrm{v} \cong \frac{U_{\mathrm{m}}}{\mathrm{I} 6}
$$

where

$$
U_{m}=\text { maximum jet velocity }
$$

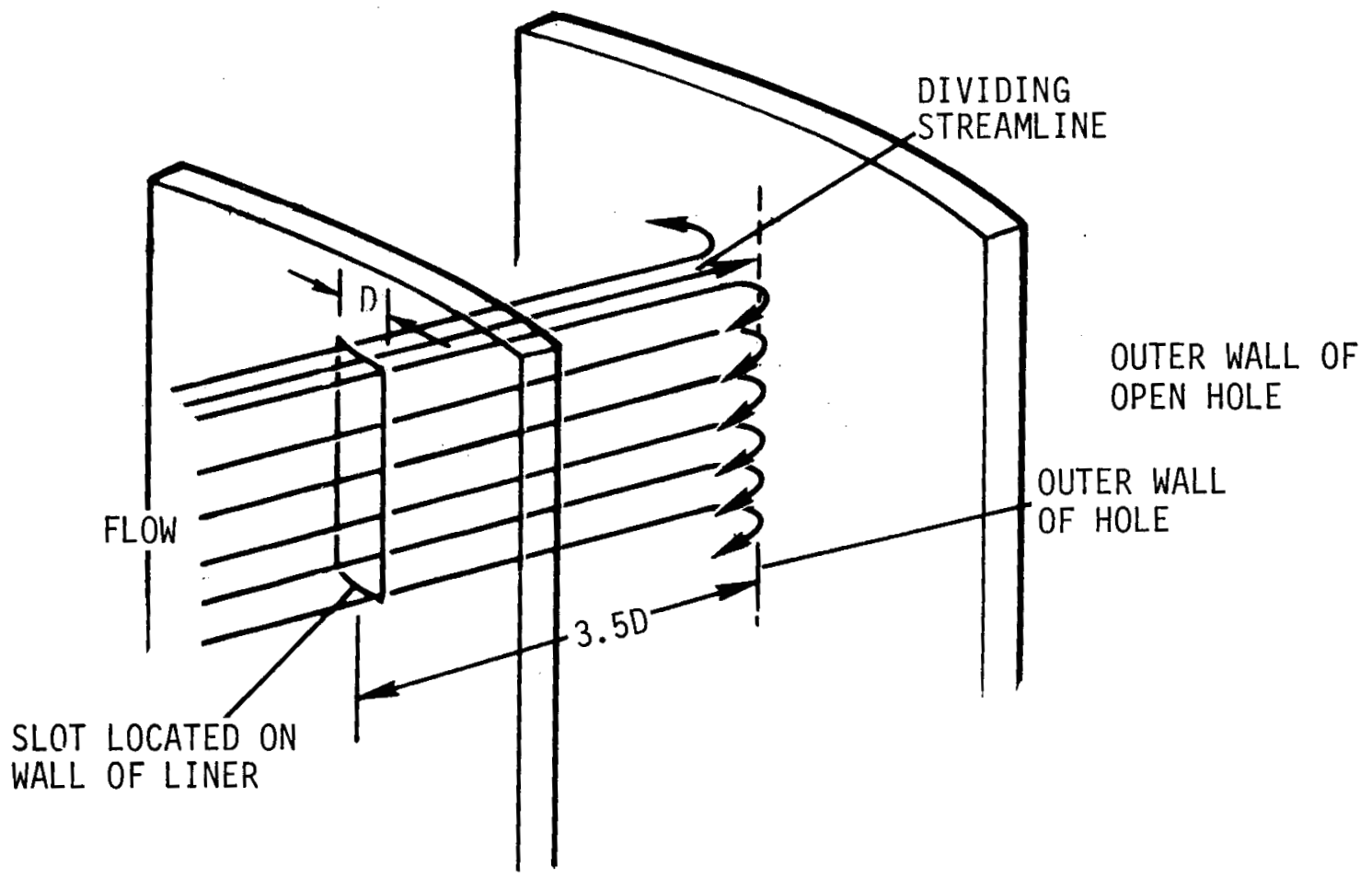

FIGURE 3-30. - Flow pattern from slot jet impinging on open hole wall. 


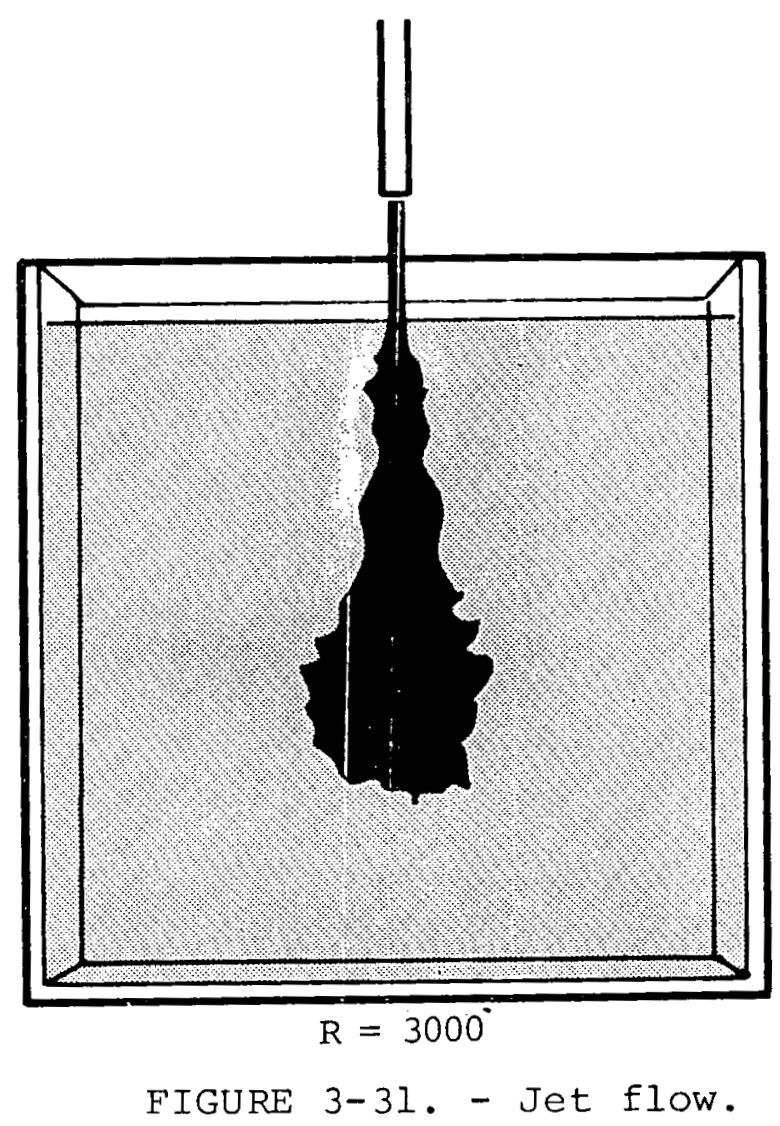

The decrease in axial velocity along the two-dimensional jet is given in Schlichting (이) as

$$
\mathrm{U}=\mathrm{U}_{\mathrm{S}}\left(\frac{\mathrm{x}}{\mathrm{S}}\right)^{-1 / 2}
$$

where the velocity $U_{S}$ is measured at" a characteristic distance $S$ from the jet exit.

If we divide the numerator and denominator by the slot width D, we get the expression

$$
\frac{\mathrm{U}}{\mathrm{U}_{\mathrm{S}}}=\left(\frac{\mathrm{X} / \mathrm{D}}{\mathrm{S} / \mathrm{D}}\right)^{-1 / 2}
$$

where

$$
\begin{aligned}
& X / D=\text { dimensionless distance from slot } \\
& S / D=\text { dimensionless characteristic distance }
\end{aligned}
$$


We would expect that the characteristic distance $S$ would not be equal to the slot width, but to some multiple thereof. In the next subsection, an experiment is described in which the dynamic pressure $\mathrm{J} / 2 \mathrm{\rho} \mathrm{U}^{2}$ is measured at the wall. From this measurement and knowledge of the jet flow rate, the characteristic distance can be determined.

\section{Shear Stress Near Stagnation Point}

The jet flow from the slot impinges the opposite wall and divides. A schematic representation of the flow is shown in Figure 3-32. From the stagnation point, the flow can be considered laminar and will have a velocity profile as shown.

The potential velocities in the region of the stagnation point are given by

$$
\begin{aligned}
\mathrm{U} & =\mathrm{ax} \\
\mathrm{V} & =-\mathrm{ay}
\end{aligned}
$$

where a denotes a constant.

If we assume that there is little decay in the velocity from the slot to a distance of 0.1 in. from the wall, we can evaluate the constant a.

$$
\mathrm{a}=\mathrm{V} / \mathrm{y}=\frac{1 \mathrm{ft} / \mathrm{sec}}{0.1 \mathrm{in} .}=10 \frac{\mathrm{ft} / \mathrm{sec}}{\mathrm{in}}
$$

This value of a can be used to calculate the velocity distribution in the developing boundary layer on a wedge having a 180 deg included angle. The solution is from Schlichting (80) (Chapter IX, Page 150).

$$
\begin{aligned}
\eta & =\left(\frac{2 U}{v x}\right)^{1 / 2} y \\
& =\left(\frac{2}{v} a\right)^{1 / 2} y
\end{aligned}
$$

From the above expression and Figure 3-33, Table 3-13 was constructed. 

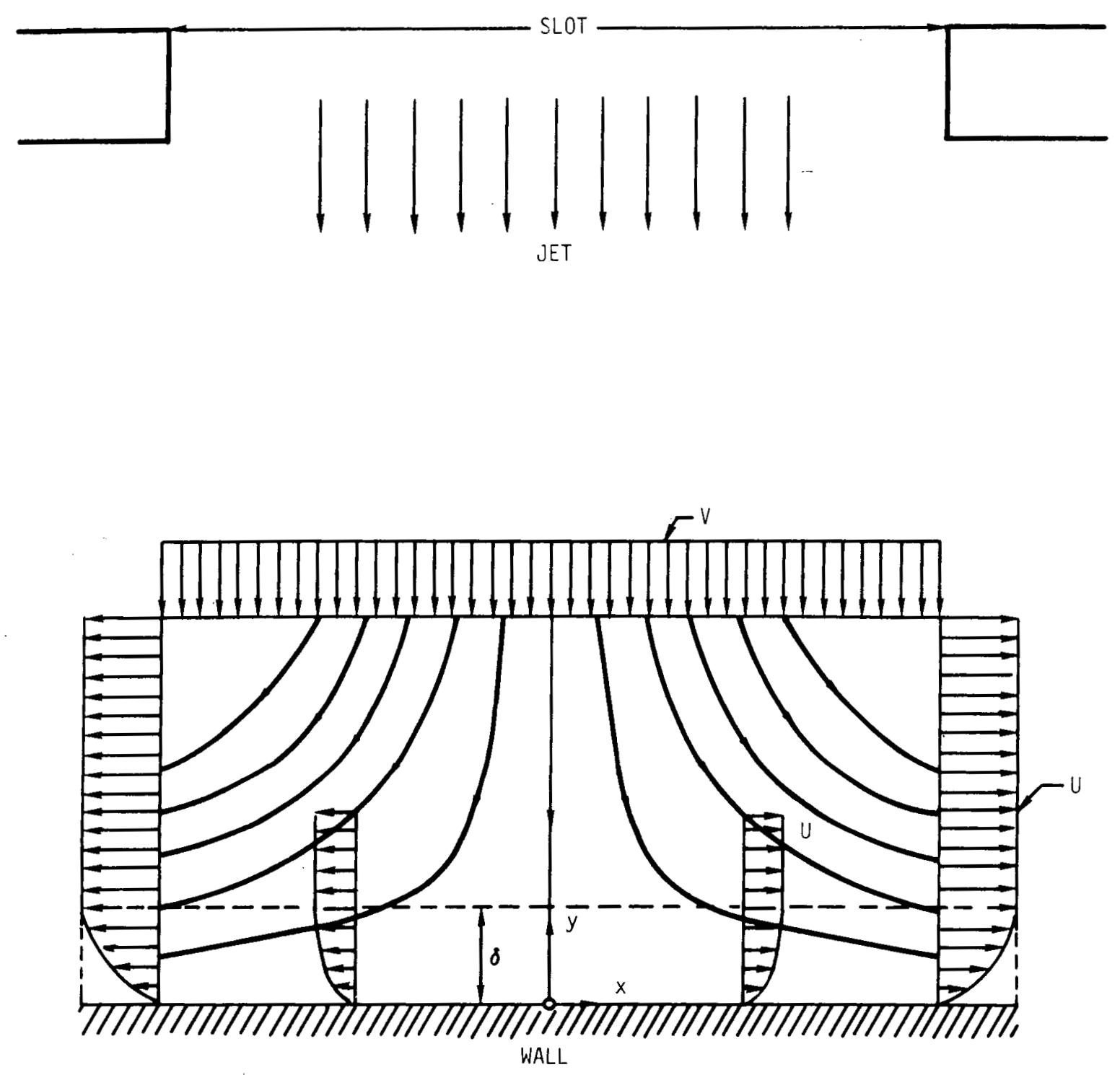

FIGURE 3-32. - Stagnation in plane flow. 


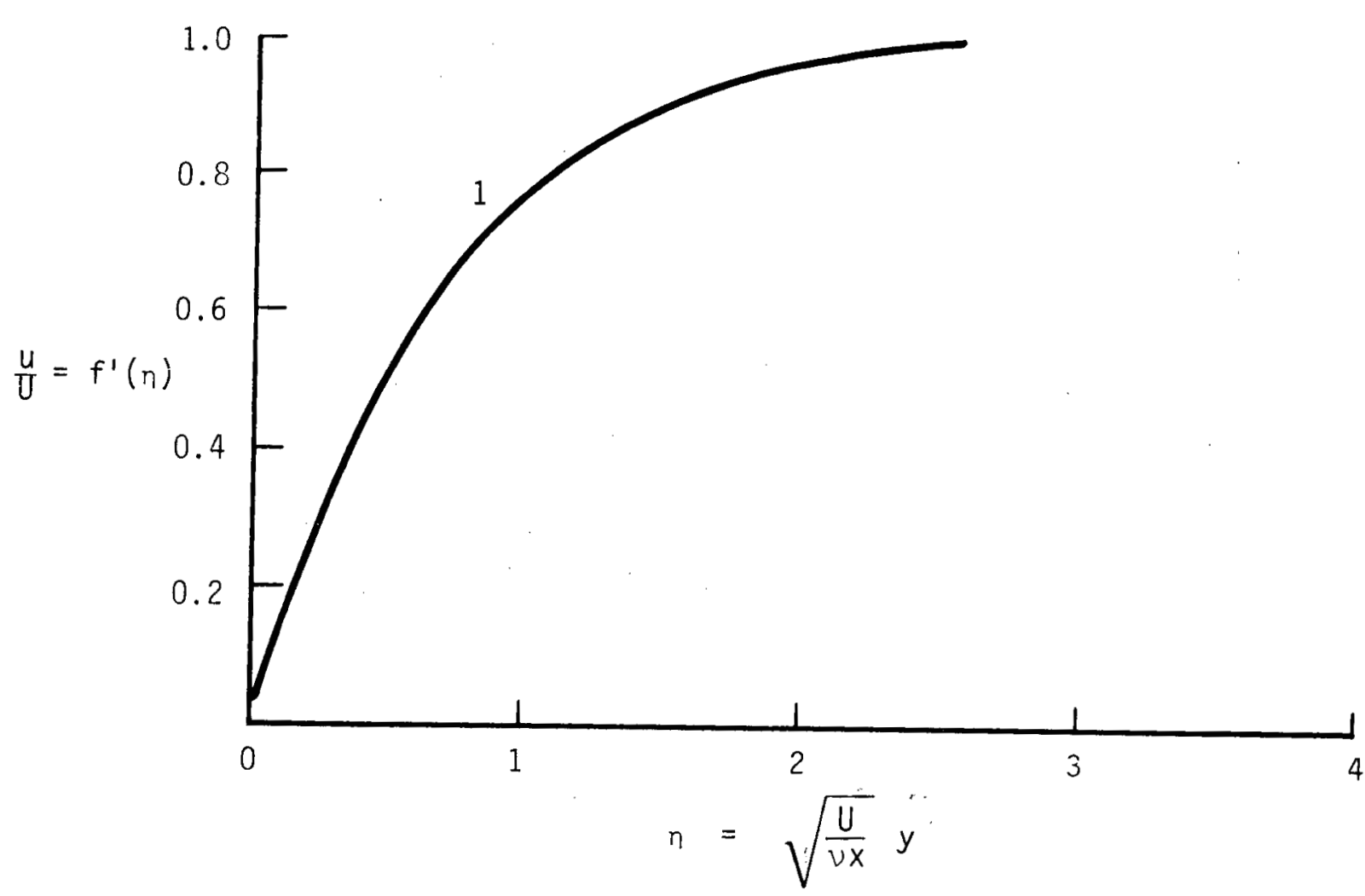

HIGURE 3-33. - Velocity distribution in the laminar boundary layer on a flat wall in the vicinity of the stagnation point.

TABLE 3-13. - Velocity distribution at $x=0.1 \mathrm{in.}$ ( $\mathrm{U}_{\text {jet }}=1 \mathrm{ft} / \mathrm{sec}$ )

\begin{tabular}{|l|l|l|l|}
\hline$y$ (in.) & $n$ & $u / U$ & $u(f t / s e c)$ \\
\hline 0 & 0 & 0 & 0 \\
0.0005 & 0.173 & 0.25 & 0.25 \\
0.001 & 0.346 & 0.5 & 0.5 \\
0.002 & 0.693 & 0.75 & 0.75 \\
\hline
\end{tabular}


From this velocity profile, the shear stress could be calculated

$$
\begin{aligned}
& \tau \approx \mu \frac{\Delta u}{\Delta y} \\
& \tau=6 \times 10^{-4} \quad 1 \mathrm{~b} / \mathrm{in} .^{2}
\end{aligned}
$$

Shear Stress in Region Away from Jet

For the region away from the jet, the shear stress was estimated using a force balance between the pressure drop and shear:

$$
\Delta \mathrm{P} \mathrm{A}_{\text {annulus }}=\tau_{\text {shear }} \pi\left(D_{0}+D_{i}\right) L
$$

The pressure drop was calculated for 4-in. lengths of pipe, to include one slotted and one unslotted section of pipe. It was assumed that the volume flow rate at all the slots was the same. Thus, the velocity in the annular space increased uniformly from the bottom to top slot row (15 maximum).

The pressure drop was calculated using the familiar:

$$
\Delta \mathrm{P}=4 \pm \frac{\mathrm{L}}{\mathrm{D}_{\mathrm{e}}} \rho \frac{\mathrm{V}^{2}}{2}
$$

where, for the turbulent region in question, the friction factor can be expressed as:

$$
f=\frac{0.157}{R_{e}^{0.323}}
$$

where the Reynolds number is based on the hydraulic diameter $D_{e}=D_{0}-D_{i}$.

For a 4-in. length of liner (one slotted section, one unslotted section), the shear stress can then be expressed as:

$$
\tau=0.00101\left(\frac{\mu}{\rho D_{e}}\right)^{0.323} \mathrm{v}^{1.677}
$$


Here, $V$ is the velocity in the annular space. Results are shown in Table 3-15 for slot velocities of 1 and $6 \mathrm{ft} / \mathrm{sec}$.

The above analysis ignores the effect of the increased shear on developing boundary layers. It can be shown that, in the entrance regions of pipes where the boundary layer is developing, the pressure gradient and the shear stress are approximately an order of magnitude greater than in fully developed flow. Even following the application of this factor to Table 3-14, the shear stresses are still quite small.

It is concluded from this exercise that the cleaning effect of the jets will be at best local and that the majority of the hole wall will remain covered with mud cake.

\subsubsection{Experimental Evaluation of Slotted Liner Washing Methods}

In order to evaluate the potential effectiveness of slotted liner washing, flow emanating from a slotted liner and impinging on an open wall was simulated. The experimental apparatus of Figure 3-34 was constructed. A series of $0.250-i n$. wide by 2-in. long slots were machined into the outer diameter of a 4.5-in. OD PVC plastic pipe. The hole pattern was selected such that the jet velocity out of each slot would be approximately $2 \mathrm{ft} / \mathrm{sec}$ at the maximum measurable pumping rate - $30 \mathrm{gal} / \mathrm{min}$. The hole pattern is shown in Figure $3-35$.

\section{TABLE 3-14. - Average shear stress between slotted liner and wall (7 and 8.75 in., respectively)}

$\begin{array}{ccc}\text { Slot } & \begin{array}{c}\text { Slot velocity } \\ 1 \mathrm{ft} / \mathrm{sec}\end{array} & \begin{array}{c}\text { Slot velocity } \\ 6 \mathrm{ft} / \mathrm{sec}\end{array} \\ 1 & 8.0 \times 10^{-7} & 1.6 \times 10^{-5} \\ 4 & 8.0 \times 10^{-6} & 1.6 \times 10^{-4} \\ 8 & 2.5 \times 10^{-5} & 5.2 \times 10^{-4} \\ 15 & 7.3 \times 10^{-5} & 1.5 \times 10^{-3}\end{array}$




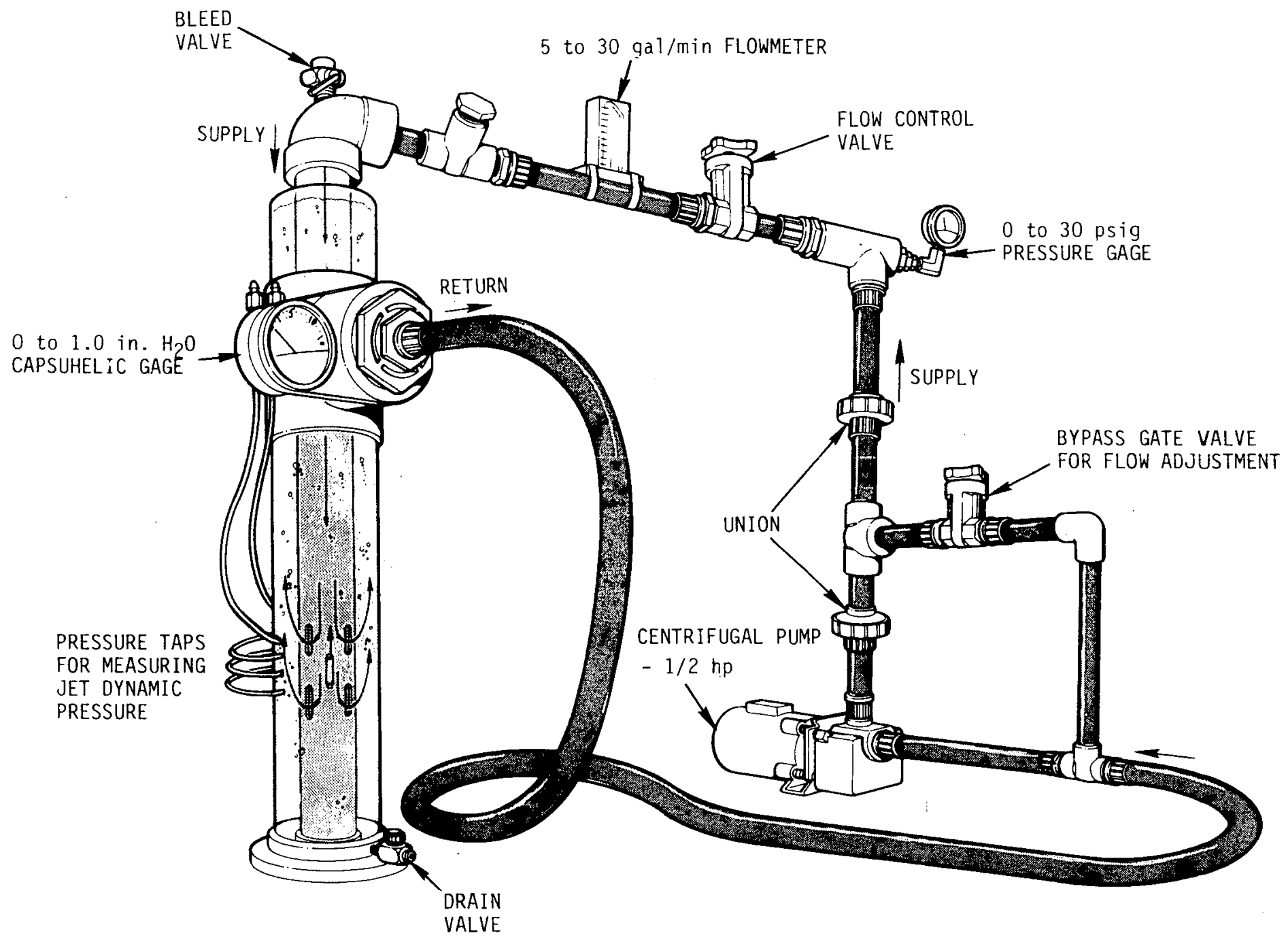

FIGURE 3-34. - Experimental test apparatus. 


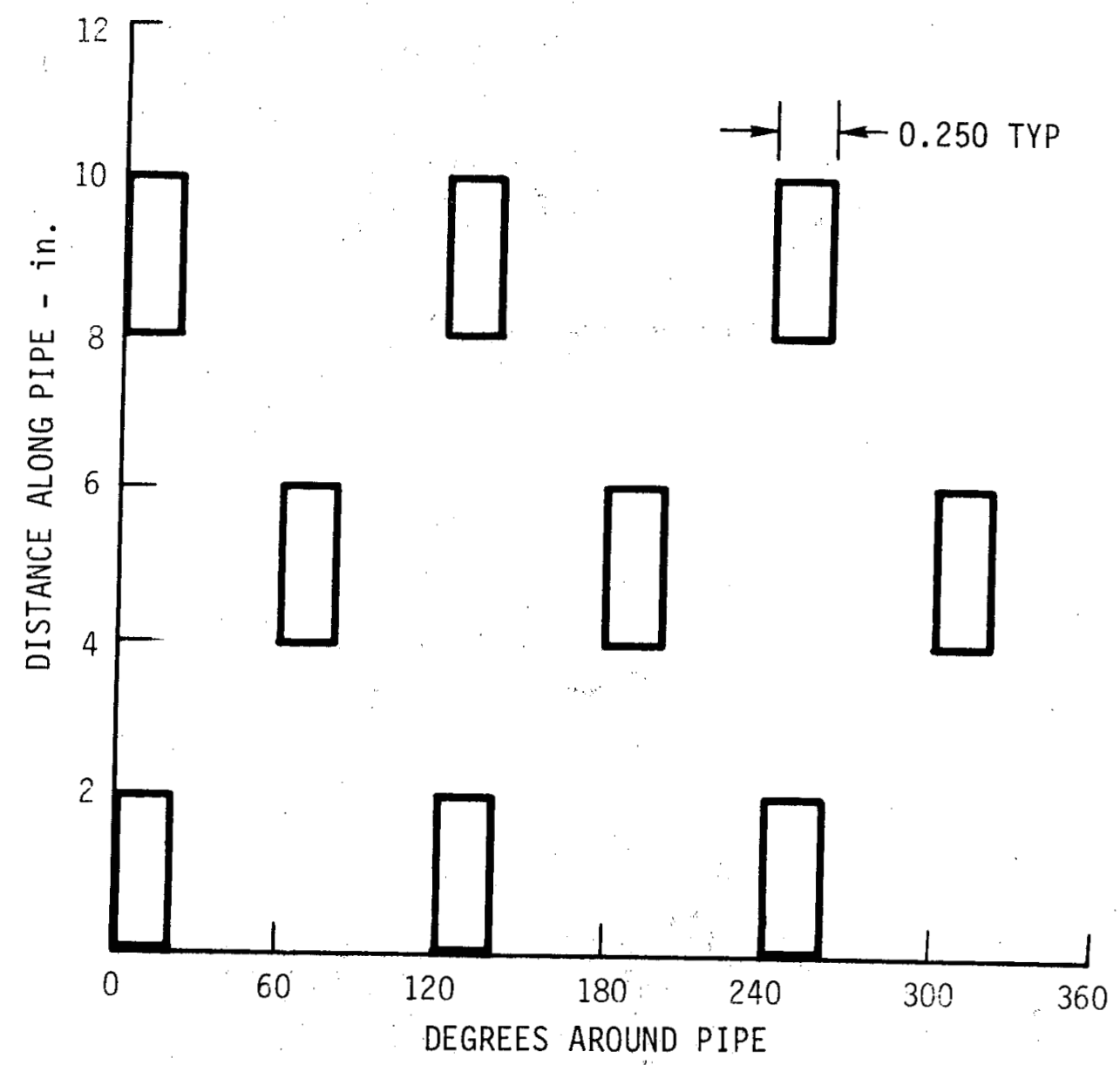

FIGURE 3-35. - Hole pattern in 4.5-in. pipe for slotted liner washing tests. 
The 4.5-in. PVC pipe was capped at the bottom and water was pumped into the top from a $1 / 2$ hp centrifugal Teel Pump. Flow from the pump was measured by a Model CF-300 Flowmeter manufactured by $\mathrm{Bl}$ ue white Industries. Flow rate was regulated by throttling a flow control valve so that the flow rate could be varied over the range of flow rates of interest.

Flow from the slot impinged on the inside diameter of a 6-in. pipe. The inside diameter measured 6.62 in., so that the gap between the 4.5-in. OD pipe and the 6-in. pipe was 1.06 in., similar to that found between a 7-in. liner and an 8-3/4-in. open hole $(0.875$ in.). The return flow was collected at a 6-in. pipe tee which permitted the flow to return to the suction side of the pump while allowing the central slotted pipe to pass through the tee.

The central slotted pipe was designed to be adjustable so that a series of pressure taps located in the outer wall could be positioned relative to the opposing slots. The outer wall was instrumented with 10 pressure taps which could be connected two at a time to a 0 to 1.0 in. water differential pressure gauge manufactured by Dwyer Instruments.

For the total slot area of $4.5 \mathrm{in.}$ at a flow rate of $25 \mathrm{gal} /$ min, flow velocity out of the slot is

$$
\mathrm{V}_{\text {slot }}=1.78 \mathrm{ft} / \mathrm{sec}
$$

which corresponds very closely to the slotted liner Case No. I, where $5 \mathrm{bbl} / \mathrm{min}$ are pumped through $3 \mathrm{ft}$ of slots spaced at 16 slots per foot of the same cross-section as fabricated ( 2 in. by 0.25 in.).

The maximum pressure due to jet flow which can be measured on the wall opposing the jet is the dynamic pressure

$$
\mathrm{q}_{\max }=1 / 2 \rho \mathrm{U}_{\max }^{2}
$$

where $U_{\max }$ is determined from the flow rate to be $1.78 \mathrm{ft} / \mathrm{sec}$. Substituting values gives a maximum dynamic pressure

$$
\mathrm{q}_{\max }=0.558 \text { in } \cdot \mathrm{H}_{2} \mathrm{O}
$$


The reader must note that this is an extremely low pressure.

By connecting the pressure tubes to the differential pressure gauge such that the high-pressure port faced the jet and the lowpressure port was located midway between jets, the pressure distribution shown in Figure 3-36 was measured. Note that the maximum jet dynamic pressure was measured to be 0.51 in. $\mathrm{H}_{2} \mathrm{O}$ at the center of the jet. At the base of the jet along the centerline the dynamic pressure was essentially zero due to the upturn in the jet.

The dynamic pressure ratio between the jet exit and the wall can be written as

$$
\frac{q_{\text {wall }}}{q_{\text {max }}^{\text {theoretical }}}=\left(\frac{U_{\text {wall }}}{U_{\text {jet }}}\right)^{2}=\frac{S / D_{\text {wall }}}{X / D}
$$

Since the wall dynamic pressure ratio is known, we can determine the characteristic distance as

$$
\begin{aligned}
& S / D=\left(\frac{X}{D}\right) \frac{q_{\text {wall }}}{q_{\text {max }}} \text { theoretical } \\
& \frac{S}{D}=3.88 \text { slot diameters }
\end{aligned}
$$

This is the characteristic distance for this jet at these operating conditions of the test.

In order to evaluate the effect of the slot on cleaning, a series of qualitative tests was conducted to determine the effect of the jet on "mud" cleaning from the sidewall. As was to be expected, the results depend greatly on the strength of the mud.

In order to simulate mud on the wall, a base of patching plaster was bonded to a wire mesh corresponding to the shape of the inside diameter of the 6-in. pipe. The thickness of the plaster was approximately $1 / 8$ in." so that the hardened plaster would represent a uniform wall to the jet. A wire rod was attached to the wire mesh so that the wire mesh and its mud base could be lowered into the test chamber, opposite the slotted liner (see Figure 3-37). 


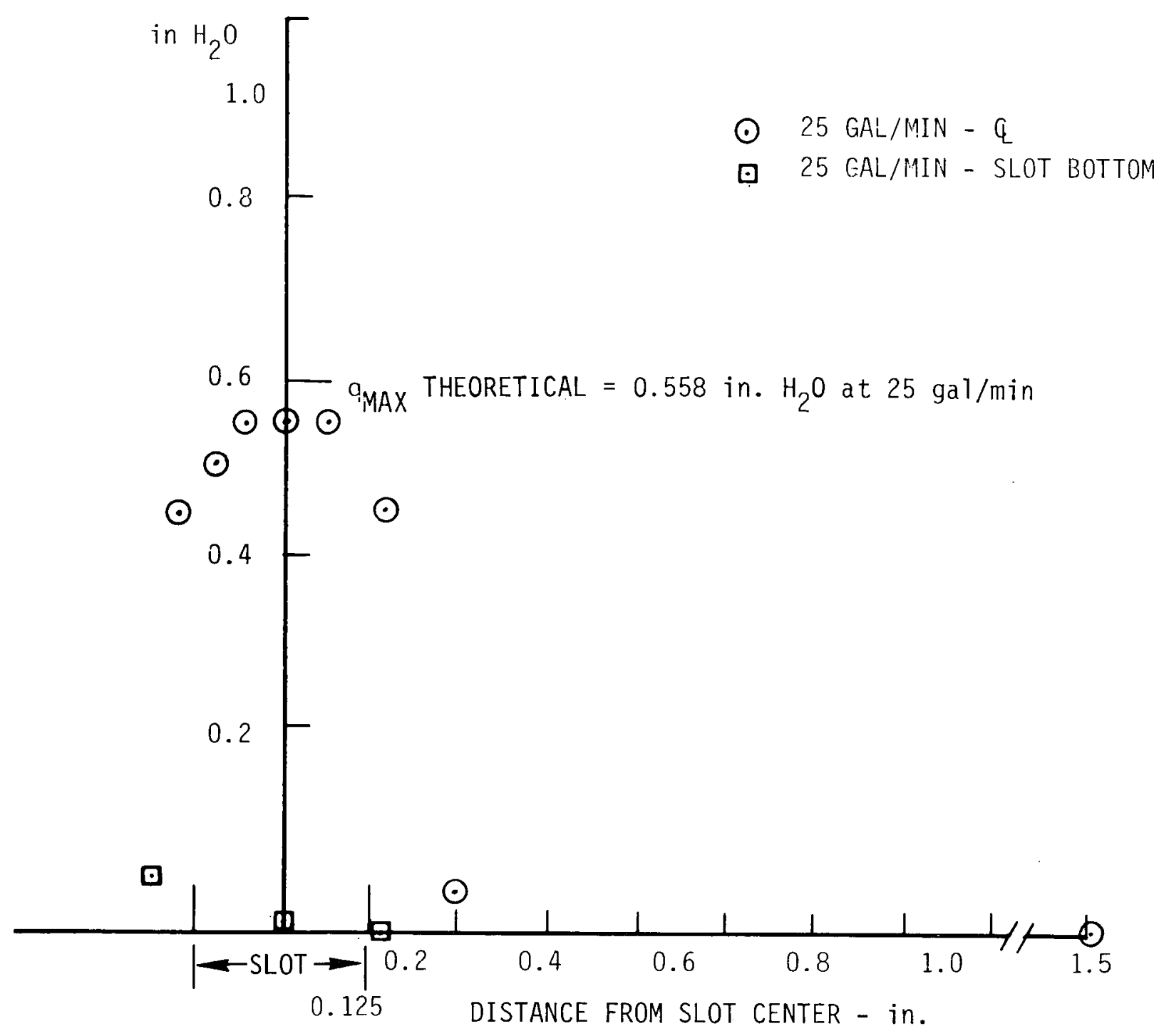

FIGURE 3-36. - Jet dynamic pressure distribution. 


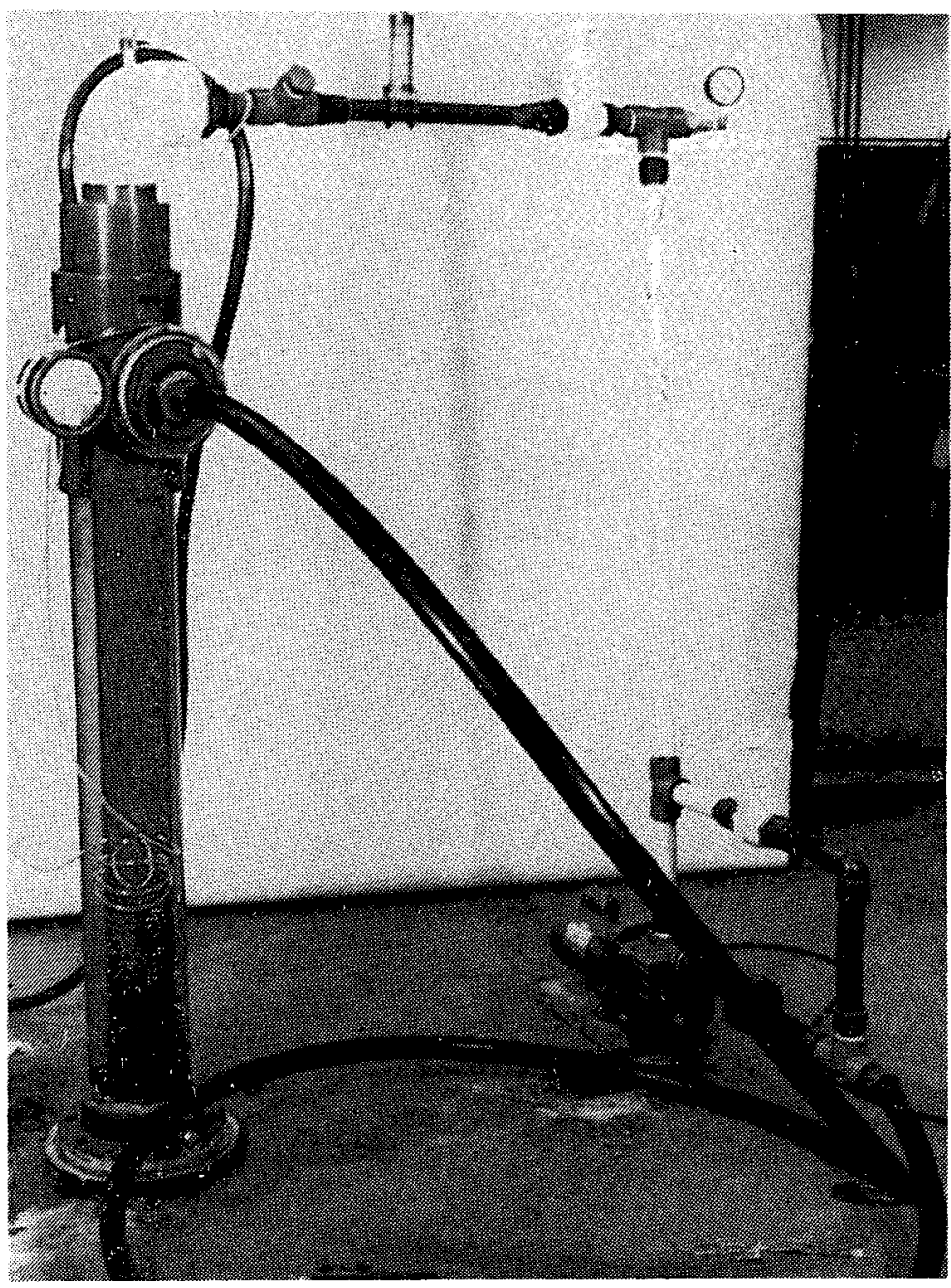

FIGURE 3-37. - Photograph of plaster-mud erosion test setup.

Patching plaster was mixed in a cup and layered on the firm plaster-wire mesh base. Simulated mud thickness was approximately $1 / 4$ in. thick by 2 in. long along the holding fixture.

Three separate tests were conducted with "mud" of different hardness. Unfortunately, we had devised no consistent means to quantify hardness of the quick-drying plaster. The first specimen which was inserted into the test apparatus was quite soft (see Figure 3-38a). After $5 \mathrm{~min}$ of exposure to the $1.78 \mathrm{ft} / \mathrm{min}$ jet, the sample completely washed off the base (see Figure 3-38b).

The second test sample was prepared (Figure 3-39a) and allowed to set until slightly firmer. After $5 \mathrm{~min}$ of jet washing, a groove of material had been removed from the sample (see Figure 3-39b), which was otherwise intact.

The balance of the sample hardened below the surface layer and remained intact outside of the near field of the slotted jet. 


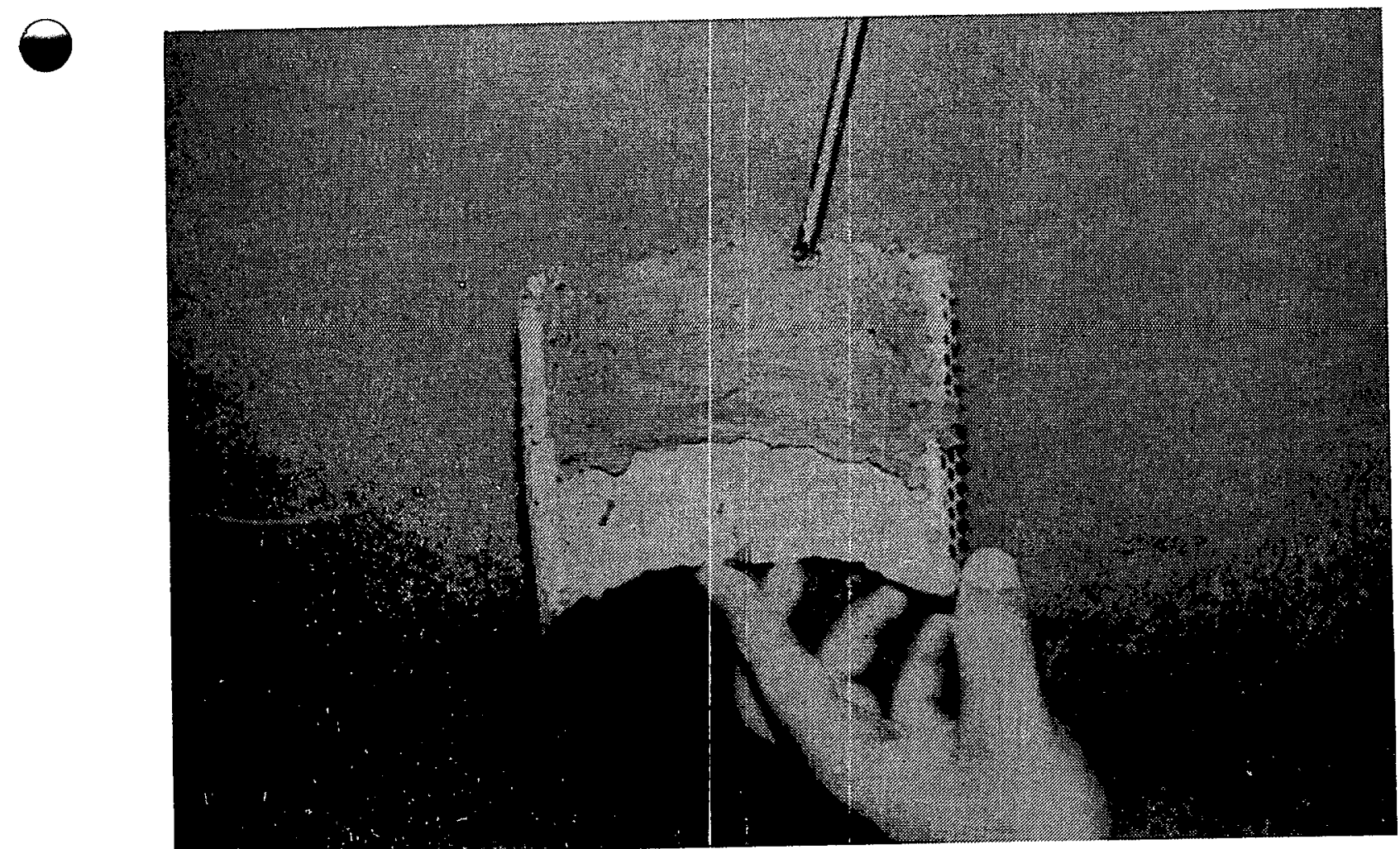

a) BEFORE TESTING

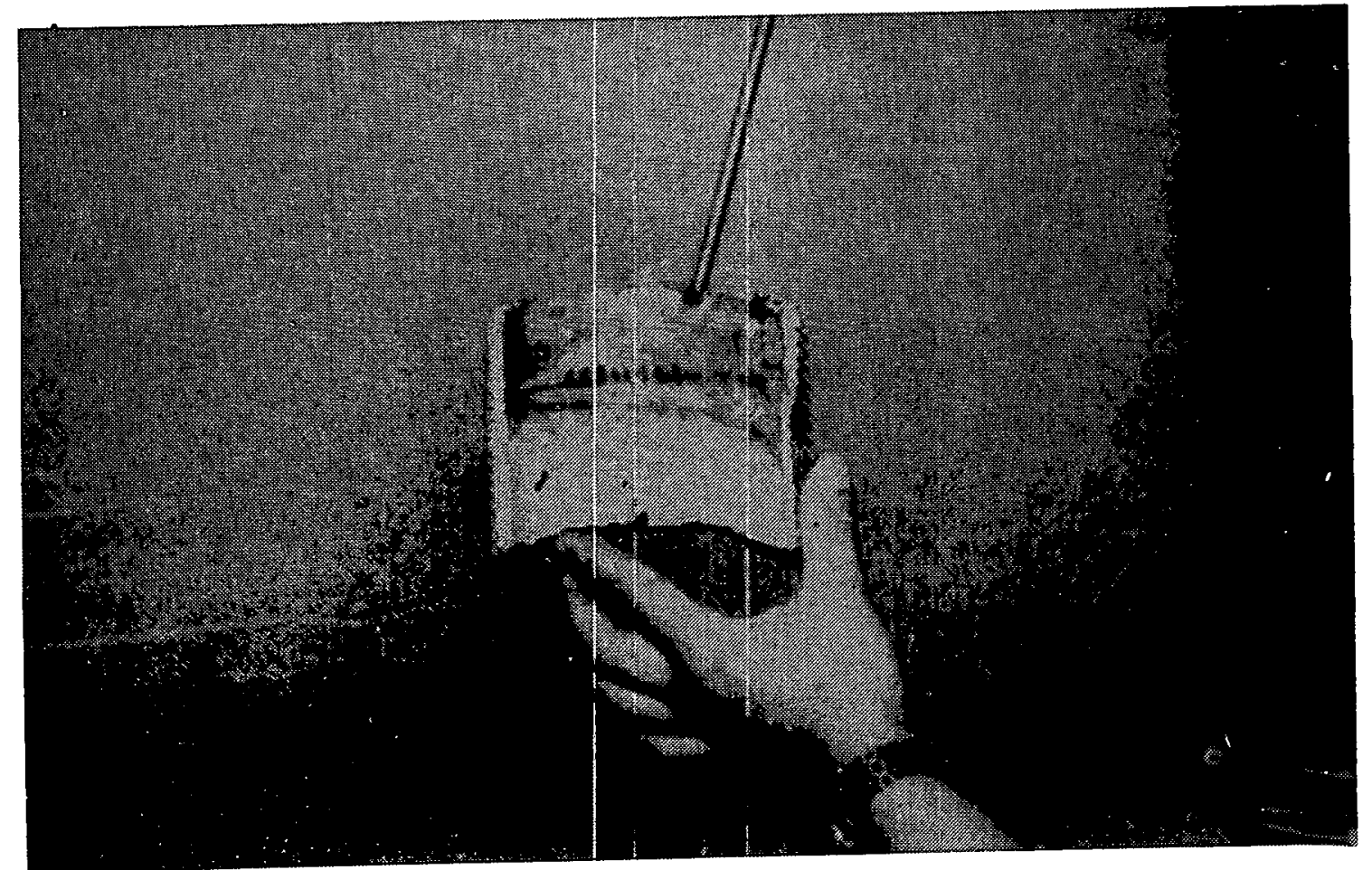

b) AFTER TESTING - FIGURE 3-38. - Testing of soft plaster-mud samples at slot velocity
of $1.7 \mathrm{ft} / \mathrm{sec}, 5$-min sample explosure. 


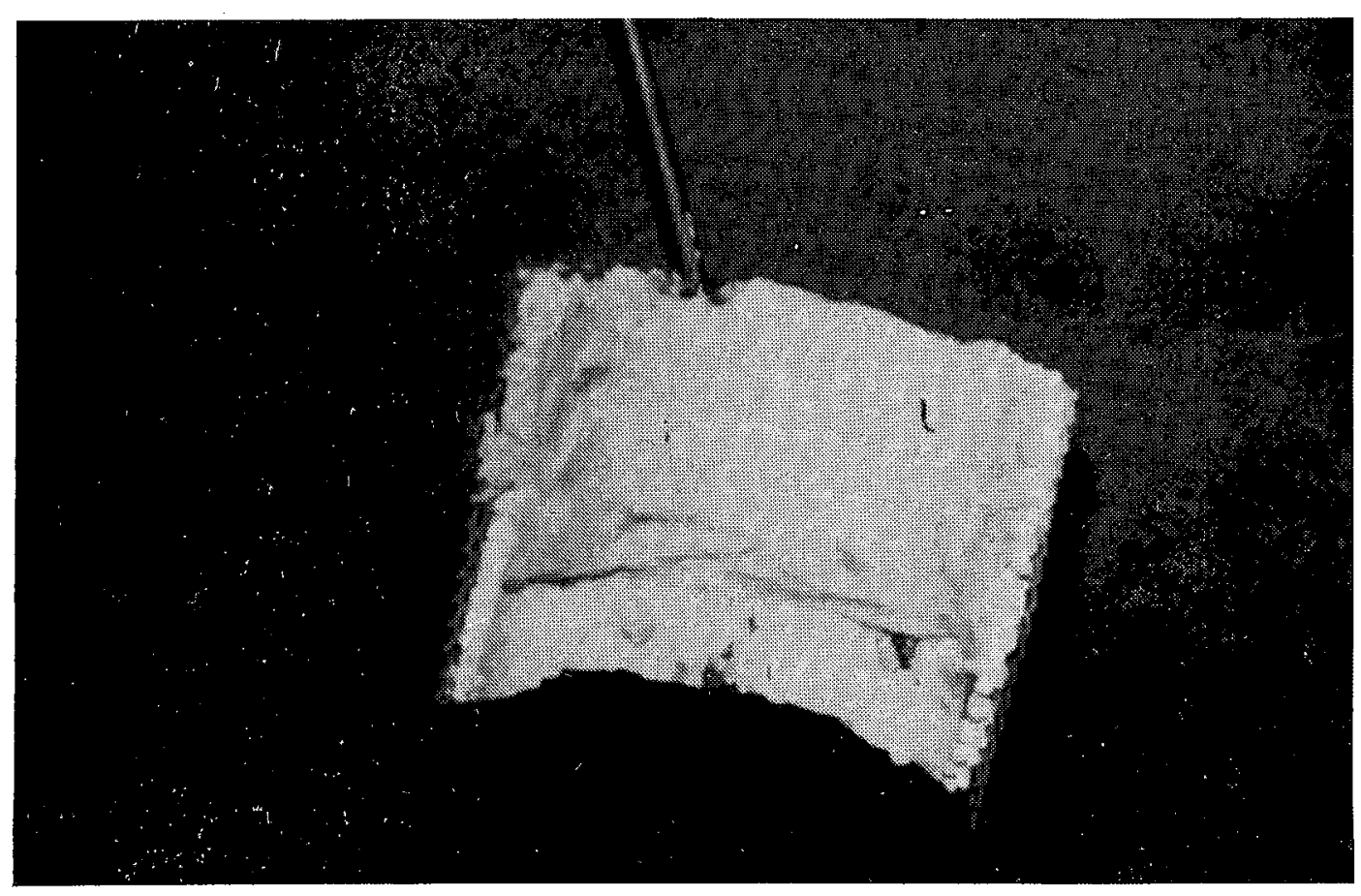

a) BEFORE TESTING

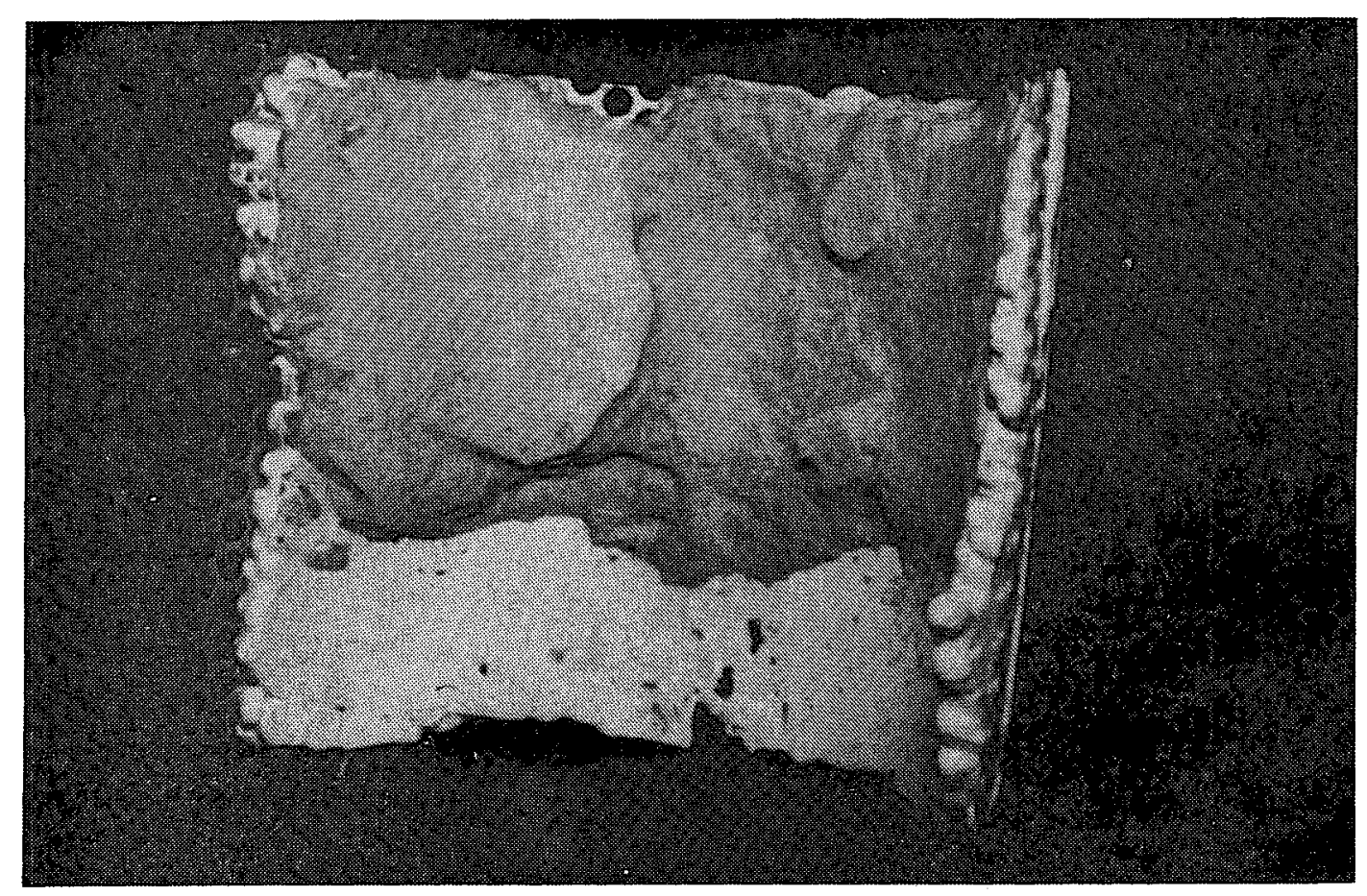

b) AFTER TESTING

FIGURE 3-39. - Testing of medium-hard plaster-mud samples at slot velocity of $1.7 \mathrm{ft} / \mathrm{sec}$, 5-min sample exposure. 
The third sample was prepared and let harden slightly longer. Again, the sample was placed opposite the jet and washed for $5 \mathrm{~min}$. No material was visibly removed." After successive 5-min washings up to $15 \mathrm{~min}$, nothing happened. See Figure 3-40a and 3-40b for before and after photographs.

\subsubsection{Conclusions}

Our conclusions are presented below:

a. It was assumed in subsection 3.3 .3 that there is very little jet spreading between the slotted liner and the side of the borehole. This assumption has been verified experimentally.

b. The shear forces exhibited by the jet in the vicinity of the wall are small. For a jet speed of $1 \mathrm{ft} / \mathrm{sec}$ the shear stress varies linearly. from zero at the stagnation point to $6 \times 10^{-4} \mathrm{lb} / \mathrm{in} .2$ at a distance 0.1 in. away from the stagnation point. The shear stress varies linearly with velocity.

c. The shear forces exerted on the entire wall in those regions in line with the liner slots were estimated using Moody pressure drop correlations modified to include the effects of boundary layer development. For 15 rows of slots ( $5 \mathrm{ft}, 3$ slot rows per foot) and for a slot velocity of $1 \mathrm{ft} / \mathrm{sec}$, the shear stress varied from $8 \times 10^{-6} 1 \mathrm{~b} /$ in. 2 near the first slot row to $8 \times$ $10^{-4} \mathrm{lb} / \mathrm{in}^{2}$ near the fifteenth slot row. The shear stress varied with jet velocity to the 1.68 th power.

d. Mud removal by a jet of water from a slotted liner will vary greatly with the consistency of the mud. At pumping conditions of $5 \mathrm{bbl} / \mathrm{min}$ over a 3 -ft section of 16 slots per foot ( 2 in. $\times 0.5$ in.), slot flow velocity is barely adequate to remove mud directly in front of the jet. At higher pumping rates ( $15 \mathrm{bbl} / \mathrm{min})$, over a shorter section of material to be washed ( $1 \mathrm{ft}$ ), jet impingement pressure will not exceed $3.79 \mathrm{lb} / \mathrm{in}^{2}$ opposite the jet.

\subsubsection{Recommendations}

Based on prior analysis and experimentation, it is unlikely that slotted liner washing is an effective means of cleaning mud from the open hole wall. Shear stresses exerted everywhere on the mud cake on the hole wall are small. The width of the jet as it impacts the wall is little wider than the slot from which it emanates. This method of cleaning is obviously not the most effective method of cleaning the hole wall. 


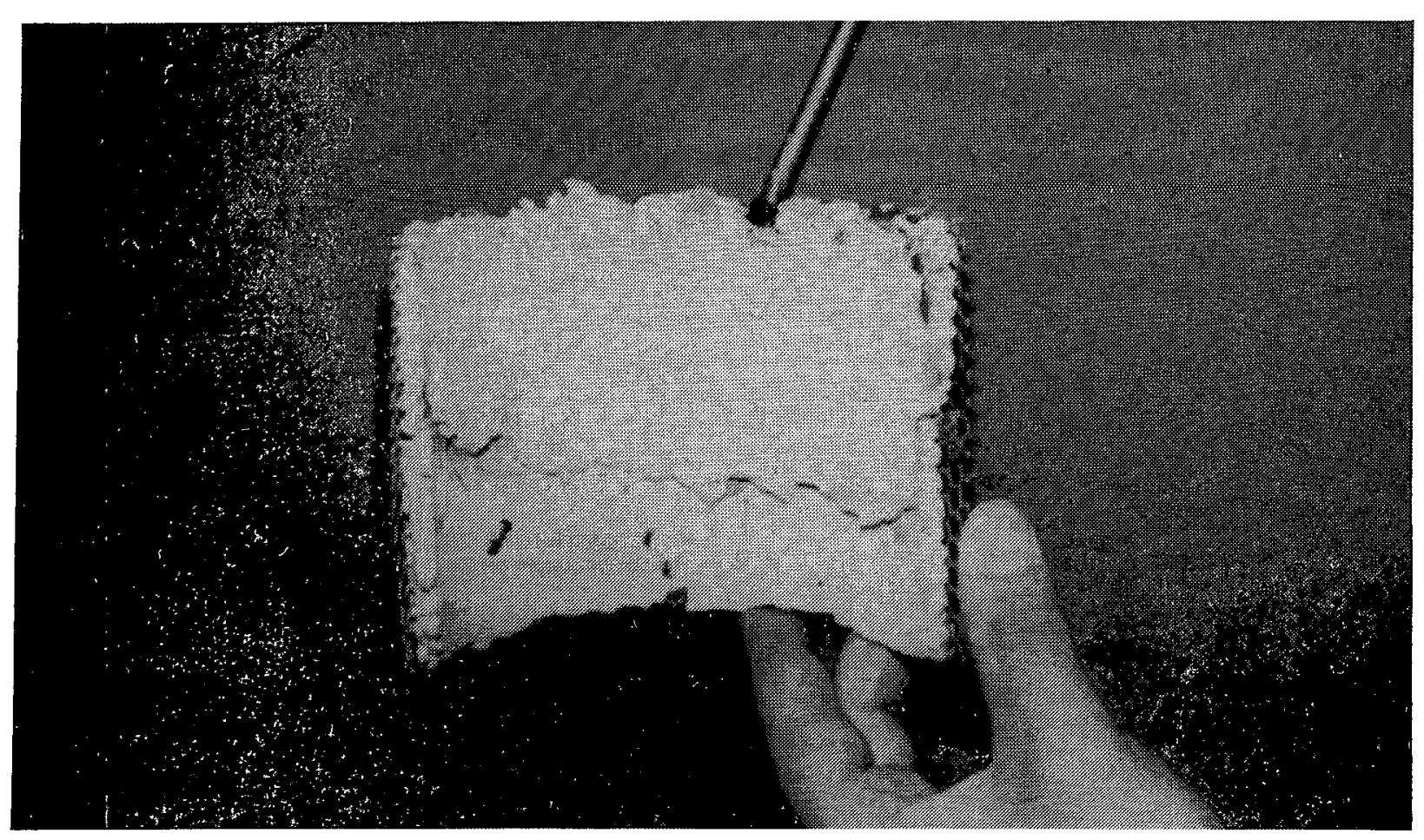

\section{a) BEFORE TESTING}

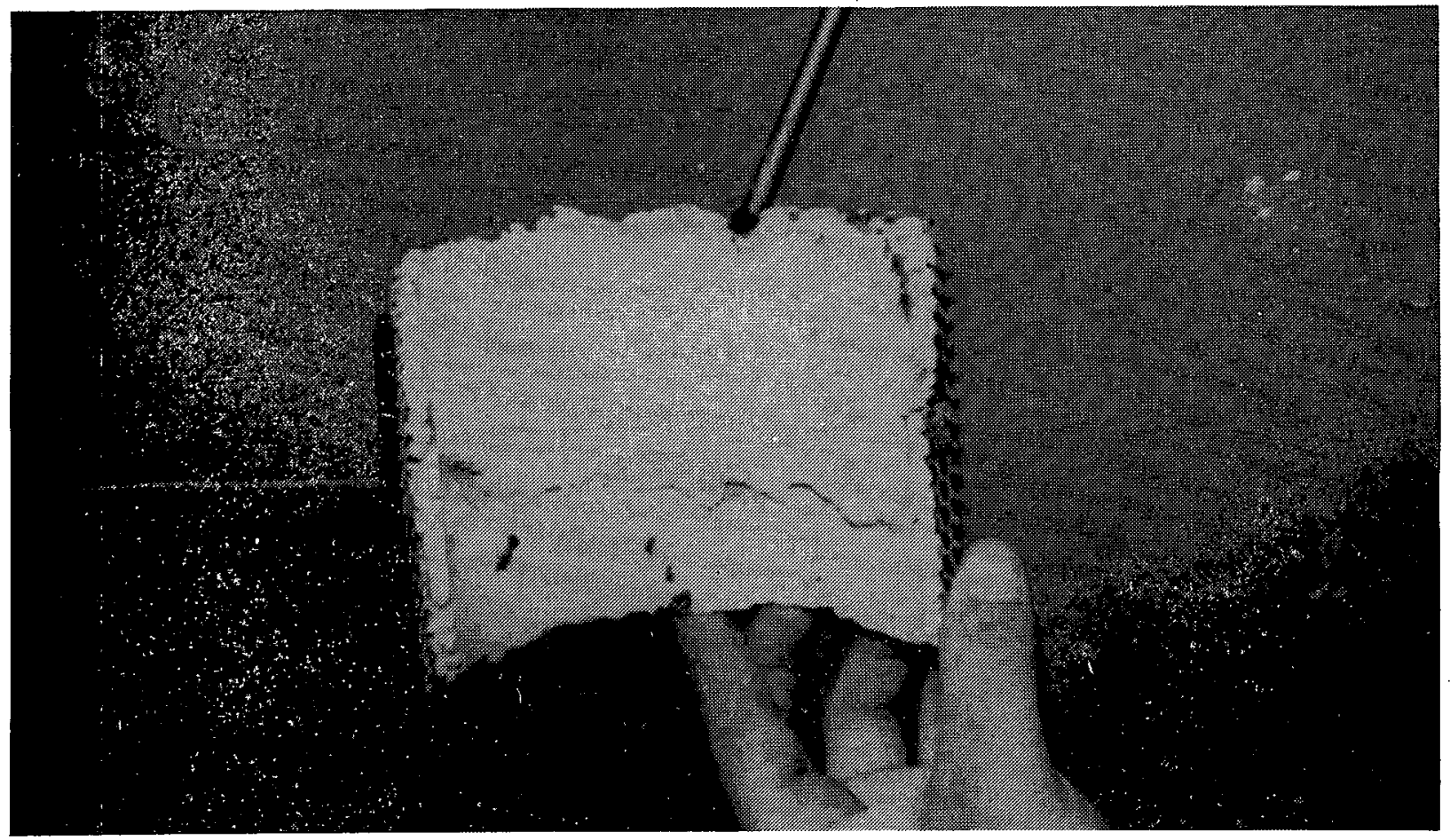

b) AFTER TESTING

FIGURE 3-40. - Testing of firm plaster-mud samples at slot velocity of $1.7 \mathrm{ft} / \mathrm{sec}, 15-\mathrm{min}$ sample exposure. 
It is recommended that alternate means of mud cleaning be investigated, such as pulsitile flow or agitated oscillatory flow between the outer and inner hole. Present estimates are that only one to two slot widths of wall are being cleaned by the jets. 


\section{REFERENCES}

\subsection{References for Subsection 2.1}

1. Snyder, R.E., "Geothermal Well Casing Design: A Study of Failure Modes and Cementing Limitations," prepared for Sandia Laboratories under Contract 13-2297, Albuquerque, NM, May 1979.

2. Dominguez, B.A. and G.S. Garcia, "Geothermal Drilling in Cerro Prieto," International Conference on Geothermal Drilling and Completion Technology; Albuquerque, NM, 21 to 23 January 1981.

3. Suman, G.O. Jr. and R.C. Ellis, World Oil's Cementing Handbook, Gulf Publishing Co., Houston, TX, 1977 .

\subsection{References for Subsection 2.2}

4. Snyder, R.E., "Typical Well Completions and Downhole Environments for Major Western U.S. Geothermal Developments," a study in support of the Sandia/FMA contract, January 1981.

5. Tixier, M.P., G.W. Loveless and R.A. Anderson, "Estimation of Formation Strength from Mechanical Properties Log," Journal of Petroleum Technology, March 1975.

6. Coates, G.R., "Log Derived Mechanical Properties and Rock Stress," SPWLA, Twenty-Third Annual Logging Symposium, 8 to 11 July 1980 .

7. Schlumberger, Communication with company representative and review of product Iiterature (Service Catalog), Houston, TX, March 1981.

8. Suman, G.O.,Jr., World Oil's Sand Control Handbook, Part l, Gulf Publishing Co., Houston, TX, 1975.

9. Halliburton Services, Technical Data Book - Sand Control Methods, Duncan, OK, $1 \overline{974 .}$

10. Santrol Products, Inc., Communication with company representative and review of product literature, Houston, TX, March 1981.

11. B\&W, Inc., Composite Catalog of Oil Field Equipment and Services, Vol 1, Gulf Publishing Co., Houston, TX, 1980-1981. 
12. Lynes, Composite Catalog of Oil Field Equipment and Services, Vol 3, Gulf Publishing Co., Houston, TX, 1980-1981

13. Texas Brine, Communication with company representative, Houston, TX, March 1981.

14. Mayco Wellchem, Inc., Communication with company representative, Houston, TX, March 1981.

15. American Cynamid Co., Communication with company representative and review of product literature (Oilfield chemicals), Houston, TX, March 1981.

16. Reed, M.G., "Gravel Pack and Formation Sandstone Dissolution During Steam Injection," Journal of Petroleum Technology, June 1980.

17. Clemtex, Ltd., Communication with company representative, Houston, TX, March 1981 .

18. Sunshine Mining Co., Communication with company representative, Kellogg, ID, March 1981.

19. Athavaley, A.S. and R.G. Sweet, "Assessment of Gravel Pack Materials for Industrial Disposal Well Applications," unpublished manuscript, Houston, TX, 1980.

\subsection{References and Industry Contacts for Subsection 2.3}

20. Rinaldi, R.E., "Comparative Cost Analyses of Scale Control Techniques," unpublished report for Sandia Laboratories prepared by Resource Technology, Inc., Tulsa, OK, March 1981.

21. Jorda, R.M., "State of the Art in Well Completion Technology as Applied to Geothermal Development," Sandia Laboratories Report No. SAND 78-7008, May 1978.

22. NL Treating Chemicals, Communication with company representative and review of product literature, Houston, TX, March 1981 .

23. Petrolite Corp.-Tretolite Div., Communication with company representative and review of product literature, Brea, CA, March 1981.

24. Hughes, B.J., Communication with company representative and review of product literature, Bakersfield, CA, March 1981.

25. Halliburton Services, Communication with company representative and review of product literature, Duncan, OK, March 198 
26. Exxon Chemicals, Review of product (Corexit) literature, Houston, TX, March 1981.

27. Hydril, Communication with company representative, Houston, TX, March 1981 .

28. Sperry-Sun, Communication with company representative, Houston, TX, March 1981.

29. Tenneco, Communication with company representative, Lafayette, LA, March 1981.

30. Sun-Energy Development, Communication with company representative, Dallas, TX, March 1981.

31. TRW-Reda Pumps, Communication with company representative, Bartlesville, OK, March 1981.

32. Peerless Pump Co., Communication with company representative and review of product literature, Houston, TX, March 1981.

33. Frank's Casing Crew and Rental Tools, Communication with company representative, Lafayette, LA, March 1981.

34. Varco Oil Tools, Composite Catalog of Oil Field Equipment and Services, Vol 4 , Gulf Publishing Co., Houston, TX, 1980-81.

\subsection{References and Industry Contacts}

35. Snyder, R.E., Typical Well Completions and Downhole Environments for Major Western U.S. Geothermal Developments, a study in support of the sandia/FMA contract, January 1981.

36. Barton, J.A., "Geothermal/Perforating and Pipe Recovery," Technical Training Course No. 3, Geothermal Resources Council, Albuquerque, NM, 20 to 21 February 1980.

37. Burkill, G.C.C., "Thermal Well Completion Design with Openhole Gravel Packed. Liners and Methods for Selective Steam Injection," Proceedings, Canadian-Venezuelan Oil Sands Symposium, Edmonton, Alberta, Canada, 1977, pp 597-608.

38. Suman, G.O.,Jr., World Oil's Sand Control Handbook, Gulf Publishing Co., Houston, TX, 1975. 


\subsection{References for Subsection 2.6}

39. Anon, API Recommended Practice Standard Procedure for Evaluation of Well Perforators, API R.P.43, Third Edition, october 1974 .

40. Thompson, G.D., "Effects of Formation Compressive Strength on Perforation Performance," API Drilling and Production Practice, 1962, p. 191.

41. Saucier, R.J. and J.F. Lands, Jr., "A Laboratory Study of Perforations in Stressed Formation Rocks," SPE 6758, 1977.

42. Parrish, D. and N.L. McCullough, "Explosives of Interest to N.L. McCullough and Glossary of Terms."

43. Barton, J.A., "Geothermal Perforating and Pipe Recovery," Technical Training Course No. 3, Geothermal Resources Council, Albuquerque, NM, 23 to 24 March 1980.

44. Gillingham, E.I., Manager of Petroleum Products, Jet Research Center, Personal communication.

45. Anon, "Temperature Characteristics of HMX," E.I. DuPont de Nemours \& Co., Explosives Department, Chemical Sales Division, Wilmington 98, Delaware, 1966.

46. Eichelberger, R.J. and M.P. Emerson, "Experimental Verification of the Theory of Jet Formation by Charges with Lined Conical Cavities," Journal of Applied Physics, Vol 23, No. 5, May 1952 .

47. Bell, W.T. and G.A. Auberlinder, "Perforating High-Temperature Wells," Journal of Petroleum Technology, March 1961

\subsection{References for Subsection 3.1}

48. Shyrock, S.H. and D.K. Smith, "Geothermal Cementing - The State of the Art," Proceedings of the International Conference on Geothermal Drilling and Completion Technology, 21 to 23 January 1981.

49. Snyder, R.E., "Geothermal Well Casing Failure Modes," ASME Publication 80-PET-84.

50. Smith, D.K. and S.H. Shyrock, "Geothermal Cementing," presented at Basic Geothermal Drilling and Completion Technology School in Albuquerque, NM, 24 to 26 March 1980. 
51. Snyder, R.E., "How Geothermal Wells are Completed and Produced," World Oil, October 1979.

52. Aguirre, B.D. and F.V. Blanco, "Geothermal Well Maintenance and Repair in Cerro Prieto," Procedures of the International Conference on Geothermal Drilling and Completion, 21 to 23 January 1981 .

53. Carson, C.C. and Y.T. Lin, "Geothermal Well Costs and Their Sensitivities to Changes in Drilling and Completion Operation," Procedures of the International Conference on Geothermal Drilling and Completion, 21 to 23 January 1981.

54. Pye, S., "Drilling, Completing and Maintaining Geothermal Wells in Baca, New Mexico," Procedures of the International Conference on Geothermal Drilling and Completion, 21 to 23 January 1981.

55. Suman, G.O. and R.C. Ellis, World Oils Cementing Handbook, Gulf Publishing Co., 1977.

56. "Casing Centralizers," API Standards 10D, 2nd Ed., API, February 1973.

57. Personal communication with Rudy Callahan, Bakerline.

58. Snyder, R.E., "Geothermal Well Casing Design - A study of Failure Modes and Cementing Limitations," Work performed under Sandia Contract No. 13-2297, May 1979.

59. Letter from R.E. Snyder relating conversation with Rudy Callahan, Bakerline.

60. Metals Handbook, Vol. 1, 8th Ed., American Society of Metals, 1961 .

61. Pettitt, R.A., "Monitoring and Repairing Geothermal Casing Cement: A Case History," Geothermal Research Council, Transactions, Vol. 4, September 1980.

62. Baca Project Data and Reports published by Union Oil Company of California, Santa Rosa, CA, 1976.

63. Wood, E.T., "Primary Cementing Equipment and Critical Development Needs," Report to be published under Sandia Contract No. 46-0173.

64. Republic Steel Catalog of Casing Data.

65. Standard Handbook for Mechanical Engineers, 7th Ed., T. Baumeister, Editor in Chief, McGraw-Hill, 1967. 
66. Nelson, E.B., L.H. Eiles, L.K. Moran, L.B. Spangle, and B.E. Simpson, "Development of Geothermal Well Completion Systems," Geothermal Research Council, Transactions, Vol. 3, September 1979 .

67. Rabinowicz, E., Friction and Wear of Materials, John Wiley \& Sons, 1965.

68. Glass, W.A., "1977 Drilling Methods and Costs at the Geysers," Geothermal Resources Council, Transactions, Vol. 1, May 1977.

69. "Predicting Liner Hanger Capacities," Brown Oil Tools, Inc.

70. Lindsey, H.E., Jr., and S.J. Bateman, "Improved Cementing of Drilling Liners in Deep Wells," World Oil, October 1973, p. 65 .

71. "Deep Gas Well Completion Practices Manual," Texas Iron Works, Inc., Houston, TX, 1972.

72. Kiselman, L.I. and M.L. Kiselman, "Wear of Casing Strings in Deep Wells," Neft. Khoz, Vol. 10, 9-13, October 1970.

73. Personal communication with Rudy Callahan, Bakerline.

74. Personal communication between R.E. Snyder and Texas Iron Works, Inc., March 1981.

75. Bradley, W.B. and J.E. Fontenot, "The Prediction and Control of Casing Wear," Journal of Petroleum Technology, February 1975 .

\subsection{References for Subsection $3: 2$}

76. Moore, D.F., The Friction and Lubrication of Elastomers, Pergamon Press, 1972 .

77. Grosch, K.A., Proc. Phys. Soc., A274, 1963, p. 21.

78. Grosch, K.A. and A. Schallamach, Trans. Inst. Rubber Ind., $41 \mathrm{~T} 80,1965$.

79. 1980-1981 Composite Catalogue of Oil Field Equipment and Services, B\&W Catalogue, P515.

\subsection{Reference for Subsection 3.3}

80. Schlichting, H., Boundary Layer Theory, Fourth Edition, McGraw-Hill Book Company, 1961. 
DO NOT MICEOFILM

DISTRIBUTION :

Tom Anderson

Venture Chemicals

P.0. Box 35845

Houston, Texas 77035

Ed Bingman

She11 Oil Company

Two She11 Plaza

P.O. Box 2099

Houston, Texas 77001

Larry Diamond

Technical Services

Dyna-Dri11

P.O. Box C-19576

Irvine, California 92713

John E. Fontenot

NL Petroleum Services

P.O. Box 60087

Houston, Texas 77205

Dr. Melvin Friedman

Center for Tectonophysics and Dept. of Geology

Texas A\&M University

College Station, Texas 77843

Tom Turner

Phillips Petroleum Company Geothermal Operations

655 East 4500 South

Salt Lake City, Utah 84107

Jim Kingsolver, Manager

Geotherma1 Operations

Smith Too 1

P.0. Box C-19511

Irvine, California 92713

James W. Langford

Security Division

Dresser Industries, Inc.

P.O. Box 24647

Da1las, Texas 75224

B. J. Livesay

129 Liverpool

Cardiff, California 92007

Harvey E. Ma11ory

P.O. Box 54696

Tu1sa, Ok1ahoma 74155

Ed Martin

Superior $0 i 1$

Eastern Division

P.OL Box 51108 OCS

Lafayette, Louisiana 70505

Gene Polk

NL Baroid

6400 Uptown Blvd. N.E., 365W

Albuquerque, New Mexico 87110

Del E. Pyle

Union Geothermal Division

Union Oil Co. of California

Union Oil Center

Los Angeles, California 90017

John C. Rowley

Los Alamos National Labs

Mail Stop 570

Los Alamos, New Mexico 87545

William D. Rumbaugh

Research and Development

Otis

P.O. Box 34380

Da11as, Texas 75234

Dwight Smith

Ha11iburton

Drawer 1431

Duncan, Oklahoma 73533

Tom Warren

Amoco Production Company

Research Center

P.O. Box 591

Tulsa, Oklahoma 74102 


\section{跑}

$\lambda$

U.

DISTRIBUTION (cont.)

U.S. Department of Energy (6)

Geothermal Hydropower

Technologies Division

Forrestal Bldg., CE 324

1000 Independence Ave. S.W.

Washington, D.C. 20585

Attn: J. Bresee

D. Clements

R. Toms

D. A11en

G. E11 is

U.S. Department of Energy

Geopressure Projects office

Suite 8620 , Federal B1dg.

515 Rusk Street

Houston, Texas 77002

Attn: F. L. Goldsberry

3141

3151

L. J. Erickson

(5)

$3154-3$

W. L. Garner

9000

C. Dalin (25) for DOE/TIC

9700

G. A. Fowler

9730

E. H. Beckner

9740

W. D. Weart

9741

9741

9743

9746

9747

9750

9760

9770

9780

8214

R. K. Traeger

J. R. Kelsey

C. C. Carson

H. C. Hardee

B. Granoff

P. J. Hommert

V. L. Dugan

R. W. Lynch

G. E. Brandvold

R. M. Jefferson

M. A. Pound 